David Leonardo Nascimento de Figueiredo Amorim

\title{
FORMULAÇÕES HÍBRIDAS E MISTAS PARA O MÉTODO DOS ELEMENTOS FINITOS GENERALIZADOS: APLICAÇÃO À MECÂNICA DO DANO
}

\begin{abstract}
Dissertação apresentada à Escola de Engenharia de São Carlos, Universidade de São Paulo, como parte dos requisitos para obtenção do título de mestre em Ciências, Engenharia de Estruturas.
\end{abstract}

Orientador: Prof. Tit. Sergio Persival Baroncini Proença

\section{VERSÃO CORRIGIDA}

A versão original encontra-se na Escola de Engenharia de São Carlos

São Carlos, 
Ficha catalográfica preparada pela Seção de Tratamento

da Informação do Serviço de Biblioteca - EESC/USP

Amorim, David Leonardo Nascimento de Figueiredo.

Formulações híbridas e mistas para o método dos

A524f elementos finitos generalizados: aplicação à mecânica do dano. / David Leonardo Nascimento Figueiredo Amorim ; orientador Sergio Persival Baroncini Proença. São Carlos, 2012.

Dissertação (Mestrado - Programa de Pós-Graduação em Engenharia de Estruturas)- - Escola de Engenharia de São Carlos da Universidade de São Paulo, 2012.

1. Formulações não convencionais em elementos finitos. 2. Enriquecimento nodal. 3. Mecânica do dano. I. Título. 


\section{FOLHA DE JULGAMENTO}

Candidato: Engenheiro DAVID LEONARDO NASCIMENTO DE FIGUEIREDO AMORIM.

Título da dissertação: "Formulações híbridas e mistas para o método dos elementos finitos generalizados: aplicação à mecânica do dano".

Data da defesa: $16 / 03 / 2012$

\section{Comissão Julgadora:}

Prof. Titular Sergio Persival Baroncini Proença (Orientador) APROVADO (Escola de Engenharia de São Carlos/EESC)

Prof. Titular José Luiz Antunes de Oliveira e Souza (Universidade Estadual de Campinas/UNICAMP)

Prof. Dr. Ricardo Carrazedo

(Escola de Engenharia de São Carlos/EESC)
Resultado:
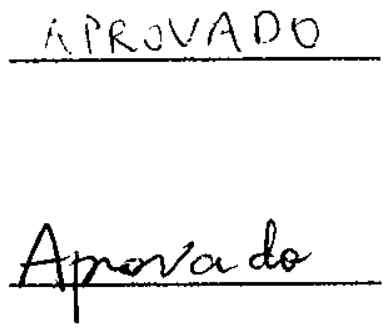

Coordenador do Programa de Pós-Graduação em Engenharia Civil (Engenharia de Estruturas):

Profa. Associada Ana Lucia Homce de Cresce El Debs

Presidente da Comissão de Pós-Graduação:

Prof. Associado Paulo Cesar Lima Segantine 

Dedico este trabalho à minha avó, Neide, à minha mãe, Neide, ao meu irmão, Alfredo, e ao meu tio, João Pedro. 



\section{AGRADECIMENTOS}

Agradeço primeiramente a Deus, por tornar tudo possível em minha vida.

Agradeço à minha família, pelo apoio incondicional e todo carinho e afeto em mim depositados. Minha família é tudo o que tenho, sem ela eu não conseguiria.

Neste parágrafo não quero agradecer, mas sim homenagear o maior guerreiro que conheço: meu irmão Alfredo. De setembro de 2010 a abril de 2011 você lutou por sua vida e todos nós sofremos com você. Eu te admiro e te amo muito meu irmão!

Agradeço aos amigos de infância do conjunto Ipaseal, por sempre me receberem de braços abertos quando vou para Maceió e, também, por todas as nossas idas ao Trapichão nos dias de jogo do CSA, fazendo com que eu matasse a saudade do mais querido, mesmo vivendo longe de casa.

Agradeço aos amigos e professores da UFAL que sempre mantiveram contato, me apoiaram e incentivaram sempre que precisei.

Agradeço aos grandes amigos que também vivem ou viveram em São Carlos. Começando pela "comunidade alagoana", que sempre fizeram com que a saudade de casa fosse a menor possível, sempre reunindo os alagoanos e "agregados" aos domingos para almoços que só saiam depois das 17:00, dentre tantas outras ocasiões. Adiciono aos amigos alagoanos aquelas amizades que fiz em outras universidades e nos outros departamentos da EESC, em especial aos da hidráulica, que conviviam comigo nos rachas de meio de semana, nos churrascos na casa de alguém, onde sempre se tem histórias pra contar, enfim, aqueles que simplificavam a vida em São Carlos. E por fim, agradeço aos amigos do departamento de estruturas pela convivência do dia a dia, principalmente nos cafezinhos, pelos vários momentos de descontração vividos, quando nos reuníamos onde quer que fosse, e também pelas nem sempre disputadas partidas de buraco.

Agradeço também aos funcionários e professores do departamento de estruturas, que se dispuseram a ajudar em todas as situações e sempre com um sorriso no rosto. Dentre estes, agradeço em especial ao Prof. Sergio Proença pela atenção, paciência, dedicação e boa vontade nos momentos que precisei da sua orientação, que não foram poucos.

Agradeço ao CNPq pela bolsa de mestrado concedida, à Universidade de São Paulo Escola de Engenharia de São Carlos pela acolhida e à Universidade Federal de Alagoas pela minha formação. 

"Rapadura é doce, mas não é mole não." (dito popular nordestino) 



\section{RESUMO}

AMORIM, D. L. N. F. (2012). Formulações híbridas e mistas para o método dos elementos finitos generalizados: aplicação à mecânica do dano. Dissertação (Mestrado) - Escola de Engenharia de São Carlos, Universidade de São Paulo, São Carlos.

A formulação convencional do Método dos Elementos Finitos (MEF) baseada em aproximação do campo de deslocamentos apresenta limitações conhecidas como, por exemplo, em problemas que envolvam regiões com singularidade e problemas de elasticidade quase incompressível. Quando a utilização do MEF convencional inclui a análise de propagação de fissuras ou de dano evolutivo, faz-se recurso a mudança contínua da rede de elementos finitos, tornando as simulações onerosas em termos computacionais. Assim, justifica-se a procura por metodologias que combinem boa capacidade de aproximação com baixo custo computacional. Neste trabalho as formulações estudadas são dos tipos híbridomista e puramente mista, ambas combinadas com técnicas de enriquecimento das soluções proporcionadas pelo Método dos Elementos Finitos Generalizados (MEFG). A metodologia numérica resultante é aplicada às análises de sólidos em regime de ruptura modelados pela Mecânica do Dano. Vale ressaltar que a aproximação do campo de tensões, dada por estas formulações não convencionais, pode permitir melhor estimativa da danificação da estrutura, frente ao MEF convencional. Isto se dá porque os critérios de danificação empregados são quase sempre baseados na estimativa do campo de tensões atuantes. Nas análises numéricas, aplica-se uma estratégia incremental-iterativa convencional e propõe-se uma estratégia simplificada não iterativa, que permite uma estimativa do dano utilizando-se os resultados de uma análise linear. O trabalho é finalizado com a comparação entre resultados de simulações desenvolvidas mediante as estratégias simplificada e não linear completa, comprovando-se que o modelo simplificado pode ser empregado nas situações de dano localizado em pequenas regiões do domínio.

Palavras-chave: Formulações não convencionais em elementos finitos, enriquecimento nodal, Mecânica do Dano. 



\begin{abstract}
AMORIM, D. L. N. F. (2012). Hybrid and mixed formulations to the generalized finite element method: application to damage mechanics. MS.c. Thesis - São Carlos School of Engineering, University of São Paulo, São Carlos.
\end{abstract}

The conventional Finite Element Method (FEM) based on displacement field approximation presents limitations e. g. problems involving regions with singularity and problems of quasi-incompressible elasticity. When the conventional FEM utilization includes the crack growth analysis or damage evolution is made resort of the continuous change of finite element mesh, rendering costly simulations in computational terms. Thus, it is justified the search of methodologies which combine good approximation capacity with low computational cost. In this research the studied formulations are of hybrid-mixed and purely mixed types, both combined with enrichment techniques of solutions offered by the Generalized Finite Element Method (GFEM). The numerical resultant methodology is applied to solid analyses in rupture regime modeled using Damage Mechanics. It is noteworthy that the approximation of stress field, given with these nonconventional formulations, may allow better damage estimative of structure, compared to conventional FEM. This is because the employed damage criteria are almost always based on active stress field estimative. In the numerical analyses it is applied a conventional incremental-iterative strategy and it is proposed a non-iterative simplified strategy which allows damage estimative using the results of a linear analysis. The research is finalized with the comparison between results of developed simulations through the simplified and nonlinear strategies, proving that the simplified model may be employed in situations where the damage is located in small regions in domain.

Keywords: Nonconventional formulations in finite elements, nodal enrichment, Damage Mechanics. 



\section{LISTA DE FIGURAS}

Figura 2.1 - Corpo elástico submetido a forças de volume e de superfície, e a representação das partes complementares do contorno.

Figura 3.1 - Elemento de volume $\boldsymbol{\Omega}$ e contornos $\boldsymbol{\Gamma} \boldsymbol{t}, \boldsymbol{\Gamma} \boldsymbol{u}$ e $\boldsymbol{\Gamma} \boldsymbol{i}$. Fonte: Góis (2009). 13

Figura 3.2 - Dois elementos de volume $\boldsymbol{\Omega}$ e contornos $\boldsymbol{\Gamma} \boldsymbol{t}, \boldsymbol{\Gamma} \boldsymbol{u}$ e $\boldsymbol{\Gamma} \boldsymbol{i}$. Fonte: Góis (2009)....... 16 Figura 3.3 - Dois elementos de volume $\boldsymbol{\Omega}$ e contornos $\boldsymbol{\Gamma} \boldsymbol{t}, \boldsymbol{\Gamma} \boldsymbol{u}$ e $\boldsymbol{\Gamma} \boldsymbol{i}$ - continuidade e reciprocidade em forma fraca. Fonte: Góis (2009).

Figura 3.4 - Dois elementos mistos.

Figura 4.1 - Conjunto de nuvens cobrindo um domínio $\Omega$. Fonte: Proença (2010).

Figura 4.2 - Nuvens no MEFG. Fonte: Proença (2010).

Figura 4.3 - Procedimento de enriquecimento sobre um ponto: resultado (inferior) da multiplicação entre a função de forma (superior) com uma função enriquecedora (centro) polinomial (a) e não polinomial (b). Fonte: Duarte e Kim (2008).

Figura 5.1 - Elemento de volume representativo. Fonte: Proença (2008). .37

Figura 5.2 - Análise da função $\psi i n \xi$ para diferentes valores de $n$. 49

Figura 5.3 - Análise da função $\psi i n \xi$ para diferentes valores de $n$ (detalhe). 50

Figura 5.4 - Análise da derivada parcial da função $\psi$ em relação a $\xi . \chi=\psi, \xi$. .50

Figura 5.5 - Resposta de $\chi-\xi$ para diferentes valores de $n$.

Figura 5.6 - Comportamento unidimensional do modelo constitutivo para diferentes valores de $n$.

Figura 6.1 - Geometria, carregamento e vinculação de chapa discreta com dois elementos finitos. 55

Figura 6.2 - Resultados para o teste de distorção (distorção - deslocamento na extremidade livre). .56

Figura 6.3 - Chapa tracionada...... .56

Figura 6.4 - Modelo de análise. .57

Figura 6.5 - Verificação de convergência da FHMT. .... 59

Figura 6.6 - Verificação de convergência da $\operatorname{FM}(u-\sigma)$. 59

Figura 6.7 - Comparação entre a FHMT e a solução de referência. 60

Figura 6.8 - Comparação entre a $\operatorname{FM}(\mathrm{u}-\sigma)$ e a solução de referência. 61

Figura 6.9 - Chapa tracionada com fissura discreta. 61

Figura 6.10 - Modelo matemático do problema. 
Figura 6.11 - Resultado de $\sigma x$ da solução de referência para toda a chapa (esquerda) e detalhe da região da ponta da fissura (direita).

Figura 6.12 - Resultado de $\sigma y$ da solução de referência para toda a chapa (esquerda) e detalhe da região da ponta da fissura (direita).

Figura 6.13 - Resultado de $\tau x y$ da solução de referência para toda a chapa (esquerda) e detalhe da região da ponta da fissura (direita). 63

Figura 6.14 - Dano calculado utilizando-se a solução de referência. 64

Figura 6.15 - Discretização adotada para simulação com o MEFG. 64

Figura 6.16 - Resultado de $\sigma x$ do MEFG para toda a chapa (esquerda) e detalhe da região da ponta da fissura (direita).

Figura 6.17 - Resultado de $\sigma y$ do MEFG para toda a chapa (esquerda) e detalhe da região da ponta da fissura (direita).

Figura 6.18 - Resultado de $\tau x y$ do MEFG para toda a chapa (esquerda) e detalhe da região da ponta da fissura (direita). 66

Figura 6.19 - Dano calculado para a solução do MEFG. 66

Figura 6.20 - Discretizações adotadas para simulação com a FHMT (esquerda) e FM(u- $\sigma)$ (direita).

Figura 6.21 - Resultado de $\sigma x$ da FHMT para toda a chapa (esquerda) e detalhe da região da ponta da fissura (direita).

Figura 6.22 - Resultado de $\sigma y$ da FHMT para toda a chapa (esquerda) e detalhe da região da ponta da fissura (direita). 68

Figura 6.23 - Resultado de $\tau x y$ da FHMT para toda a chapa (esquerda) e detalhe da região da ponta da fissura (direita). 68

Figura 6.24 - Dano calculado utilizando-se a FHMT. 68 Figura 6.25 - Resultado de $\sigma x$ da FM(u- $\sigma$ ) para toda a chapa (esquerda) e detalhe da região da ponta da fissura (direita).

Figura 6.26 - Resultado de $\sigma y$ da $\operatorname{FM}(\mathrm{u}-\sigma)$ para toda a chapa (esquerda) e detalhe da região da ponta da fissura (direita).

Figura 6.27 - Resultado de $\tau x y$ da $\operatorname{FM}(\mathrm{u}-\sigma)$ para toda a chapa (esquerda) e detalhe da região da ponta da fissura (direita). 70

Figura 6.28 - Dano calculado utilizando-se a FM(u- $\sigma)$...... 70

Figura 6.29 - Teste de tração numa chapa...... 71

Figura 6.30 - Comparação das formulações com a solução analítica. 71 
Figura 6.31 - Influência do coeficiente de Poisson na resposta não linear da FHMT em EPD.

Figura 6.32 - Influência do coeficiente de Poisson na resposta não linear da FM(u- $\sigma)$ em

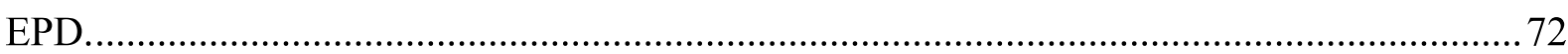

Figura 6.33 - Confronto das respostas de dano para a FHMT........................................... 73

Figura 6.34 - Confronto das respostas de dano para a FM(u- $\sigma)$. ........................................... 74

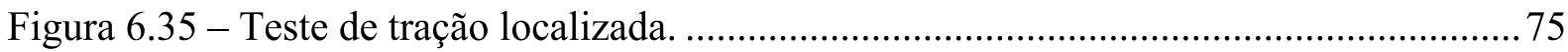

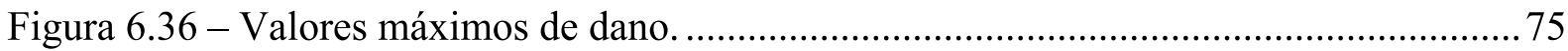

Figura 6.37 - Distribuição do dano para (a) $p=5,50 \mathrm{MPa}$, (b) $p=5,60 \mathrm{MPa}$, (c) $p=$

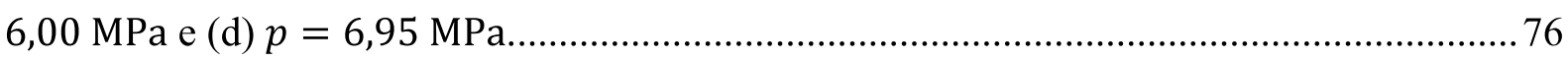

Figura 6.38 - Teste de Hassanzadeh. Fonte: Silva (2006). ................................................. 77

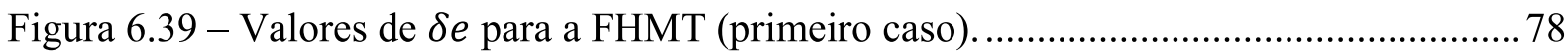

Figura 6.40 - Comparação da distribuição do dano nos pontos de controle (primeiro caso). 79 Figura 6.41 - Comparativo dos valores de dano máximo para o Teste de Hassanzadeh

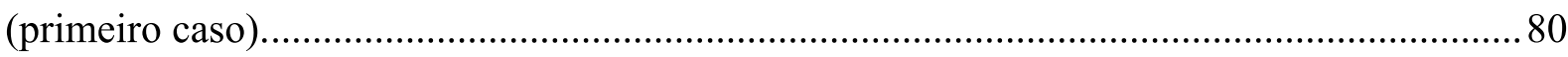

Figura 6.42 - Resposta de ambos os modelos de dano para a carga máxima $R=4756$ N.... 80

Figura 6.43 - Valores de $\delta e$ para a FHMT (segundo caso). .................................................. 81

Figura 6.44 - Comparativo dos valores de dano máximo para o Teste de Hassanzadeh (segundo caso).

Figura 6.45 - Comparação da distribuição do dano nos pontos de controle A, B e C (segundo caso)

Figura 6.46 - Comparação distribuição do dano nos pontos de controle D, E e F (segundo caso). 83

Figura 6.47 - Comparação distribuição do dano nos pontos de controle $\mathrm{G} \mathrm{e} \mathrm{H}$, e para a carga máxima (segundo caso). 84

Figura A.1 - Elemento quadrilateral de quatro nós. Fonte: Góis (2009). 93 Figura A.2 - Elemento finito linear com sistema local de referência com origem no seu centro. Fonte: Góis (2009). .95

Figura A.3 - Elemento quadrilateral de quatro nós. 103 



\section{LISTA DE TABELAS}

Tabela 5.1 - Variáveis de estado e associadas. Fonte: Lemaitre e Desmorat (2005)...............39

Tabela 6.1 - Verificação da estabilidade numérica do modelo para a FHMT. ........................58

Tabela 6.2 - Verificação da estabilidade numérica do modelo para a $\operatorname{FM}(\mathrm{u}-\sigma)$. .....................58 



\section{LISTA DE ABREVIATURAS E SIGLAS}

EPD - Estado Plano de Deformações

EPT - Estado Plano de Tensões

EVR - Elemento de Volume Representativo

FHMD - Formulação Híbrido-Mista de Deslocamento

FHMT - Formulação Híbrido-Mista de Tensão

FM(u- $\sigma)-$ Forma Mista $u-\sigma$

$h p$ - Processos $h$-adaptativo e $p$-adaptativo do Método dos Elementos Finitos

MEF - Método dos Elementos Finitos

MEFG - Método dos Elementos Finitos Generalizados

MMQM - Método dos Mínimos Quadrados Móveis

PU - Partição da Unidade

PVC - Problema de Valor de Contorno 



\section{LISTA DE SÍMBOLOS}

$\boldsymbol{A} \quad$ Matriz de compatibilidade generalizada da FM(u- $\sigma)$

$\boldsymbol{A}^{+}, \boldsymbol{A}^{-} \quad$ Matriz de compatibilidade generalizada do elemento misto

$\boldsymbol{A}_{\boldsymbol{\Omega}} \quad$ Matriz de compatibilidade generalizada de domínio da FHMT

$\boldsymbol{A}_{\boldsymbol{\Gamma}_{\boldsymbol{i}}} \quad$ Matriz de compatibilidade generalizada de contorno interno da FHMT

$\boldsymbol{A}_{\boldsymbol{\Gamma}_{i}}^{+}, \boldsymbol{A}_{\boldsymbol{\Gamma}_{\boldsymbol{i}}}^{-} \quad$ Matriz de compatibilidade generalizada de contorno interno do elemento híbrido-misto

$\boldsymbol{A}_{\boldsymbol{\Gamma}_{\boldsymbol{t}}} \quad$ Matriz de compatibilidade generalizada de contorno da FHMT

$\boldsymbol{\alpha} \quad$ Encruamento cinemático

$\alpha \quad$ Partição da unidade ou nuvem do MEFG

$\langle\boldsymbol{B}(\cdot)\rangle \quad$ Matriz Booleana

b Vetor de forças volúmicas

$\boldsymbol{b}^{+}, \boldsymbol{b}^{-} \quad$ Vetor de forças volúmicas do elemento

$\bar{b} \quad$ Vetor de forças volúmicas conhecidas

$b_{\alpha i} \quad$ Parâmetros nodais adicionais introduzidos pelo enriquecimento

$\Gamma \quad$ Contorno

$\Gamma_{\boldsymbol{i}} \quad$ Contorno interno ou entre elementos

$\boldsymbol{\Gamma}_{\boldsymbol{i}}^{+}, \boldsymbol{\Gamma}_{\boldsymbol{i}}^{-} \quad$ Contorno interno do elemento

$\Gamma_{\boldsymbol{t}} \quad$ Contorno estático ou de Neumann

$\boldsymbol{\Gamma}_{\boldsymbol{u}} \quad$ Contorno cinemático ou de Dirichlet

$\gamma \quad$ Escalar positivo do modelo não linear

$\gamma_{x y} \quad$ Distorção angular no plano xy

$\boldsymbol{C}^{+}, \boldsymbol{C}^{-} \quad$ Integral no contorno interno do produto entre as funções de aproximação das forças de superfície entre elementos da $\operatorname{FM}(u-\sigma)$ e as funções de aproximação dos campos de deslocamento de domínio do elemento

$\boldsymbol{C}_{\boldsymbol{\Gamma}_{\boldsymbol{i}}}^{+}, \boldsymbol{C}_{\boldsymbol{\Gamma}_{\boldsymbol{i}}}^{-} \quad$ Integral no contorno interno do produto entre as funções de aproximação dos campos de deslocamento de contorno interno do elemento e as funções de aproximação das forças de superfície no contorno interno do elemento

C Parâmetro do material relacionado à parte linear do encruamento cinemático

$\bar{C} \quad$ Constante de normalização

c Parâmetro do material para o modelo não linear 
D Matriz constitutiva de rigidez

D Dano anisotrópico

D Dano isotrópico

$D_{c} \quad$ Dano crítico

$D_{0} \quad$ Dano inicial

$\delta \quad$ Tamanho de uma fissura discreta

$\delta_{i j} \quad$ Delta de Dirac

Sm Funções ponderadoras para a relação que contém as condições de compatibilidade e constitutiva

$\delta \mathbf{m}_{\boldsymbol{\Omega}} \quad$ Parâmetros das funções ponderadoras para a relação que contém as condições de compatibilidade e constitutiva

$\delta \mathbf{p} \quad$ Funções ponderadoras da condição de equilíbrio no domínio para a FHMT e FM(u- $\sigma)$ e das condições de contorno de Neumman da FM(u- $\sigma)$

$\delta \mathbf{p}_{\Omega} \quad$ Parâmetros das funções ponderadoras da condição de equilíbrio no domínio para a FHMT e FM(u- $\sigma)$ e das condições de contorno de Neumman da FM(u$\sigma)$

$\delta \boldsymbol{p}_{\boldsymbol{\Gamma}_{\boldsymbol{i}}} \quad$ Vetor dos parâmetros de forças de superfície virtuais no contorno interno

$\delta p \quad$ Parâmetros nodais associados às forças virtuais

$\delta \boldsymbol{q}_{\boldsymbol{\Omega}} \quad$ Vetor dos parâmetros de deslocamento virtual de domínio

$\delta \boldsymbol{q}_{\boldsymbol{\Gamma}_{\boldsymbol{i}}} \quad$ Vetor dos parâmetros de deslocamento virtual de contorno interno

$\delta \mathbf{r} \quad$ Funções ponderadoras das condições de contorno de Neumman da FHMT

$\delta \mathbf{r}_{\mathbf{\Gamma}_{\boldsymbol{t}}} \quad$ Parâmetros das funções ponderadoras das condições de contorno de Neumman da FHMT

$\delta S \quad$ Área bruta do EVR

$\delta S_{D} \quad$ Área de cavidades do EVR

$\delta \tilde{S} \quad$ Área efetiva do EVR

$\delta \boldsymbol{t} \quad$ Forças virtuais

$\delta \boldsymbol{u} \quad$ Vetor de deslocamentos virtuais

$\delta \boldsymbol{u}_{\boldsymbol{\Gamma}_{\boldsymbol{i}}} \quad$ Vetor de deslocamentos virtuais de contorno interno

E Módulo de elasticidade

$\bar{e} \quad$ Erro relativo

$\boldsymbol{e}_{\boldsymbol{\Gamma}_{\boldsymbol{u}}} \quad$ Vetor de deformações generalizadas 
Deformação

$\varepsilon_{x} \quad$ Deformação na direção $\mathrm{x}$

$\varepsilon_{y} \quad$ Deformação na direção y

$\varepsilon$

Representação matricial do tensor de deformações

$\varepsilon^{e}$

Representação matricial do tensor de deformações elásticas

$\varepsilon^{p}$

Representação matricial do tensor de deformações plásticas

$\boldsymbol{\varepsilon}_{\mathbf{0}} \quad$ Estado de deformação inicial

$v \quad$ Coeficiente de Poisson

$\boldsymbol{F} \quad$ Matriz de flexibilidade generalizada

F Potencial de dissipação

$F_{N} \quad$ Família de funções de forma do MEFG considerando todas as nuvens

F Matriz de flexibilidade generalizada com dano

$F_{D} \quad$ Potencial de dano

$F_{X} \quad$ Potencial de encruamento cinemático não linear

$f \quad$ Matriz constitutiva de flexibilidade

$\tilde{\boldsymbol{f}} \quad$ Matriz constitutiva de flexibilidade com dano

$f \quad$ Critério de plastificação

$G_{c} \quad$ Energia crítica de fratura

$H(\cdot) \quad$ Função Heaviside

$I(\alpha) \quad$ Contador para o número de funções de enriquecimento

K Matriz secante ao sistema governativo

$k \quad$ Parâmetro do material para o modelo não linear

L Matriz de derivadas parciais

$L_{i(\alpha)} \quad$ Base de funções de enriquecimento adotadas

$\Lambda(r) \quad$ Função que denota a lei de encruamento

$\dot{\lambda} \quad$ Multiplicador plástico

$\mathbf{M}_{\boldsymbol{\Omega}} \quad$ Funções de aproximação das funções ponderadoras para a relação que contém as condições de compatibilidade e constitutiva

N Matriz dos cossenos diretores

$\mathbf{N}_{+}, \mathbf{N}_{-} \quad$ Matriz dos cossenos diretores do elemento

$N \quad$ Número de nuvens

$N_{D} \quad$ Número de pontos com danificação 


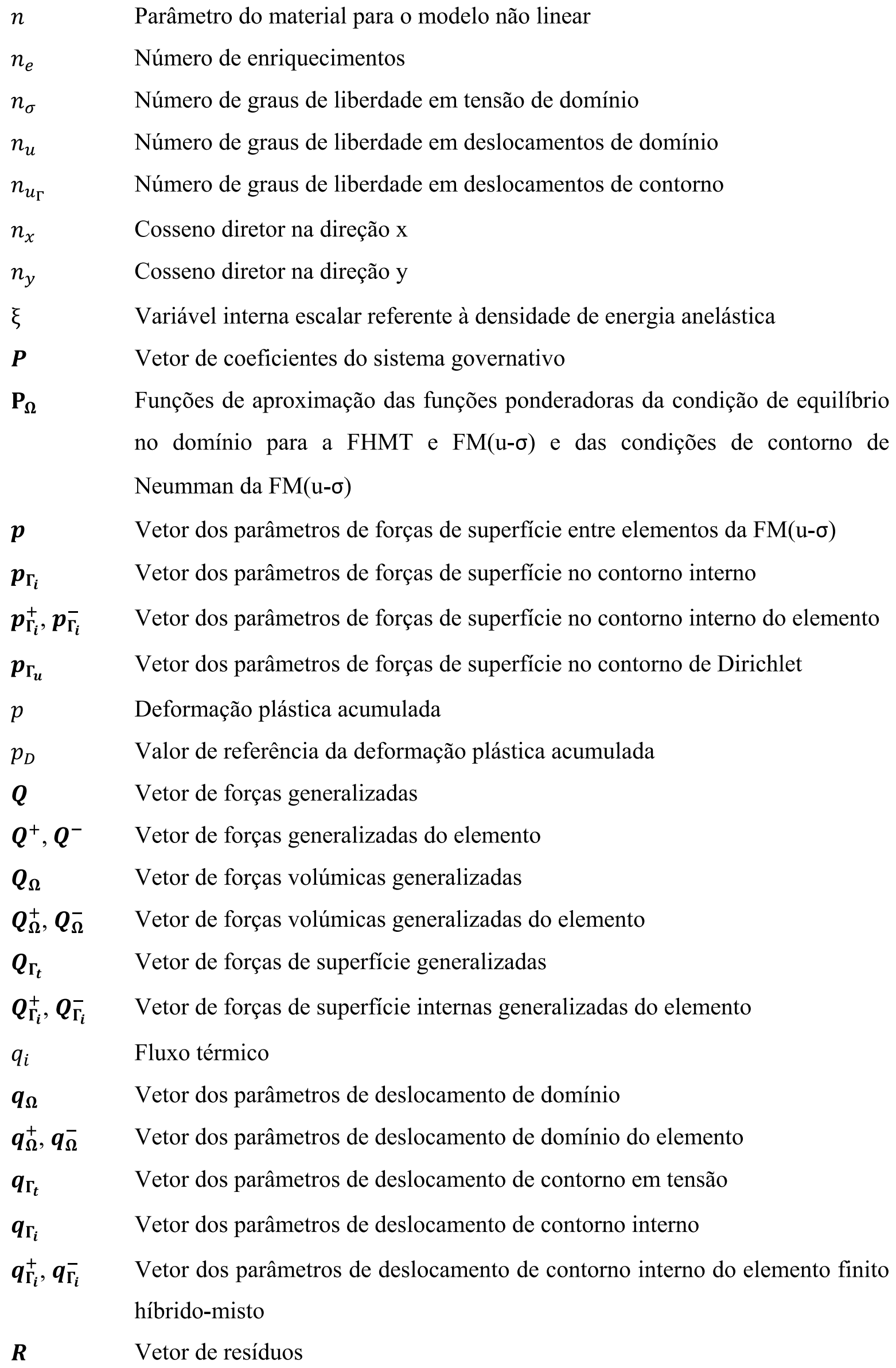




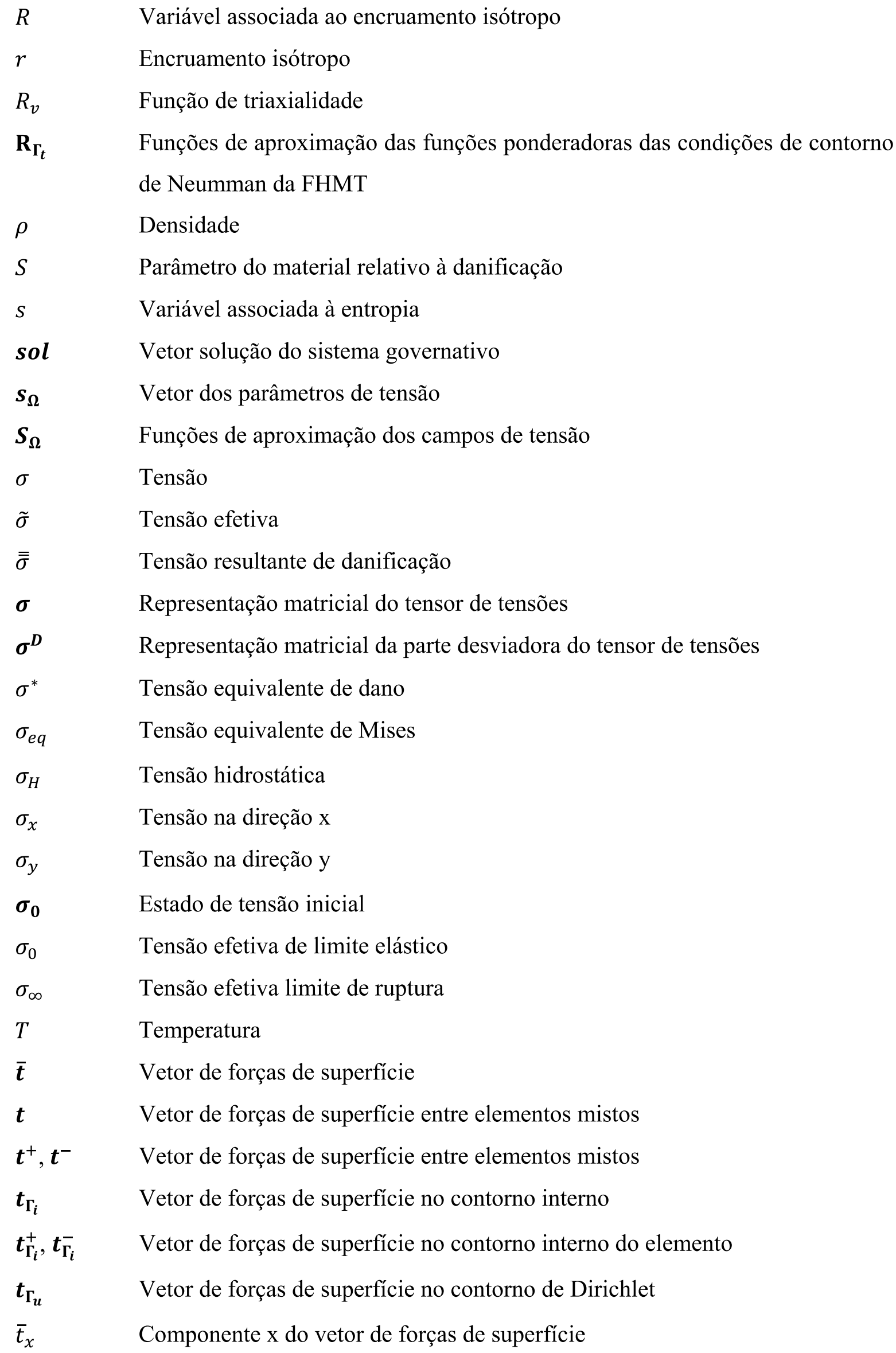




\begin{tabular}{|c|c|}
\hline $\bar{t}_{y}$ & Componente y do vetor de forças de superfície \\
\hline$\tau_{x y}$ & Tensão de cisalhamento no plano xy \\
\hline$U$ & Energia de deformação \\
\hline $\boldsymbol{U}_{\boldsymbol{\Omega}}$ & Funções de aproximação dos campos de deslocamento de domínio \\
\hline $\boldsymbol{U}_{\boldsymbol{\Omega}}^{+}, \boldsymbol{U}_{\boldsymbol{\Omega}}^{-}$ & $\begin{array}{l}\text { Funções de aproximação dos campos de deslocamento de domínio do } \\
\text { elemento }\end{array}$ \\
\hline $\boldsymbol{U}_{\boldsymbol{\Gamma}_{\boldsymbol{i}}}$ & Funções de aproximação dos campos de deslocamento de contorno interno \\
\hline $\boldsymbol{U}_{\Gamma_{i}}^{+}, \boldsymbol{U}_{\Gamma_{i}}^{-}$ & $\begin{array}{l}\text { Funções de aproximação dos campos de deslocamento de contorno interno do } \\
\text { elemento }\end{array}$ \\
\hline$U_{\Gamma_{t}}$ & $\begin{array}{l}\text { Funções de aproximação dos campos de deslocamento de contorno de } \\
\text { Neumann }\end{array}$ \\
\hline$u$ & Deslocamento \\
\hline$u_{x}$ & Deslocamento na direção x \\
\hline$u_{y}$ & Deslocamento na direção y \\
\hline $\bar{u}$ & Deslocamento prescrito \\
\hline$\tilde{u}$ & Aproximação dos deslocamentos pelo MEFG \\
\hline$u_{j}$ & $\begin{array}{l}\text { Parâmetros associados aos graus de liberdade nodais usuais de elementos } \\
\text { finitos }\end{array}$ \\
\hline $\boldsymbol{u}$ & Vetor de deslocamentos \\
\hline $\boldsymbol{u}^{+}, \boldsymbol{u}^{-}$ & Vetor de deslocamentos do elemento \\
\hline$\overline{\boldsymbol{u}}$ & Vetor de deslocamentos prescritos \\
\hline $\boldsymbol{u}_{\boldsymbol{\Gamma}}$ & Vetor de deslocamentos de contorno \\
\hline $\boldsymbol{u}_{\boldsymbol{\Gamma}_{\boldsymbol{i}}}$ & Vetor de deslocamentos de contorno interno \\
\hline $\boldsymbol{u}_{\Gamma_{i}}^{+}, \boldsymbol{u}_{\Gamma_{i}}^{-}$ & Vetor de deslocamentos de contorno interno do elemento \\
\hline$\varphi_{i}$ & Função de forma \\
\hline$\varphi_{\alpha}$ & Função de aproximação básica de uma nuvem ou PU \\
\hline$\Phi_{j}^{1}$ & Função de aproximação do MEFG que matem as características do MEF \\
\hline$\Phi_{\alpha}^{i}$ & Função de aproximação básica de uma nuvem com enriquecimento \\
\hline$\chi$ & Força termodinâmica \\
\hline$\psi$ & Energia livre específica de Helmholtz \\
\hline$\psi_{e}$ & Parcela elástica da energia livre específica de Helmholtz \\
\hline$\psi_{\text {in }}$ & Densidade de energia anelástica \\
\hline
\end{tabular}


$\psi_{p} \quad$ Parcela plástica da energia livre específica de Helmholtz

$\psi_{T} \quad$ Parcela térmica da energia livre específica de Helmholtz

$\psi^{*} \quad$ Potencial da energia específica livre de Gibbs

$\psi_{e}^{*} \quad$ Parcela elástica do potencial da energia específica livre de Gibbs

$\mathbf{\Omega} \quad$ Domínio

$\omega_{\alpha} \quad$ Nuvem

$\omega_{s} \quad$ Densidade de energia armazenada no encruamento

W Funções de aproximação das forças de superfície entre elementos da FM(u- $\sigma)$

$\boldsymbol{W}_{\boldsymbol{\Gamma}_{\boldsymbol{i}}} \quad$ Funções de aproximação das forças de superfície no contorno interno

$\boldsymbol{W}_{\boldsymbol{\Gamma}_{\boldsymbol{i}}}^{+}, \boldsymbol{W}_{\boldsymbol{\Gamma}_{\boldsymbol{i}}}^{-} \quad$ Funções de aproximação das forças de superfície no contorno interno do elemento

$\boldsymbol{W}_{\boldsymbol{\Gamma}_{\boldsymbol{u}}} \quad$ Funções de aproximação das forças de superfície no contorno de Dirichlet

$\boldsymbol{X} \quad$ Variável associada ao encruamento cinemático

$\boldsymbol{Y} \quad$ Densidade de energia liberada associada ao dano anisotrópico

$Y \quad$ Densidade de energia liberada associada ao dano isotrópico

$Y_{c} \quad$ Valor crítico da densidade de energia liberada

$\beth_{\alpha} \quad$ Conjunto de funções de forma de uma nuvem 



\section{SUMÁRIO}

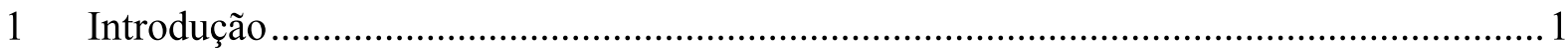

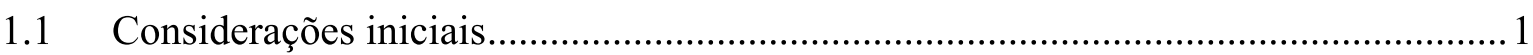

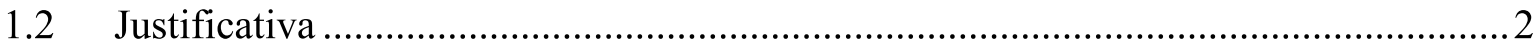

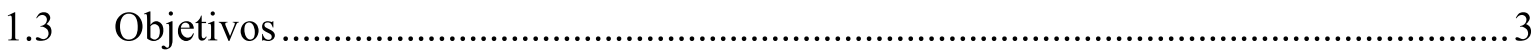

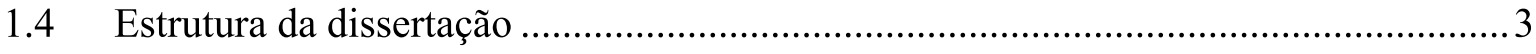

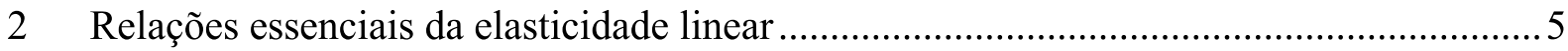

3 Formulações não convencionais em elementos finitos..............................................

3.1 Formulação Híbrido-Mista para elasticidade plana ...............................................

3.2 Forma Mista $\mathrm{u}-\sigma$ para elasticidade plana ...................................................... 21

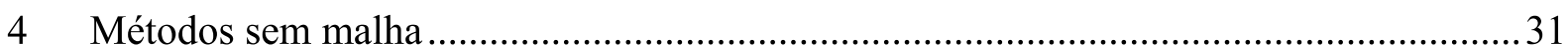

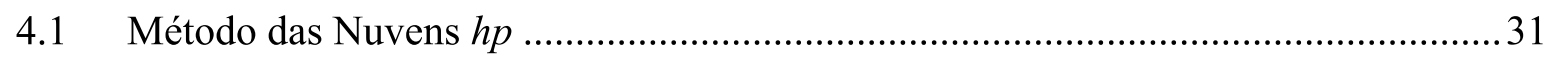

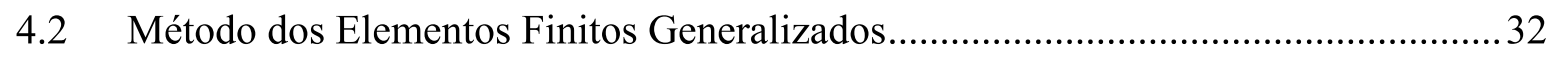

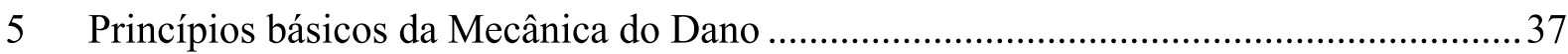

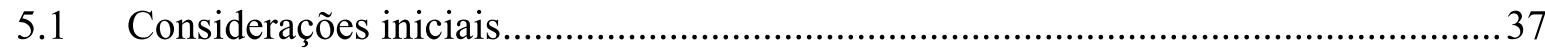

5.2 Elementos da termodinâmica dos processos irreversíveis ........................................38

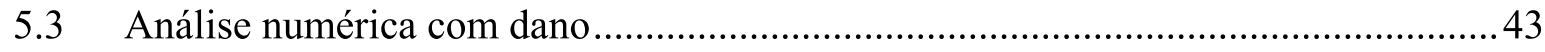

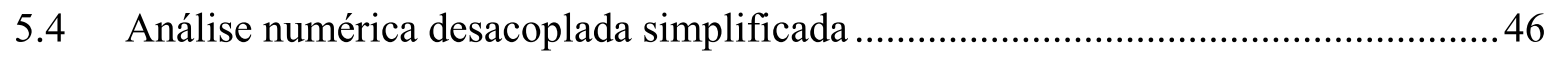

5.5 Análise simplificada elasto-plástica localmente acoplada com dano ......................47

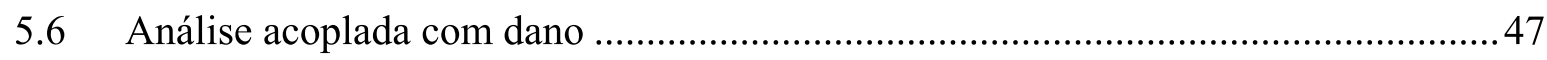

5.6.1 Um modelo de dano isótropo com variável de dano escalar ............................47

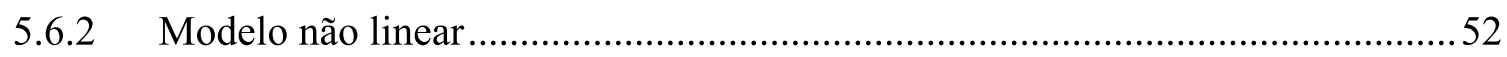

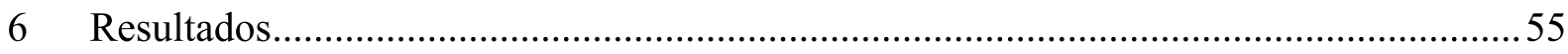

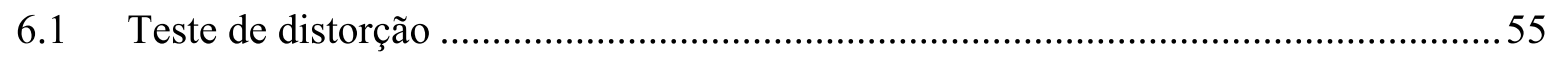

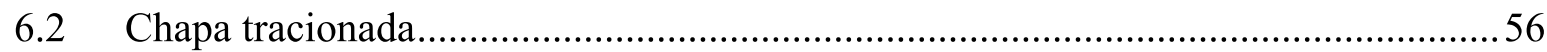

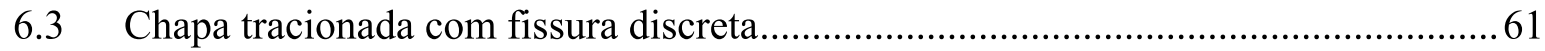

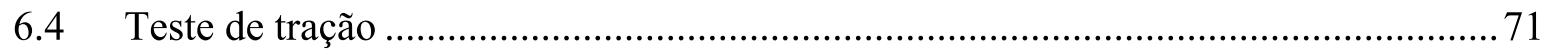




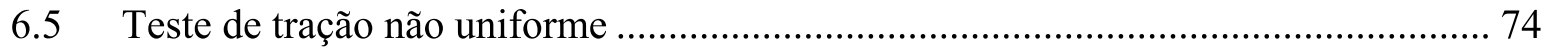

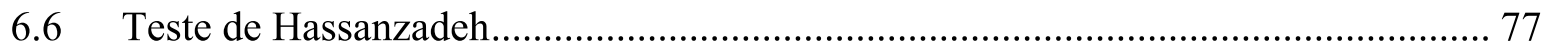

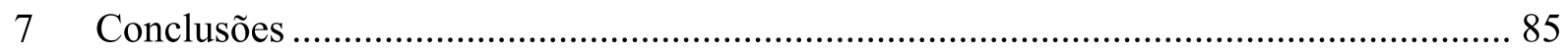

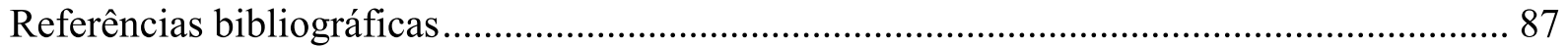

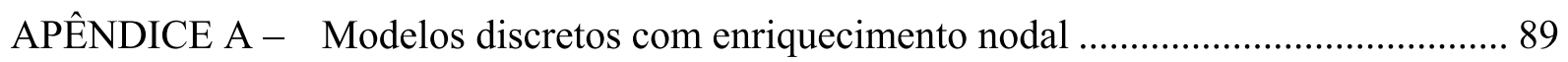

A.1 Modelo discreto da FHMT com enriquecimento nodal ...................................... 89

A.2 Modelo discreto da FM(u- $\sigma)$ com enriquecimento nodal................................... 101

APÊNDICE B - Solução de sistemas: método de perturbação.......................................... 109 


\section{Introdução}

\subsection{Considerações iniciais}

Em qualquer ramo da engenharia é fundamental o entendimento e a consideração do comportamento dos materiais que compõem determinada estrutura. Diversas estruturas, como edifícios, carros, pontes, aviões, navios e próteses substitutivas de ossos humanos, entre outros inúmeros exemplos de várias áreas da engenharia, têm formulação matemática dos seus respectivos materiais baseada em modelos mecânicos-estruturais que procuram se aproximar à realidade.

A modelagem matemática do comportamento de sólidos e estruturas envolve a interação de três grandezas: os deslocamentos $(u)$, as tensões $(\sigma)$ e as deformações $(\varepsilon)$; seguindo uma abordagem conceitual pela Mecânica do Contínuo. O denominado Problema de Valor de Contorno (PVC) reúne restrições sobre os deslocamentos, as deformações e as tensões, formalizadas pelas relações de equilíbrio, de compatibilidade, leis constitutivas e condições de contorno.

Mediante a adoção de hipóteses simplificadoras, soluções analíticas podem ser obtidas somente para um pequeno número de problemas. Já o emprego de métodos numéricos permite a obtenção de soluções aproximadas para toda uma classe de PVC. Estas são obtidas a partir de um modelo discreto concebido com o objetivo de determinar os deslocamentos $(u)$, as tensões $(\sigma)$ e as deformações $(\varepsilon)$ em um número finito de pontos. Dentre os métodos numéricos, o Método dos Elementos Finitos (MEF), em sua formulação em deslocamentos, é um dos mais difundidos para aplicação e resolução de forma aproximada de PVC. Na verdade, a formulação em deslocamentos decorre da forma como é expresso o PVC, sendo independente do MEF. Neste trabalho o MEF é entendido como o resultado da aplicação de uma técnica sistemática para geração de funções interpoladoras em diferentes formas variacionais que o PVC pode ser expresso. As formas ditas variacionais podem estar associadas a um princípio variacional, como o Princípio da Mínima Energia Potencial e o Princípio da Mínima Energia Complementar, ao Princípio dos Trabalhos Virtuais, ou mesmo à ponderação da forma forte do modelo estudado.

A formulação em deslocamentos do MEF, apesar de bastante utilizada, caracteriza-se pela crescente perda de precisão nas ordens superiores de derivadas da função aproximativa. A solução final do campo de tensão de uma análise linear, por exemplo, pode apresentar baixa 
Formulações Híbridas e Mistas para o Método dos Elementos Finitos Generalizados: aplicação à Mecânica do Dano

precisão, pois depende de derivadas da solução em deslocamentos. Por outro lado, é bastante custoso obter respostas com a formulação clássica para problemas que envolvam regiões com singularidade e problemas de elasticidade quase incompressível, conforme justificam Szabó e Babuška (1991) e Bathe (1996). Além disso, a solução obtida pelo MEF clássico também pode ficar comprometida quando se empregam elementos com formas distorcidas.

\subsection{Justificativa}

A análise de meios fraturados via elementos finitos tem sido motivo de diversos estudos nas últimas décadas. A formulação clássica do MEF para este tipo de análise pode-se mostrar computacionalmente inviável, pois a depender do problema, como o de propagação de fissuras, há a necessidade de mudanças contínuas da rede de elementos finitos. Assim, o estudo de formulações não convencionais para resolução deste problema tem por objetivo desenvolver metodologias que combinem boa capacidade de aproximação com baixo custo computacional. Neste trabalho são abordadas duas formulações não convencionais, sendo uma do tipo Híbrido-Mista e a outra puramente Mista.

Ambas as formulações têm como principais vantagens: a possibilidade de aproximação independente dos campos em deslocamentos e tensões envolvidas no PVC, e a escolha de diferentes graus de aproximação destes campos. Tais formulações são ditas mistas porque se fundamentam na aproximação direta de dois campos incompatíveis no domínio (tensões e deslocamentos). Já a primeira delas também se caracteriza como híbrida porque no contorno do problema são feitas aproximações para deslocamentos. Tais formulações também podem ser ditas não convencionais por não serem necessariamente baseadas em algum princípio variacional. De fato, todas podem ser obtidas a partir do método dos resíduos ponderados.

Tendo em vista as análises em regime de ruptura (nesse sentido, adianta-se que neste trabalho propõe-se, ainda, uma técnica simplificada para estimativa da danificação do elemento estrutural a partir de uma análise puramente elástica), vale ressaltar que a aproximação do campo de tensões, por estas formulações, pode permitir melhor estimativa da danificação da estrutura, frente ao MEF convencional. Isto se dá porque os critérios de danificação empregados na análise da capacidade resistente do elemento estrutural são quase sempre baseados na estimativa do campo de tensões atuantes.

Por outro lado, o Método dos Elementos Finitos Generalizados (MEFG) pode ser entendido como uma combinação da forma clássica do MEF com técnicas dos métodos sem malha, especificamente a estratégia de enriquecimento da aproximação adotada no Método 
Introdução

das Nuvens $h p$. Neste, a construção do modelo discreto é gerada a partir de pontos nodais dispersos no domínio, sem nenhuma conectividade. A principal característica do Método das Nuvens $h p$ é que o enriquecimento das funções de aproximação é realizado sem a necessidade de introduzir novos pontos nodais ao domínio. O MEFG surgiu também na tentativa de superar alguns entraves dos métodos sem malha, tais como dificuldades na imposição das condições de contorno na forma fraca do problema e no controle do erro da integração numérica.

Neste trabalho, explora-se a técnica de enriquecimento nodal do MEFG nas formulações não convencionais em estudo e desenvolve-se sua aplicação para análises não lineares associadas a processos de danificação. A extensão aos problemas não lineares é limitada a uma metodologia simplificada, de forma também a compatibilizar o conteúdo e complexidade da pesquisa com os prazos regimentais para o mestrado.

\subsection{Objetivos}

Este trabalho tem como objetivo principal contribuir com o estudo de formulações não convencionais do Problema de Valor de Contorno (PVC), combinando-as com técnicas de enriquecimento nodal características do Método dos Elementos Finitos Generalizados (MEFG). As aplicações consideradas incluem problemas com singularidade nos campos de tensão induzidas por descontinuidades geométricas combinada com uma abordagem simplificada de sólidos com defeitos distribuídos, a luz da Mecânica do Dano.

Avaliam-se, inicialmente, os desempenhos das formulações não convencionais, híbrido-mista e puramente mista, avaliados seja em relação ao emprego de diferentes funções enriquecedoras para análises planas, quanto às condições de convergência para uma solução de referência adotada. Posteriormente, avalia-se até que ponto a análise simplificada de dano, proposta neste trabalho, serve como estimativa da perda da capacidade resistente da estrutura, dispensando uma análise não linear completa.

\subsection{Estrutura da dissertação}

Após esta introdução ao trabalho, apresentam-se algumas relações básicas da elasticidade linear (capítulo 2) para suporte às deduções das formulações não convencionais adotadas (capítulo 3).

Em seguida, os métodos sem malha são brevemente conceituados (capítulo 4), enfatizando-se o conceito de funções de enriquecimento nodal do Método das Nuvens $h p$ (item 4.1) e a base teórica do MEFG (item 4.2). 
O capítulo 5 diz respeito à Mecânica do Dano, destacando-se os princípios termodinâmicos de interesse (item 5.2) e uma técnica desacoplada simplificada para estimativa da danificação (item 5.4).

Alguns exemplos numéricos são apresentados no capítulo 6 e as conclusões do trabalho reunidas no capítulo 7. 


\section{Relações essenciais da elasticidade linear}

$\mathrm{O}$ equacionamento aqui apresentado refere-se a problemas de interesse regidos pela Teoria da Elasticidade Linear, dentro dos limites dos pequenos deslocamentos e deformações. Neste trabalho, considera-se o material como um meio elástico com isotropia. Para aprofundar os conceitos na Teoria da Elasticidade Linear, sugerem-se obras como Timoshenko e Goodier (1980), Valliapan (1981) e Xu (1992). Para a apresentação das relações de interesse utilizarse-á a notação matricial e, em alguns momentos, a notação indicial proposta por Einstein.

Considere-se um corpo que pode ser descrito no plano cartesiano ocupando um domínio, ou região, $\boldsymbol{\Omega}$ e limitado por um contorno regular $\boldsymbol{\Gamma}$. Este pode ser dividido em $\boldsymbol{\Gamma}_{\boldsymbol{u}} \mathrm{e}$ $\boldsymbol{\Gamma}_{\boldsymbol{t}}$, que são as regiões do contorno aonde se prescrevem deslocamentos e forças de superfície, respectivamente. Essas duas partes são ditas complementares, pois $\boldsymbol{\Gamma}=\boldsymbol{\Gamma}_{\boldsymbol{u}} U \boldsymbol{\Gamma}_{\boldsymbol{t}}$ e $\boldsymbol{\Gamma}_{\boldsymbol{u}} \cap \boldsymbol{\Gamma}_{\boldsymbol{t}}=$ $\emptyset$. Assim, o corpo pode ser submetido a forças de volume $\boldsymbol{b}$, distribuídas em $\boldsymbol{\Omega}$, e por forças de superfície $\overline{\boldsymbol{t}}$, distribuídas em $\boldsymbol{\Gamma}_{\boldsymbol{t}}$, conforme exemplifica a Figura 2.1. Vale salientar que, matematicamente, os pontos do contorno (superfície) do corpo não pertencem ao seu domínio.

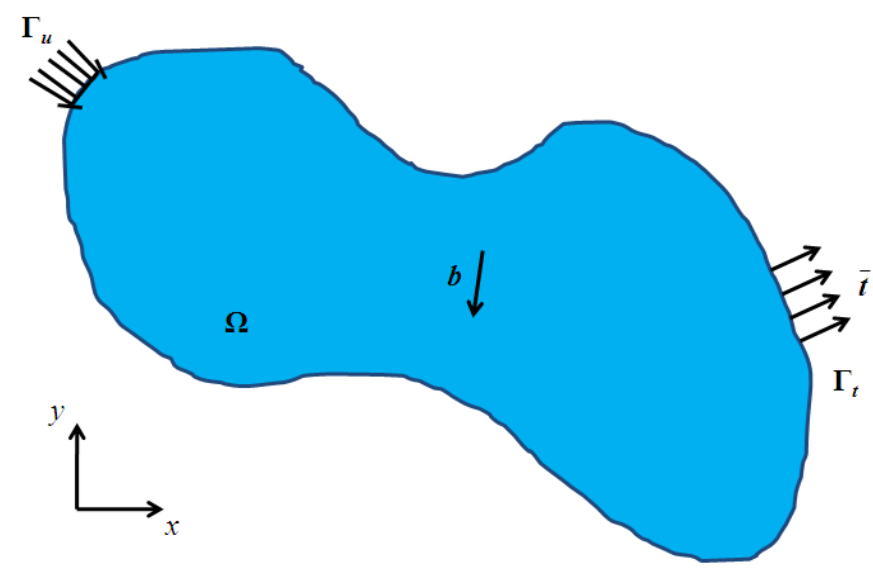

Figura 2.1 - Corpo elástico submetido a forças de volume e de superfície, e a representação das partes complementares do contorno.

O equacionamento do problema elástico linear pode ser dividido em relações constitutivas, de equilíbrio e de compatibilidade. As relações constitutivas, de forma geral, são representadas pelas eq.(2.1) e eq.(2.2):

$\sigma_{i j}=D_{i j k l}\left(\varepsilon_{k l}-\varepsilon_{i j}^{0}\right)+\sigma_{i j}^{0}$ 
$\varepsilon_{i j}=f_{i j k l}\left(\sigma_{k l}-\sigma_{i j}^{0}\right)+\varepsilon_{i j}^{0}$

onde $i, j, k$ e $l$ são valores inteiros entre 1 e 3 . Nas equações observa-se que $D_{i j k l}$ e $f_{i j k l}$ são, respectivamente, as componentes cartesianas dos tensores constitutivos de rigidez e de flexibilidade de quarta ordem; $\sigma_{i j}$ é a componente cartesiana do tensor de tensões de segunda ordem; $\varepsilon_{i j}$ é a componente cartesiana do tensor de deformações de segunda ordem; $\sigma_{i j}^{0}$ e $\varepsilon_{i j}^{0}$ são componentes cartesianas dos tensores de segunda ordem de tensão e deformação iniciais, respectivamente.

As relações de equilíbrio são dadas pela eq.(2.3):

$\sigma_{i j, j}+b_{i}=0$

onde $b_{i}$ é componente cartesiana do vetor das forças de volume. As relações de compatibilidade são expressas conforme a eq.(2.4):

$\varepsilon_{i j}=\frac{1}{2}\left(u_{i, j}+u_{j, i}\right)$

onde $u_{i}$ é componente cartesiana do tensor de primeira ordem dos deslocamentos. Vale ressaltar que a estas equações acrescentam-se as condições de força ou deslocamento prescritas no contorno.

Para o caso particular de estado plano de tensão, as relações constitutivas, de equilíbrio e de compatibilidade são facilmente expressas em notação matricial, valendo-se da hipótese de material elástico linear isótropo, previamente admitida. Isto porque se adota a representação de matriz quadrada aos tensores de quarta ordem, e de matriz com coluna única (vetor) aos tensores de segunda ordem.

Assim, a lei constitutiva passa à representação a seguir:

$\sigma=\mathrm{D}\left(\varepsilon-\varepsilon_{0}\right)+\sigma_{0}$ 
$\varepsilon=f\left(\sigma-\sigma_{0}\right)+\varepsilon_{0}$

sendo:

$$
\boldsymbol{\sigma}=\left\{\begin{array}{c}
\sigma_{x} \\
\sigma_{y} \\
\tau_{x y}
\end{array}\right\}, \quad \boldsymbol{\varepsilon}=\left\{\begin{array}{c}
\varepsilon_{x} \\
\varepsilon_{y} \\
\gamma_{x y}
\end{array}\right\}, \quad \mathbf{D}=\frac{\mathrm{E}}{1-\mathrm{v}^{2}}\left[\begin{array}{ccc}
1 & v & 0 \\
v & 1 & 0 \\
0 & 0 & \frac{1-v}{2}
\end{array}\right], \quad \boldsymbol{f}=\frac{1}{\mathrm{E}}\left[\begin{array}{ccc}
1 & -\mathrm{v} & 0 \\
-\mathrm{v} & 1 & 0 \\
0 & 0 & 2(1+\mathrm{v})
\end{array}\right]
$$

onde E é o módulo de elasticidade e $v$ é o coeficiente de Poisson. Vale lembrar que para o caso particular de Estado Plano de Deformações (EPD), basta substituir:

$$
\bar{v}=\frac{v}{1-v} \quad \bar{E}=\frac{E}{1-v^{2}}
$$

nas relações dadas para o Estado Plano de Tensões (EPT).

As relações de equilíbrio, para a representação bidimensional, passam a ser expressas como abaixo:

$\mathbf{L} \boldsymbol{\sigma}+\boldsymbol{b}=\mathbf{0} \quad \therefore \mathbf{L}=\left[\begin{array}{ccc}\frac{\partial}{\partial x} & 0 & \frac{\partial}{\partial y} \\ 0 & \frac{\partial}{\partial y} & \frac{\partial}{\partial x}\end{array}\right] \quad$, em $\boldsymbol{\Omega}$

sendo $\mathbf{L}$ um operador diferencial de divergência ( $\mathbf{L}^{\mathrm{T}}$ é um operador gradiente) e $\boldsymbol{b}$ é o vetor de forças volúmicas. As relações de compatibilidade são expressas conforme a eq.(2.8):

$\boldsymbol{\varepsilon}-\mathbf{L}^{\mathbf{T}} \boldsymbol{u}=\mathbf{0} \quad$, em $\boldsymbol{\Omega}$

sendo $\boldsymbol{u}=\left\{\begin{array}{l}u_{x} \\ u_{y}\end{array}\right\}$ o vetor de deslocamentos. As condições de contorno na elasticidade plana são:

$\overline{\boldsymbol{u}}-\boldsymbol{u}=\mathbf{0} \quad, \mathrm{em} \boldsymbol{\Gamma}_{\boldsymbol{u}}$ 
$\bar{t}-\mathbf{N} \boldsymbol{\sigma}=\mathbf{0} \quad, \mathrm{em} \boldsymbol{\Gamma}_{\boldsymbol{t}}$

onde $\mathbf{N}=\left[\begin{array}{ccc}n_{x} & 0 & n_{y} \\ 0 & n_{y} & n_{x}\end{array}\right]$ é a matriz construída com as componentes do vetor $n$ normal ao contorno, $\overline{\boldsymbol{u}}$ é o vetor dos deslocamentos impostos em $\boldsymbol{\Gamma}_{\boldsymbol{u}}$ e $\overline{\boldsymbol{t}}=\left\{\begin{array}{l}\bar{t}_{x} \\ \bar{t}_{y}\end{array}\right\}$ é o vetor das forças superficiais aplicadas em $\boldsymbol{\Gamma}_{\boldsymbol{t}}$ 


\section{Formulações não convencionais em elementos finitos}

As formulações em elementos finitos apresentadas neste capítulo são ditas não convencionais porque não se baseiam em princípios variacionais, mas sim no Método dos Resíduos Ponderados.

\subsection{Formulação Híbrido-Mista para elasticidade plana}

Neste item apresenta-se a Formulação Híbrido-Mista para Mecânica dos Sólidos e das Estruturas. De acordo com Góis (2009), admite-se neste desenvolvimento que as condições no contorno de Dirichlet (em deslocamentos) sejam atendidas em forma forte e todas as outras relações impostas em forma fraca, mediante ponderações. Na primeira delas (eq.(3.1)) estão reunidas as condições de compatibilidade e constitutiva, na segunda (eq.(3.2)) a condição de equilíbrio no domínio e na terceira (eq.(3.3)) as condições de contorno de Neumann (em tensões).

$$
\begin{aligned}
& \int_{\Omega} \delta \mathbf{m}^{\mathrm{T}}\left(\mathbf{L}^{\mathrm{T}} u-f \sigma\right) d \Omega=0 \\
& \int_{\Omega} \delta \mathbf{p}^{\mathrm{T}}(\mathbf{L} \sigma+b) d \Omega=0 \\
& \int_{\Gamma_{t}} \delta \mathbf{r}^{\mathrm{T}}(\bar{t}-\mathbf{N} \sigma) d \Gamma=0
\end{aligned}
$$

Aplicando-se o Teorema de Green na primeira integral da eq.(3.1), obtém-se:

$$
\int_{\Omega}(\mathbf{L} \delta \mathbf{m})^{\mathrm{T}} u d \Omega+\int_{\Omega} \delta \mathbf{m}^{\mathrm{T}} \boldsymbol{f} \sigma d \Omega-\int_{\Gamma_{t}}(\mathbf{N} \delta \mathbf{m})^{\mathrm{T}} u_{\Gamma} d \Gamma-\int_{\Gamma_{u}}(\mathbf{N} \delta \mathbf{m})^{\mathrm{T}} \bar{u} d \Gamma=0
$$

$\mathrm{Na}$ relação anterior estão destacados os campos independentes, que justificam a denominação de formulação híbrido-mista: deslocamentos e tensões no domínio $\boldsymbol{\Omega}$, e deslocamentos na parte $\boldsymbol{\Gamma}_{\boldsymbol{t}}$ do contorno. Admite-se, portanto, que as aproximações para deslocamentos no domínio e no contorno são diferentes, pois ambos os campos são 
compatibilizados em forma fraca mediante a relação anterior; e que as tensões e deslocamentos no domínio são incompatíveis. Em termos gerais, nos contornos as grandezas desconhecidas podem ser aproximadas de forma independente das aproximações adotadas no domínio. Como nos contornos $\boldsymbol{\Gamma}_{\boldsymbol{u}}$ e $\boldsymbol{\Gamma}_{\boldsymbol{t}}$ do domínio $\boldsymbol{\Omega}$ são conhecidos $\overline{\boldsymbol{u}}$ e $\overline{\boldsymbol{t}}$, respectivamente, neles são aproximadas, também respectivamente, as grandezas $\boldsymbol{t}$ e $\boldsymbol{u}$.

As aproximações para os campos incógnitos são dadas a seguir, assim como às ponderações adotadas:

$\boldsymbol{\sigma}=S_{\Omega} s_{\Omega}$

$\boldsymbol{u}=\boldsymbol{U}_{\Omega} \boldsymbol{q}_{\Omega}$

$u_{\Gamma}=U_{\Gamma_{t}} q_{\Gamma_{t}}$

$\delta \mathbf{m}=\mathbf{M}_{\Omega} \delta \mathbf{m}_{\Omega}$

$\delta \mathbf{p}=\mathbf{P}_{\Omega} \delta \mathbf{p}_{\Omega}$

$\delta \mathbf{r}=\mathbf{R}_{\mathbf{\Gamma}_{t}} \delta \mathbf{r}_{\Gamma_{t}}$

sendo $\boldsymbol{s}_{\boldsymbol{\Omega}} \mathrm{o}$ vetor dos parâmetros de tensão associados às funções de aproximação dos campos de tensão em $\boldsymbol{S}_{\boldsymbol{\Omega}} ; \boldsymbol{q}_{\boldsymbol{\Omega}}$ o vetor dos parâmetros de deslocamento associados às funções de aproximação dos campos de deslocamento $\boldsymbol{U}_{\boldsymbol{\Omega}}$; e $\boldsymbol{q}_{\boldsymbol{\Gamma}_{\boldsymbol{t}}}$ é o vetor que agrupa os graus de liberdade generalizados associados à matriz das funções de aproximação do campo de deslocamento no contorno $\boldsymbol{U}_{\boldsymbol{\Gamma}_{\boldsymbol{t}}}$.

Substituindo-se essas aproximações no conjunto de relações ponderadas e admitindose que os campos virtuais $\delta \mathbf{m}_{\Omega}, \delta \mathbf{p}_{\Omega}$ e $\delta \mathbf{r}_{\boldsymbol{r}_{\boldsymbol{t}}}$ são compatíveis e homogêneos nas condições de contorno essenciais, obtém-se o seguinte sistema: 
$\left[\begin{array}{ccc}\int_{\Omega} \mathbf{M}_{\Omega}^{\mathrm{T}}\left(f S_{\Omega}\right) d \Omega & \int_{\Omega}\left(\mathbf{L M}_{\Omega}\right)^{\mathrm{T}} U_{\Omega} d \Omega & -\int_{\Gamma_{\mathrm{t}}}\left(\mathrm{NM}_{\Omega}\right)^{\mathrm{T}} U_{\Gamma_{t}} d \Gamma \\ \int_{\Omega} \mathbf{P}_{\Omega}^{\mathrm{T}}\left(\mathbf{L} \mathbf{M}_{\Omega}\right) d \Omega & 0 & 0 \\ -\int_{\Gamma_{\mathrm{t}}} \mathbf{R}_{\Gamma_{t}}^{\mathrm{T}}\left(\mathbf{N M}_{\Omega}\right) d \Gamma & 0 & 0\end{array}\right]\left\{\begin{array}{l}s_{\Omega} \\ q_{\Omega} \\ q_{\Gamma_{t}}\end{array}\right\}=\left\{\begin{array}{c}\boldsymbol{e}_{\Gamma_{u}} \\ -Q_{\Omega} \\ -Q_{\Gamma_{t}}\end{array}\right\}$

onde

$e_{\Gamma_{u}}=\int_{\Gamma_{\mathbf{u}}}\left(\mathrm{NM}_{\Omega}\right)^{\mathrm{T}} \bar{u} d \Gamma$

$Q_{\Omega}=\int_{\Omega} P_{\Omega}^{\mathrm{T}} \bar{b} d \Omega$

$Q_{\Gamma_{t}}=\int_{\Gamma_{t}} \mathbf{R}_{\Gamma_{t}}^{\mathrm{T}} \bar{t} d \Gamma$

Como se observa, o sistema apresentado não é simétrico. Esta condição pode ser alcançada se forem aplicadas restrições tipo Galerkin sobre o conjunto de funções de aproximação:

$$
\mathbf{M}_{\Omega}=S_{\Omega}
$$

$\mathbf{P}_{\Omega}=\boldsymbol{U}_{\boldsymbol{\Omega}}$

$\mathbf{R}_{\Gamma_{t}}=U_{\Gamma_{t}}$

Assim, o sistema resolvente passa a apresentar formato simétrico: 
$\left[\begin{array}{ccc}\boldsymbol{F} & \boldsymbol{A}_{\Omega} & -\boldsymbol{A}_{\boldsymbol{\Gamma}_{t}} \\ \boldsymbol{A}_{\Omega}^{\mathrm{T}} & \mathbf{0} & \mathbf{0} \\ -\boldsymbol{A}_{\boldsymbol{\Gamma}_{t}}^{\mathrm{T}} & \mathbf{0} & \mathbf{0}\end{array}\right]\left\{\begin{array}{l}\boldsymbol{s}_{\Omega} \\ \boldsymbol{q}_{\Omega} \\ \boldsymbol{q}_{\Gamma_{t}}\end{array}\right\}=\left\{\begin{array}{c}\boldsymbol{e}_{\boldsymbol{\Gamma}_{u}} \\ -\boldsymbol{Q}_{\Omega} \\ -\boldsymbol{Q}_{\boldsymbol{\Gamma}_{t}}\end{array}\right\}$

onde

$F=\int_{\Omega} S_{\Omega}^{\mathrm{T}} f S_{\Omega} d \Omega$

$A_{\Omega}=\int_{\Omega}\left(L S_{\Omega}\right)^{\mathrm{T}} U_{\Omega} d \Omega$

$A_{\Gamma_{t}}=\int_{\Gamma_{\mathrm{t}}}\left(\mathrm{N} S_{\Omega}\right)^{\mathrm{T}} U_{\Gamma_{t}} d \Gamma$

$e_{\Gamma_{u}}=\int_{\Gamma_{\mathbf{u}}}\left(\mathbf{N} S_{\Omega}\right)^{\mathrm{T}} \bar{u} d \Gamma$

$Q_{\Omega}=\int_{\Omega} U_{\Omega}^{\mathrm{T}} \bar{b} d \Omega$

$Q_{\Gamma_{t}}=\int_{\Gamma_{t}} U_{\Gamma_{t}}^{\mathrm{T}} \bar{t} d \Gamma$

Ao se introduzir a discretização do volume $\boldsymbol{\Omega}$ em elementos, para cada um definem-se aproximações aos campos independentes de tensão e deslocamento. Assim, dentro do domínio do problema aparece uma nova parte do contorno, chamada de contorno interno ou entre elementos $\left(\boldsymbol{\Gamma}_{\boldsymbol{i}}\right)$. Neste, os campos de deslocamentos e forças precisam ser compatibilizados com os campos internos dos elementos adjacentes. Assim sendo, individualizam-se novas incógnitas que podem ser aproximadas como campos independentes em $\Gamma_{i}$.

Em geral, as variáveis discretizáveis para o elemento de volume $\boldsymbol{\Omega}$ e contornos $\boldsymbol{\Gamma}_{\boldsymbol{t}}, \boldsymbol{\Gamma}_{\boldsymbol{u}} \mathrm{e}$ $\Gamma_{i}$ (Figura 3.1), são: 


$$
\begin{array}{ll}
\boldsymbol{\sigma}=\boldsymbol{S}_{\boldsymbol{\Omega}} \boldsymbol{s}_{\boldsymbol{\Omega}} & , \mathrm{em} \boldsymbol{\Omega} \\
\boldsymbol{u}=\boldsymbol{U}_{\boldsymbol{\Omega}} \boldsymbol{q}_{\boldsymbol{\Omega}} & , \mathrm{em} \boldsymbol{\Omega} \\
\boldsymbol{u}_{\boldsymbol{\Gamma}}=\boldsymbol{U}_{\boldsymbol{\Gamma}_{\boldsymbol{t}}} \boldsymbol{q}_{\boldsymbol{\Gamma}_{\boldsymbol{t}}}, \mathrm{em} \boldsymbol{\Gamma}_{\boldsymbol{t}} \\
\boldsymbol{t}_{\boldsymbol{\Gamma}_{\boldsymbol{u}}}=\boldsymbol{W}_{\boldsymbol{\Gamma}_{\boldsymbol{u}}} \boldsymbol{p}_{\boldsymbol{\Gamma}_{\boldsymbol{u}}} \quad, \mathrm{em} \boldsymbol{\Gamma}_{\boldsymbol{u}} \\
\boldsymbol{u}_{\boldsymbol{\Gamma}_{\boldsymbol{i}}}=\boldsymbol{U}_{\boldsymbol{\Gamma}_{\boldsymbol{i}}} \boldsymbol{q}_{\boldsymbol{\Gamma}_{\boldsymbol{i}}} \quad, \mathrm{em} \boldsymbol{\Gamma}_{\boldsymbol{i}} \\
\boldsymbol{t}_{\boldsymbol{\Gamma}_{\boldsymbol{i}}}=\boldsymbol{W}_{\boldsymbol{\Gamma}_{\boldsymbol{i}}} \boldsymbol{p}_{\boldsymbol{\Gamma}_{\boldsymbol{i}}} \quad, \mathrm{em} \boldsymbol{\Gamma}_{\boldsymbol{i}}
\end{array}
$$

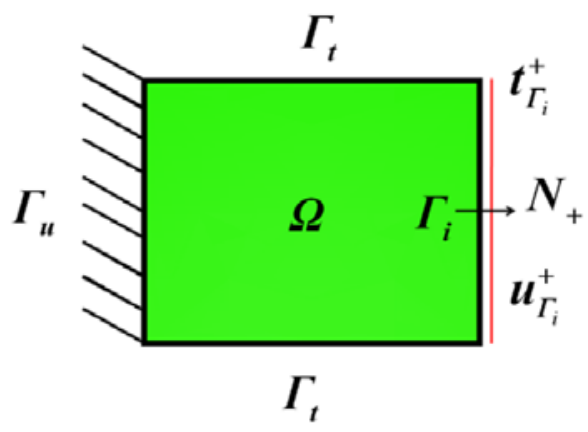

Figura 3.1 - Elemento de volume $\boldsymbol{\Omega}$ e contornos $\boldsymbol{\Gamma}_{\boldsymbol{t}}, \boldsymbol{\Gamma}_{\boldsymbol{u}}$ e $\boldsymbol{\Gamma}_{\boldsymbol{i}}$. Fonte: Góis (2009).

Em razão da existência de $\boldsymbol{\Gamma}_{\boldsymbol{i}}$ no elemento, as condições de compatibilidade e equilíbrio são então estendidas de modo a incluir os novos campos e condições sobre esse contorno. Assim, o sistema resolvente fica dado por:

$$
\left[\begin{array}{cccc}
\boldsymbol{F} & \boldsymbol{A}_{\Omega} & -\boldsymbol{A}_{\boldsymbol{\Gamma}_{t}} & -\boldsymbol{A}_{\boldsymbol{\Gamma}_{i}} \\
\boldsymbol{A}_{\Omega}^{\mathrm{T}} & \mathbf{0} & \mathbf{0} & \mathbf{0} \\
-\boldsymbol{A}_{\boldsymbol{\Gamma}_{t}}^{\mathrm{T}} & \mathbf{0} & \mathbf{0} & \mathbf{0} \\
-\boldsymbol{A}_{\boldsymbol{\Gamma}_{i}}^{\mathrm{T}} & \mathbf{0} & \mathbf{0} & \mathbf{0}
\end{array}\right]\left\{\begin{array}{c}
\boldsymbol{s}_{\Omega} \\
\boldsymbol{q}_{\Omega} \\
\boldsymbol{q}_{\Gamma_{t}} \\
\boldsymbol{q}_{\Gamma_{i}}^{+}
\end{array}\right\}=\left\{\begin{array}{c}
\boldsymbol{e}_{\Gamma_{u}} \\
-\boldsymbol{Q}_{\Omega} \\
-\boldsymbol{Q}_{\Gamma_{t}} \\
-\boldsymbol{Q}_{\Gamma_{i}}^{+}
\end{array}\right\}
$$

onde 


$$
\begin{aligned}
& A_{\Gamma_{i}}=\int_{\Gamma_{\mathrm{i}}}\left(\mathrm{N} S_{\Omega}\right)^{\mathrm{T}} U_{\Gamma_{i}} d \Gamma \\
& Q_{\Gamma_{i}}^{+}=\int_{\Gamma_{t}} U_{\Gamma_{i}}^{\mathrm{T}} t_{\Gamma_{i}}^{+} d \Gamma
\end{aligned}
$$

Nota-se, na equação de equilíbrio em $\boldsymbol{\Gamma}_{\boldsymbol{i}}$, que $\boldsymbol{t}_{\boldsymbol{\Gamma}_{\boldsymbol{i}}}^{+}$também é desconhecida. Naturalmente o sistema descrito pode se apresentar com características particulares, por exemplo, a parte de contorno $\boldsymbol{\Gamma}_{\boldsymbol{i}}$ simplesmente não existe numa discretização com um único elemento. Por outro lado, em se tratando de elemento interior, as partes $\boldsymbol{\Gamma}_{\boldsymbol{t}}$ e $\boldsymbol{\Gamma}_{\boldsymbol{u}}$ não existem. Nesses casos, os sistemas correspondentes apresentam matrizes com diferentes ordens e diferente número de variáveis.

De acordo com Proença (2007), Ao considerar um mosaico composto de elementos, sua união exige a imposição de condições de continuidade de deslocamentos e de equilíbrio de forças nos contornos internos. Entretanto, com o objetivo de reduzir o número de incógnitas quando da união do mosaico de elementos, é usual a imposição em forma forte de uma dessas condições, impondo-se a outra em forma fraca.

A condição sobre os deslocamentos é dita de continuidade, sendo expressa em forma forte em $\boldsymbol{\Gamma}_{\boldsymbol{i}}$ comum por: $\boldsymbol{u}_{\boldsymbol{\Gamma}_{\boldsymbol{i}}}^{+}=\boldsymbol{u}_{\boldsymbol{\Gamma}_{\boldsymbol{i}}}^{-}$. A condição sobre as forças é dita de reciprocidade, sendo expressa no contorno comum por: $\boldsymbol{t}_{\boldsymbol{\Gamma}_{i}}^{+}=\boldsymbol{t}_{\boldsymbol{\Gamma}_{i}}^{-}$. De acordo com a hipótese que venha a ser admitida em $\boldsymbol{\Gamma}_{\boldsymbol{i}}$, a outra deverá ser imposta em forma fraca, passando esta fronteira a ser entendida como um contorno de Neumann (estático) ou de Dirichlet (cinemático).

Para entender melhor as hipóteses de compatibilização a serem adotadas entre elementos, é importante analisar, inicialmente, a composição e o significado de cada uma das equações do sistema apresentado para um único elemento.

Em primeiro lugar, vale salientar que as relações indicadas decorrem de ponderações sobre as equações de compatibilidade e equilíbrio no domínio, e equilíbrio nas partes $\boldsymbol{\Gamma}_{\boldsymbol{t}}$ e $\boldsymbol{\Gamma}_{\boldsymbol{i}}$ do contorno. Excetuando-se a condição de contorno dos pontos pertencentes à $\boldsymbol{\Gamma}_{\boldsymbol{u}}$, que é satisfeita em forma forte.

A primeira equação tem origem na integração por partes da condição de compatibilidade entre deslocamentos e deformações do elemento, o que acaba por inserir também a compatibilização com os campos de deslocamentos nas partes $\boldsymbol{\Gamma}_{\boldsymbol{t}}$ e $\boldsymbol{\Gamma}_{\boldsymbol{i}}$ do contorno. 
Formulações não convencionais em elementos finitos

Levando-se em conta, por um lado, que os campos de deslocamentos no domínio e no contorno são representados por aproximações independentes e, por outro, que o contorno se divide em regiões distintas, tal integração fornece:

$$
\begin{aligned}
\int_{\Omega} S_{\Omega}^{\mathrm{T}} f \sigma d \Omega & +\int_{\Omega}\left(\mathbf{L S}_{\Omega}\right)^{\mathrm{T}} u d \Omega-\int_{\Gamma_{t}}\left(\mathrm{NS}_{\Omega}\right)^{\mathrm{T}} u_{\Gamma_{t}} d \Gamma-\int_{\Gamma_{i}}\left(\mathrm{~N}_{+} \mathbf{S}_{\Omega}\right)^{\mathrm{T}} u_{\Gamma_{i}} d \Gamma= \\
& =\int_{\Gamma_{u}}\left(\mathrm{NM}_{\Omega}\right)^{\mathrm{T}} \bar{u} d \Gamma
\end{aligned}
$$

Dessa condição, introduzindo-se as aproximações para as tensões e deslocamentos no domínio e deslocamentos nos contornos $\boldsymbol{\Gamma}_{\boldsymbol{t}}$ e $\boldsymbol{\Gamma}_{\boldsymbol{i}}$, obtém-se a primeira equação do sistema:

$$
F s_{\Omega}+A_{\Omega} q_{\Omega}-A_{\Gamma_{t}} q_{\Gamma_{t}}-A_{\Gamma_{i}} q_{\Gamma_{i}}^{+}=e_{\Gamma_{u}}
$$

Outra relação que aparece no exemplo considerado é a que exprime o equilíbrio na fronteira $\boldsymbol{\Gamma}_{\boldsymbol{i}}$.

$$
\int_{\Gamma} U_{\Gamma_{i}}^{T}\left(t_{\Gamma_{i}}^{+}-\mathbf{N}_{+} \sigma\right) d \Gamma=0
$$

Substituindo-se nessa condição a aproximação para o campo de tensões no domínio, resulta a quarta equação do sistema do elemento:

$$
A_{\Gamma_{i}}^{\mathrm{T}} s_{\Omega}-Q_{\Gamma_{i}}^{+}=\mathbf{0}
$$

As outras equações (segunda e terceira) referem-se ao equilíbrio no volume e à condição de Neumann no contorno $\boldsymbol{\Gamma}_{\boldsymbol{t}}$, sendo de interpretação mais simples. É importante observar, neste ponto, que o sistema indicado para um elemento apresenta quatro equações e cinco incógnitas.

Considere-se, agora, o exemplo de dois elementos, indicado na Figura 3.2. Se nenhuma hipótese de equilíbrio (reciprocidade) ou de continuidade na fronteira comum é adotada a priori, as seguintes aproximações são válidas no contorno $\boldsymbol{\Gamma}_{\boldsymbol{i}}$ de cada elemento: 
$\boldsymbol{u}_{\Gamma_{i}}^{+}=U_{\Gamma_{i}}^{+} \boldsymbol{q}_{\Gamma_{i}}^{+}, \boldsymbol{t}_{\Gamma_{i}}^{+}=\boldsymbol{W}_{\Gamma_{i}}^{+} \boldsymbol{p}_{\boldsymbol{\Gamma}_{i}}^{+}, \boldsymbol{u}_{\boldsymbol{\Gamma}_{i}}^{-}=\boldsymbol{U}_{\boldsymbol{\Gamma}_{i}}^{-} \boldsymbol{q}_{\boldsymbol{\Gamma}_{i}}^{-}$e $\boldsymbol{t}_{\boldsymbol{\Gamma}_{i}}^{-}=\boldsymbol{W}_{\boldsymbol{\Gamma}_{i}}^{-} \boldsymbol{p}_{\boldsymbol{\Gamma}_{\boldsymbol{i}}}^{-}$. Assim sendo, nesta situação geral, $\boldsymbol{q}_{\Gamma_{i}}^{+}, \boldsymbol{p}_{\Gamma_{i}}^{+}, \boldsymbol{q}_{\boldsymbol{\Gamma}_{i}}^{-}$e $\boldsymbol{p}_{\boldsymbol{\Gamma}_{i}}^{-}$são incógnitas adicionais a determinar. Dada à independência adotada para os campos envolvidos resulta um total de oito equações e dez incógnitas envolvidas, ao considerar o sistema de equações dos dois elementos (Figura 3.2). As duas equações adicionais para tornar o sistema determinado são, justamente, de continuidade e reciprocidade na fronteira comum.

A imposição em forma forte de uma das condições, de continuidade ou de equilíbrio, permite substituir na fronteira $\boldsymbol{\Gamma}_{\boldsymbol{i}}$ as duas incógnitas, em deslocamentos ou forças, respectivamente, por uma incógnita comum. Com isso, o sistema resolvente passa a apresentar nove incógnitas e nove equações, sendo montado primeiramente com as condições sobre cada elemento referentes à compatibilidade e equilíbrio no volume e equilíbrio nos contornos $\boldsymbol{\Gamma}_{\boldsymbol{t}}$ e $\boldsymbol{\Gamma}_{\boldsymbol{i}}$, dispostas em sequência, sem sobreposição. Há, ainda, uma equação adicional relativa à forma fraca da outra condição, de reciprocidade ou de continuidade, envolvendo as incógnitas em forças ou deslocamentos, respectivamente, no contorno comum $\boldsymbol{\Gamma}_{\boldsymbol{i}}$

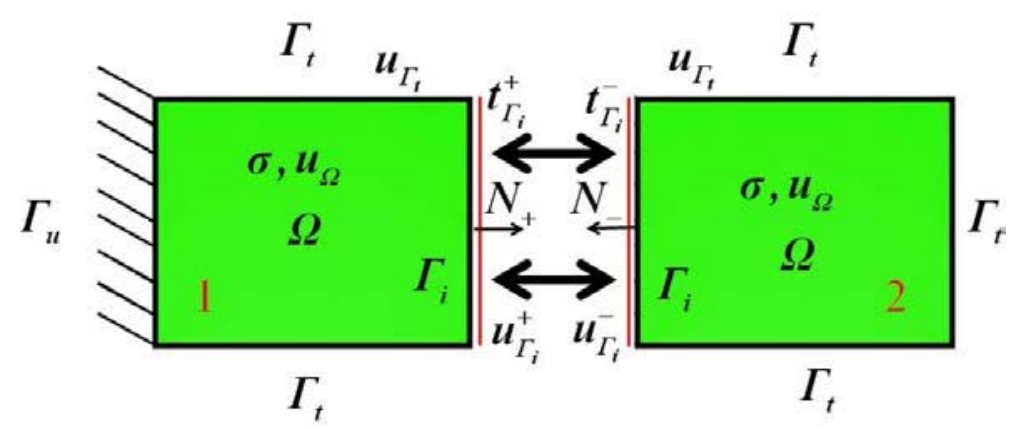

Figura 3.2 - Dois elementos de volume $\boldsymbol{\Omega}$ e contornos $\boldsymbol{\Gamma}_{\boldsymbol{t}}, \boldsymbol{\Gamma}_{\boldsymbol{u}}$ e $\boldsymbol{\Gamma}_{\boldsymbol{i}}$. Fonte: Góis (2009).

Seja uma primeira hipótese que na fronteira comum $\boldsymbol{\Gamma}_{\boldsymbol{i}}$ haja reciprocidade de forças incógnitas entre elementos, isto é: $\boldsymbol{t}_{\boldsymbol{\Gamma}_{i}}^{+}=-\boldsymbol{t}_{\boldsymbol{\Gamma}_{\boldsymbol{i}}}^{-}=\boldsymbol{t}=\boldsymbol{W}_{\boldsymbol{\Gamma}_{i}} \boldsymbol{p}_{\boldsymbol{\Gamma}_{\boldsymbol{i}}}$. Segue que a condição de continuidade passa a ser imposta em forma ponderada. Nesta situação $\boldsymbol{\Gamma}_{\boldsymbol{i}}$ pode ser interpretado como um contorno cinemático ou de Dirichlet. Portanto, o número de incógnitas em $\boldsymbol{\Gamma}_{\boldsymbol{i}}$ se reduz a: $\boldsymbol{q}_{\Gamma_{i}}^{+}, \boldsymbol{q}_{\boldsymbol{\Gamma}_{\boldsymbol{i}}}^{-}$e $\boldsymbol{p}_{\boldsymbol{\Gamma}_{\boldsymbol{i}}}$.

Inicialmente, a condição de reciprocidade das forças na fronteira $\boldsymbol{\Gamma}_{\boldsymbol{i}}$ introduz alguma modificação nas relações referentes ao equilíbrio na fronteira comum, abaixo reproduzidas: 


$$
\begin{aligned}
& \left(A_{\Gamma_{i}}^{+}\right)^{\mathrm{T}}\left(s_{\Omega}\right)^{1}=Q_{\Gamma_{i}}^{+} \\
& \left(A_{\Gamma_{i}}^{-}\right)^{\mathrm{T}}\left(s_{\Omega}\right)^{2}=Q_{\Gamma_{i}}^{-}
\end{aligned}
$$

Pela reciprocidade admitida, cada termo correspondente à forma linear, isto é: $\boldsymbol{Q}_{\mathbf{\Gamma}_{i}}^{+}$e $\boldsymbol{Q}_{\Gamma_{i}}^{-}$, passam a ser escritos como:

$$
\begin{aligned}
& Q_{\Gamma_{i}}^{+}=\int_{\Gamma_{i}^{+}}\left(U_{\Gamma_{i}}^{+}\right)^{\mathrm{T}} t d \Gamma \\
& Q_{\Gamma_{i}}^{-}=-\int_{\Gamma_{i}^{-}}\left(U_{\Gamma_{i}}^{-}\right)^{\mathrm{T}} t d \Gamma
\end{aligned}
$$

Considerando-se a aproximação adotada para a força $\boldsymbol{t}$, essas formas lineares passam a ter novas redações. Assim, para o elemento '1' (Figura 3.2), tem-se:

$$
\begin{gathered}
\boldsymbol{Q}_{\Gamma_{i}}^{+}=\int_{\Gamma_{i}^{+}}\left(U_{\Gamma_{i}}^{+}\right)^{\mathrm{T}} t d \Gamma=\int_{\Gamma_{i}^{+}}\left(U_{\Gamma_{i}}^{+}\right)^{\mathrm{T}} W_{\Gamma_{i}} p_{\Gamma_{i}} d \Gamma=\left[\int_{\Gamma_{i}^{+}}\left(U_{\Gamma_{i}}^{+}\right)^{\mathrm{T}} \boldsymbol{W}_{\Gamma_{i}} d \Gamma\right] p_{\Gamma_{i}}= \\
=\left(C_{\Gamma_{i}}^{+}\right)^{\mathrm{T}} \boldsymbol{p}_{\Gamma_{i}}
\end{gathered}
$$

Analogamente, para o elemento '2' (Figura 3.2) tem-se:

$$
\begin{gathered}
\boldsymbol{Q}_{\Gamma_{i}}^{-}=\int_{\Gamma_{i}^{-}}\left(U_{\Gamma_{i}}^{-}\right)^{\mathrm{T}} t d \Gamma=\int_{\Gamma_{i}^{-}}\left(U_{\Gamma_{i}}^{-}\right)^{\mathrm{T}} W_{\Gamma_{i}} p_{\Gamma_{i}} d \Gamma=\left[\int_{\Gamma_{i}^{-}}\left(U_{\Gamma_{i}}^{-}\right)^{\mathrm{T}} W_{\Gamma_{i}} d \Gamma\right] p_{\Gamma_{i}}= \\
=\left(C_{\Gamma_{i}}^{-}\right)^{\mathrm{T}} p_{\Gamma_{i}}
\end{gathered}
$$

Com isso, as relações de equilíbrio na fronteira comum passam a ser escritas como:

$$
\left(A_{\Gamma_{i}}^{+}\right)^{\mathrm{T}}\left(s_{\Omega}\right)^{1}-\left(C_{\Gamma_{i}}^{+}\right)^{\mathrm{T}} \boldsymbol{p}_{\Gamma_{i}}=\mathbf{0} \quad \text { [elemento '1'] }
$$




$$
\left(\boldsymbol{A}_{\boldsymbol{\Gamma}_{i}}^{-}\right)^{\mathrm{T}}\left(\boldsymbol{s}_{\Omega}\right)^{2}+\left(\boldsymbol{C}_{\boldsymbol{\Gamma}_{i}}^{-}\right)^{\mathrm{T}} \boldsymbol{p}_{\boldsymbol{\Gamma}_{i}}=\mathbf{0} \quad \text { [elemento '2'] }
$$

Em relação à representação da continuidade dos deslocamentos em $\boldsymbol{\Gamma}_{\boldsymbol{i}}$, em forma fraca, tal condição pode ser imposta tomando-se forças virtuais como ponderadoras, sendo, então, expressa como:

$$
\int_{\Gamma_{i}} \delta t^{\mathrm{T}}\left(u_{\Gamma_{i}}^{+}-u_{\Gamma_{i}}^{-}\right) d \Gamma=0
$$

Considerando-se que as forças virtuais possam ser escritas como: $\delta \boldsymbol{t}=\boldsymbol{W}_{\boldsymbol{\Gamma}_{\boldsymbol{i}}} \delta \boldsymbol{p}_{\boldsymbol{\Gamma}_{\boldsymbol{i}}}$, a condição anterior passa a:

$$
\begin{aligned}
& \delta p_{\Gamma_{i}} \int_{\Gamma_{i}} W_{\Gamma_{i}}\left(u_{\Gamma_{i}}^{+}-u_{\Gamma_{i}}^{-}\right) d \Gamma=0 \\
& \int_{\Gamma_{i}} W_{\Gamma_{i}} u_{\Gamma_{i}}^{+} d \Gamma-\int_{\Gamma_{i}} W_{\Gamma_{i}} u_{\Gamma_{i}}^{-} d \Gamma=0 \\
& {\left[\int_{\Gamma_{i}} W_{\Gamma_{i}} U_{\Gamma_{i}}^{+} d \Gamma\right] q_{\Gamma_{i}}^{+}-\left[\int_{\Gamma_{i}} W_{\Gamma_{i}} U_{\Gamma_{i}}^{-} d \Gamma\right] q_{\Gamma_{i}}^{-}=0} \\
& C_{\Gamma_{i}}^{+} q_{\Gamma_{i}}^{+}-C_{\Gamma_{i}}^{-} q_{\Gamma_{i}}^{-}=0
\end{aligned}
$$

Portanto, de acordo com essa hipótese de compatibilização (em deslocamentos), o sistema resolvente para dois elementos fica composto pelas contribuições das relações de compatibilidade e equilíbrio no volume e equilíbrios nos contornos $\boldsymbol{\Gamma}_{\boldsymbol{t}}$ e $\boldsymbol{\Gamma}_{\boldsymbol{i}}$ para cada elemento, mais a relação de continuidade dos deslocamentos na fronteira comum. Tal sistema apresentase na eq.(3.51), onde $\boldsymbol{e}_{\Gamma_{u}}^{\mathbf{1}}$ e $\boldsymbol{e}_{\boldsymbol{\Gamma}_{\boldsymbol{u}}}^{\mathbf{2}}$ existem se, e somente se, os respectivos elementos possuírem partes de seus contornos contidas no contorno global de Dirichlet e com restrições não nulas nos deslocamentos. 


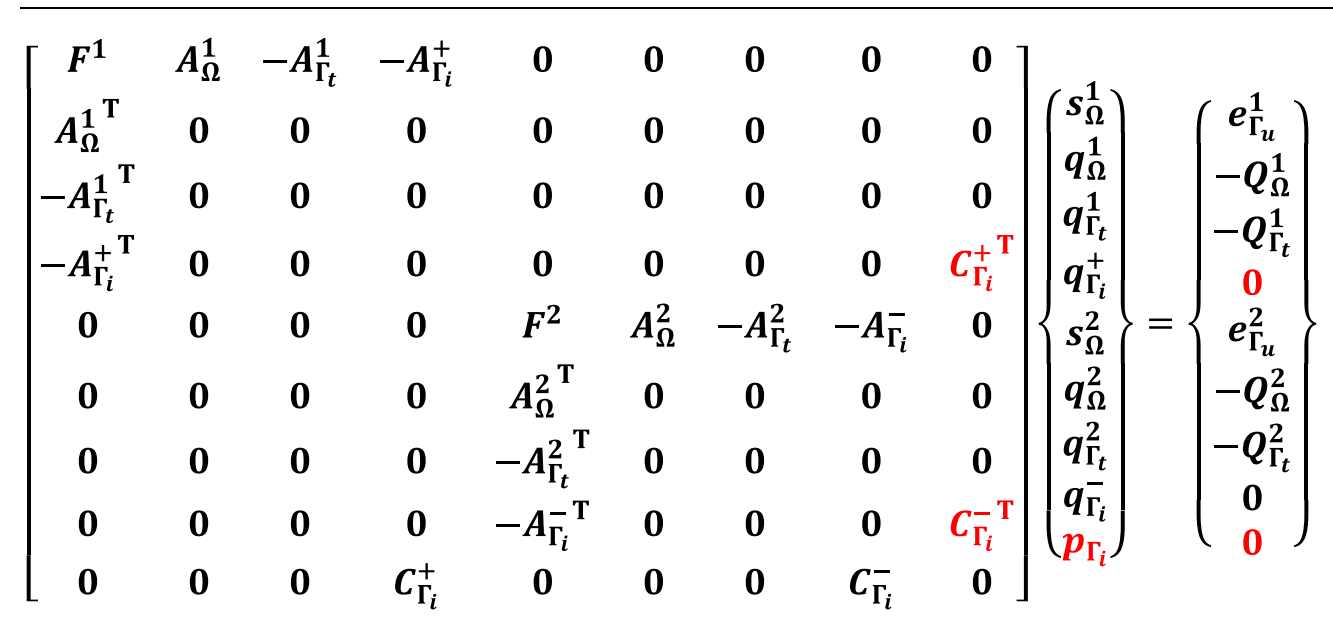

A segunda hipótese consiste em impor a priori a continuidade de deslocamentos na fronteira comum, valendo: $\boldsymbol{u}_{\Gamma_{i}}^{+}=\boldsymbol{u}_{\Gamma_{i}}^{-}=\boldsymbol{u}_{\Gamma_{i}}=\boldsymbol{U}_{\Gamma_{i}} \boldsymbol{q}_{\Gamma_{i}}$. A reciprocidade de forças passa a ser imposta em forma ponderada. Portanto, nessa hipótese $\boldsymbol{\Gamma}_{\boldsymbol{i}}$ pode ser entendida como uma fronteira de Neumann.

Em razão da continuidade de deslocamentos, a primeira equação de cada elemento (Figura 3.2) tem seu termo associado à fronteira $\boldsymbol{\Gamma}_{\boldsymbol{i}}$ sofrendo uma pequena modificação, uma vez que as variáveis da aproximação para o campo de deslocamentos na fronteira comum entre elementos não mais se distinguem. Assim sendo, resulta:

$$
\begin{gathered}
\int_{\Gamma_{i}^{+}}\left(\mathrm{N}_{+} S_{\Omega}^{1}\right)^{\mathrm{T}} u_{\Gamma_{i}}^{+} d \Gamma=\left[\int_{\Gamma_{i}^{+}}\left(\mathrm{N}_{+} S_{\Omega}^{1}\right)^{\mathrm{T}} U_{\Gamma_{i}} d \Gamma\right] q_{\Gamma_{i}}=A_{\Gamma_{i}}^{+} q_{\Gamma_{i}} \\
\int_{\Gamma_{i}^{-}}\left(\mathrm{N}_{-} S_{\Omega}^{2}\right)^{\mathrm{T}} u_{\Gamma_{i}}^{-} d \Gamma=-\left[\int_{\Gamma_{i}^{-}}\left(\mathrm{N}_{-} S_{\Omega}^{2}\right)^{\mathrm{T}} U_{\Gamma_{i}} d \Gamma\right] q_{\Gamma_{i}}=-A_{\Gamma_{i}}^{-} q_{\Gamma_{i}}
\end{gathered}
$$

A equação adicional, de reciprocidade de forças na fronteira $\boldsymbol{\Gamma}_{\boldsymbol{i}}$, é imposta em forma ponderada. Tal condição escreve-se:

$$
\int_{\Gamma_{i}} \delta u_{\Gamma_{i}}^{\mathrm{T}}\left(t_{\Gamma_{i}}^{+}+t_{\Gamma_{i}}^{-}\right) d \Gamma=0
$$

Substituindo-se $\delta \boldsymbol{u}_{\boldsymbol{\Gamma}_{i}}^{\mathrm{T}}=\delta \boldsymbol{q}_{\boldsymbol{\Gamma}_{i}}^{\mathrm{T}} \boldsymbol{U}_{\boldsymbol{\Gamma}_{i}}^{\mathrm{T}}$ resultam, sucessivamente: 
$\delta q_{\Gamma_{i}}^{\mathrm{T}} \int_{\Gamma_{i}} U_{\Gamma_{i}}^{\mathrm{T}}\left(t_{\Gamma_{i}}^{+}+t_{\Gamma_{i}}^{-}\right) d \Gamma=0$

$\int_{\Gamma_{i}} U_{\Gamma_{i}}^{\mathrm{T}} t_{\Gamma_{i}}^{+} d \Gamma+\int_{\Gamma_{i}} U_{\Gamma_{i}}^{\mathrm{T}} t_{\Gamma_{i}}^{-} d \Gamma=0$

Uma vantagem desta alternativa é a utilização da condição de equilíbrio no contorno, considerando-se o regime de tensões no interior de cada elemento, como forma de redução do número de incógnitas. Assim, introduzindo-se a condição de equilíbrio no contorno $\boldsymbol{\Gamma}_{\boldsymbol{i}}$ comum a cada um dos elementos, segue que:

$$
\begin{aligned}
& \int_{\Gamma_{i}} U_{\Gamma_{i}}^{\mathrm{T}} \mathrm{N}_{+} \sigma_{\Omega}^{1} d \Gamma+\int_{\Gamma_{i}} U_{\Gamma_{i}}^{\mathrm{T}} \mathrm{N}_{-} \sigma_{\Omega}^{2} d \Gamma=0 \\
& {\left[\int_{\Gamma_{i}} U_{\Gamma_{i}}^{\mathrm{T}} \mathrm{N}_{+} S_{\Omega}^{1} d \Gamma\right] s_{\Omega}^{1}+\left[\int_{\Gamma_{i}} U_{\Gamma_{i}}^{\mathrm{T}} \mathrm{N}_{-} S_{\Omega}^{2} d \Gamma\right] s_{\Omega}^{2}=0} \\
& \left(A_{\Gamma_{i}}^{+}\right)^{\mathrm{T}} s_{\Omega}^{1}+\left(A_{\Gamma_{i}}^{-}\right)^{\mathrm{T}} s_{\Omega}^{2}=0
\end{aligned}
$$

Portanto, de acordo com essa hipótese, o sistema resolvente fica composto pelas contribuições das relações de compatibilidade e equilíbrio no volume e equilíbrio no contorno $\boldsymbol{\Gamma}_{\boldsymbol{t}}$, de cada elemento, mais as relações de reciprocidade de forças na fronteira comum $\boldsymbol{\Gamma}_{\boldsymbol{i}}$. Tal sistema apresenta-se na forma:

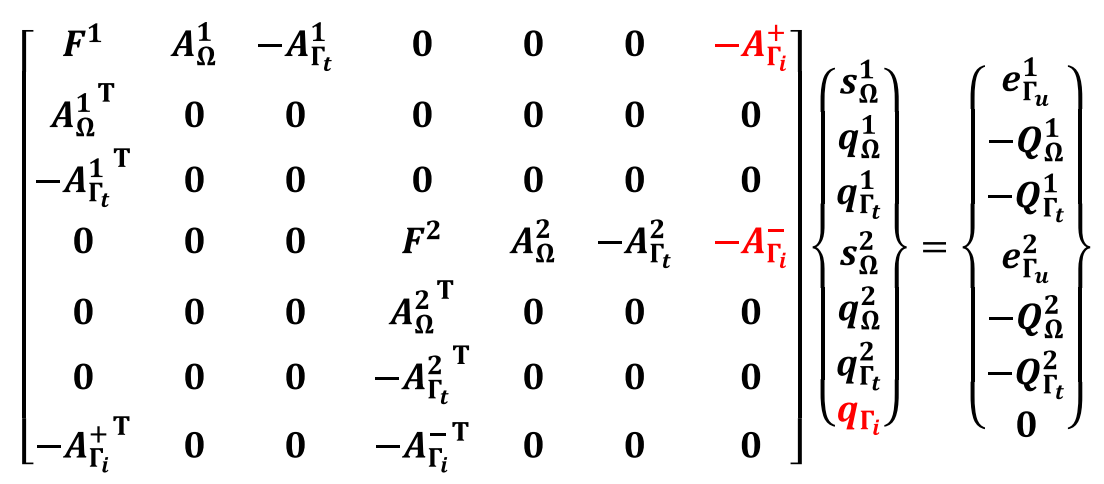


Formulações não convencionais em elementos finitos

Nota-se que de acordo com a hipótese adotada a fronteira $\boldsymbol{\Gamma}_{\boldsymbol{i}}$ em cada elemento passa a ser entendida como um contorno de Dirichlet (cinemático) ou de Neumann (estático). Na primeira hipótese considerada, ao se admitir a priori a reciprocidade de forças entre elementos, $\boldsymbol{\Gamma}_{\boldsymbol{i}}$ passou a ser interpretado como um contorno cinemático. Porque a equação adicional foi de continuidade em deslocamento, a formulação resultante é dita Híbrido-Mista de Deslocamento (FHMD).

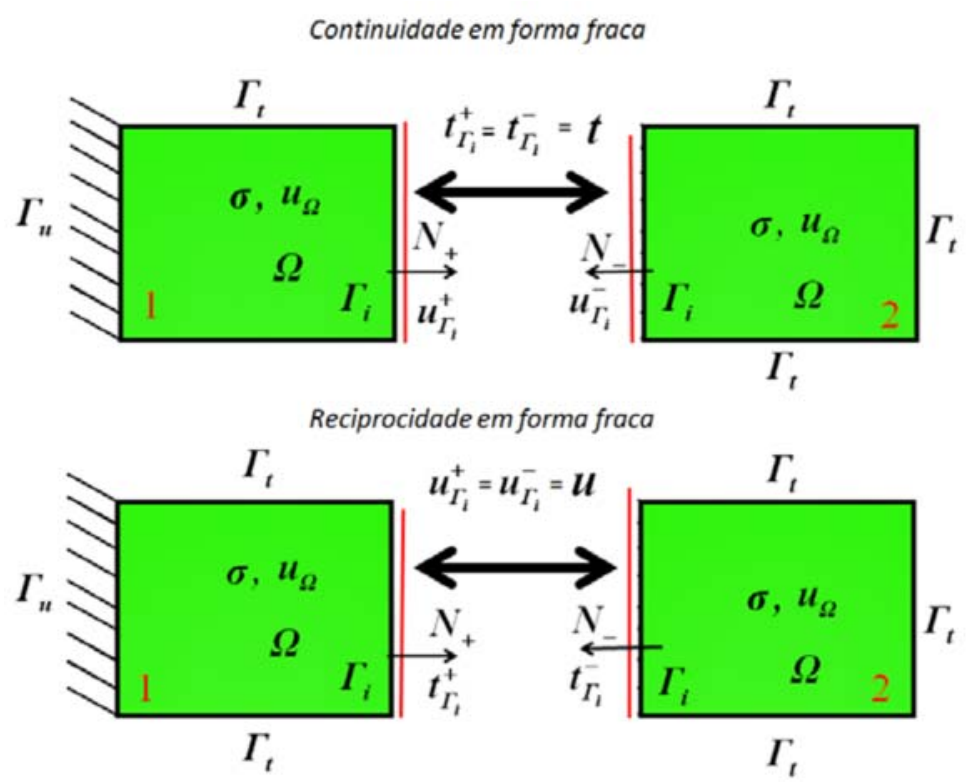

Figura 3.3 - Dois elementos de volume $\boldsymbol{\Omega}$ e contornos $\boldsymbol{\Gamma}_{\boldsymbol{t}}, \boldsymbol{\Gamma}_{\boldsymbol{u}}$ e $\boldsymbol{\Gamma}_{\boldsymbol{i}}$ - continuidade e reciprocidade em forma fraca. Fonte: Góis (2009).

$\mathrm{Na}$ segunda hipótese, onde se admitiu a continuidade de deslocamentos entre elementos, a interpretação para $\boldsymbol{\Gamma}_{\boldsymbol{i}}$ passou a ser de um contorno estático. Porque a equação adicional foi de reciprocidade de forças, a formulação resultante é dita Híbrido-Mista de Tensão (FHMT).

\subsection{Forma Mista u- $\sigma$ para elasticidade plana}

Neste item apresenta-se uma formulação puramente mista para Mecânica dos Sólidos e das Estruturas. Esta forma mista é apresentada por Zienkiewicz e Taylor (2000) considerando que deslocamentos prescritos e forças de volume são nulos. Com isso, este trabalho faz uma abordagem mais geral, para que seja possível a consideração destas grandezas.

Assim, neste desenvolvimento também se admite que as condições no contorno de Dirichlet (em deslocamentos) sejam atendidas em forma forte e todas as outras relações são 
impostas em forma fraca, mediante ponderações. Na primeira delas (eq.(3.61)) estão reunidas as condições de compatibilidade e constitutiva, na segunda (eq.(3.62)) a condição de equilíbrio no domínio e as condições de contorno de Neumann (em tensões).

$$
\begin{aligned}
& \int_{\Omega} \delta \mathbf{m}^{\mathrm{T}}\left(\mathbf{L}^{\mathrm{T}} u-f \sigma\right) d \Omega=0 \\
& \int_{\Omega} \delta \mathbf{p}^{\mathrm{T}}(\mathbf{L} \sigma+\mathbf{b}) d \Omega+\int_{\Gamma_{t}} \delta \mathbf{p}^{\mathrm{T}}(\bar{t}-\mathbf{N} \sigma) d \Gamma=0
\end{aligned}
$$

Aplicando-se o Teorema de Green na primeira integral da eq.(3.61), obtém-se:

$$
\int_{\Omega}(\mathbf{L} \delta \mathbf{m})^{\mathrm{T}} u d \Omega+\int_{\Omega} \delta \mathbf{m}^{\mathrm{T}} f \sigma d \Omega-\int_{\Gamma_{t}}(\mathbf{N} \delta \mathbf{m})^{\mathrm{T}} u d \Gamma-\int_{\Gamma_{u}}(\mathbf{N} \delta \mathbf{m})^{\mathrm{T}} \bar{u} d \Gamma=0
$$

$\mathrm{Na}$ relação anterior estão destacados os termos independentes que caracterizam a forma mista: tensões e deslocamentos. Admite-se que os campos de tensão e deslocamento são incompatíveis e, diferentemente da formulação híbrido-mista, não há diferença entre as aproximações no domínio e no contorno do problema, o que caracteriza esta formulação como puramente mista.

As aproximações para os campos incógnitos são dadas a seguir, assim como às ponderações adotadas.

$\sigma=S_{\Omega} s_{\Omega}$

$\boldsymbol{u}=\boldsymbol{U}_{\Omega} \boldsymbol{q}_{\Omega}$

$\delta \mathbf{m}=\mathbf{M}_{\Omega} \delta \mathbf{m}_{\Omega}$

$\delta \mathbf{p}=\mathbf{P}_{\Omega} \delta \mathbf{p}_{\boldsymbol{\Omega}}$ 
Formulações não convencionais em elementos finitos

Nas relações anteriores, o vetor $\boldsymbol{s}_{\boldsymbol{\Omega}}$ pode ser interpretado como o vetor dos parâmetros de tensão associados às funções de aproximação dos campos de tensão em $\boldsymbol{S}_{\boldsymbol{\Omega}}$. Já $\boldsymbol{U}_{\boldsymbol{\Omega}}$ é a matriz que armazena as funções de aproximação do campo de deslocamento e $\boldsymbol{q}_{\boldsymbol{\Omega}}$ é o vetor que agrupa os graus de liberdade generalizados associados.

Substituindo-se essas aproximações no conjunto de relações ponderadas e admitindose que os campos virtuais $\delta \mathbf{m}_{\boldsymbol{\Omega}}$ e $\delta \mathbf{p}_{\boldsymbol{\Omega}}$ são compatíveis e homogêneos nas condições de contorno essenciais, obtém-se o seguinte sistema:

$$
\begin{aligned}
& {\left[\begin{array}{cc}
\int_{\Omega} \mathbf{M}_{\Omega}^{\mathrm{T}}\left(f S_{\Omega}\right) d \Omega & \int_{\Omega}\left(\mathbf{L M}_{\Omega}\right)^{\mathrm{T}} U_{\Omega} d \Omega-\int_{\Gamma_{\mathrm{t}}}\left(\mathrm{NM}_{\Omega}\right)^{\mathrm{T}} U_{\Omega} d \Gamma \\
\int_{\Omega} \mathbf{P}_{\Omega}^{\mathrm{T}}\left(\mathbf{L M}_{\Omega}\right) d \Omega-\int_{\Gamma_{\mathrm{t}}} \mathbf{P}_{\Omega}^{\mathrm{T}}\left(\mathrm{NM}_{\Omega}\right) d \Gamma & 0
\end{array}\right]\left\{\begin{array}{l}
s_{\Omega} \\
q_{\Omega}
\end{array}\right\}} \\
& =\left\{\begin{array}{l}
\boldsymbol{e}_{\boldsymbol{\Gamma}_{u}} \\
-\boldsymbol{Q}
\end{array}\right\}
\end{aligned}
$$

onde

$$
\begin{aligned}
& e_{\Gamma_{u}}=\int_{\Gamma_{\mathbf{u}}}\left(\mathrm{NM}_{\Omega}\right)^{\mathrm{T}} \bar{u} d \Gamma \\
& Q=\int_{\Omega} \mathbf{P}_{\Omega}^{\mathrm{T}} \mathbf{b} d \Omega+\int_{\Gamma_{t}} \mathbf{P}_{\Omega}^{\mathrm{T}} \bar{t} d \Gamma
\end{aligned}
$$

Como se observa, o sistema apresentado não é simétrico. Esta condição pode ser alcançada se forem aplicadas restrições tipo Galerkin sobre o conjunto de funções de aproximação:

$$
\mathbf{M}_{\Omega}=S_{\Omega}
$$

$\mathbf{P}_{\mathbf{\Omega}}=\boldsymbol{U}_{\mathbf{\Omega}}$

Assim, o sistema resolvente passa a apresentar formato simétrico: 
$\left[\begin{array}{cc}F & A \\ A^{T} & 0\end{array}\right]\left\{\begin{array}{l}s_{\Omega} \\ q_{\Omega}\end{array}\right\}=\left\{\begin{array}{c}e_{\Gamma_{u}} \\ -Q\end{array}\right\}$

onde

$F=\int_{\Omega} S_{\Omega}^{\mathrm{T}} f S_{\Omega} d \Omega$

$A=\int_{\Omega}\left(\mathrm{L} S_{\Omega}\right)^{\mathrm{T}} U_{\Omega} d \Omega-\int_{\Gamma_{t}}\left(\mathrm{~N} S_{\Omega}\right)^{\mathrm{T}} U_{\Omega} d \Gamma$

$Q=\int_{\Omega} U_{\Omega}^{\mathrm{T}} \mathrm{b} d \Omega+\int_{\Gamma_{t}} U_{\Omega}^{\mathrm{T}} \bar{t} d \Gamma$

$e_{\Gamma_{u}}=\int_{\Gamma_{\mathbf{u}}}\left(\mathbf{N} S_{\Omega}\right)^{\mathrm{T}} \bar{u} d \Gamma$

Ao se introduzir a discretização do domínio em elementos, em cada um definem-se aproximações para os campos independentes. Assim, ao considerar um mosaico de elementos, a sua união exige a imposição de condições de continuidade de deslocamentos e de equilíbrio de forças entre elementos (Figura 3.4). Usualmente, impõe-se uma das condições em forma forte e a outra em forma fraca.

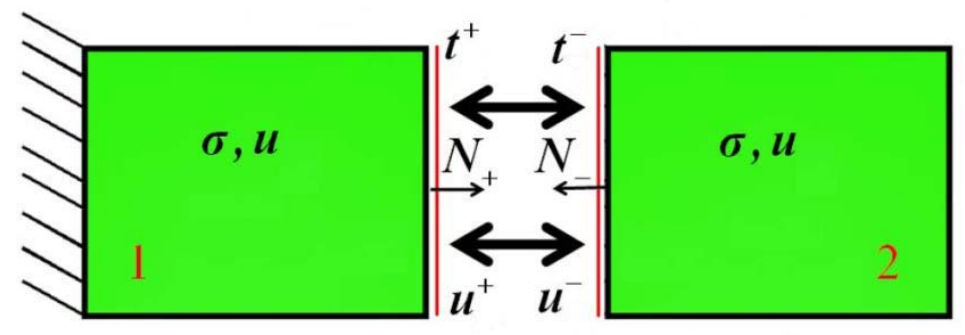

Figura 3.4 - Dois elementos mistos.

Assim, seja uma primeira hipótese que consiste em impor a priori a reciprocidade de forças entre elementos, isto é: $\boldsymbol{t}^{+}=-\boldsymbol{t}^{-}=\boldsymbol{t}=\boldsymbol{W} \boldsymbol{p}$. Com isso, a condição de continuidade nos deslocamentos passa a ser imposta em forma ponderada. 
Inicialmente, a condição de reciprocidade das forças entre elementos introduz relações de equilíbrio na fronteira entre os mesmos:

$\left(A^{+}\right)^{\mathrm{T}} \boldsymbol{s}_{\Omega}^{1}=-Q^{+}$

$\left(A^{-}\right)^{\mathrm{T}} s_{\Omega}^{2}=-Q^{-}$

Pela reciprocidade admitida, cada termo correspondente à forma linear, isto é: $\boldsymbol{Q}^{+}$e $\boldsymbol{Q}^{-}$, passam a ser escritos como:

$Q^{+}=\int_{\Omega}\left(U_{\Omega}^{+}\right)^{\mathrm{T}} \mathbf{b}^{+} d \Omega+\int_{\Gamma_{t}}\left(U_{\Omega}^{+}\right)^{\mathrm{T}} \boldsymbol{t} d \Gamma$

$Q^{-}=\int_{\Omega}\left(U_{\Omega}^{-}\right)^{\mathrm{T}} \mathbf{b}^{-} d \boldsymbol{d}+\int_{\Gamma_{t}}\left(U_{\Omega}^{-}\right)^{\mathrm{T}} \boldsymbol{t} d \Gamma$

Considerando-se a aproximação adotada para a força $\boldsymbol{t}$, essas formas lineares passam a ter novas redações. Assim, para o elemento '1' (Figura 3.4), tem-se:

$$
\begin{aligned}
Q^{+}=\int_{\Omega}\left(U_{\Omega}^{+}\right)^{\mathbf{T}} \mathbf{b}^{+} d \Omega+\int_{\Gamma_{t}}\left(U_{\Omega}^{+}\right)^{\mathrm{T}} W p d \Gamma= \\
=\int_{\Omega}\left(U_{\Omega}^{+}\right)^{\mathrm{T}} \mathbf{b}^{+} d \Omega+\left[\int_{\Gamma_{t}}\left(U_{\Omega}^{+}\right)^{\mathrm{T}} W d \Gamma\right] p=Q_{\Omega}^{+}+\left(C^{+}\right)^{\mathrm{T}} p
\end{aligned}
$$

Analogamente, para o elemento '2' (Figura 3.4) tem-se:

$$
\begin{aligned}
Q^{-}=\int_{\Omega}\left(U_{\Omega}^{-}\right)^{\mathrm{T}} \mathbf{b}^{-} d \Omega+\int_{\Gamma_{t}}\left(U_{\Omega}^{-}\right)^{\mathrm{T}} \boldsymbol{W} p d \Gamma= \\
\quad=\int_{\Omega}\left(U_{\Omega}^{-}\right)^{\mathbf{T}} \mathbf{b}^{-} d \Omega+\left[\int_{\Gamma_{t}}\left(U_{\Omega}^{-}\right)^{\mathbf{T}} W d \Gamma\right] p=Q_{\Omega}^{-}+\left(C^{-}\right)^{\mathbf{T}} p
\end{aligned}
$$

onde $\boldsymbol{Q}_{\boldsymbol{\Omega}}^{+}$e $\boldsymbol{Q}_{\boldsymbol{\Omega}}^{-}$são parcelas de $\boldsymbol{Q}^{+}$e $\boldsymbol{Q}^{-}$, respectivamente, referentes às forças de volume. 
Com isso, as relações de equilíbrio na fronteira comum passam a ser escritas como:

$\left(\boldsymbol{A}^{+}\right)^{\mathrm{T}} \boldsymbol{s}_{\Omega}^{\mathbf{1}}-\left(\boldsymbol{C}^{+}\right)^{\mathrm{T}} \boldsymbol{p}=-\boldsymbol{Q}_{\Omega}^{+} \quad$ (elemento '1')

$\left(\boldsymbol{A}^{-}\right)^{\mathrm{T}} \boldsymbol{s}_{\boldsymbol{\Omega}}^{\mathbf{2}}-\left(\boldsymbol{C}^{-}\right)^{\mathrm{T}} \boldsymbol{p}=-\boldsymbol{Q}_{\mathbf{\Omega}}^{-} \quad$ (elemento '2')

Em relação à representação da continuidade dos deslocamentos em forma fraca, tal condição pode ser imposta tomando-se forças virtuais como ponderadoras, sendo, então, expressa como:

$\int_{\Gamma_{i}} \delta t^{T}\left(u^{+}-u^{-}\right) d \Gamma=0$

considerando-se que as forças virtuais possam ser escritas como: $\delta \boldsymbol{t}=\boldsymbol{W} \delta \boldsymbol{p}$, a condição anterior passa a:

$$
\begin{aligned}
& \delta p^{\mathrm{T}} \int_{\Gamma_{i}} W^{\mathrm{T}}\left(u^{+}-u^{-}\right) d \Gamma=0 \\
& \int_{\Gamma_{i}} W^{\mathrm{T}} u^{+} d \Gamma-\int_{\Gamma_{i}} W^{\mathrm{T}} u^{-} d \Gamma=0 \\
& {\left[\int_{\Gamma_{i}} W^{\mathrm{T}} U_{\Omega}^{+} d \Gamma\right] q_{\Omega}^{+}-\left[\int_{\Gamma_{i}} W^{\mathrm{T}} U_{\Omega}^{-} d \Gamma\right] q_{\Omega}^{-}=0} \\
& C^{+} q_{\Omega}^{+}-C^{-} q_{\Omega}^{-}=0
\end{aligned}
$$

Portanto, de acordo com essa hipótese de compatibilização (em deslocamentos), o sistema resolvente para dois elementos fica composto pelas contribuições das relações de compatibilidade e equilíbrio no volume e equilíbrio na fronteira entre elementos. Tal sistema apresenta-se conforme abaixo, onde $\boldsymbol{e}_{\boldsymbol{\Gamma}_{u}}^{\mathbf{1}}$ e $\boldsymbol{e}_{\boldsymbol{\Gamma}_{\boldsymbol{u}}}^{\mathbf{2}}$ existem se, e somente se, os respectivos 
Formulações não convencionais em elementos finitos

elementos possuírem partes de seus contornos contidas no contorno global de Dirichlet e com restrições não nulas nos deslocamentos.

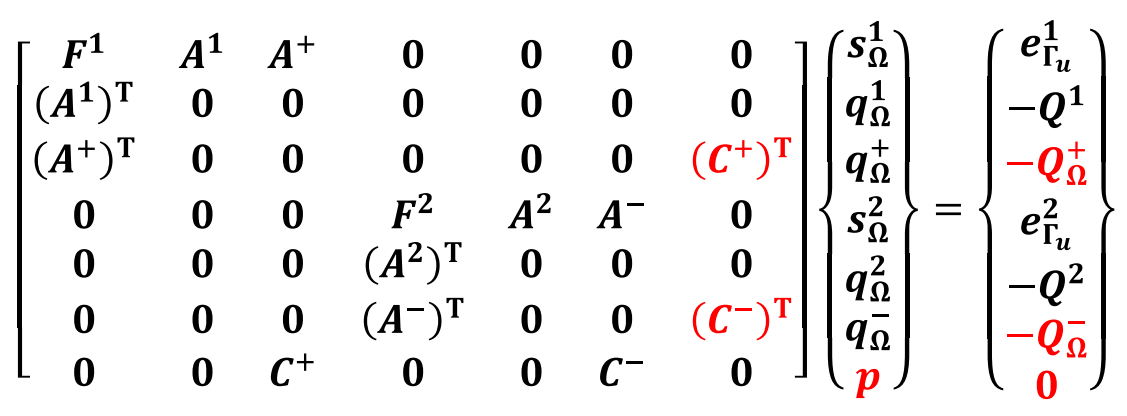

A segunda hipótese consiste em impor a priori a continuidade de deslocamentos entre os elementos, isto é: $\boldsymbol{u}^{+}=\boldsymbol{u}^{-}=\boldsymbol{u}=\boldsymbol{U}_{\Omega} \boldsymbol{q}_{\Omega}$. A reciprocidade de forças passa a ser imposta em forma ponderada.

Em razão da continuidade de deslocamentos, a primeira equação de cada elemento tem seu termo associado ao deslocamento sofrendo uma pequena modificação:

$$
\begin{aligned}
& \int_{\Gamma_{i}^{+}}\left(\mathrm{N}_{+} S_{\Omega}^{1}\right)^{\mathrm{T}} u^{+} d \Gamma=\left[\int_{\Gamma_{i}^{+}}\left(\mathrm{N}_{+} S_{\Omega}^{1}\right)^{\mathrm{T}} U_{\Omega} d \Gamma\right] q_{\Omega}=A_{\Gamma_{i}}^{+} q_{\Omega} \\
& \int_{\Gamma_{i}^{-}}\left(\mathrm{N}_{-} S_{\Omega}^{2}\right)^{\mathrm{T}} u^{-} d \Gamma=-\left[\int_{\Gamma_{i}^{-}}\left(\mathrm{N}_{-} S_{\Omega}^{2}\right)^{\mathrm{T}} U_{\Omega} d \Gamma\right] q_{\Omega}=-A_{\Gamma_{i}}^{-} q_{\Omega}
\end{aligned}
$$

A equação adicional, de reciprocidade de forças, é imposta em forma ponderada:

$$
\int_{\Gamma_{i}} \delta u^{\mathrm{T}}\left(t^{+}+t^{-}\right) d \Gamma=0
$$

Substituindo $\delta \boldsymbol{u}=\boldsymbol{U}_{\boldsymbol{\Omega}} \delta \boldsymbol{q}_{\boldsymbol{\Omega}}$ na, resulta:

$$
\delta \boldsymbol{q}_{\Omega}^{\mathrm{T}} \int_{\Gamma_{i}} U_{\Omega}^{\mathrm{T}}\left(\boldsymbol{t}^{+}+\boldsymbol{t}^{-}\right) d \Gamma=\mathbf{0}
$$


$\int_{\Gamma_{i}} U_{\Omega}^{\mathrm{T}} t^{+} d \Gamma+\int_{\Gamma_{i}} U_{\Omega}^{\mathrm{T}} t^{-} d \Gamma=0$

$\int_{\Gamma_{i}} U_{\Omega}^{\mathrm{T}} \mathrm{N}_{+} \sigma^{1} d \Gamma+\int_{\Gamma_{i}} U_{\Omega}^{\mathrm{T}} \mathbf{N}_{-} \sigma^{2} d \Gamma=0$

$\left[\int_{\Gamma_{i}} U_{\Omega}^{\mathrm{T}} \mathrm{N}_{+} S_{\Omega}^{1} d \Gamma\right] s_{\Omega}^{1}+\left[\int_{\Gamma_{i}} U_{\Omega}^{\mathrm{T}} \mathrm{N}_{-} S_{\Omega}^{2} d \Gamma\right] s_{\Omega}^{2}=0$

$\left(A^{+}\right)^{\mathrm{T}} \boldsymbol{s}_{\Omega}^{1}+\left(A^{-}\right)^{\mathrm{T}} \boldsymbol{s}_{\Omega}^{2}=\mathbf{0}$

Portanto, de acordo com essa hipótese, o sistema resolvente fica conforme a seguir:

$\left[\begin{array}{ccccc}F^{1} & A^{1} & 0 & 0 & A^{+} \\ \left(A^{1}\right)^{T} & 0 & 0 & 0 & 0 \\ 0 & 0 & F^{2} & A^{2} & A^{-} \\ 0 & 0 & \left(A^{2}\right)^{T} & 0 & 0 \\ \left(A^{+}\right)^{T} & 0 & \left(A^{-}\right)^{T} & 0 & 0\end{array}\right]\left\{\begin{array}{c}s_{\Omega}^{1} \\ q_{\Omega}^{1} \\ s_{\Omega}^{2} \\ q_{\Omega}^{2} \\ q_{\Omega}\end{array}\right\}=\left\{\begin{array}{c}e_{\Gamma_{u}}^{1} \\ -Q^{1} \\ e_{\Gamma_{u}}^{2} \\ -Q^{2} \\ 0\end{array}\right\}$

Nota-se que de acordo com a hipótese adotada quanto à continuidade de um dos campos independentes entre elementos ocorrem mudanças no sistema resolvente da forma mista.

$\mathrm{Na}$ primeira hipótese considerada, ao se admitir a priori a reciprocidade de forças entre elementos houve condensação das variáveis de tensão e acréscimo das variáveis de deslocamento. Conforme apresentado por Zienkiewicz e Taylor (2000), o campo de tensões é dito como primário, sendo o campo de deslocamentos chamado de restritivo. Assim, se o número de graus de liberdade do campo restritivo for maior que o do campo primário o sistema resolvente será numericamente instável, levando a primeira hipótese à inviabilidade. 
Formulações não convencionais em elementos finitos

Continuidade em forma fraca

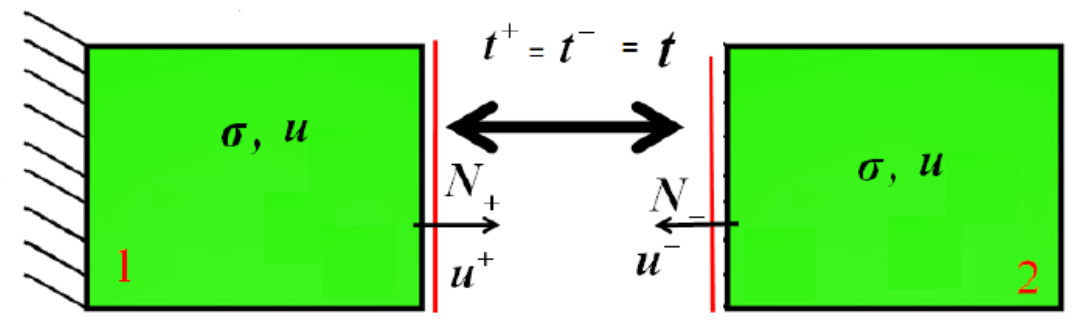

Reciprocidade em forma fraca

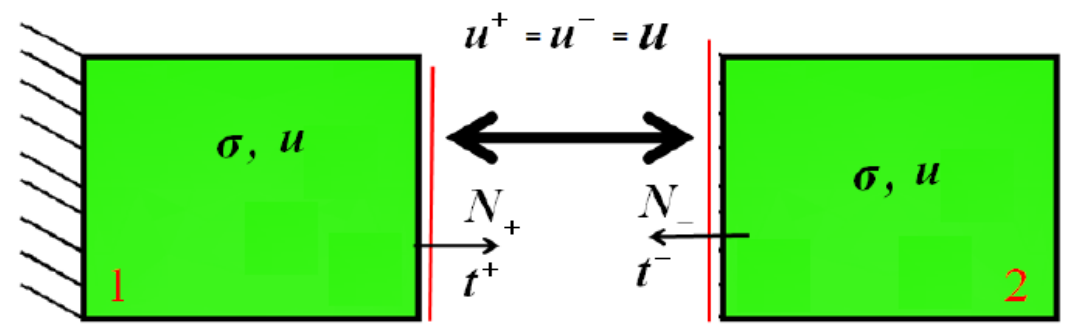

$\mathrm{Na}$ segunda hipótese, onde se admitiu a continuidade de deslocamentos entre elementos, os graus de liberdade de tensão se mantiveram, sem acréscimos, e houve condensação dos campos de deslocamento. Assim esta hipótese obedece à condição de estabilidade citada anteriormente, sendo viável à implementação. 
Formulações Híbridas e Mistas para o Método dos Elementos Finitos Generalizados: aplicação à Mecânica do Dano 
Métodos sem malha

\section{Métodos sem malha}

A motivação principal para o desenvolvimento dos métodos sem malha foi superar alguns inconvenientes apresentados pelo MEF, na sua formulação em deslocamentos. Torres (2003) pontua sobre algumas limitações do MEF como motivação para o desenvolvimento dos métodos sem malha. Dentre estas se destacam a geração de malha que a depender do problema é realizada com grande custo computacional, particularmente quando o procedimento $h$-adaptativo numa certa região do problema demanda uma nova malha de elementos. Outra característica importante é que o MEF clássico não favorece a resolução de problemas no âmbito da mecânica da fratura.

Nos métodos sem malha a solução aproximada do PVC é construída a partir de um conjunto de aproximações locais atreladas a pontos nodais dispersos no domínio sem conectividade pré-estabelecida, não existindo assim, uma malha de elementos finitos.

As funções de forma da maioria dos métodos sem malha são obtidas a partir de variantes do método dos mínimos quadrados, aplicado com o objetivo de aproximar a solução exata mediante um critério de minimização de distância relativa, conforme Proença (2010). Em termos gerais, os elementos fundamentais para construção da aproximação local de determinado ponto do domínio são: uma base de funções, geralmente polinomiais, funções de ponderação e uma região de influência (nuvem).

\subsection{Método das Nuvens $h p$}

Em Duarte (1996), foi apresentada uma nova classe de funções de forma, denominada de família de Nuvens- $h p$. Admite-se, neste método, que cada nuvem constitui o suporte para uma função de forma $\varphi_{i}$ associada. Entendendo-se por suporte a região na qual a função de forma apresenta valores não nulos. 


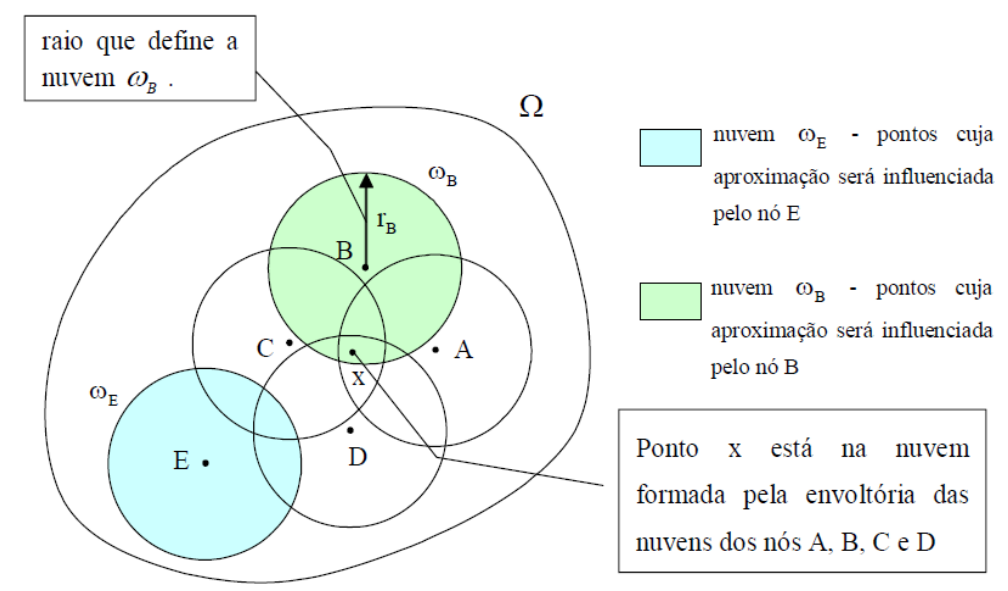

Figura 4.1 - Conjunto de nuvens cobrindo um domínio $\Omega$. Fonte: Proença (2010).

A denominação de $h p$ do Método das Nuvens é devida à sua grande versatilidade para combinar os enriquecimentos do tipo $h$, ao introduzir novos pontos nodais ao domínio, com os do tipo $p$, ao explorar funções de grau superior introduzindo novos parâmetros nodais a elas associados.

Mas a característica mais peculiar do método é o enriquecimento da aproximação, obtido multiplicando-se a base de funções proposta inicialmente por novos monômios ou outro tipo de função, sem a necessidade de novos pontos nodais na discretização original.

A única exigência para que se possa realizar o enriquecimento sobre as funções de forma originais do Método das Nuvens $h p$, é que estas constituam uma Partição da Unidade (PU). Mas essa característica é intrínseca as funções de forma do Método das Nuvens $h p$, pois estas são obtidas a partir das funções aproximadoras do Método dos Mínimos Quadrados Móveis (MMQM) e que constituem uma PU. A exigência mencionada é importante para garantir continuidade da aproximação global resultante de combinação das aproximações locais.

\subsection{Método dos Elementos Finitos Generalizados}

O Método dos Elementos Finitos Generalizados (MEFG) pode ser entendido como uma combinação da forma clássica do MEF com técnicas dos métodos sem malha, especificamente a estratégia de enriquecimento da aproximação adotada no Método das Nuvens $h p$. Assim, o MEFG pode ser inserido entre os métodos sem dependência de rede (Mesh-Free), já que a rede composta por elementos (malhas) e nós não é empregada no sentido clássico, mas sim para definir as nuvens locais e emprestar dos elementos as funções de forma que constituem a PU. 
Métodos sem malha

O MEFG é apresentado como uma forma de obter boas condições de aproximação local (no interior da nuvem), sendo que a PU dos elementos permite combinar as aproximações locais assegurando a conformidade da aproximação global. Além disso, entre outras características, a continuidade da aproximação global possui grau atrelado ao grau de continuidade da PU, as condições de contorno são facilmente impostas, como no MEF, e o método mostra-se robusto mesmo sob forte distorção da rede (resultante do fato que o enriquecimento é feito sobre as coordenadas espaciais).

Vale salientar que há um paralelo entre as versões $h$ e $p$ do MEF e o MEFG. Se todo o espaço das aproximações locais é ampliado e os suportes das funções partição de unidade são mantidos, tem-se uma situação equivalente ao refinamento $p$ do MEF, entretanto sem a introdução de nós adicionais, como nos meios dos lados, por exemplo. Por outro lado, se o espaço das aproximações locais é mantido e a área de influência de cada suporte reduzida, tem-se um processo semelhante ao MEF com refinamento $h$.

No Método das Nuvens $h p$, a nuvem $\left(\omega_{\alpha}\right)$, ou região de definição da função de aproximação local, é determinada, geralmente, por uma região circular em torno de um nó. No MEFG, aproveitando-se a rede de elementos finitos do MEF, a nuvem é formada pelo conjunto de elementos que têm um nó como vértice comum. Na Figura 4.2, procura-se ilustrar esse conceito.

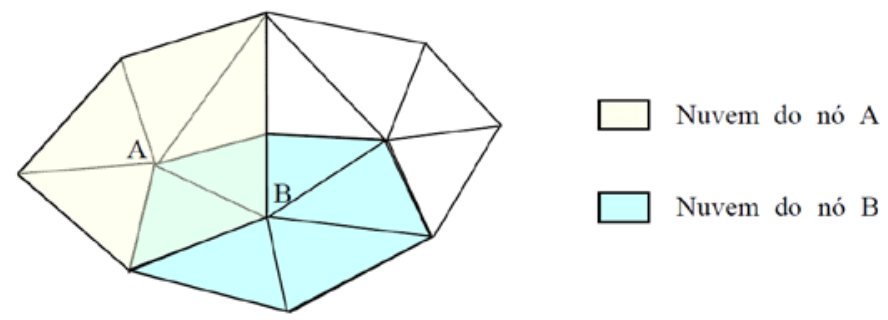

Figura 4.2 - Nuvens no MEFG. Fonte: Proença (2010).

Em cada nuvem define-se uma aproximação básica $\varphi_{\alpha}$ reunindo-se as funções de interpolação de cada elemento atreladas ao nó comum. As aproximações básicas das nuvens são tais que constituem uma partição da unidade (PU). Com isso, num ponto $x$ coberto, por exemplo, pelas nuvens $\alpha$ e $\alpha+1$, tem-se que: $\varphi_{\alpha+1}(x)+\varphi_{\alpha}(x)=1$.

O MEFG propõe, também, a construção de funções de forma a serem usadas nas aproximações dos campos de interesse para determinado problema, em cada nuvem $\alpha$. Tais funções de forma são definidas pelo produto entre a PU e funções de enriquecimento $\left(L_{i \alpha}\right)$. As funções de enriquecimento têm por objetivo melhorar a qualidade da aproximação local e 
podem ter características especiais. Considerando-se todas as nuvens, a família de funções de forma do MEFG pode ser indicada como:

$F_{N}=\left\{\Phi_{\alpha}^{i}\left(=\varphi_{\alpha} L_{i \alpha}\right), \alpha=1, \ldots, N ; i \in I(\alpha)\right\}$

onde não há soma sobre $\alpha$ e vale a seguinte notação:

$\varphi_{\alpha}$ : funções que compõem a PU, definidas nos elementos que formam a nuvem $\alpha \mathrm{e}$ atreladas ao nó comum, base da nuvem;

$I(\alpha)$ : contador para o número de funções de enriquecimento adicionadas em cada nóbase (este número pode ser variável de nuvem para nuvem);

$L_{i \alpha}$ : base de funções de enriquecimento adotadas (e que contém a unidade para $i=1)$.

Como exemplificação da relação anterior, para a nuvem $\alpha$ as funções de forma podem ser reunidas no vetor:

$\beth_{\alpha}=\left\{\Phi_{\alpha}^{1}, \Phi_{\alpha}^{2}, \Phi_{\alpha}^{3}, \ldots, \Phi_{\alpha}^{I(\alpha)}\right\}$

$\beth_{\alpha}=\{\underbrace{\varphi_{\alpha} 1}_{\Phi_{\alpha}^{1}}, \underbrace{\varphi_{\alpha} L_{2(\alpha)}}_{\Phi_{\alpha}^{2}}, \varphi_{\alpha} L_{3(\alpha)}, \ldots, \varphi_{\alpha} L_{I(\alpha)}\}$

Uma propriedade importante decorrente do uso da PU é que se o conjunto $L_{i(\alpha)}$ for o mesmo para todas as nuvens, isto é: $L_{i(\alpha)}=L_{i}$, então combinações lineares das funções de forma do MEFG podem reproduzir as funções locais de enriquecimento. Para ilustrar esse aspecto, considere-se a seguinte combinação de funções de forma:

$\Phi_{\alpha}^{2}+\Phi_{\alpha+1}^{2}=\varphi_{\alpha} L_{2(\alpha)}+\varphi_{\alpha+1} L_{2(\alpha+1)}=\varphi_{\alpha} L_{2}+\varphi_{\alpha+1} L_{2}=\overbrace{\left(\varphi_{\alpha}+\varphi_{\alpha+1}\right)}^{1} L_{2}=L_{2}$

De um modo mais geral, sendo $n_{e}$ o número de nuvens enriquecidas, pode-se estender a conclusão anterior para todas as funções locais de enriquecimento: 
$\sum_{\alpha=1}^{n_{e}} \Phi_{\alpha}^{i}=L_{i}$

Tendo-se em vista as funções de forma do MEFG, aproximações para as soluções de campos de interesse podem ser construídas do seguinte modo:

$\tilde{u}=\sum_{j=1}^{N} \Phi_{j}^{1} u_{j}+\sum_{\alpha=1}^{n_{e}} \sum_{i=2}^{I(\alpha)} \Phi_{\alpha}^{i} b_{\alpha i}$

Onde: $\Phi_{j}^{1}=\varphi_{j} 1, u_{j}$ são parâmetros associados aos graus de liberdade nodais usuais de elementos finitos e $b_{\alpha i}$ são parâmetros nodais adicionais introduzidos pelo enriquecimento. Observa-se, novamente, que a PU proporciona a compatibilização entre todas as aproximações locais, gerando uma aproximação global com grau de continuidade definido pela própria PU.

$\mathrm{Na}$ Figura 4.3 ilustra-se o processo de enriquecimento da partição da unidade preservando a característica de suporte compacto de aproximação resultante.

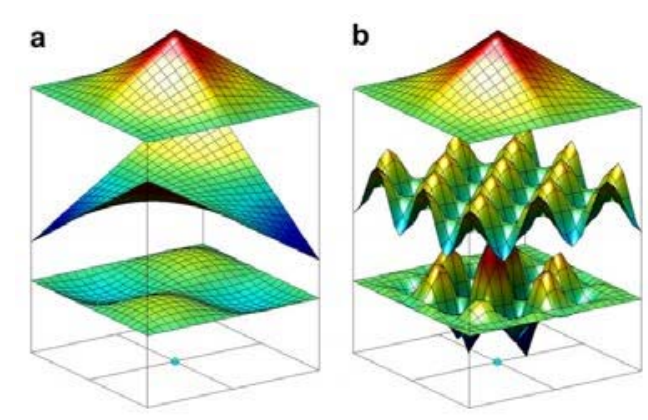

Figura 4.3 - Procedimento de enriquecimento sobre um ponto: resultado (inferior) da multiplicação entre a função de forma (superior) com uma função enriquecedora (centro) polinomial (a) e não polinomial (b). Fonte: Duarte e Kim (2008). 
Formulações Híbridas e Mistas para o Método dos Elementos Finitos Generalizados: aplicação à Mecânica do Dano 


\section{Princípios básicos da Mecânica do Dano}

\subsection{Considerações iniciais}

O dano se manifesta fisicamente em diferentes escalas a partir da formação e desenvolvimento de microvazios ou microfissuras. Na Mecânica do Contínuo estas descontinuidades podem ser consideradas mediante uma porção de meio definida naquilo que se denomina por mesoescala, grande o suficiente para que os defeitos possam ser considerados suficientemente difusos, mas ao mesmo tempo muito pequena em relação à escala do meio (macroescala) (Figura 5.1).

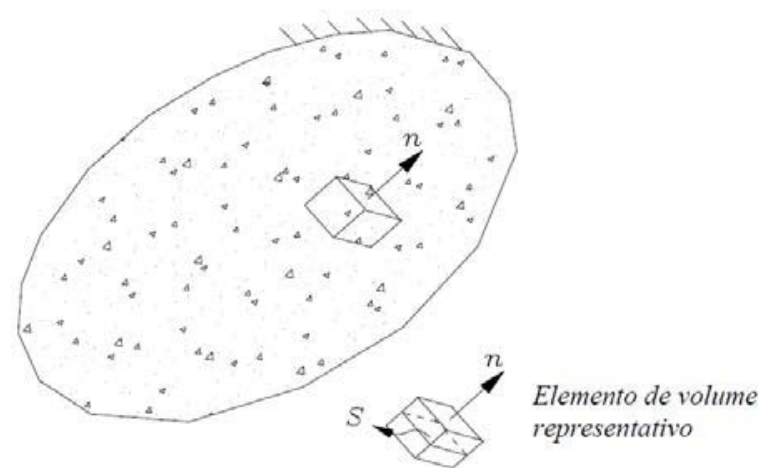

Figura 5.1 - Elemento de volume representativo. Fonte: Proença (2008).

Ao dano no elemento de volume representativo (EVR) pode-se associar uma variável representativa de natureza escalar ou tensorial a depender do grau de complexidade que se deseja dar ao modelo. Neste trabalho, considera-se apenas o caso de dano isotrópico (preserva-se a isotropia inicial mesmo havendo evolução do dano), sendo sua variável escalar representativa, definida conforme a eq.(5.1):

$D=\frac{\delta S_{D}}{\delta S}=\frac{\delta S-\delta \tilde{S}}{\delta S}$

onde $\delta S$ é a área bruta de uma seção transversal do EVR, conforme indica a Figura 5.1, $\delta S_{D}$ a área de cavidades e $\delta \tilde{S}$ a área efetiva da mesma tensão. Na eq. (5.1) fica explícito que a definição aplica-se para qualquer que seja a seção considerada, daí a natureza escalar da variável de dano. A definição de área efetiva resistente permite introduzir o conceito de tensão 
efetiva, mediante a equivalência entre resultantes de tensão na seção, conforme indica a eq.(5.2):

$\sigma \delta S=\tilde{\sigma} \delta \tilde{S} \Rightarrow \tilde{\sigma}=\frac{\sigma}{1-D}$

sendo $\tilde{\sigma}$ a tensão efetiva num ponto qualquer do EVR.

O conceito de tensão efetiva associado a princípio do estado local permite definir uma relação constitutiva para o EVR danificado. Assim, de maior interesse para este trabalho, admitindo-se que o meio danificado preserve a resposta elástica, numa porção íntegra ou resistente do meio atua a tensão efetiva e a deformação é puramente elástica.

Assim sendo, considerando-se por simplificação um caso unidimensional, vale a seguinte relação constitutiva: $\tilde{\sigma}=E \varepsilon$. Com a eq.(5.2) obtém-se a seguinte relação constitutiva que reflete o acoplamento entre o dano e a deformação:

$\sigma=(1-D) E \varepsilon$

Esta forma de penalização da lei de Hooke é comumente utilizada para meios isotrópicos, com dano escalar devendo ser considerados os tensores de tensão deformação no caso geral de solicitação multiaxial.

\subsection{Elementos da termodinâmica dos processos irreversíveis}

As considerações feitas neste item estão fortemente baseadas no texto de Lemaitre e Desmorat (2005), na qual um desenvolvimento mais completo pode ser encontrado. Em resumo, a termodinâmica dos processos irreversíveis permite a modelagem do comportamento de diversos materiais em três passos. O primeiro passo consiste na definição das variáveis de estado representativas dos mecanismos de interesse envolvidos. $\mathrm{O}$ segundo passo consiste na definição do potencial de estado, do qual derivam as chamadas leis de estado e as variáveis associadas às variáveis de estado. Por fim, o terceiro passo consiste na definição de um potencial de dissipação do qual derivam as leis de evolução das variáveis de estado associadas a processos dissipativos. 
Na Tabela 5.1 exemplificam-se diferentes variáveis de estado, cuja natureza pode ser escalar ou tensorial, escolhidas de acordo com diferentes mecanismos físicos de deformação e degradação do material.

Tabela 5.1 - Variáveis de estado e associadas. Fonte: Lemaitre e Desmorat (2005).

\begin{tabular}{|c|c|c|c|c|}
\hline \multirow{2}{*}{ Mecanismos } & \multirow{2}{*}{ Tipo } & \multicolumn{2}{|c|}{ Variáveis de estado } & \multirow{2}{*}{$\begin{array}{l}\text { Variáveis } \\
\text { associadas }\end{array}$} \\
\hline & & Observáveis & Internas & \\
\hline Termoelasticidade & Tensorial & $\varepsilon$ & & $\boldsymbol{\sigma}$ \\
\hline Entropia & Escalar & $T$ & & $S$ \\
\hline Plasticidade & Tensorial & & $\varepsilon^{p}$ & $-\sigma$ \\
\hline Encruamento isótropo & Escalar & & $r$ & $R$ \\
\hline Encruamento cinemático & Tensorial & & $\alpha$ & $\boldsymbol{X}$ \\
\hline \multirow{2}{*}{ Dano } & Escalar (isotrópico) & & $D$ & $-Y$ \\
\hline & Tensorial (anisotrópico) & & $D$ & $-Y$ \\
\hline
\end{tabular}

A energia livre específica de Helmholtz $(\psi)$ é uma função de todas as variáveis de estado e define um potencial de estado do material. De acordo com Lemaitre e Desmorat (2005), alguns resultados qualitativos experimentais sobre a possibilidade de acoplamentos mostram que o potencial de estado $\left[\psi=\left(\boldsymbol{\varepsilon}^{\boldsymbol{e}}, \boldsymbol{D}\right.\right.$ ou $\left.\left.D, r, \boldsymbol{\alpha}, T\right)\right]$ pode ser expresso pela soma de contribuições ditas elástica $\left(\psi_{e}\right)$, plástica $\left(\psi_{p}\right)$, associadas ao encruamento e térmica $\left(\psi_{T}\right)$. Outro potencial que pode ser mais conveniente considerar é o potencial da energia específica livre de Gibbs $\left(\psi^{*}\right)$, que se relaciona com a energia livre de Helmholtz na seguinte forma:

$\psi^{*}=\psi_{e}^{*}+\frac{1}{\rho} \sigma_{i j} \varepsilon_{i j}^{p}-\psi_{p}-\psi_{T}$

onde $\rho$ é a densidade. A contribuição elástica $\psi_{e}^{*}$ inclui a variável de dano por meio do conceito de tensão efetiva para modelar o acoplamento experimentalmente observado entre a elasticidade e o dano. Assim, as relações constitutivas em deformação de um material elástico danificado são análogas às relações de um material elástico não danificado, exceto que a tensão nominal é substituída pela tensão efetiva. Por sua vez a parcela $\psi_{p}$ representa a contribuição do encruamento plástico, dada por:

$\psi_{p}=\frac{1}{\rho}\left(\int_{0}^{r} \Lambda(r) d r+\frac{1}{3} C \alpha_{i j} \alpha_{i j}\right)$ 
sendo $r$ e $\boldsymbol{\alpha}$ variáveis conceitualmente definidas na Tabela 5.1, e tratando-se $\psi_{p}$, a rigor, de uma quantidade de energia armazenada internamente no EVR não recuperável num processo de descarregamento. $\mathrm{O}$ parâmetro $C$ do material está relacionado à parte linear do encruamento cinemático. Por fim, na eq.(5.5) a contribuição $\psi_{T}$ é uma função apenas da temperatura, inicialmente definindo o calor específico do material.

Com isso, as leis de estado podem ser derivadas do potencial de estado descrito pela eq.(5.5):

$$
\begin{gathered}
\varepsilon_{i j}=\rho \frac{\partial \psi^{*}}{\partial \sigma_{i j}}=\rho \frac{\partial \psi_{e}^{*}}{\partial \sigma_{i j}}+\varepsilon_{i j}^{p} \\
s=\frac{\partial \psi^{*}}{\partial T}
\end{gathered}
$$

As outras derivadas apresentadas pela eq.(5.7) definem as variáveis associadas complementares.

$$
\begin{gathered}
R=-\rho \frac{\partial \psi^{*}}{\partial r} \\
X_{i j}=-\rho \frac{\partial \psi^{*}}{\partial \alpha_{i j}} \\
-Y=-\rho \frac{\partial \psi^{*}}{\partial D} \quad\left\{-Y_{i j}=-\rho \frac{\partial \psi^{*}}{\partial D_{i j}}\right\}
\end{gathered}
$$

O segundo princípio da termodinâmica, descrito pela inequação de Clausius-Duhem e associado à energia dissipada, exprime o caráter irreversível dos processos internos:

$$
\sigma_{i j} \dot{\varepsilon}_{i j}^{p}-\underbrace{\left(R \dot{r}+X_{i j} \dot{\alpha}_{i j}\right)}_{\dot{\omega}_{s}}+Y_{i j} \dot{D}_{i j} \geq \frac{q_{i} T_{, i}}{T}
$$

sendo a parcela $q_{i} T_{, i} / T$ uma quantidade de calor associada a processos puramente térmicos, uma interpretação que se pode dar à eq.(5.8) é que a quantidade correspondente à soma da dissipação plástica $\left(\sigma_{i j} \dot{\varepsilon}_{i j}^{p}\right)$ e da dissipação devido ao dano $\left(Y_{i j} \dot{D}_{i j}\right)$ descontada a taxa de densidade de energia armazenada no encruamento $\left(\dot{\omega}_{s}\right)$, representa uma quantidade de energia transformada em calor, irreversível ou dissipada. 
Ainda tendo como referência Lemaitre e Desmorat (2005), as leis cinéticas que governam a evolução das variáveis internas são derivadas de um potencial dito de dissipação $F(\sigma, R, X, Y)$, considerando-se o caso isotérmico, expresso por uma função que deve ser convexa nas variáveis associadas, para assegurar o cumprimento do segundo princípio da termodinâmica. De acordo com experimentos qualitativos que indicam possibilidades de acoplamento de efeitos, para supor tal potencial consideram-se a função critério de plastificação $(f)$, o termo de encruamento cinemático não linear $\left(F_{X}\right)$ e o potencial do dano $\left(F_{D}\right)$. Segue que o potencial de dissipação pode ser dado pela soma: $F=f+F_{X}+F_{D}$. Observando que o critério de plastificação $f$ leva em conta o encruamento isótropo, as leis de evolução das variáveis internas resultam de:

$$
\begin{gathered}
\dot{\varepsilon}_{i j}^{p}=-\dot{\lambda} \frac{\partial F}{\partial\left(-\sigma_{i j}\right)}=\dot{\lambda} \frac{\partial F}{\partial \sigma_{i j}} \\
\dot{r}=-\dot{\lambda} \frac{\partial F}{\partial R} \\
\dot{\alpha}_{i j}=-\dot{\lambda} \frac{\partial F}{\partial X_{i j}} \\
\dot{D}=-\dot{\lambda} \frac{\partial F}{\partial(-Y)}=\dot{\lambda} \frac{\partial F}{\partial Y} \quad\left\{\dot{D}_{i j}=-\dot{\lambda} \frac{\partial F}{\partial\left(-Y_{i j}\right)}=\dot{\lambda} \frac{\partial F}{\partial Y_{i j}}\right\}
\end{gathered}
$$

Nas relações anteriores, incorpora-se um multiplicador $\dot{\lambda}$ de modo a generalizar os conceitos de multiplicador plástico e normalidade típicas dos modelos elasto-(visco-)plásticas.

Nesse sentido, para a descrição completa da elasto-(visco-)plasticidade acoplada com dano, acrescenta-se, também, a definição de taxa de deformação plástica acumulada $(\dot{p})$ que no caso particular do critério de von Mises é dada como:

$\dot{p}=\sqrt{\frac{2}{3} \dot{\varepsilon}_{i j}^{p} \dot{\varepsilon}_{i j}^{p}}$

Entretanto, neste trabalho considera-se apenas o potencial de estado restrito à elasticidade isotrópica linear e dano escalar, definido como:

$\rho \psi_{e}^{*}=\frac{1+v}{2 E} \frac{\sigma_{i j} \sigma_{i j}}{(1-D)}-\frac{v}{2 E} \frac{\sigma_{k k}^{2}}{(1-D)}$ 
onde $E$ é o modulo de elasticidade, $v$ o coeficiente de Poisson e $\sigma_{k k}$ o traço do tensor de tensões (soma das componentes de tensão normal). Assim, a deformação elástica é dada mediante suas componentes pela eq.(5.12).

$\varepsilon_{i j}^{e}=\rho \frac{\partial \psi_{e}^{*}}{\partial \sigma_{i j}}=\frac{1+v}{E} \frac{\sigma_{i j}}{(1-D)}-\frac{v}{E} \frac{\sigma_{k k}}{(1-D)} \delta_{i j}$

Nota-se que $\sigma^{\sigma_{i j}} /(1-D)$ representa a tensão efetiva. A variável associada ao dano escalar $(Y)$ tem a interpretação de densidade de energia liberada, também derivada do potencial de estado:

$Y=\rho \frac{\partial \psi_{e}^{*}}{\partial D}=\frac{\sigma_{e q}^{2} R_{v}}{2 E(1-D)^{2}}$

Na relação anterior introduz-se $R_{v}$ a função de triaxialidade, dada por:

$R_{v}=\frac{2}{3}(1+v)+3(1-2 v)\left(\frac{\sigma_{H}}{\sigma_{e q}}\right)^{2}$

onde $\sigma_{H}=\sigma_{k k} / 3$ é a tensão hidrostática, $\sigma_{e q}$ é a tensão equivalente de Mises, denotada por:

$\sigma_{e q}=\left(\frac{3}{2} \sigma_{i j}^{D} \sigma_{i j}^{D}\right)^{1 / 2} \therefore i, j=1,2,3$

sendo $\sigma_{i j}^{D}$ é a parcela desviadora do tensor de tensões.

Um conceito complementar de interesse é o de tensão equivalente de dano $\left(\sigma^{*}\right)$, definido com a tensão uniaxial que permite determinar a mesma quantidade de energia específica, $Y(1-D)$, movimentada no caso multiaxial. Da particularização da eq.(5.13) para o caso uniaxial, segue que:

$\sigma^{*}=\sigma_{e q} R_{v}^{1 / 2}$ 


\subsection{Análise numérica com dano}

O objetivo da análise desacoplada é permitir uma avaliação simplificada do estado de danificação da estrutura, ou componente estrutural, em correspondência a certo nível de solicitação.

Num primeiro nível de análise desacoplada simplificada não se determina qualquer valor para o dano: essencialmente o objetivo é o de detectar o aparecimento de uma fissura. Inicialmente, o critério de ruptura a ser empregado em cada ponto do meio baseia-se na limitação da densidade de energia liberada $(Y)$ para se atingir certo estado de dano, cujo valor crítico é dado por:

$Y_{c}=\frac{\sigma_{\infty}^{2}}{2 E}$

onde $\sigma_{\infty}$ é uma tensão efetiva limite de ruptura. Por outro lado, para $Y$ vale a relação introduzida pela eq.(5.13). Aplicando-se o critério $Y \leq Y_{c}$, chega-se à condição:

$\frac{\sigma_{e q} R_{v}^{1 / 2}}{(1-D)} \leq \sigma_{\infty}$

$\mathrm{Na}$ iminência da ruptura, o dano atinge um valor crítico $\left(D_{c}\right)$, verificando-se então a igualdade:

$\sigma_{e q} R_{v}^{1 / 2}=\left(1-D_{c}\right) \sigma_{\infty}$

Neste ponto, vale ressaltar que apesar de, formalmente, a variável de dano poder atingir um valor unitário, o que corresponderia teoricamente à ruptura local do EVR do material, este limite não é fisicamente plausível, pois observa-se, experimentalmente, que a partir de certo valor, dito crítico, a evolução do dano passa a se dar em regime instável. Neste sentido, diz-se que a representatividade dos modelos desacoplados de dano é limitada por valores de dano no intervalo: $0 \leq D \leq D_{c}$, sendo este valor, a depender do material, contido nos seguintes limites: $0,2 \leq D_{c} \leq 0,5$. Portanto, é possível explorar o conceito de dano crítico para identificar o aparecimento local de uma fissura no material. 
A abordagem simplificada consiste em aplicar diretamente a relação anterior, ignorando, portanto, como evoluiu todo o processo prévio de danificação responsável pelo estado crítico e sua influência sobre a resposta global do elemento estrutural. Em termos de análise numérica, o que se faz é adotar valores para $\sigma_{\infty}$ e $D_{c}$, e estimar em cada ponto de interesse o valor de $\sigma_{e q} R_{v}^{1 / 2}$. Nos pontos aonde o critério é verificado ou violado há indicação de aparecimento de fissura. É possível, ainda, complementar a análise definindo-se uma orientação para a fissura e determinando-se um valor para o tamanho da fissura discreta equivalente. A orientação da fissura pode ser associada, por exemplo, com a direção normal à da tensão principal maior e a relação de interesse para a estimativa do tamanho da fissura é a seguinte:

$\delta \approx \frac{2 E}{\sigma_{\infty}^{2}} \frac{G_{c}}{D_{c}} \approx \frac{G_{c}}{Y_{c} D_{c}}$

onde $G_{C}$ é a energia crítica de fratura, considerada uma propriedade do material.

Um caso particular inserido nessa análise simplificada é o dos materiais puramente frágeis. Neste tipo de material admite-se que a ruptura ocorre sem deformação plástica ou mesmo dano apreciável, e o critério de ruptura se reduz simplesmente à: $\sigma_{e q} R_{v}^{1 / 2}=\sigma_{\infty}$. Evidencia-se claramente, nesta relação, a influência da razão de triaxialidade sobre a ruptura.

Por outro lado, na análise desacoplada com determinação do valor do dano, sua estimativa se realiza numa etapa de pós-processamento de uma análise estrutural convencional elástica ou elasto-plástica. Em particular, é possível mapear os valores de dano determinados, no sentido de avaliar a extensão da região afetada pela deterioração e identificação dos pontos aonde o dano pode atingir valores críticos.

Nos meios elasto-plásticos, um critério adicional é normalmente inserido neste caso e serve para a identificação do início da danificação. A ideia é reproduzir um fato experimentalmente verificado nos metais: a danificação surge após certo nível acumulado de deformação plástica, isto é, o material primeiro encrua e depois fissura. O critério para o aparecimento do dano se baseia numa medida da deformação plástica acumulada, que pode ser determinada a partir da seguinte relação:

$p=\int_{0}^{t} \dot{p} d t$ 
onde $\dot{p}$ é dado pela eq.(5.10). Caso a medida $(p)$ atinja certo valor limite $\left(p_{D}\right)$, a partir daquele instante haverá evolução do dano. Portanto, a relação que define estados sem danificação se exprime como:

$p \leq p_{D}$

onde $p_{D}$ é um valor de referência adotado de acordo com o tipo de material. Uma vez identificado o aparecimento do dano, sua lei geral de evolução é dada por:

$\dot{D}=\frac{Y}{S} \dot{p} H\left(p-p_{D}\right)$

onde $S$ é um novo parâmetro do material, que pode ser identificado experimentalmente, e $H(\cdot)$ é conhecida como função Heaviside, que assume o valor unitário para argumentos positivos e zero para negativos.

No caso particular de carregamento proporcionalmente crescente, uma vez identificado o critério de dano, é possível mostrar que a relação anterior é integrável, fornecendo:

$D=\frac{\sigma_{\infty}^{2} R_{v}}{2 E S}\left(p-p_{D}\right)$

Em resumo, este último tipo de análise simplificada pode se proceder realizando-se uma análise convencional elasto-plástica. Registram-se em cada iteração e em cada ponto de interesse o vetor incremento de deformação plástica e o valor de sua medida acumulada, além da razão de triaxialidade e da tensão equivalente de Mises (úteis também na verificação do critério de ruptura). O pós-processamento percorre o conjunto de valores registrados, determinando-se as intensidades de dano pela eq.(5.24). Uma vez que se calcula o valor do dano, o critério de ruptura, correspondente ao aparecimento local de uma fissura, pode ser aplicado usando sua relação mais completa, dada pela eq.(5.18). 


\subsection{Análise numérica desacoplada simplificada}

Neste trabalho propõe-se uma simplificação da análise desacoplada, a fim de evitar uma análise plástica ou elasto-plástica de natureza incremental-iterativa. Para isto, se verifica o estado de tensões resultantes de uma análise elástica em duas etapas. Na primeira observa-se em quais pontos o limite elástico foi ultrapassado e na segunda calcula-se o valor da variável de dano para estes pontos.

Para avaliar a integridade elástica do material faz-se uso da eq.(5.18), onde a tensão efetiva limite de ruptura $\sigma_{\infty}$ é substituída pela tensão de escoamento $\sigma_{0}$, conforme a seguir:

$\frac{\sigma_{e q} R_{v}^{1 / 2}}{\left(1-D_{0}\right)} \leq \sigma_{0}$

sendo $D_{0}$ um valor de dano inicial $\left(D_{0}=0\right.$ para material íntegro).

Nos pontos onde a relação dada pela eq.(5.25) é violada ocorre danificação do material. Segue daí que se admite que existam pontos que tenham um comportamento intermediário, isto é, ultrapassaram o limite elástico $\sigma_{0}$ mas não atingiram a tensão de ruptura $\sigma_{\infty}:$

$\sigma_{0}<\frac{\sigma_{e q} R_{v}^{1 / 2}}{(1-D)} \leq \sigma_{\infty}$

e pontos em que o limite superior é violado. Na relação anterior $D$ é o valor do dano ocorrido após atingido o limite elástico e seu valor pode ser calculado conforme indicado a seguir, onde $\overline{\bar{\sigma}}$ é uma tensão resultante de danificação:

$D=\frac{\overline{\bar{\sigma}}-\sigma_{0}}{\overline{\bar{\sigma}}} \quad \therefore \overline{\bar{\sigma}}=\frac{\sigma_{e q} R_{v}^{1 / 2}}{\left(1-D_{0}\right)}$

Da relação anterior pode-se ainda obter uma estimativa para o dano crítico do problema, caso hajam pontos em que o limite de $\sigma_{\infty}$ foi ultrapassado:

$D_{c}=\max _{i=1}^{N_{D}}\left\{(D)_{i}\right\}$ 
onde $N_{D}$ é o número de pontos com danificação.

Como critério geral, nos pontos onde o limite superior da eq.(5.26) é violado admite-se a ocorrência de ruptura local do material.

\subsection{Análise simplificada elasto-plástica localmente acoplada com dano}

A análise localmente acoplada permite levar em conta histórias de carregamentos não proporcionais, quando a relação para o cálculo do dano não é diretamente integrável, sendo, porém, restrita aos casos onde o dano se manifesta em pequenas regiões do meio. Esse tipo de análise consiste inicialmente em realizar, considerando todo o domínio do corpo, uma simulação elasto-plástica convencional não linear, atingindo-se certo nível de carregamento em passos de carga e registrando-se, em cada passo, a evolução das deformações e tensões, além do fator de triaxialidade.

No pós-processamento, em cada passo de carregamento, ou de acordo com uma frequência previamente estabelecida, identifica-se a região mais solicitada do meio empregando-se a relação: $\sigma_{e q} R_{v}^{1 / 2}$. Então, com a distribuição de tensões determinada inicialmente com o modelo elasto-plástico convencional verifica-se se o critério de aparecimento de dano, dado pela eq.(5.22), é violado na região. Uma nova análise restrita a região é realizada, integrando-se o modelo elasto-plástico com dano num procedimento de previsão e correção. Nesse procedimento, o dado de entrada é o nível total de deformação no passo registrado na análise convencional. Naturalmente os valores de dano obtidos são acumulados de passo para passo para fins de comparação com o nível de dano crítico.

A simplificação inerente neste procedimento em relação a uma análise totalmente acoplada é que não se leva em conta a influência da nova distribuição de tensões sobre a análise global de equilíbrio, à medida que ocorre danificação. Desta forma, este procedimento proporciona estimativas pessimistas ou conservativas sobre a resistência do elemento estrutural, prevendo a ruptura num nível de tensão inferior ao que realmente pode ser resistido.

\subsection{Análise acoplada com dano}

\subsubsection{Um modelo de dano isótropo com variável de dano escalar}

Neste item descreve-se o modelo de dano isótropo com uma variável escalar, proposto por Comi e Perego (2001) e adotado por Silva (2006) nas simulações totalmente acopladas 
com elementos finitos híbrido-mistos de tensão. Este modelo de dano é baseado na seguinte definição da densidade de energia livre de Helmholtz:

$\psi=\frac{1}{2}(1-D) \boldsymbol{\varepsilon}^{\mathrm{T}} \mathbf{D} \boldsymbol{\varepsilon}+\psi_{\text {in }}(\xi)$

onde as variáveis de estado são a deformação elástica $(\varepsilon)$, a variável de dano $(D)$, a variável interna escalar $(\xi)$ e a função $\psi_{\text {in }}(\xi)$ corresponde à uma densidade de energia anelástica. A variável interna escalar ( $\xi$ ) possui conotação cinemática e permite descrever rearranjos da microestrutura devido ao desenvolvimento de dano, onde o único mecanismo de dissipação considerado neste modelo é o dano. Vale lembrar que D é a matriz constitutiva de rigidez, apresentada no capítulo 2 .

Comi e Perego (2001) propõem a seguinte expressão para a parcela referente à densidade de energia anelástica:

$\psi_{\text {in }}(\xi)=-k(1-\xi) \sum_{i=0}^{n} \frac{n !}{i !} \ln ^{i}\left(\frac{c}{1-\xi}\right)$

onde $n, k$ e $c$ são parâmetros dependentes do material.

Da eq.(5.29), obtém-se as seguintes equações de estado:

$$
\begin{gathered}
\boldsymbol{\sigma}=\frac{\partial \psi}{\partial \boldsymbol{\varepsilon}}=(1-D) \mathbf{D} \boldsymbol{\varepsilon} \\
Y=-\frac{\partial \psi}{\partial D}=\frac{1}{2} \boldsymbol{\varepsilon}^{\mathrm{T}} \mathbf{D} \boldsymbol{\varepsilon} \\
\chi=\frac{\partial \psi}{\partial \xi}=\psi_{\text {in }}{ }^{\prime}(\xi)=k \ln ^{n}\left(\frac{c}{1-\xi}\right)
\end{gathered}
$$

onde as variáveis associadas são a tensão $(\boldsymbol{\sigma})$, a taxa de energia de deformação inelástica $(Y)$ e uma força termodinâmica $(\chi)$.

Por outro lado, o potencial de dissipação é dado por:

$$
f(Y-\chi)=Y-\chi=\frac{1}{2} \boldsymbol{\varepsilon}^{\mathbf{T}} \mathbf{D} \boldsymbol{\varepsilon}-k \ln ^{n}\left(\frac{c}{1-\xi}\right) \leq 0
$$


As leis de evolução das variáveis internas são obtidas a partir do potencial de dissipação dado pela eq.(5.32).

$$
\begin{gathered}
\dot{D}=\frac{\partial f}{\partial Y} \dot{\gamma}=\dot{\gamma} \\
\dot{\xi}=-\frac{\partial f}{\partial \chi} \dot{\gamma}=\dot{\gamma}
\end{gathered}
$$

onde $\gamma$ representa um escalar positivo. Observando a eq.(5.33) imediatamente conclui-se que, para este modelo constitutivo, o valor da variável de dano $(D)$ coincide com o valor da variável interna $(\xi)$ e ainda com o escalar positivo $(\gamma)$. Vale salientar que as condições de carga e descarga de Kuhn-Tucker são: $(\dot{\gamma} \geq 0, f \leq 0, \dot{\gamma} f=0)$.

Diante do exposto, inicialmente avalia-se a função $\psi_{\text {in }}(\xi)$, onde se adotou um concreto com os parâmetros $k=0,00015 \mathrm{MPa}$ e $c=2,72$, também utilizados por Silva (2006), e variou-se o parâmetro $n$ para $0 \leq \xi<1$ (Figura 5.2 e Figura 5.3).

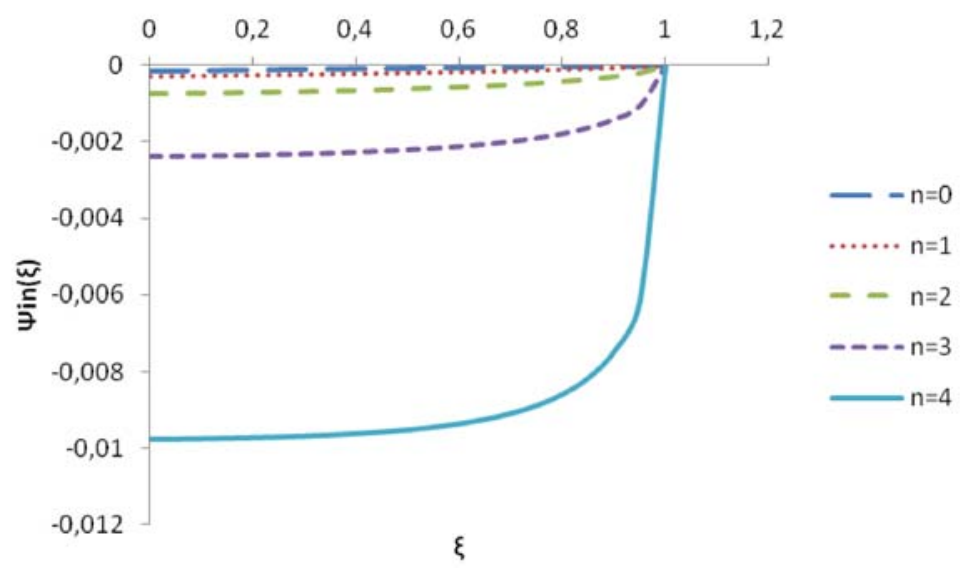

Figura 5.2 - Análise da função $\psi_{i n}(\xi)$ para diferentes valores de $n$. 


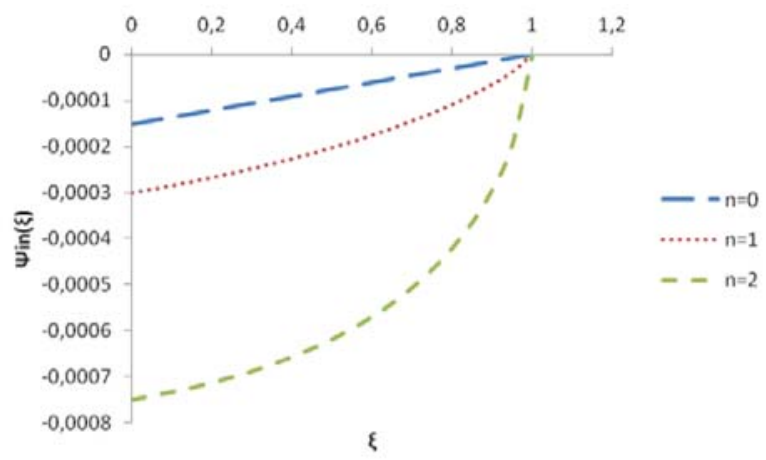

Figura 5.3 - Análise da função $\psi_{i n}(\xi)$ para diferentes valores de $n$ (detalhe).

Quanto maior o valor de $n$ e menor o valor de $\xi$, menor é o valor da função $\psi_{\text {in }}(\xi)$. Como se observa que todas as ordenadas são negativas pode-se afirmar que a parcela anelástica $\left(\psi_{\text {in }}(\xi)\right)$ apresenta maiores valores absolutos para a condição citada $(\uparrow n \downarrow \xi)$. Quando $\xi \rightarrow 1$ tem-se que $\psi_{\text {in }}(\xi) \rightarrow 0$, qualquer que seja o valor de $n$. Entendida a influência do parâmetro $n$ no comportamento da parcela anelástica, pode-se afirmar que a densidade de energia livre de Helmholtz $(\psi)$, previamente apresentada na eq.(5.29), também apresenta menores valores quando do aumento do valor de $n$.

Como é sabido, o potencial de dissipação depende de derivadas parciais da densidade de energia livre de Helmholtz: $f=Y-\chi=(-\psi, D)-\psi, \xi$. Assim, como a variável $Y$ não depende do parâmetro $n$, é avaliada a resposta de $\chi$ quando da variação deste mesmo parâmetro (Figura 5.4 e Figura 5.5).

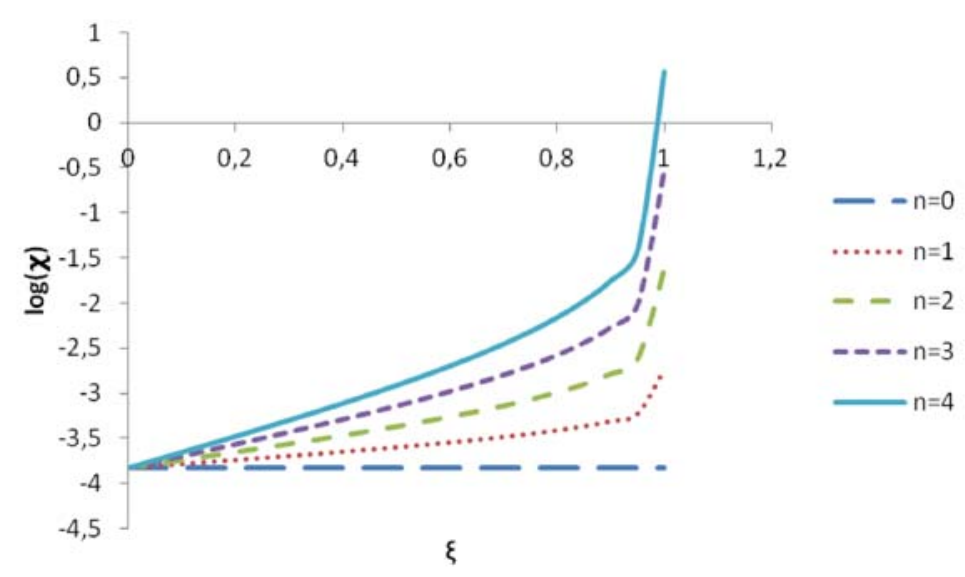

Figura 5.4 - Análise da derivada parcial da função $\psi$ em relação a $\xi .[\chi=\psi, \xi]$. 
Princípios básicos da Mecânica do Dano

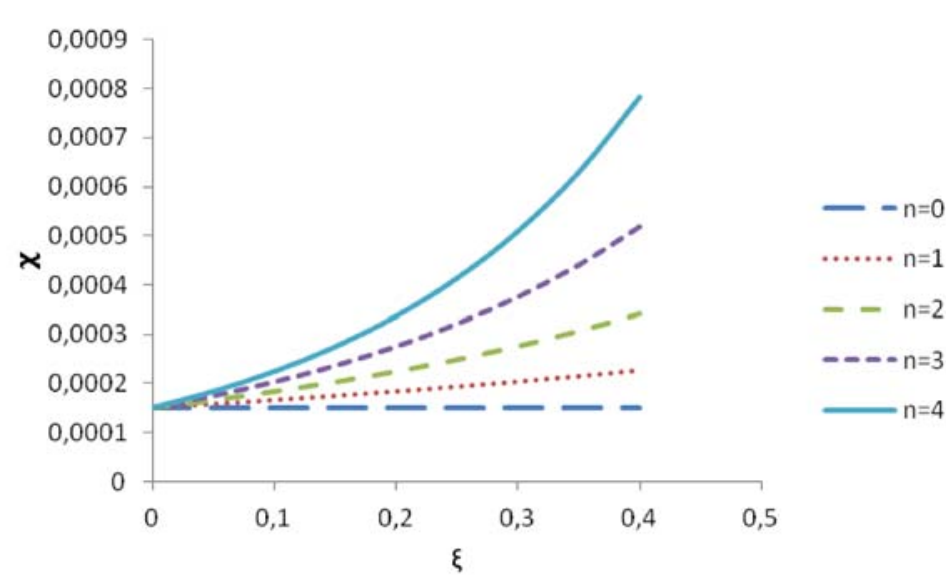

Figura 5.5 - Resposta de $(\chi-\xi)$ para diferentes valores de $n$.

Analisando os gráficos anteriores pode-se afirmar que o valor da força termodinâmica $(\chi)$ tem crescimento mais acelerado quanto maior é o valor de $n$. Este fato influencia diretamente o potencial de dissipação, resultando em diferentes comportamentos do material durante o regime não linear.

Admite-se que o concreto utilizado neste trabalho possui módulo de elasticidade $E=30 \mathrm{GPa}$ e verifica-se seu comportamento não linear para diferentes valores de $n$ (Figura $5.6)$.

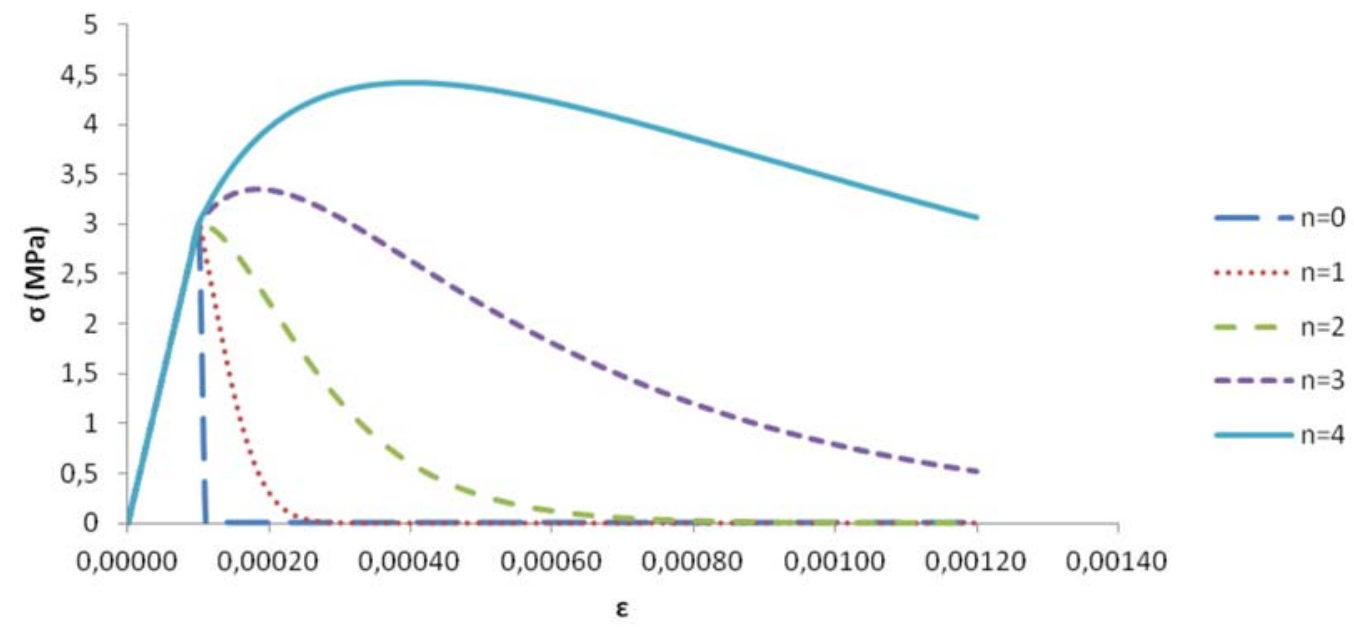

Figura 5.6 - Comportamento unidimensional do modelo constitutivo para diferentes valores de $n$.

Como esperado, observa-se que a variação do parâmetro $n$ influencia diretamente o modelo constitutivo. Para $n=1,2,3,4$ o material apresenta dissipação de energia por encruamento, diferente de quando $n=0$, onde o modelo descreve um modo de ruptura frágil do material. Para $n=1$ e $n=2$ o concreto apresenta encruamento negativo (amolecimento), 
Formulações Híbridas e Mistas para o Método dos Elementos Finitos Generalizados: aplicação à Mecânica do Dano

sendo $n=2$ o parâmetro adotado por Silva (2006). Já para $n=3$ e $n=4$ foi observado encruamento positivo (endurecimento), sendo esta característica de modelo constitutivo adotada neste trabalho, considerando $n=3$. Este comportamento, apesar de improvável para descrever o concreto a tração, é adotado apenas para que seja possível comparar o modelo de dano simplificado, descrito no item 5.4, com a análise acoplada com dano, aqui descrita.

\subsubsection{Modelo não linear}

Neste item descreve-se o modelo não linear com dano adotado, baseado nos trabalhos de Silva (2006) e Arruda (2011). Vale lembrar que os sistemas governativos das formulações Híbrido-Mista e puramente Mista são expressos conforme as eq.(5.34) e eq.(5.35), respectivamente.

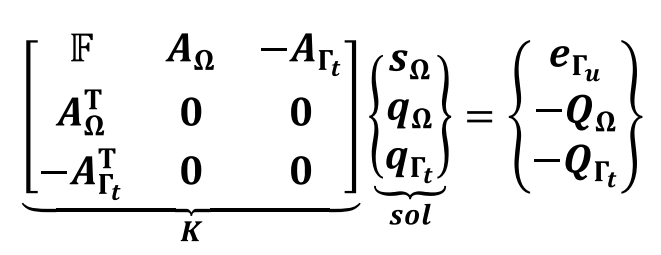

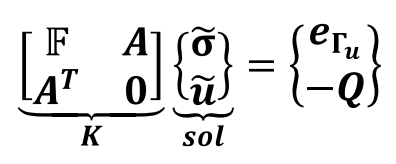

onde $\mathbb{F}$ é a matriz de flexibilidade generalizada, penalizada pela variável escalar de dano $(D)$.

$$
\mathbb{F}=\int_{\Omega} \boldsymbol{S}_{\Omega}^{\mathrm{T}} \tilde{\boldsymbol{f}} \boldsymbol{S}_{\Omega} d \boldsymbol{\Omega} \quad \therefore \tilde{f}=\frac{1}{(1-D)} \boldsymbol{f}
$$

Assim, tem-se que $\mathbb{F}=\boldsymbol{F}$ quando o limite elástico não for ultrapassado.

Num procedimento de análise passo a passo, para um passo de carga arbitrário resolve-se o sistema governativo:

$$
\boldsymbol{K} \Delta \boldsymbol{s o l}=\boldsymbol{R}
$$

onde a matriz $\boldsymbol{K}$ representa a matriz secante ao sistema governativo, $\Delta \boldsymbol{s o l}$ é o vetor solução incremental e $\boldsymbol{R}$ é o vetor de resíduos, cujo cálculo está indicado mais adiante.

Com a variação $\Delta \boldsymbol{s o l}$ encontrada para o passo corrente, atualiza-se o vetor solução: 
$\operatorname{sol}^{(i+1)} \leftarrow \operatorname{sol}^{(i)}+\Delta$ sol

Com o novo vetor solução, procura-se calcular o dano, se ocorrer. Para isto, empregase o potencial de dissipação do meio, reproduzido abaixo:

$f=Y-\chi \leq 0 \quad \therefore\left\{\begin{array}{c}Y=\frac{1}{2} \widetilde{\boldsymbol{\sigma}}^{\mathbf{T}} \boldsymbol{f} \widetilde{\boldsymbol{\sigma}} \\ \chi=k\left[\ln \left(\frac{c}{1-D^{(i)}}\right)\right]^{n}\end{array}\right.$

Quando o valor do potencial desobedece a restrição dada pela eq.(5.39) $(f>0)$, determinam-se novos valores para a variável de dano $(D)$ :

$$
D^{(i+1)}=1-\frac{c}{\exp \left(\sqrt[n]{\frac{Y}{k}}\right)}
$$

Assim, com base nos novos valores de dano atualiza-se a matriz $\boldsymbol{K}$ do sistema governativo e determina-se o vetor de resíduos $\boldsymbol{R}$ :

$\boldsymbol{R}=\boldsymbol{P}-\boldsymbol{K}$ sol $\quad$ onde: $\left\{\begin{array}{l}\boldsymbol{P}=\left\{\begin{array}{c}\boldsymbol{e}_{\boldsymbol{\Gamma}_{u}} \\ -\boldsymbol{Q}_{\Omega} \\ -\boldsymbol{Q}_{\boldsymbol{\Gamma}_{t}}\end{array}\right\} \quad \text { FHMT } \\ \boldsymbol{P}=\left\{\begin{array}{c}\boldsymbol{e}_{\boldsymbol{\Gamma}_{\boldsymbol{u}}} \\ -\boldsymbol{Q}\end{array}\right\} \quad \mathrm{FM}(\mathrm{u}-\sigma)\end{array}\right.$

Com o novo vetor de resíduos é atualizada a quantidade de controle do erro $(\bar{e})$, definida como:

$\bar{e}=\frac{\|R\|}{\bar{C}}$

sendo $\bar{C}$ uma constante de normalização, definida neste trabalho como a norma do primeiro vetor de solução incremental: $\|\Delta \boldsymbol{s o l}\|_{0}$. 
Caso o valor do erro $(\bar{e})$ seja maior que a tolerância admitida, reinicia-se o procedimento a partir da eq.(5.37). 


\section{Resultados}

\subsection{Teste de distorção}

A fim de comparar as formulações em elementos finitos apresentadas neste trabalho considera-se, inicialmente, o teste de distorção, apresentado em Zienkiewicz e Taylor (2000). Este exemplo tem analogia com o problema de uma viga em balanço solicitada por um binário na sua extremidade livre (Figura 6.1). Na discretização empregam-se apenas dois elementos finitos, onde se pretende analisar a qualidade da resposta dada uma distorção $a=$ $\beta \mathrm{L}(0 \leq \beta \leq 1)$. Como se trata de uma análise estritamente ligada à sensibilidade numérica, não são empregadas dimensões para os dados. Considera-se espessura unitária e as seguintes propriedades elásticas: $v=0$ e $E=75$.

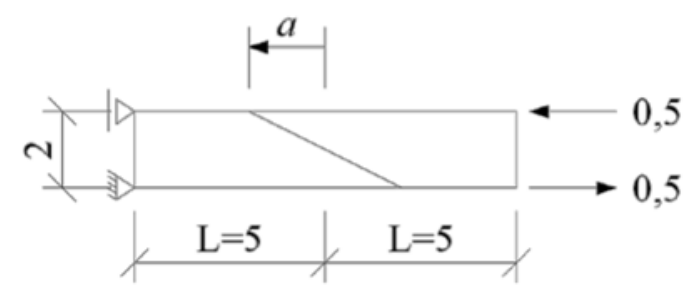

Figura 6.1 - Geometria, carregamento e vinculação de chapa discreta com dois elementos finitos.

Observa-se que a solução de referência para o deslocamento vertical na extremidade livre é igual a 1,00. A notação para o deslocamento na extremidade livre foi escolhida como $v(a)$, para indicar que sua relação com a intensidade de distorção imposta.

Este exemplo foi simulado com a FHMT e FM(u- $\sigma)$, com e sem enriquecimento, além do MEF convencional em deslocamentos e do MEFG (em deslocamentos com enriquecimento). As funções enriquecedoras adotadas, aplicadas em todos os nós livres, foram $L_{2(\alpha)}^{\sigma[\Omega]}=\left\{1, y^{2}\right\}$ e $L_{2(\alpha)}^{\Gamma}=\{1, x\}$ para as tensões de domínio e os deslocamentos de contorno na FHMT, respectivamente, $L_{3(\alpha)}=\{1, x, y\}$ para todos os campos independentes da $\operatorname{FM}(\mathrm{u}-\sigma)$, e $L_{6(\alpha)}=\left\{1, x, y, x y, x^{2}, y^{2}\right\}$ para o MEFG. Os resultados obtidos podem ser avaliados na Figura 6.2. 


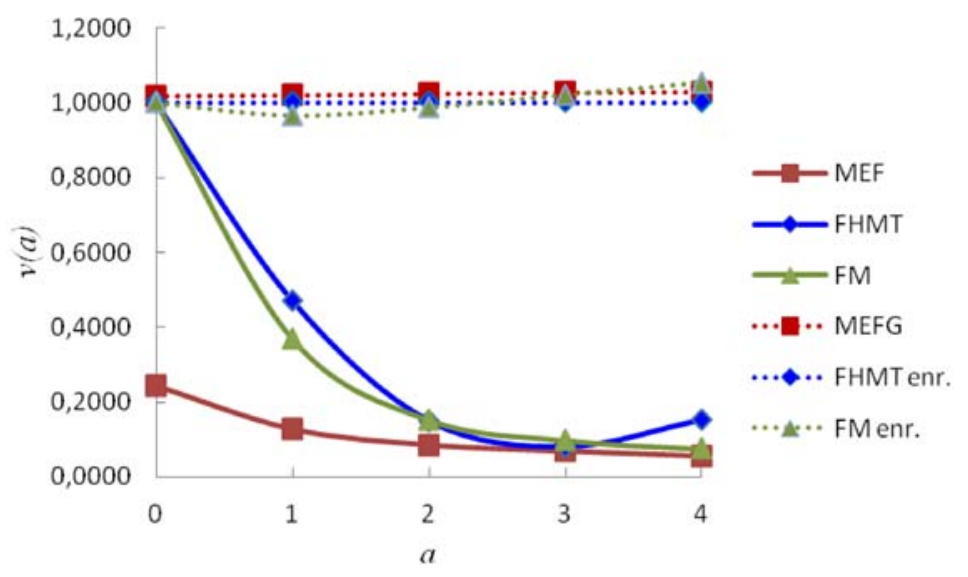

Figura 6.2 - Resultados para o teste de distorção (distorção - deslocamento na extremidade livre).

De acordo com o gráfico apresentado na Figura 6.2, as formulações propostas FHMT e $\operatorname{FM}(\mathrm{u}-\sigma)$ recuperam a solução exata somente quando não há distorção nos elementos. Já o MEF convencional não apresenta bom resultado sequer nesta situação. Entretanto, com o aumento na distorção dos elementos a FHMT e a FM(u- $\sigma)$ perdem precisão. Contudo, a aplicação de funções enriquecedoras tornou as formulações pouco sensíveis à distorção, praticamente recuperando a solução de referência, independente do valor de $a$. Também vale salientar a ótima resposta do MEFG em deslocamentos. Conclui-se que as funções de enriquecimento asseguram maior robustez às formulações numéricas testadas.

\subsection{Chapa tracionada}

Este exemplo trata de uma chapa com espessura unitária, $v=0,3, E=1000 \mathrm{e}$ tracionada por carga $p=10$, parcialmente distribuída nas bordas, conforme apresentado na Figura 6.3. Dada a dupla simetria do problema, o modelo pode ser simplificado conforme indicado na Figura 6.4.

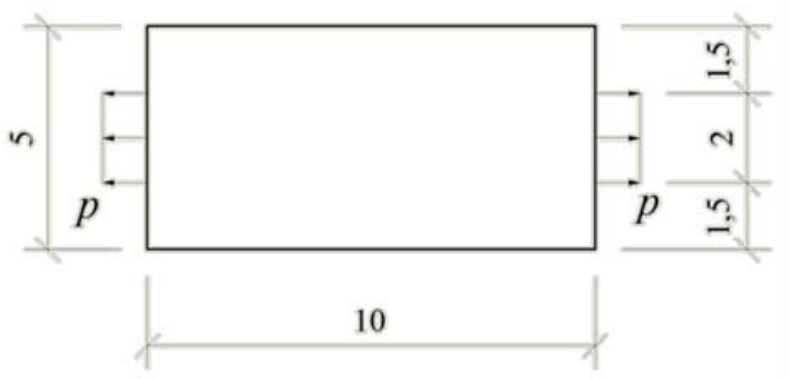

Figura 6.3 - Chapa tracionada. 
Resultados

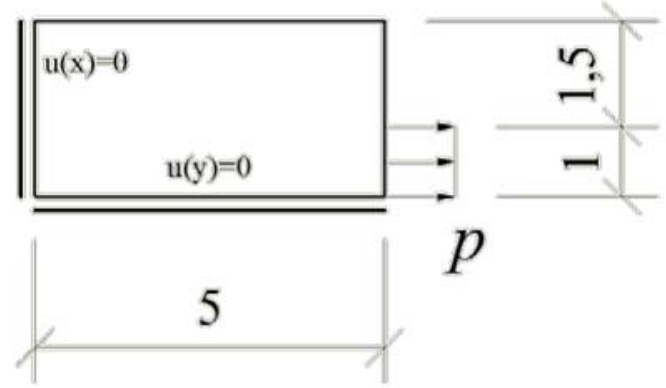

Figura 6.4 - Modelo de análise.

Utilizou-se a ferramenta computacional $\operatorname{ANSYS}^{\circledR}$ (SAS, 2009) para a obtenção de uma solução de referência do problema, com uma discretização de 80x80 elementos finitos do tipo PLANE42 (quadrilateral de quatro nós). Para fins de comparação foi determinada a energia de deformação do problema: $U_{r e f}=0,144$.

Este exemplo foi simulado utilizando-se a FHMT e a $\mathrm{FM}(\mathrm{u}-\sigma)$, com e sem enriquecimento, analisando-se a convergência das respostas em função do aumento do número de graus de liberdade, tanto por refinamento quanto pelo enriquecimento da aproximação. Para o refinamento da discretização redes de 1x1, 2x2, 4x4, 8x8 e 16x16 elementos foram adotadas. As funções de enriquecimento para a FHMT foram: $L_{2(\alpha)}^{\Omega}=$ $\{1, x y\}$ e $L_{2(\alpha)}^{\Gamma}=\{1, x\}$ para os campos de domínio e contorno, respectivamente. Já para a FM(u- $\sigma)$ a função enriquecedora $L_{2(\alpha)}=\{1, x\}$ foi aplicada a ambos os campos independentes.

Diante do exposto, observa-se que a gama de possibilidades de simulação para cada formulação é grande, dado que o número de graus de liberdade depende da discretização e da aplicação das funções enriquecedoras. Entretanto, qualquer que seja a combinação adotada é necessário garantir a estabilidade numérica do problema e, para isto, as formulações precisam ter mais graus de liberdade em tensão do que em deslocamento $\left(n_{\sigma} \geq n_{u}+n_{u_{\Gamma}}\right.$ para a FHMT e $n_{\sigma} \geq n_{u}$ para a $\left.\operatorname{FM}(\mathrm{u}-\sigma)\right)$. Por exemplo, a verificação prévia da condição de estabilidade numérica para a FHMT pode ser vista na Tabela 6.1, e para a FM(u- $\sigma)$ vide Tabela 6.2. 
Formulações Híbridas e Mistas para o Método dos Elementos Finitos Generalizados: aplicação à Mecânica do Dano

Tabela 6.1 - Verificação da estabilidade numérica do modelo para a FHMT.

\begin{tabular}{c|c|c|c|c|c|c|c|c|c|c}
\hline \multicolumn{10}{c}{ Número de graus de liberdade - FHMT } \\
\hline Discretização & \multicolumn{2}{|c|}{$\mathbf{1 x}$} & \multicolumn{2}{c|}{$\mathbf{2 x 2}$} & \multicolumn{2}{|c|}{$\mathbf{4 x 4}$} & \multicolumn{2}{|c|}{$\mathbf{8 x 8}$} & \multicolumn{3}{|c}{$\mathbf{1 6 x 1 6}$} \\
\hline Enriquecimento & $n_{\sigma}$ & $n_{u}+n_{u_{\Gamma}}$ & $n_{\sigma}$ & $n_{u}+n_{u_{\Gamma}}$ & $n_{\sigma}$ & $n_{u}+n_{u_{\Gamma}}$ & $n_{\sigma}$ & $n_{u}+n_{u_{\Gamma}}$ & $n_{\sigma}$ & $n_{u}+n_{u_{\Gamma}}$ \\
\hline Nenhum & 12 & 12 & 27 & 30 & 75 & 90 & 243 & 306 & 867 & 1122 \\
\hline$\sigma[\Omega]$ & 24 & 12 & 54 & 30 & 150 & 90 & 486 & 306 & 1734 & 1122 \\
\hline$\sigma[\Omega], u[\Omega]$ & 24 & 20 & 54 & 48 & 150 & 140 & 486 & 468 & 1734 & $\mathbf{1 7 0 0}$ \\
\hline$\sigma[\Omega], u[\Gamma]$ & 24 & 16 & 54 & 42 & 150 & 130 & 486 & 450 & 1734 & 1666 \\
\hline$\sigma[\Omega], u[\Omega], u[\Gamma]$ & 24 & 24 & 54 & 60 & 150 & 180 & 486 & 612 & 1734 & 2244 \\
\hline
\end{tabular}

Tabela 6.2 - Verificação da estabilidade numérica do modelo para a FM(u- $\sigma)$.

\begin{tabular}{|c|c|c|c|c|c|c|c|c|c|c|}
\hline \multicolumn{11}{|c|}{ Número de graus de liberdade - FM(u- $\sigma)$} \\
\hline \multirow{2}{*}{$\begin{array}{c}\text { Discretização } \\
\text { Enriquecimento }\end{array}$} & \multicolumn{2}{|c|}{$1 \times 1$} & \multicolumn{2}{|c|}{$2 \times 2$} & \multicolumn{2}{|c|}{$4 \times 4$} & \multicolumn{2}{|c|}{$8 \times 8$} & \multicolumn{2}{|c|}{$16 \times 16$} \\
\hline & $n_{\sigma}$ & $n_{u}$ & $n_{\sigma}$ & $n_{u}$ & $n_{\sigma}$ & $n_{u}$ & $n_{\sigma}$ & $n_{u}$ & $n_{\sigma}$ & $n_{u}$ \\
\hline Nenhum & 12 & 04 & 27 & 12 & 75 & 40 & 243 & 144 & 867 & 544 \\
\hline$\sigma$ & 24 & 04 & 54 & 12 & 150 & 40 & 486 & 144 & 1734 & 544 \\
\hline$u$ & 12 & 08 & 27 & 24 & 75 & 80 & 243 & 288 & 867 & 1088 \\
\hline$\sigma, u$ & 24 & 08 & 54 & 24 & 150 & 80 & 486 & 288 & 1734 & 1088 \\
\hline
\end{tabular}

Os campos destacados em cinza mostram as situações onde a condição de estabilidade numérica não é atendida, de modo que os enriquecimentos indicados foram evitados. Entretanto, é importante destacar que se trata de condição necessária, mas não suficiente para estabilidade numérica. De fato, destaca-se na rede de 16x16 elementos da FHMT com enriquecimento em tensões e deslocamentos de domínio exclusivamente onde apesar da condição de estabilidade ter sido atendida, notou-se instabilidade numérica na análise. Com isso, esta simulação foi descartada. Já na $\operatorname{FM}(\mathrm{u}-\sigma)$, destacam-se as análises com enriquecimento apenas em tensão, pois mesmo satisfazendo a condição de estabilidade numérica, a formulação não apresentou bons resultados, mostrando-se, neste caso, sensível ao enriquecimento de apenas um dos campos independentes.

Assim, a Figura 6.5 apresenta a verificação de convergência para a FHMT e a Figura 6.6 a mesma verificação para a $\operatorname{FM}(\mathrm{u}-\sigma)$. 
Resultados

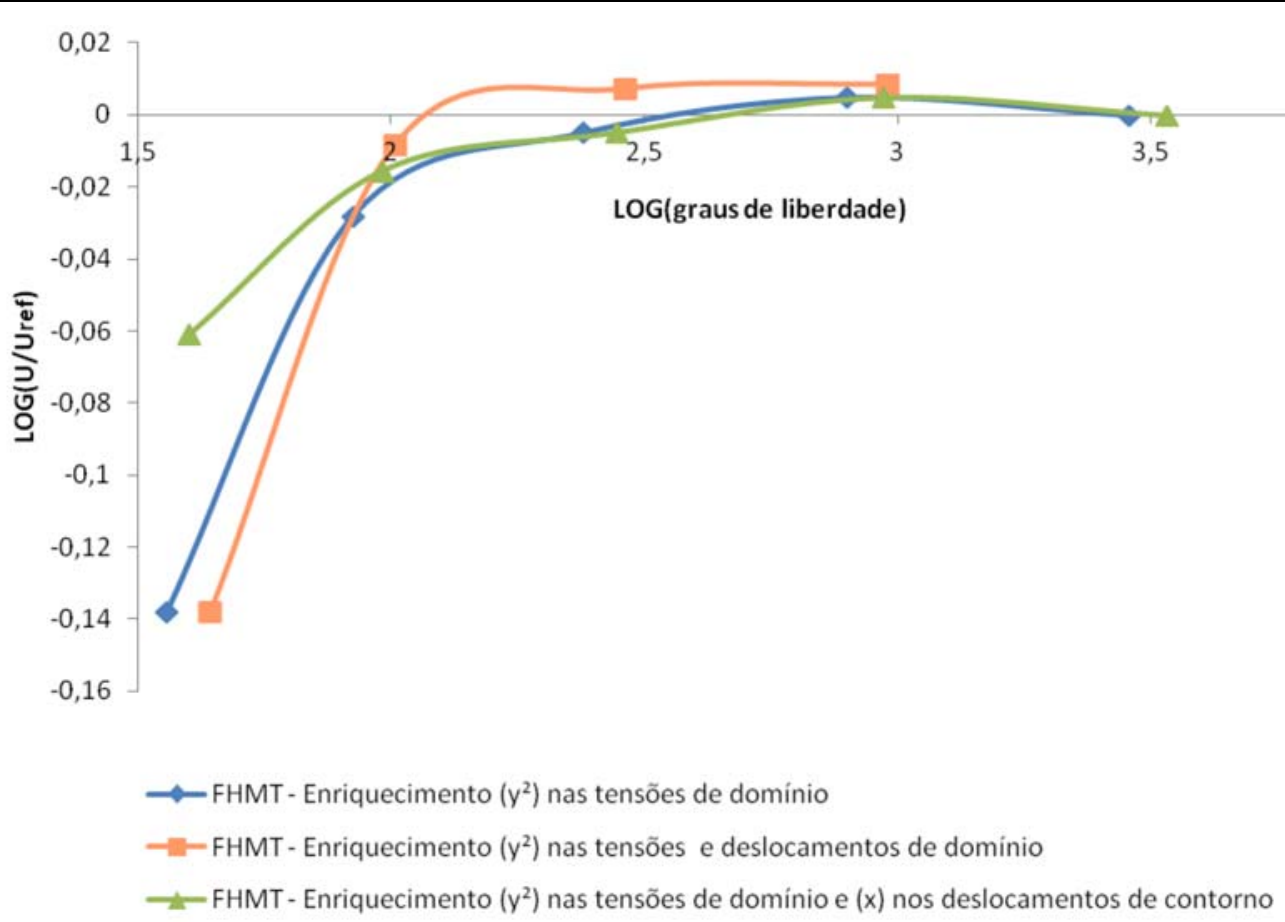

Figura 6.5 - Verificação de convergência da FHMT.

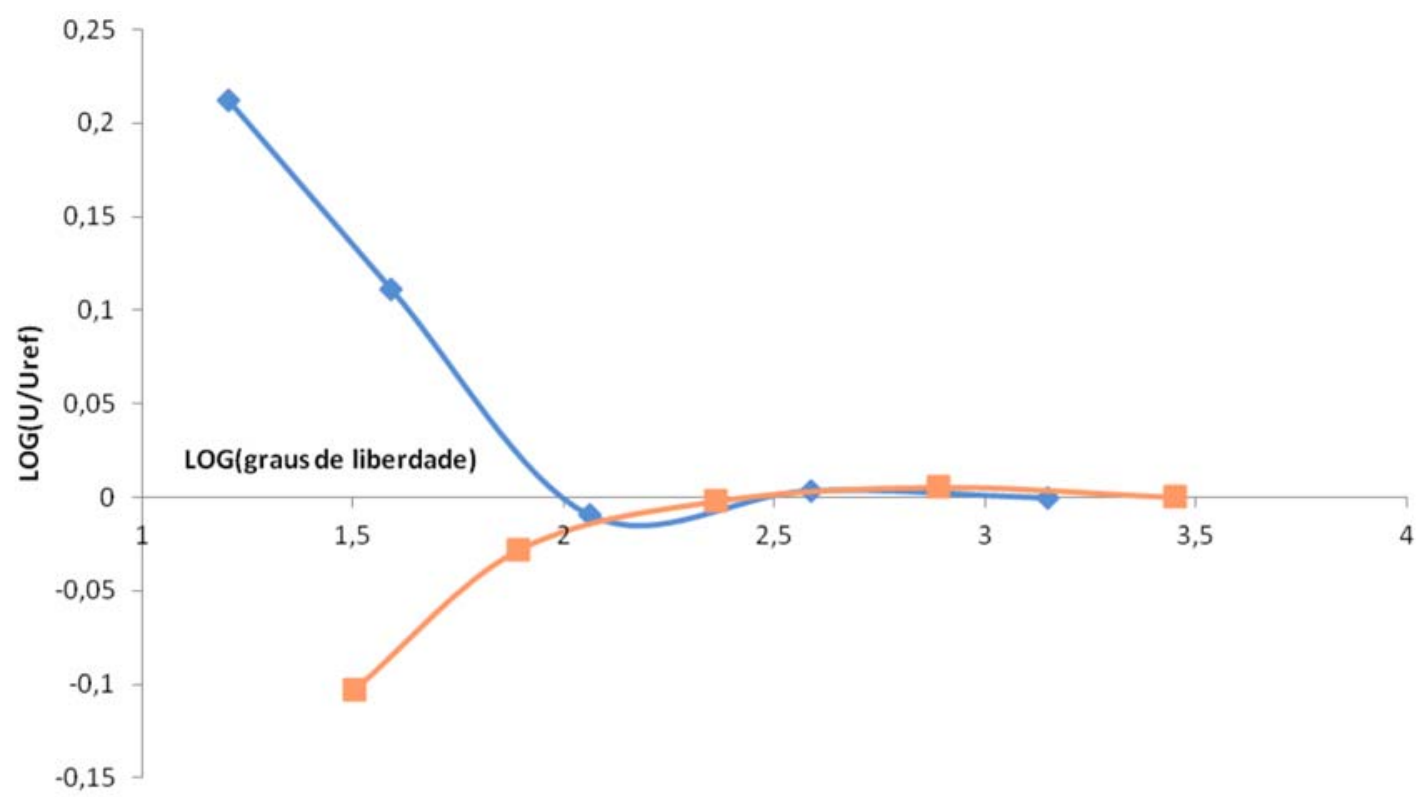

$\multimap$ FM-Sem enriquecimento $\quad-$ FM-Enriquecimento $(\mathrm{x})$ nas tensões e nos deslocamentos

Figura 6.6 - Verificação de convergência da FM(u- $\sigma)$.

Analisando a convergência da FHMT (Figura 6.5) observa-se que a aplicação de enriquecimento nos deslocamentos e tensões de domínio a simulação converge para um resultado apenas próximo da solução de referência. Já as simulações onde se enriqueceram apenas as tensões, ou as tensões e deslocamentos de contorno as soluções convergiram para a solução de referência. Analogamente, analisando a convergência da $\operatorname{FM}(u-\sigma)$ (Figura 6.6) 
observa-se que ambas as simulações apresentam boa convergência para a solução de referência.

Para fins de ilustração, na Figura 6.7 comparam-se os gráficos de tensão da FHMT (rede de 16x16 com enriquecimento em tensões de domínio e deslocamentos de contorno) com os da solução de referência.
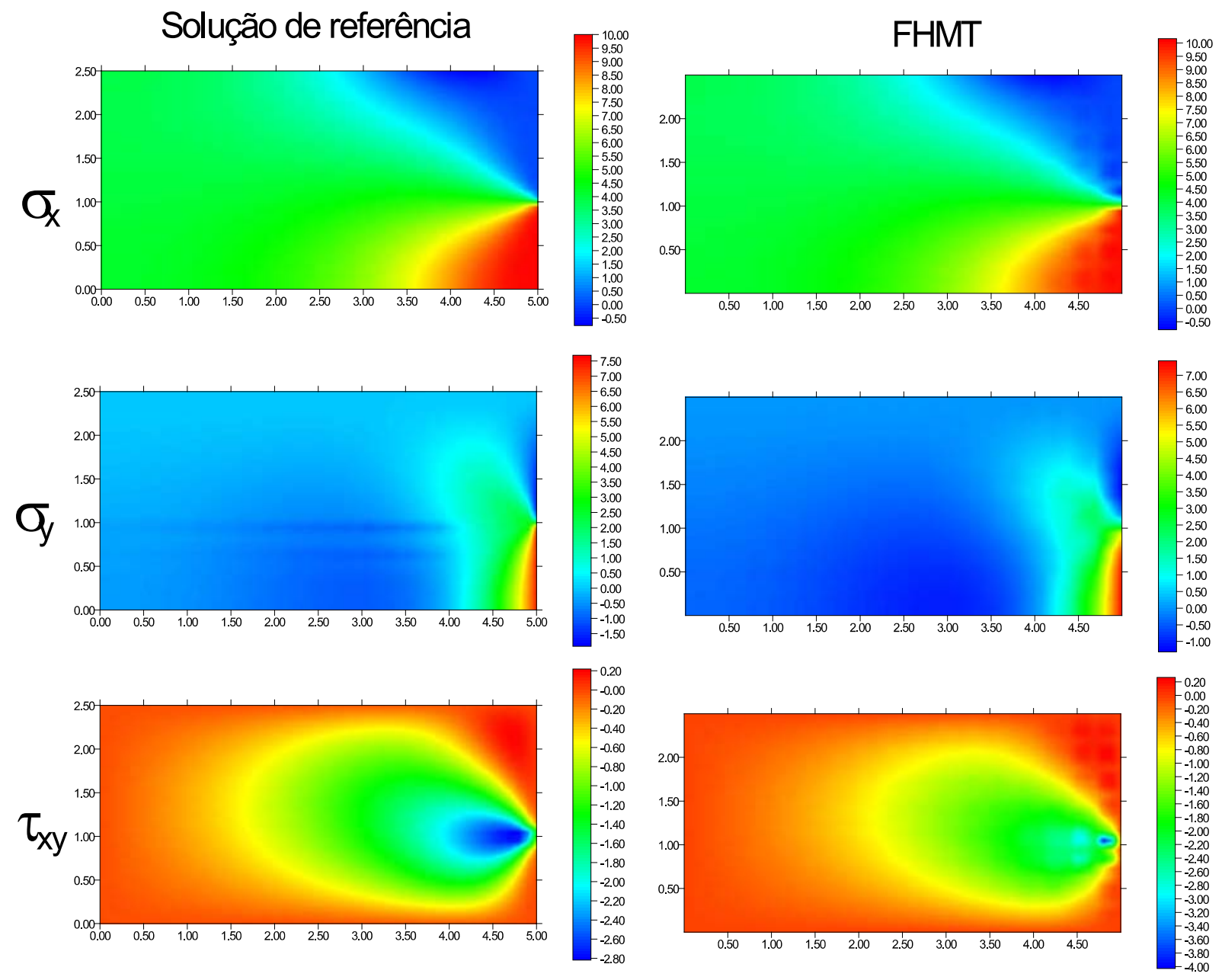

Figura 6.7 - Comparação entre a FHMT e a solução de referência.

Analogamente, a Figura 6.8 compara os gráficos de tensão da FM(u- $\sigma)$ (rede de 16x16 elementos com enriquecimento) com os da solução de referência. Nota-se que nesta simulação uma rede mais refinada seria necessária para prover melhores resultados na componente $\tau_{x y}$ de tensão. 
Resultados
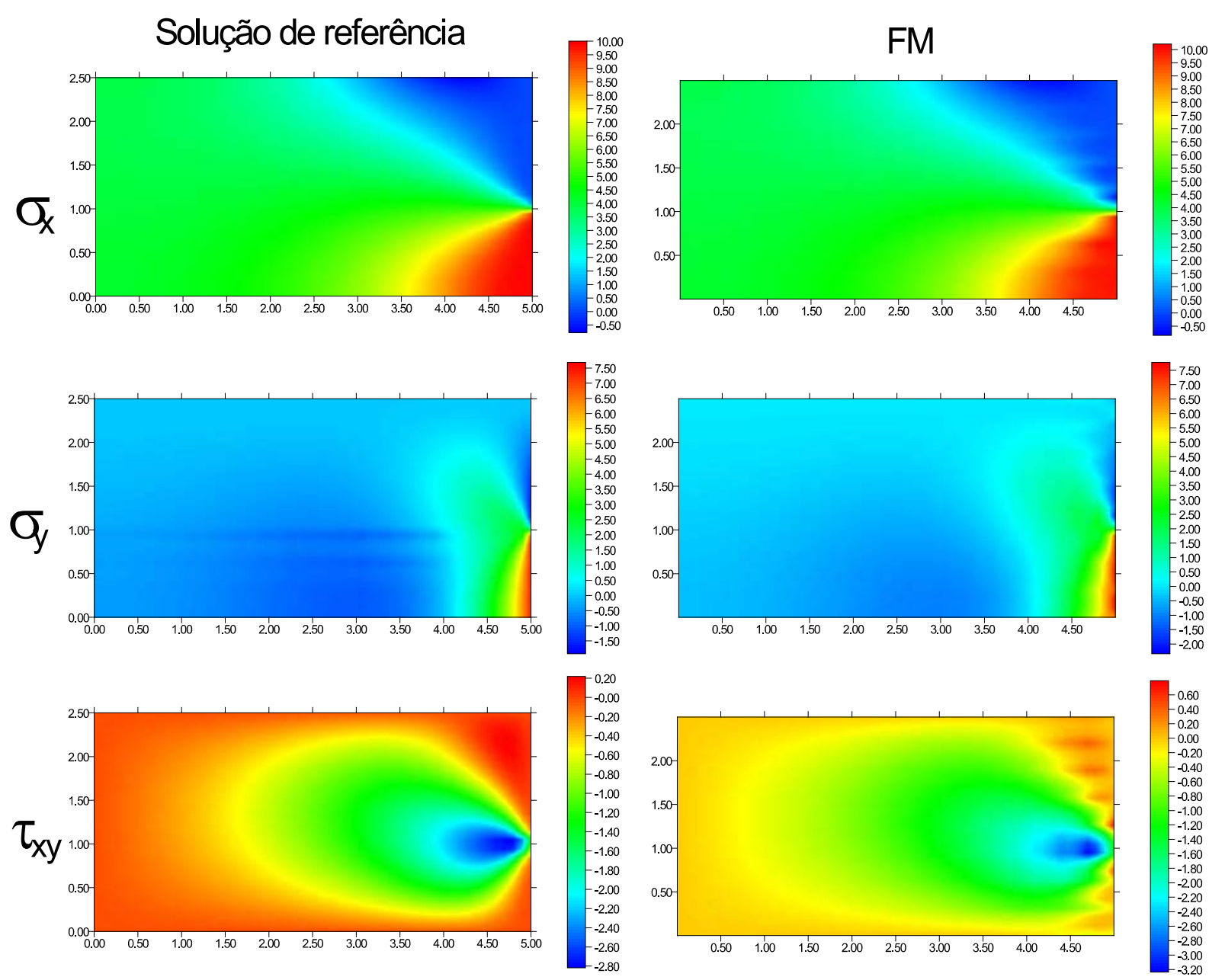

Figura 6.8 - Comparação entre a FM(u- $\sigma)$ e a solução de referência.

\subsection{Chapa tracionada com fissura discreta}

Este exemplo trata de uma chapa tracionada, com as mesmas dimensões do exemplo anterior, com uma fissura discreta em sua região central (Figura 6.9).

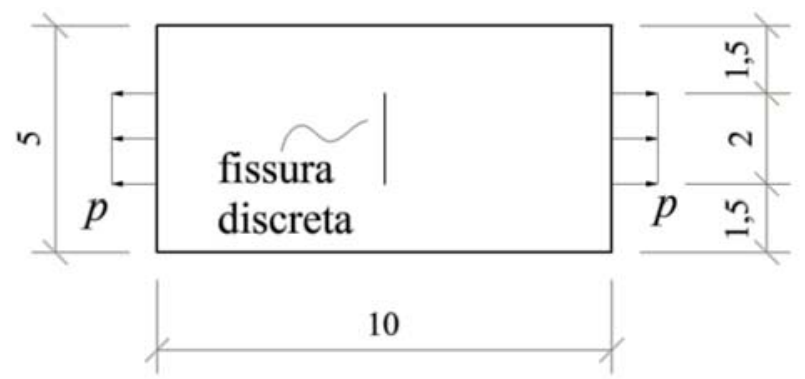

Figura 6.9 - Chapa tracionada com fissura discreta.

Dada a dupla simetria do problema, o modelo pode ser simplificado conforme a Figura 6.10. Ressalta-se que, assim como no exemplo anterior, esta chapa possui espessura unitária, 
$v=0,3, E=1000, \sigma_{0}=6, \sigma_{\infty}=10$ e está submetida a um carregamento uniforme parcialmente distribuído $p=10$.

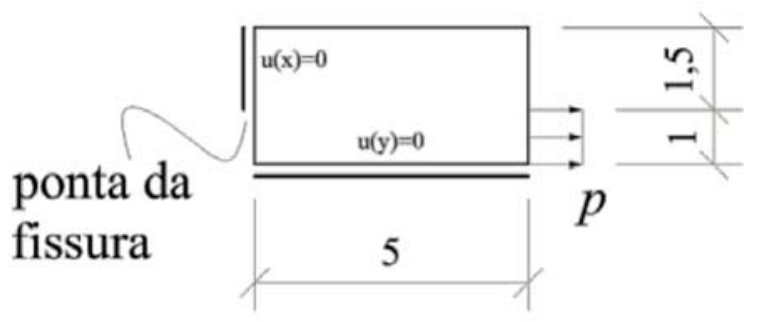

Figura 6.10 - Modelo matemático do problema.

Assim como no exemplo anterior, utilizou-se a ferramenta computacional ANSYS ${ }^{\circledR}$ (SAS, 2009) para a obtenção de uma solução de referência do problema, com uma rede irregular de, propositadamente densa, 15729 elementos finitos do tipo PLANE42 (quadrilateral de quatro nós). A rede apresenta maior concentração de elementos finitos na região da ponta da fissura.

Pela solução de referência observa-se que as tensões $\sigma_{x}$ (Figura 6.11), $\sigma_{y}$ (Figura 6.12) e $\tau_{x y}$ (Figura 6.13) possuem maiores valores absolutos próximo à ponta da fissura, como esperado.
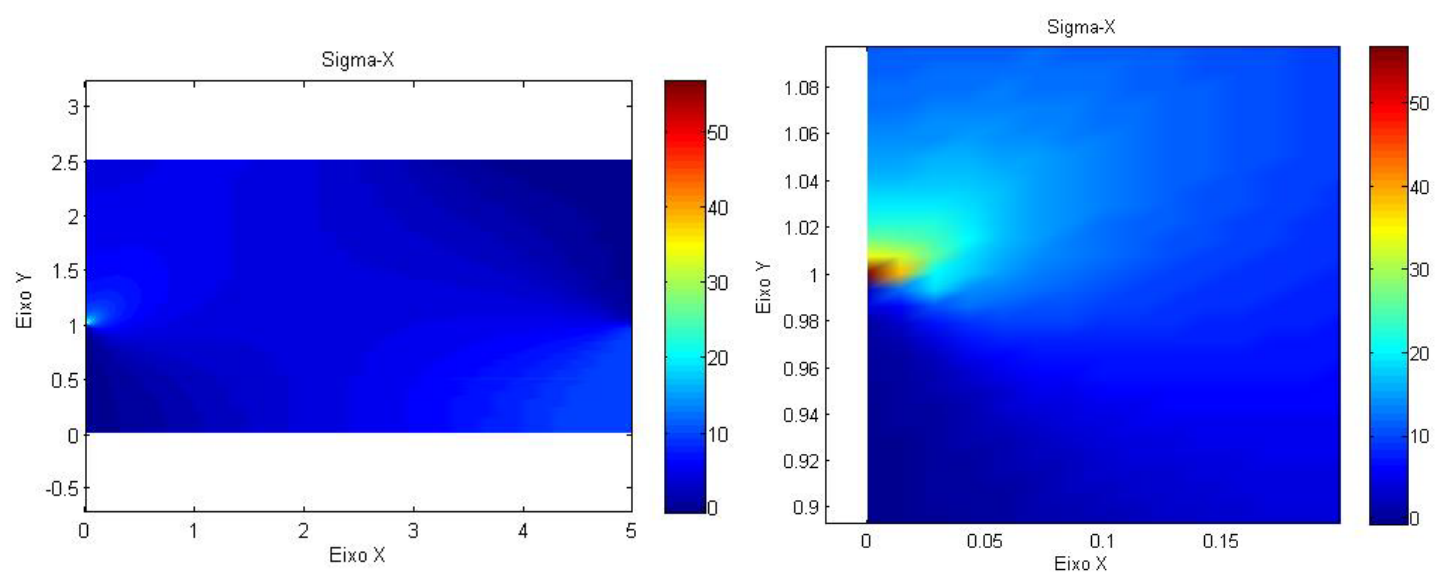

Figura 6.11 - Resultado de $\sigma_{x}$ da solução de referência para toda a chapa (esquerda) e detalhe da região da ponta da fissura (direita). 
Resultados
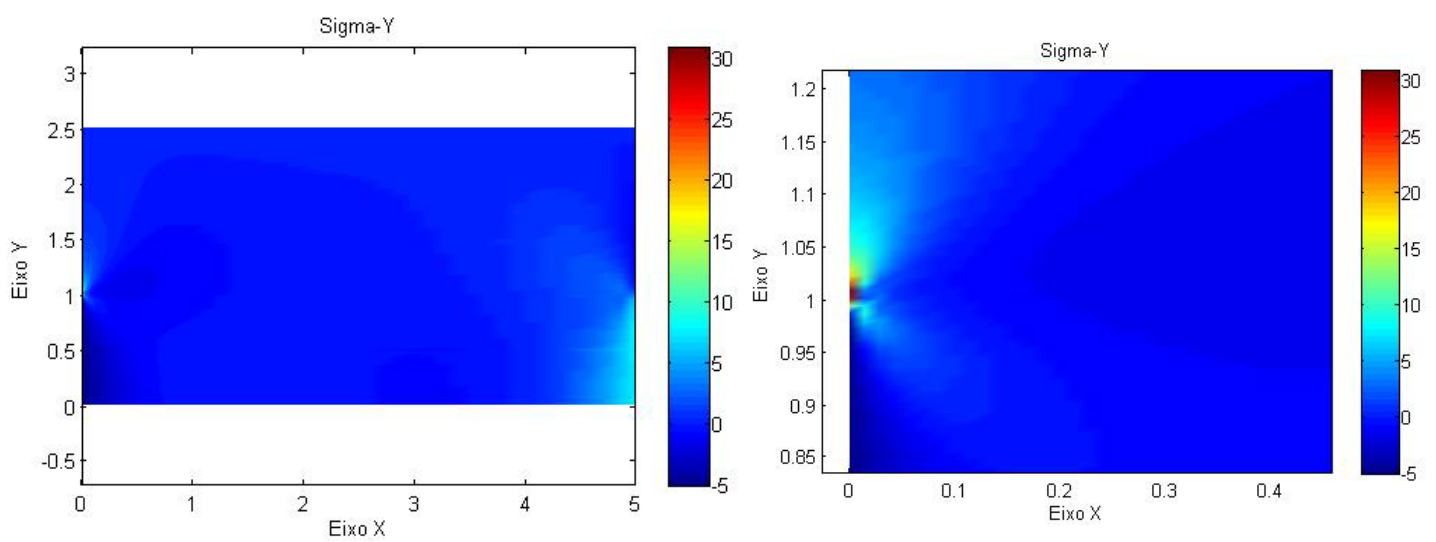

Figura 6.12 - Resultado de $\sigma_{y}$ da solução de referência para toda a chapa (esquerda) e detalhe da região da ponta da fissura (direita).
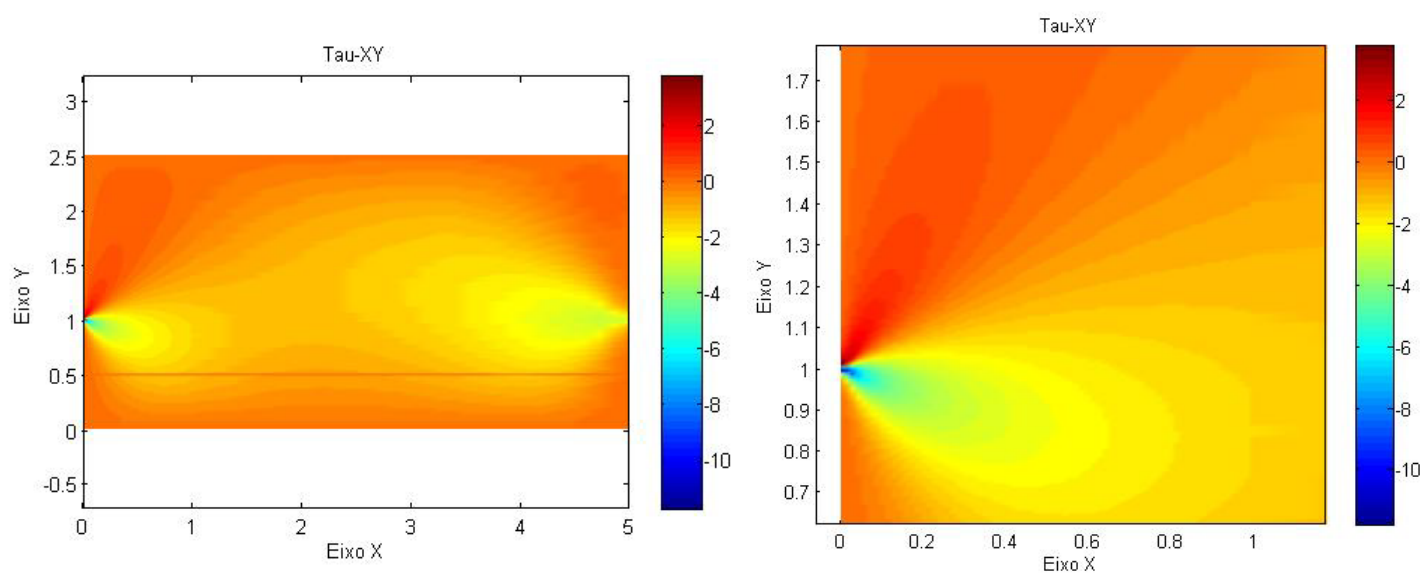

Figura 6.13 - Resultado de $\tau_{x y}$ da solução de referência para toda a chapa (esquerda) e detalhe da região da ponta da fissura (direita).

De posse dos resultados de tensão da solução de referência, calculou-se, também, a distribuição de dano para o problema (Figura 6.14) utilizando-se do modelo desacoplado simplificado (item 5.4). 


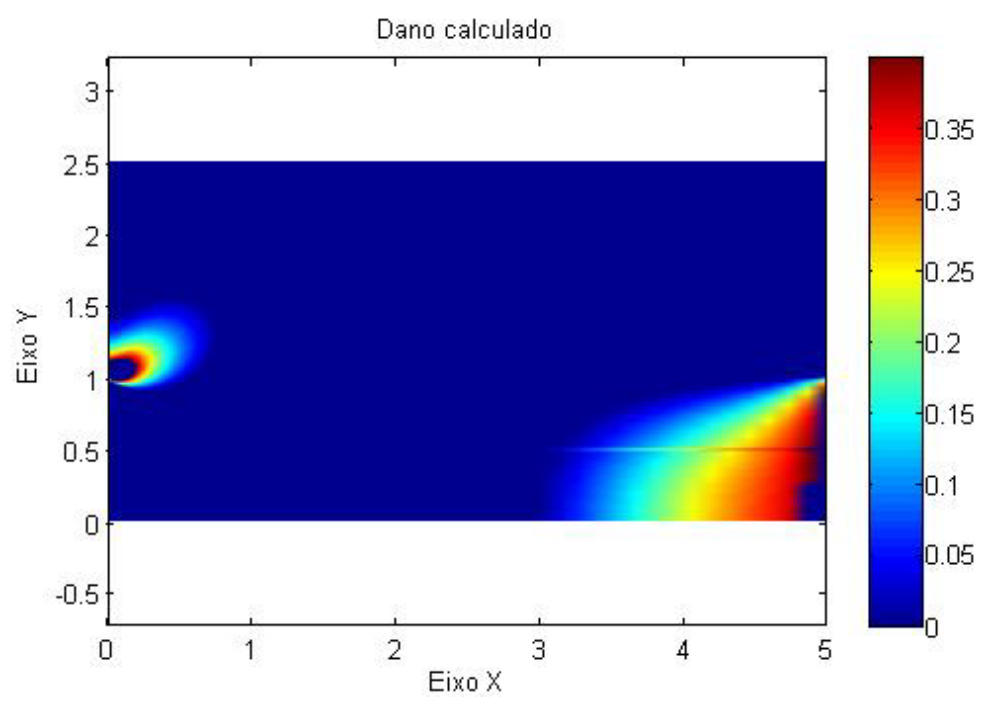

Figura 6.14 - Dano calculado utilizando-se a solução de referência.

Ressalta-se, tanto para a solução de referência quanto para as aproximações em estudo, que na região de aplicação da carga distribuída o valor de dano é nulo, pois estes pontos ultrapassaram o limite de ruptura considerado. O mesmo vale para os pontos próximos à ponta da fissura.

A fim de reproduzir os resultados de referência para este exemplo, que possui uma singularidade, foram realizadas três simulações, uma com o MEFG, outra com a FHMT e outra com a $\operatorname{FM}(\mathrm{u}-\sigma)$.

Assim, a análise com o MEFG foi conduzida utilizando-se uma discretização de 32x32 elementos quadrilaterais de quatro nós, conforme indicado na Figura 6.15.

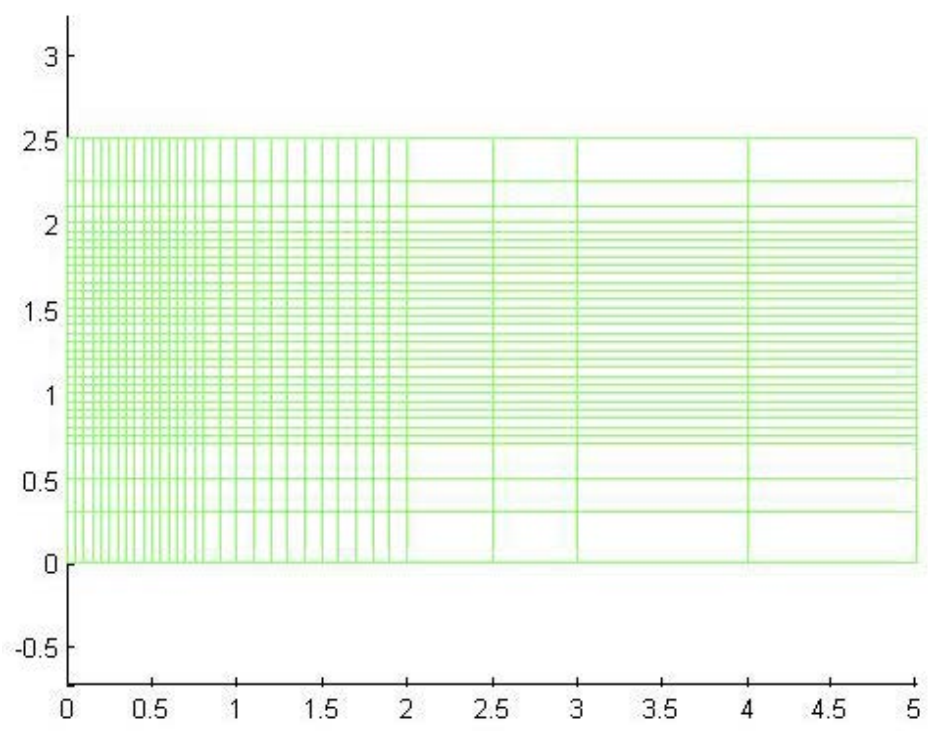

Figura 6.15 - Discretização adotada para simulação com o MEFG. 
Os resultados para tensões $\sigma_{x}$ (Figura 6.16), $\sigma_{y}$ (Figura 6.17) e $\tau_{x y}$ (Figura 6.18), bem como o dano calculado (Figura 6.19), foram obtidos utilizando-se um polinômio completo do primeiro grau $\left(L_{3(\alpha)}=\{1, x, y\}\right)$ como função de enriquecimento, totalizando 6477 graus de liberdade.
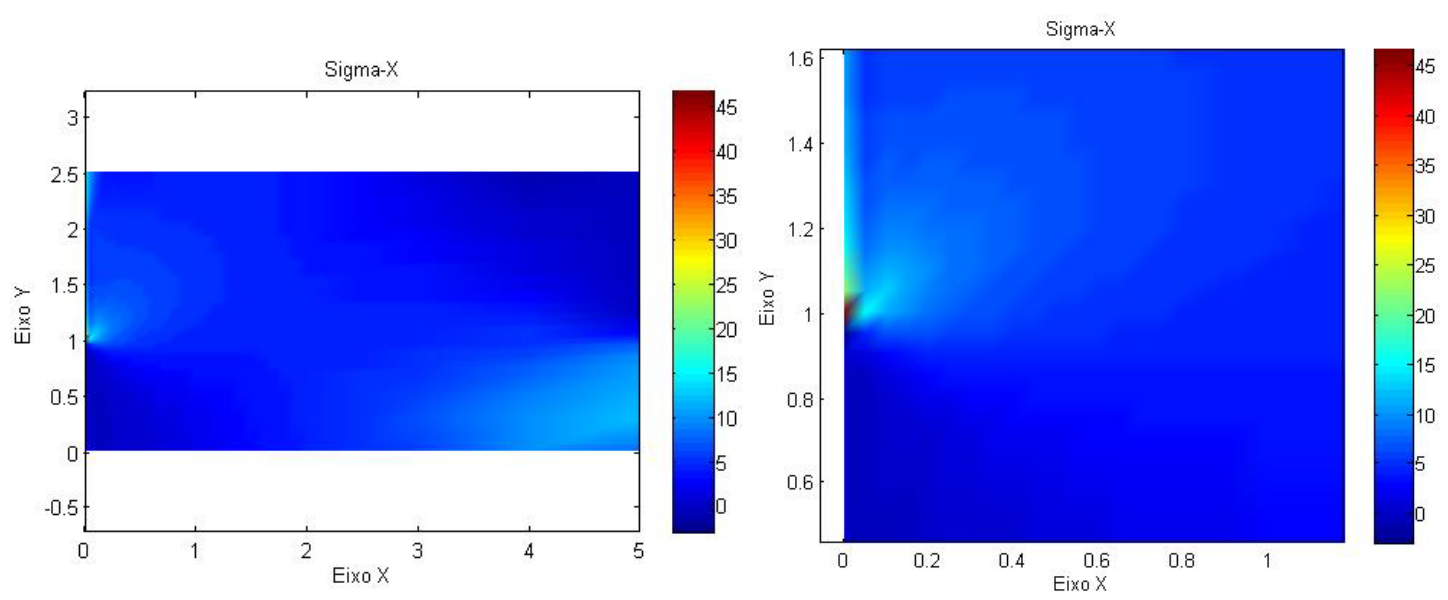

Figura 6.16 - Resultado de $\sigma_{x}$ do MEFG para toda a chapa (esquerda) e detalhe da região da ponta da fissura (direita).
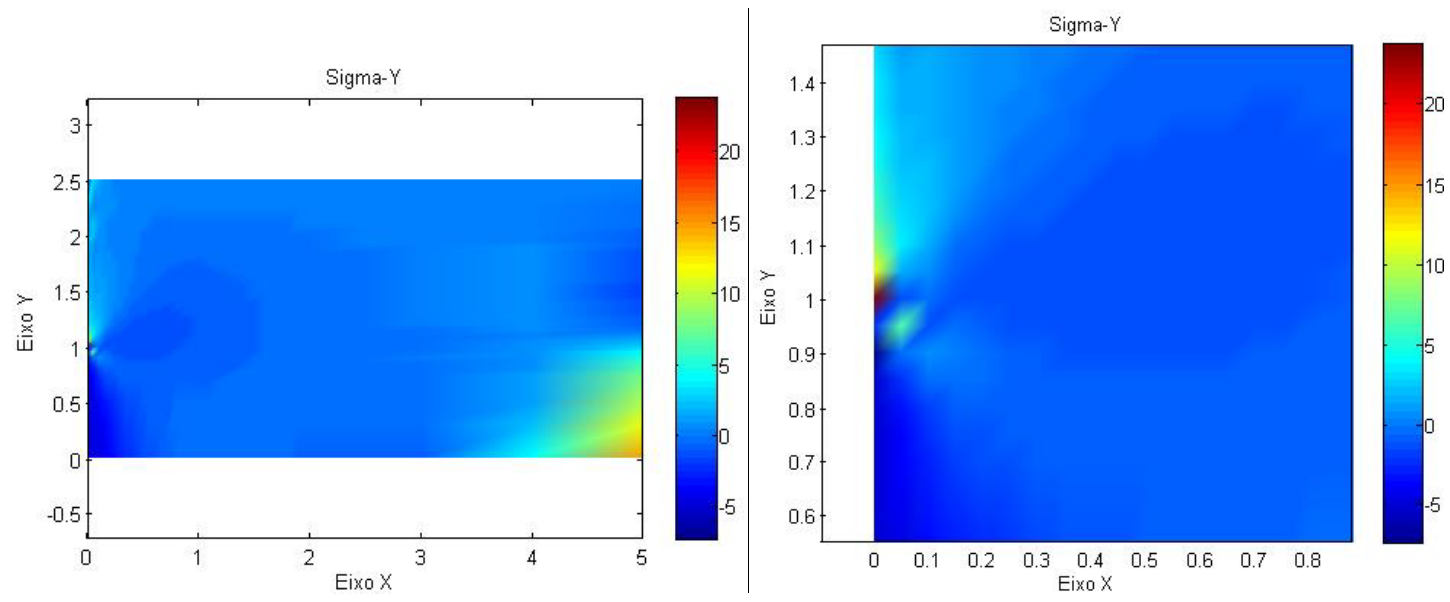

Figura 6.17 - Resultado de $\sigma_{y}$ do MEFG para toda a chapa (esquerda) e detalhe da região da ponta da fissura (direita). 

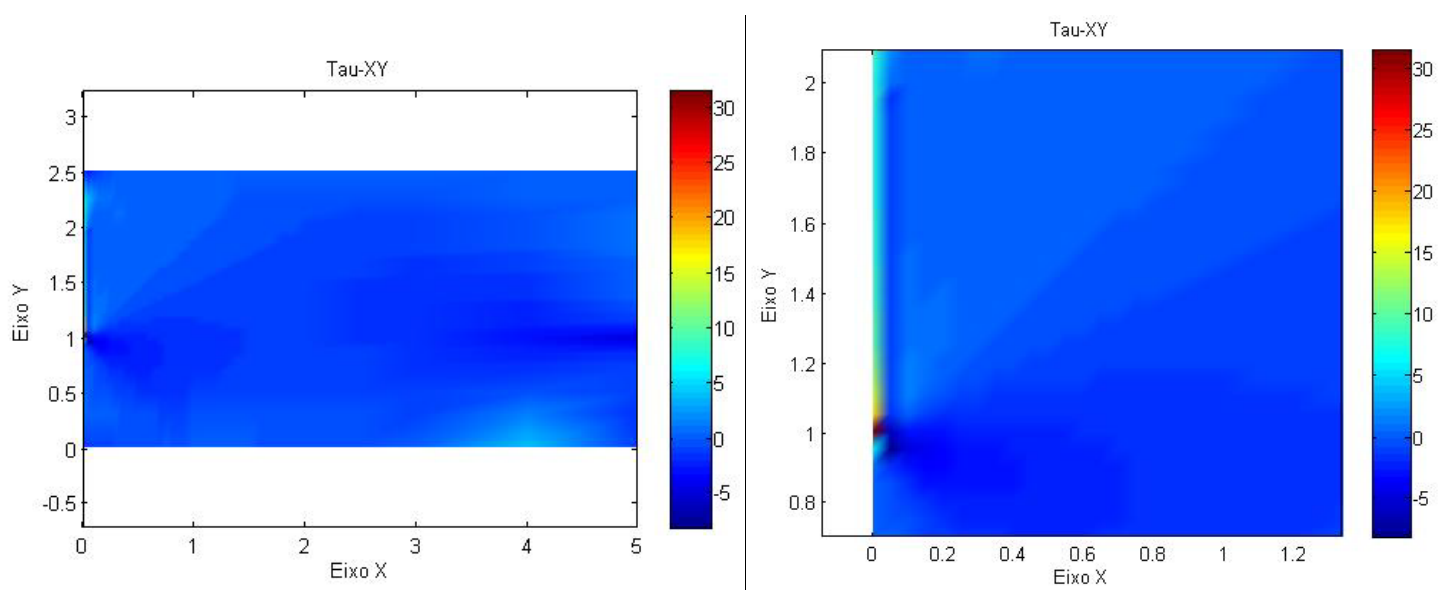

Figura 6.18 - Resultado de $\tau_{x y}$ do MEFG para toda a chapa (esquerda) e detalhe da região da ponta da fissura (direita).

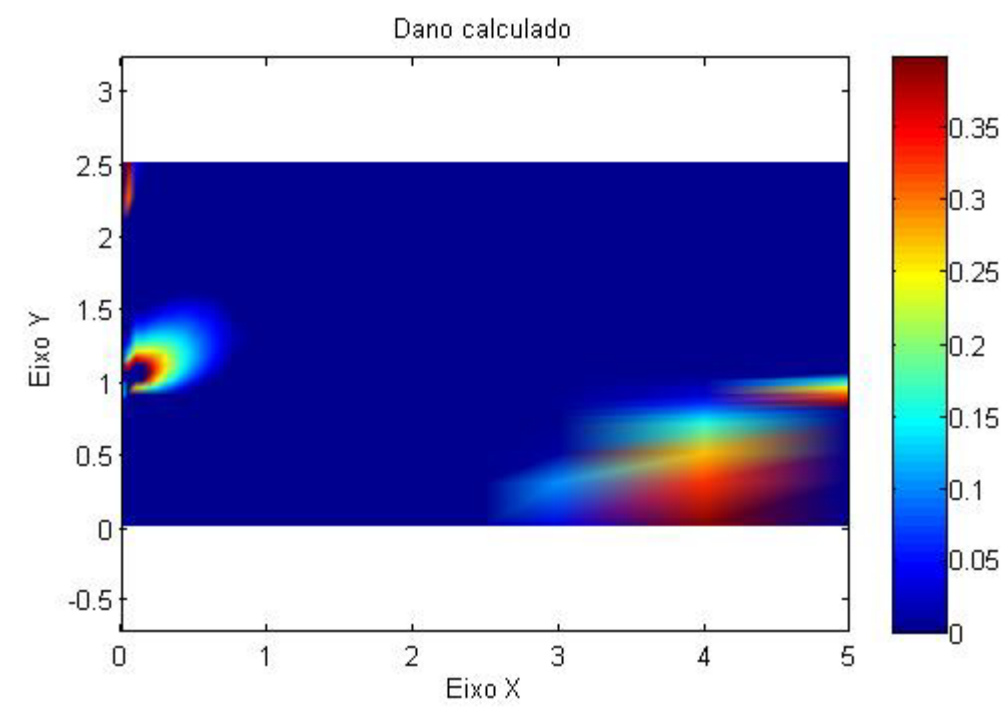

Figura 6.19 - Dano calculado para a solução do MEFG.

As outras duas simulações foram realizadas com as formulações propostas, utilizando uma discretização de $24 \times 24$ elementos para a FHMT e de $32 \times 32$ para a FM(u- $\sigma)$ (idêntica à escolhida para o MEFG), conforme mostrado na Figura 6.20. 
Resultados
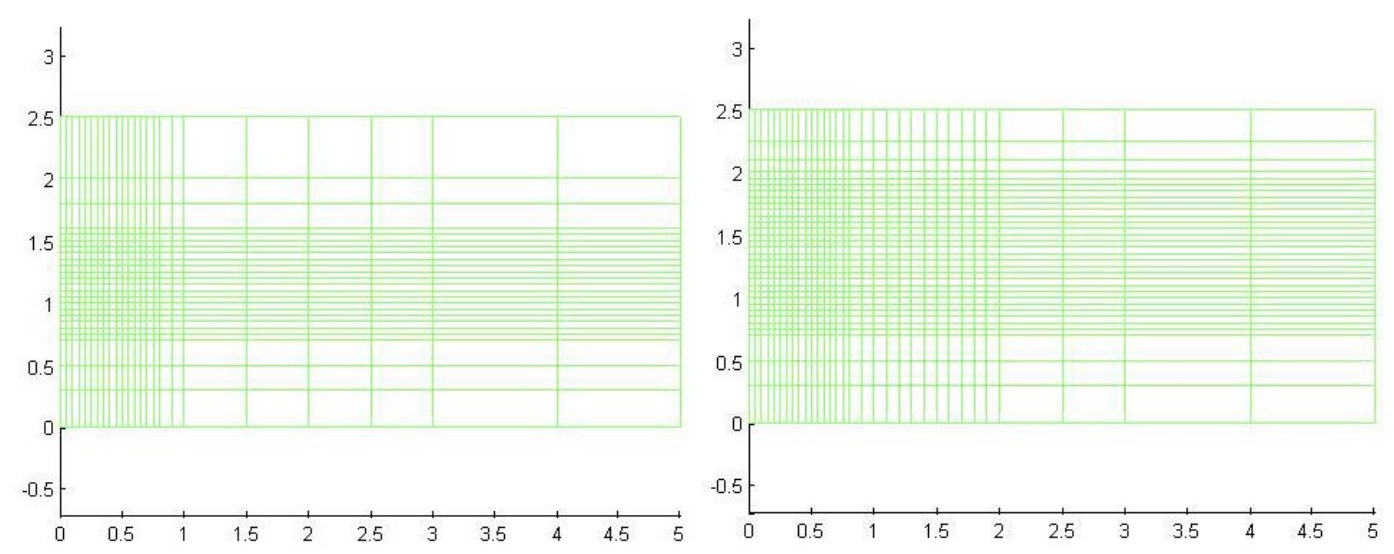

Figura 6.20 - Discretizações adotadas para simulação com a FHMT (esquerda) e FM(u- $\sigma$ ) (direita).

Os resultados das tensões $\sigma_{x}$ (Figura 6.21), $\sigma_{y}$ (Figura 6.22) e $\tau_{x y}$ (Figura 6.23) para a simulação realizada com a FHMT foram obtidos com a adoção de $L_{2(\alpha)}^{\Omega}=\{1, x y\}$ como função de enriquecimento apenas para os campos de tensão no domínio, totalizando 6170 graus de liberdade. O dano calculado com os resultados da FHMT pode ser observado na Figura 6.24 .
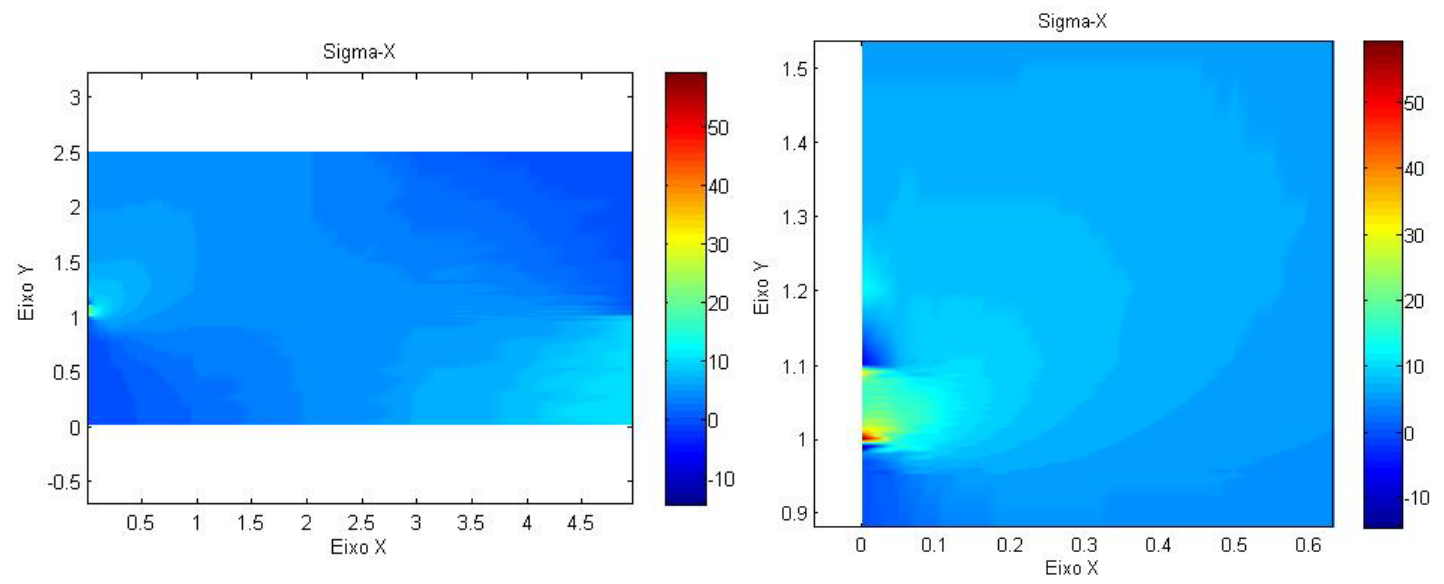

Figura 6.21 - Resultado de $\sigma_{x}$ da FHMT para toda a chapa (esquerda) e detalhe da região da ponta da fissura (direita). 

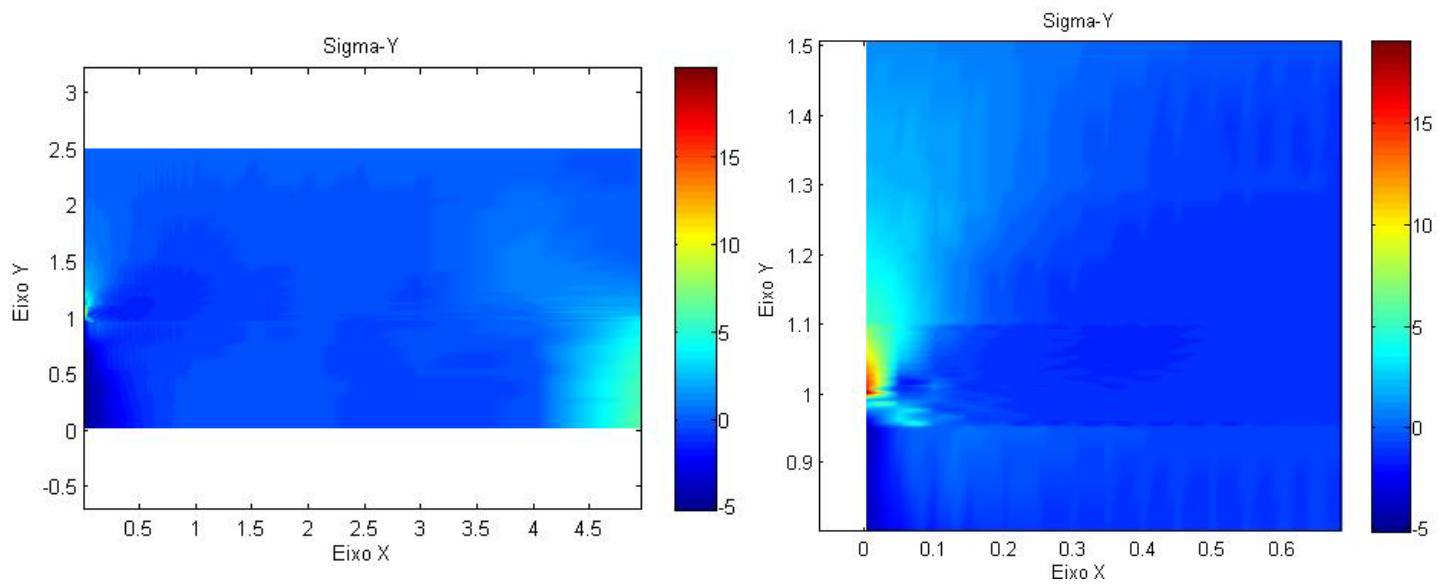

Figura 6.22 - Resultado de $\sigma_{y}$ da FHMT para toda a chapa (esquerda) e detalhe da região da ponta da fissura (direita).
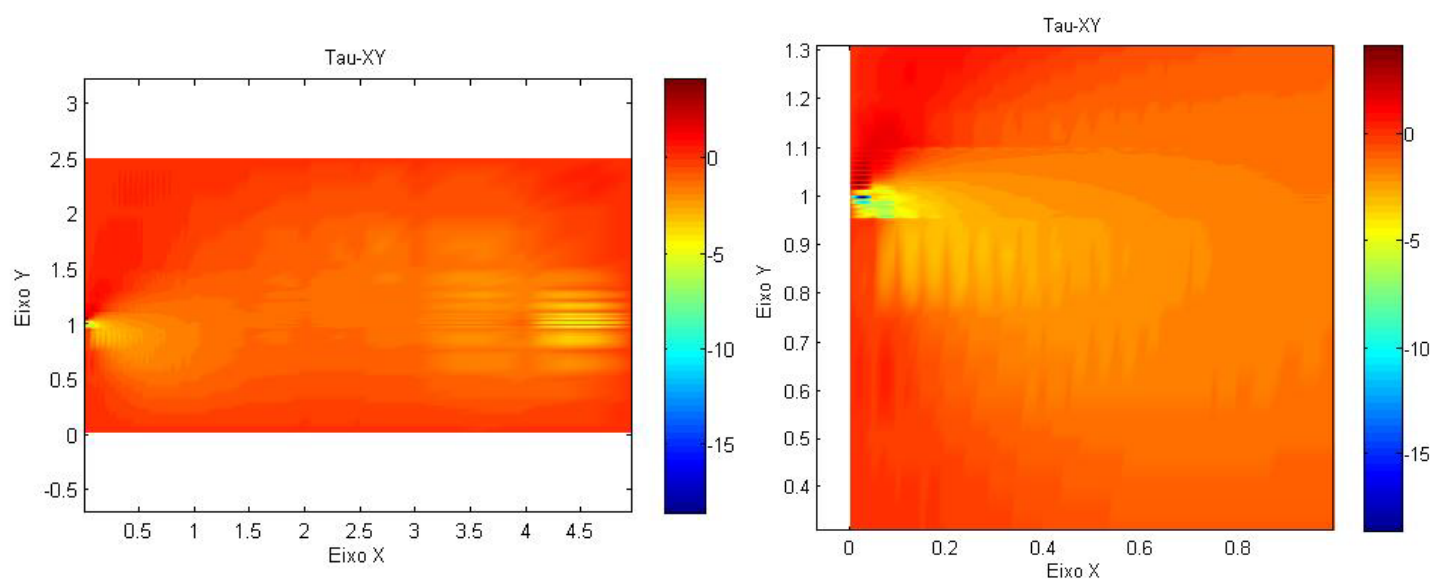

Figura 6.23 - Resultado de $\tau_{x y}$ da FHMT para toda a chapa (esquerda) e detalhe da região da ponta da fissura (direita).

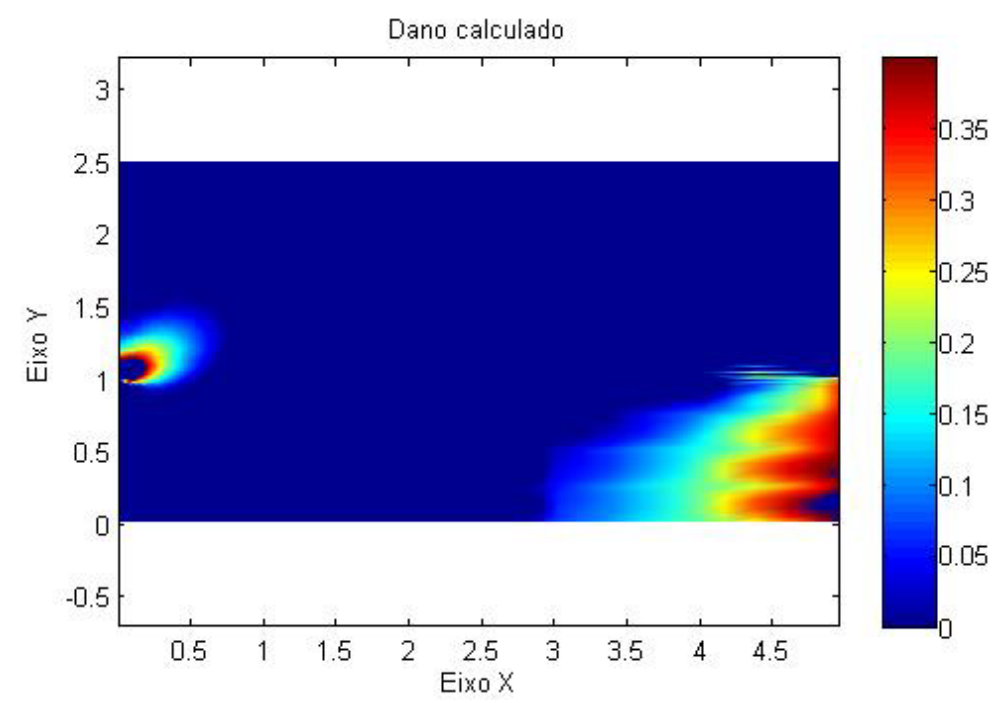

Figura 6.24 - Dano calculado utilizando-se a FHMT. 
Resultados

Os resultados das tensões $\sigma_{x}$ (Figura 6.25), $\sigma_{y}$ (Figura 6.26) e $\tau_{x y}$ (Figura 6.27) para a simulação realizada com a $\operatorname{FM}(\mathrm{u}-\sigma)$ foram obtidos sem a adoção de funções de enriquecimento, pois nenhuma das funções testadas apresentou aproximação satisfatória à solução de referência, totalizando 5388 graus de liberdade. O dano calculado da FM(u- $\sigma)$ pode ser observado na Figura 6.28Figura 6.24.
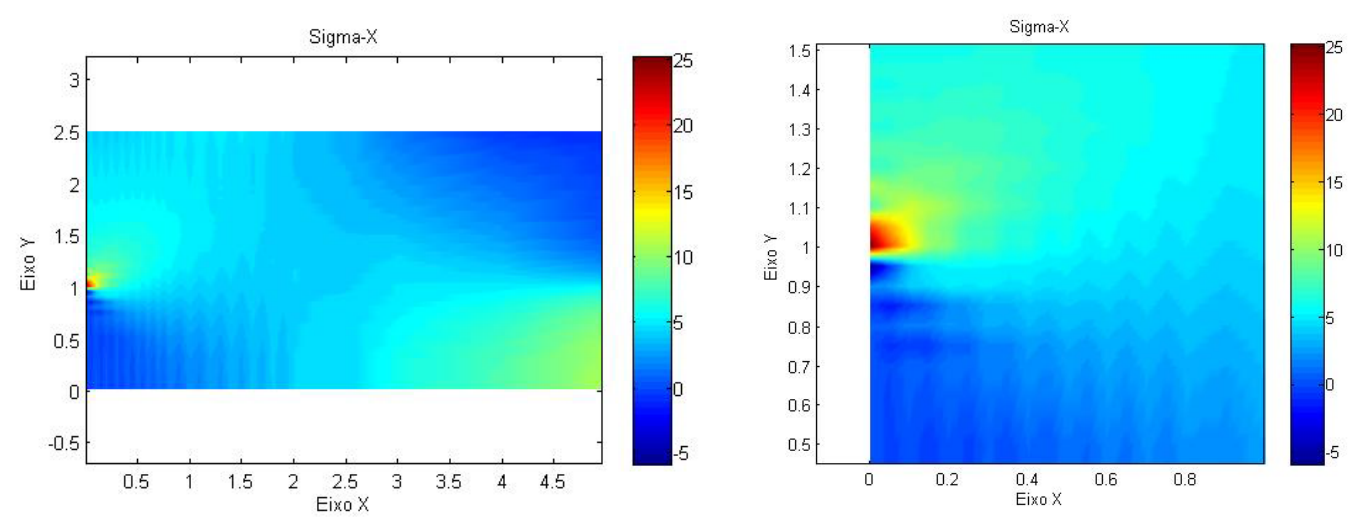

Figura 6.25 - Resultado de $\sigma_{x}$ da FM(u- $\left.\sigma\right)$ para toda a chapa (esquerda) e detalhe da região da ponta da fissura (direita).
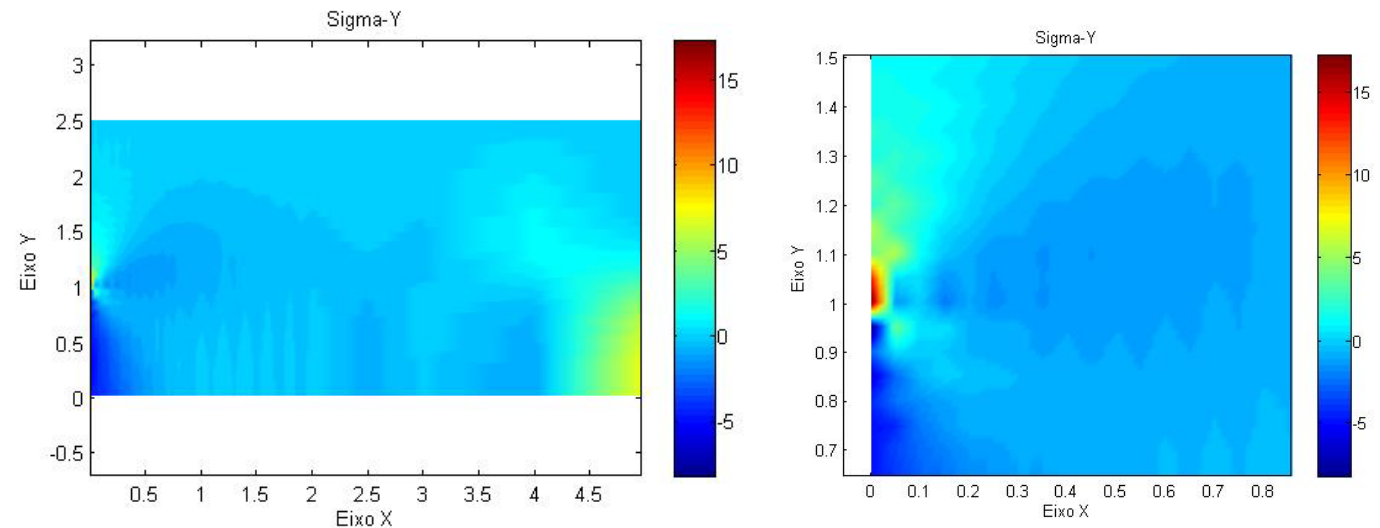

Figura 6.26 - Resultado de $\sigma_{y}$ da $\mathrm{FM}(\mathrm{u}-\sigma)$ para toda a chapa (esquerda) e detalhe da região da ponta da fissura (direita). 

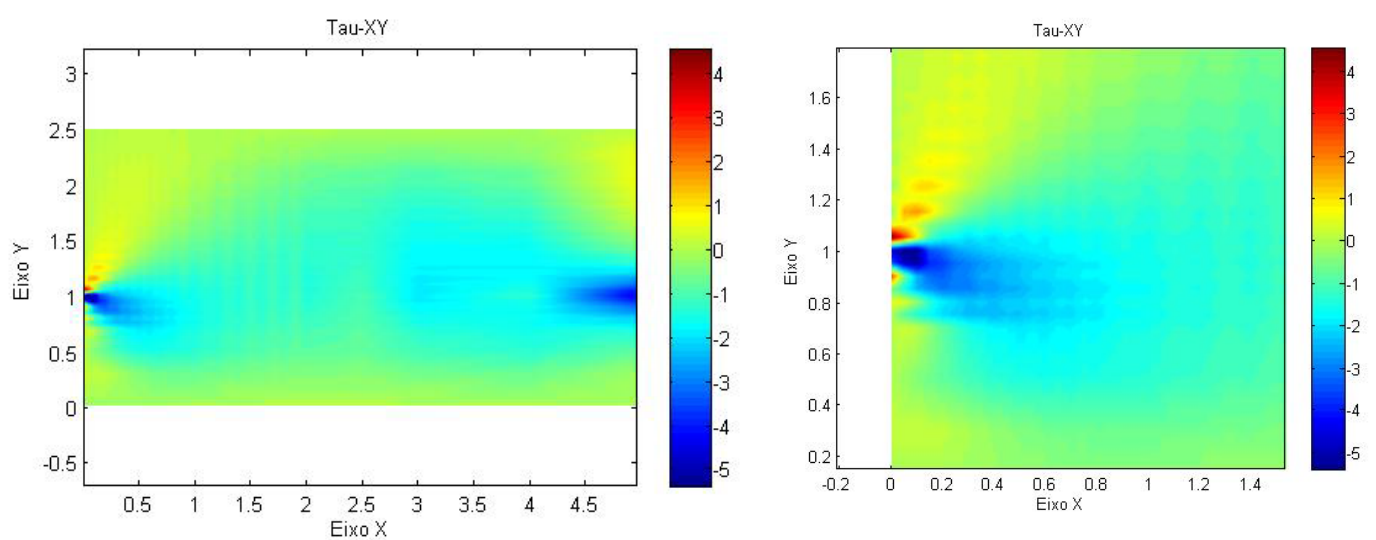

Figura 6.27 - Resultado de $\tau_{x y}$ da FM(u- $\left.\sigma\right)$ para toda a chapa (esquerda) e detalhe da região da ponta da fissura (direita).

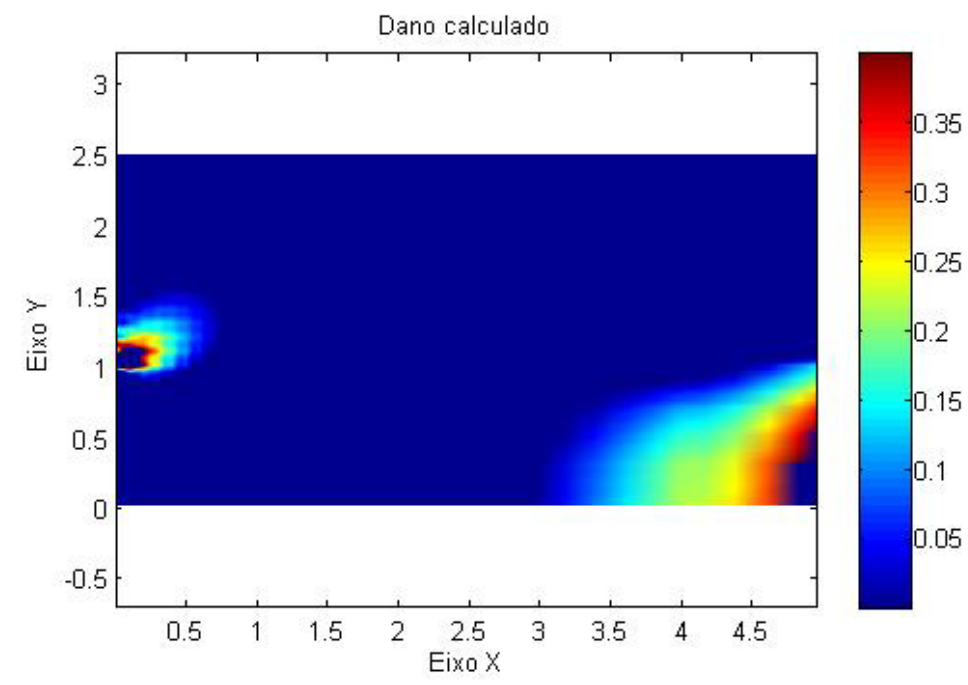

Figura 6.28 - Dano calculado utilizando-se a FM(u- $\sigma)$.

Diante do exposto observa-se que, para este exemplo, as formulações propostas obtiveram resultados bastante bons em comparação com a solução de referência admitida.

Acredita-se que as formulações FHMT e $\mathrm{FM}(\mathrm{u}-\sigma)$ propostas obtiveram bom desempenho em razão da sua melhor capacidade de aproximação dos campos de tensão.

Ressalta-se, novamente, que a distribuição de dano apresentada neste exemplo foi obtida pela análise desacoplada simplificada, descrita no item 5.4. De fato, esta distribuição neste ponto serve apenas para uma prévia comparação entre as formulações. Assim, nos exemplos seguintes realiza-se uma análise não linear com dano mais completa para verificar até que ponto o modelo simplificado é representativo. 
Resultados

\subsection{Teste de tração}

Este exemplo trata de um teste de tração de uma peça de concreto simples, com dimensões $\mathrm{H}=20 \mathrm{~mm}, \mathrm{~L}=100 \mathrm{~mm}$ e espessura unitária, e carregamento $p=3,3 \mathrm{MPa}$ aplicado de forma monotônica numa das extremidades, conforme apresentado na Figura 6.29. Vale salientar que neste exemplo não se faz uso da técnica de enriquecimento nodal, pois se pretende avaliar o comportamento de cada formulação em resposta a uma análise não linear acoplada com dano.

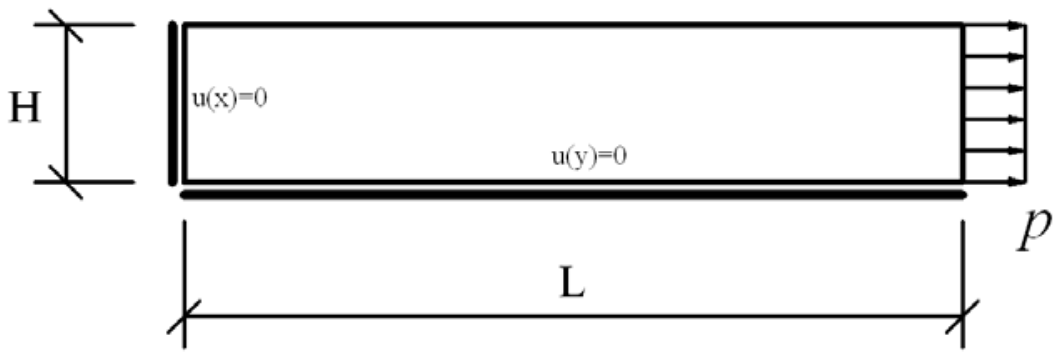

Figura 6.29 - Teste de tração numa chapa.

Admite-se que para o concreto adotado, foram identificados de acordo com o modelo de dano de Comi e Perego (2001) os seguintes parâmetros físicos: $E=30 \mathrm{GPa}, n=3$, $k=0,00015 \mathrm{MPa}$ e $c=2,72$.

Primeiramente adotou-se $v=0$ para realizar um estudo comparativo das formulações com a solução analítica para o ensaio de tração uniaxial (Figura 6.30).

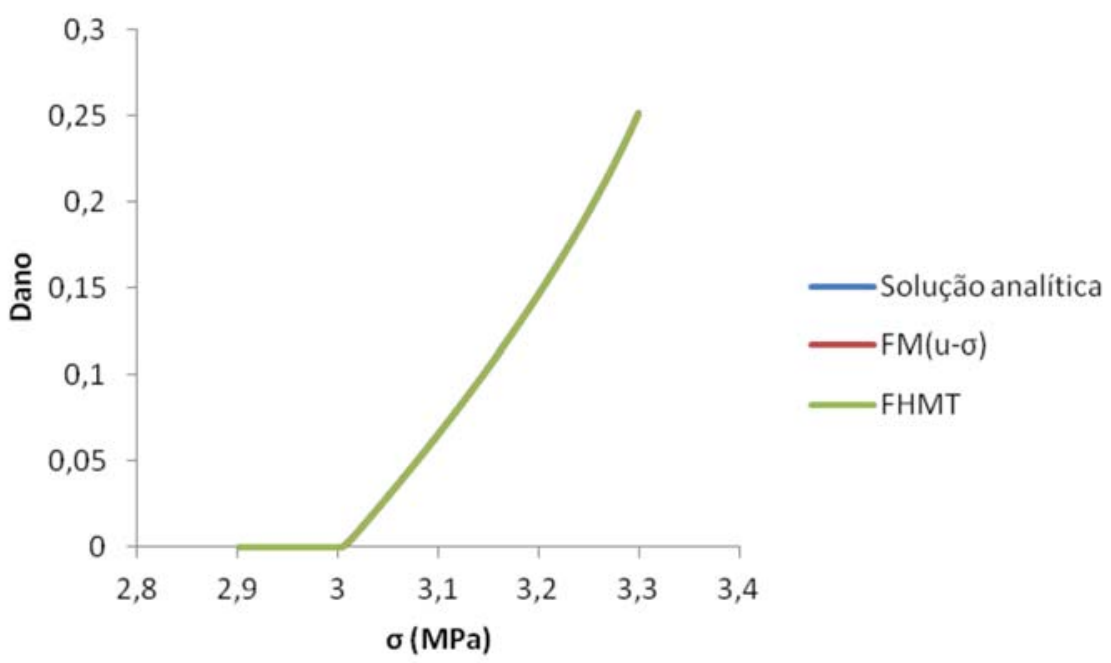

Figura 6.30 - Comparação das formulações com a solução analítica. 
Observa-se que as formulações apresentadas acompanharam a solução analítica do problema. Claramente ambas as formulações reproduzem de forma satisfatória o comportamento uniaxial do ensaio proposto. Entretanto, tendo-se em vista as análises planas, optou-se por uma verificação da influência do coeficiente de Poisson no modelo.

Inicialmente, foram realizadas análises não lineares para a situação de estado plano de tensões (EPT). Como esperado, concluiu-se que ambas as formulações, é reproduzida a resposta da Figura 6.30, independente do valor do coeficiente de Poisson. Nas análises não lineares em estado plano de deformações (EPD), notou-se a influência da variação do valor do coeficiente de Poisson tanto para a FHMT (Figura 6.31) quanto para a FM(u- $\sigma$ ) (Figura 6.32).

\section{FHMT}

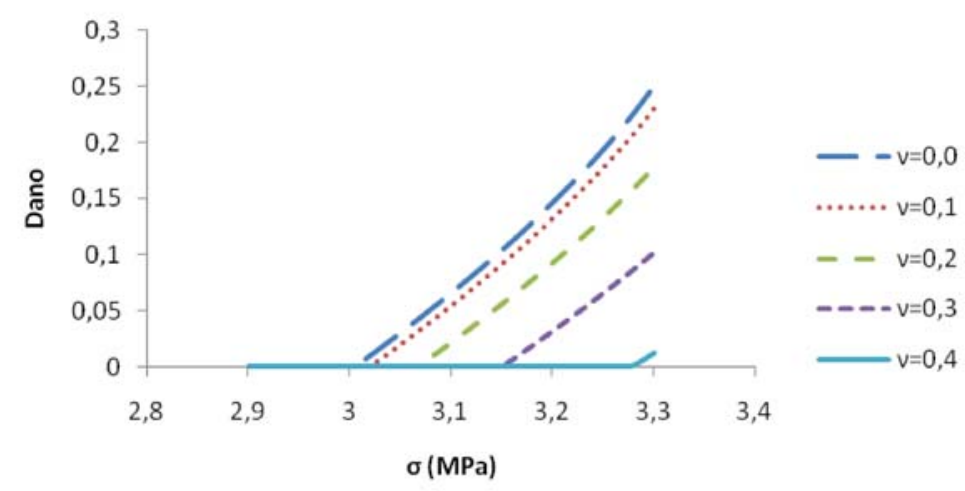

Figura 6.31 - Influência do coeficiente de Poisson na resposta não linear da FHMT em EPD.

\section{$\operatorname{FM}(u-\sigma)$}

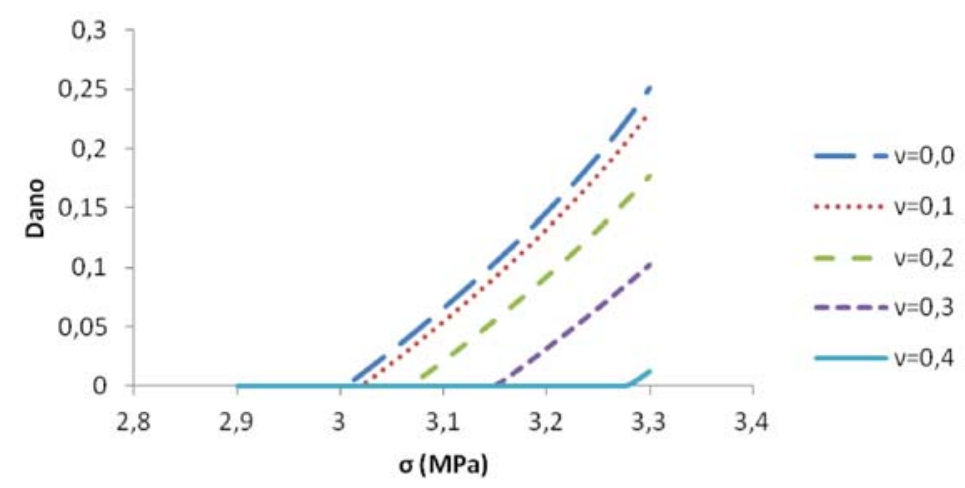

Figura 6.32 - Influência do coeficiente de Poisson na resposta não linear da FM(u- $\sigma)$ em EPD.

Observa-se que, em ambas as formulações, o coeficiente de Poisson acaba por governar o limiar de início de dano. 
Resultados

O exemplo em questão é agora utilizado para verificar até que ponto a forma simplificada de dano apresenta respostas aceitáveis no confronto com a análise não linear completa. Retoma-se o EPT $\operatorname{com} v=0$. Ressalta-se que se adotou $D_{c}=0,4$ como critério crítico de dano quando da utilização do modelo simplificado.

Para melhor visualização das respostas de dano, define-se $\delta D$ como um escalar calculado pela seguinte relação:

$\delta D=\log \left(\frac{D^{S I M P L}}{D^{N L I N}}\right)$

onde $D^{S I M P L}$ e $D^{N L I N}$ são as respostas de dano obtidas pelas análises simplificada e não linear, respectivamente. Com isso, os resultados são apresentados na Figura 6.33, para a FHMT, e na Figura 6.34, para a FM(u- $\sigma)$.

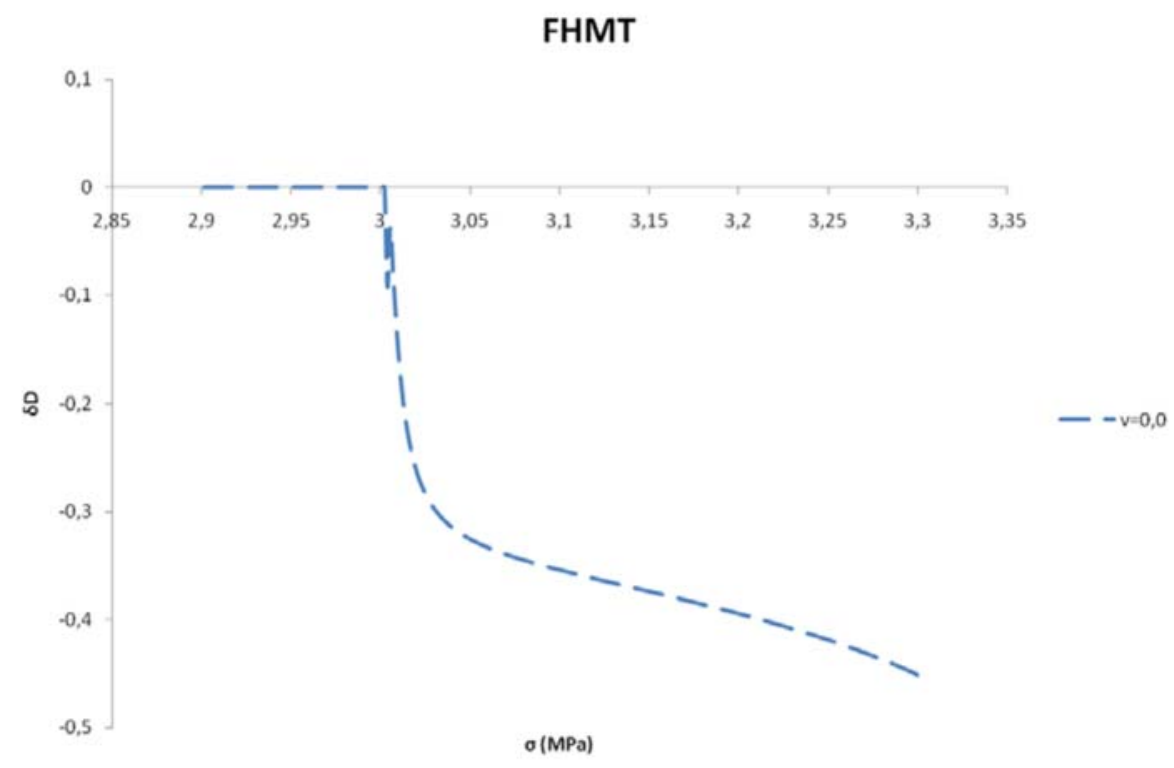

Figura 6.33 - Confronto das respostas de dano para a FHMT. 


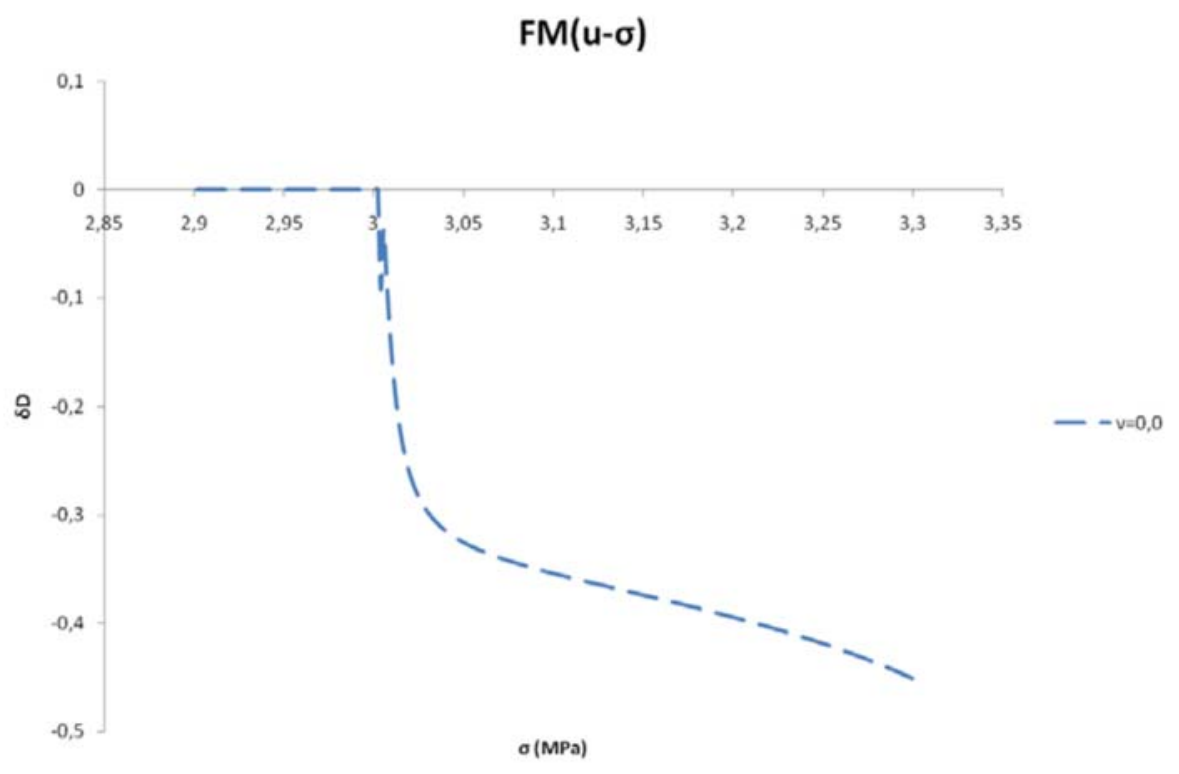

Figura 6.34 - Confronto das respostas de dano para a FM(u- $\sigma)$.

Observa-se que em ambas as formulações os valores de dano obtidos pelo modelo simplificado são satisfatórios frente aos valores obtidos com o modelo totalmente acoplado (não linear) somente numa estreita faixa de tensão logo após o aparecimento do dano. Entretanto, esta condição era esperada, dado que a danificação ocorreu em todo o elemento estrutural. Nos exemplos a seguir avalia-se o modelo simplificado em situações de geometria e vinculação que propiciem danificação em regiões mais localizadas do elemento estrutural.

\subsection{Teste de tração não uniforme}

Nos exemplos anteriores observou-se que as formulações propostas apresentam resultados muito próximos ou idênticos. Assim, a partir daqui utiliza-se apenas a FHMT, pois o objetivo nestes últimos exemplos é simplesmente de comparar as abordagens simplificada ou não de dano, conforme previamente mencionado.

Assim como no exemplo anterior, este teste consiste de uma peça de concreto simples em tração, com dimensões $\mathrm{H}=20 \mathrm{~mm}, \mathrm{~L}=100 \mathrm{~mm}$ e espessura unitária. $\mathrm{O}$ carregamento $p=6,95 \mathrm{MPa}$ é aplicado de forma monotônica numa faixa $\mathrm{a}=3 \mathrm{~mm}$ de uma das extremidades, conforme apresentado na Figura 6.35. Utiliza-se um concreto com $E=30 \mathrm{GPa}$, $n=3, k=0,00015 \mathrm{MPa}, c=2,72$ e $v=0$. 
Resultados

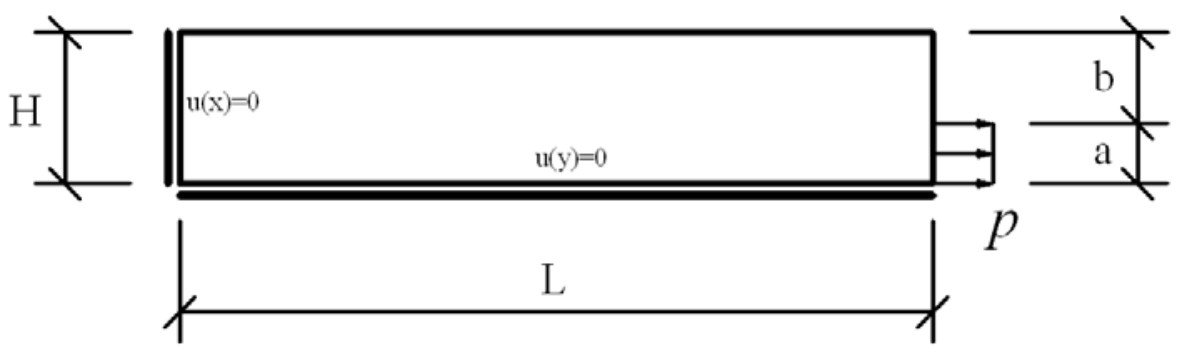

Figura 6.35 - Teste de tração localizada.

A discretização foi feita com oito elementos finitos híbrido-mistos dispostos horizontalmente e utilizaram-se as funções de enriquecimento $L_{2(\alpha)}^{\Omega}=\left\{1, y^{2}\right\}$ e $L_{2(\alpha)}^{\Gamma}=\{1, x\}$ para as tensões de domínio e deslocamentos de contorno, respectivamente.

A danificação prepondera na região de aplicação da carga, onde os valores máximos obtidos com ambas as estratégias para determinação de dano são apresentados na Figura 6.36. Ressalta-se que se adotou $D_{c}=0,4$ quando da utilização do modelo simplificado de dano (calculado sobre resultados de uma análise linear).

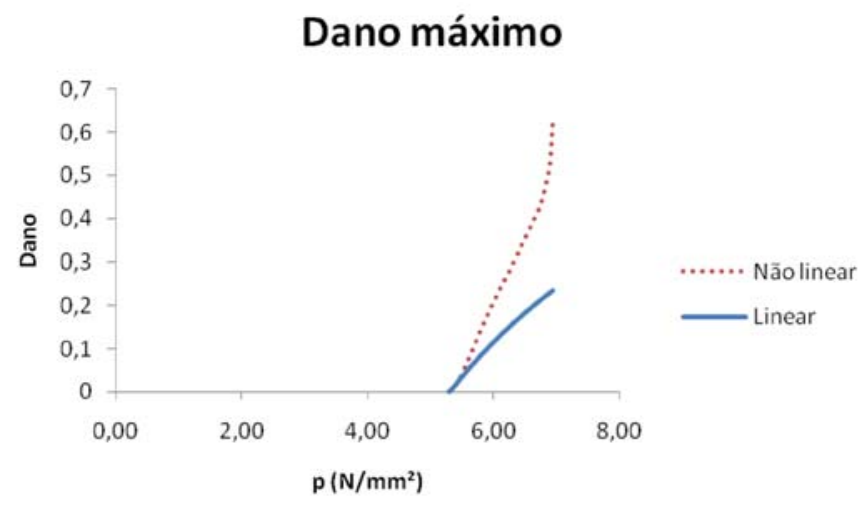

Figura 6.36 - Valores máximos de dano.

A distribuição do dano é apresentada na Figura 6.37 para diferentes valores da carga aplicada. 


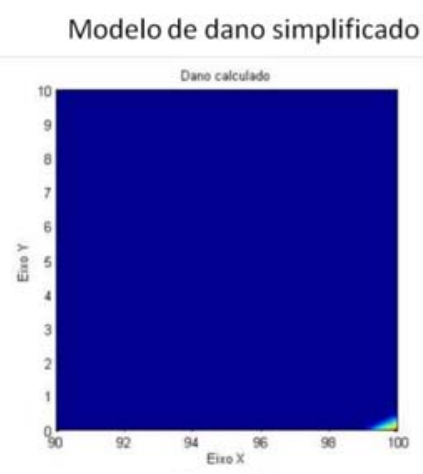

Modelo de dano acoplado (não linear)

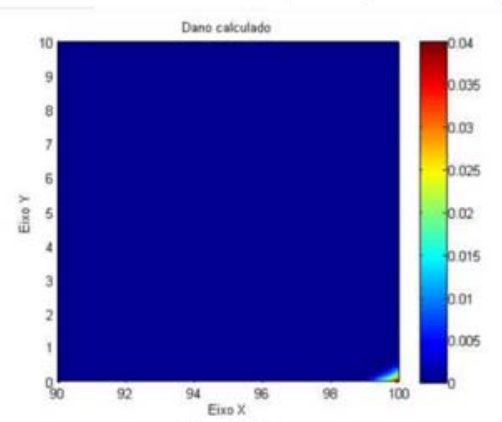

(a)
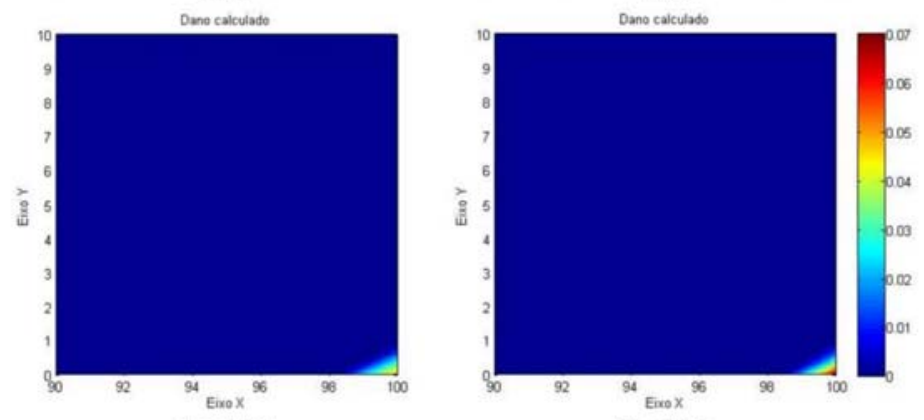

(b)
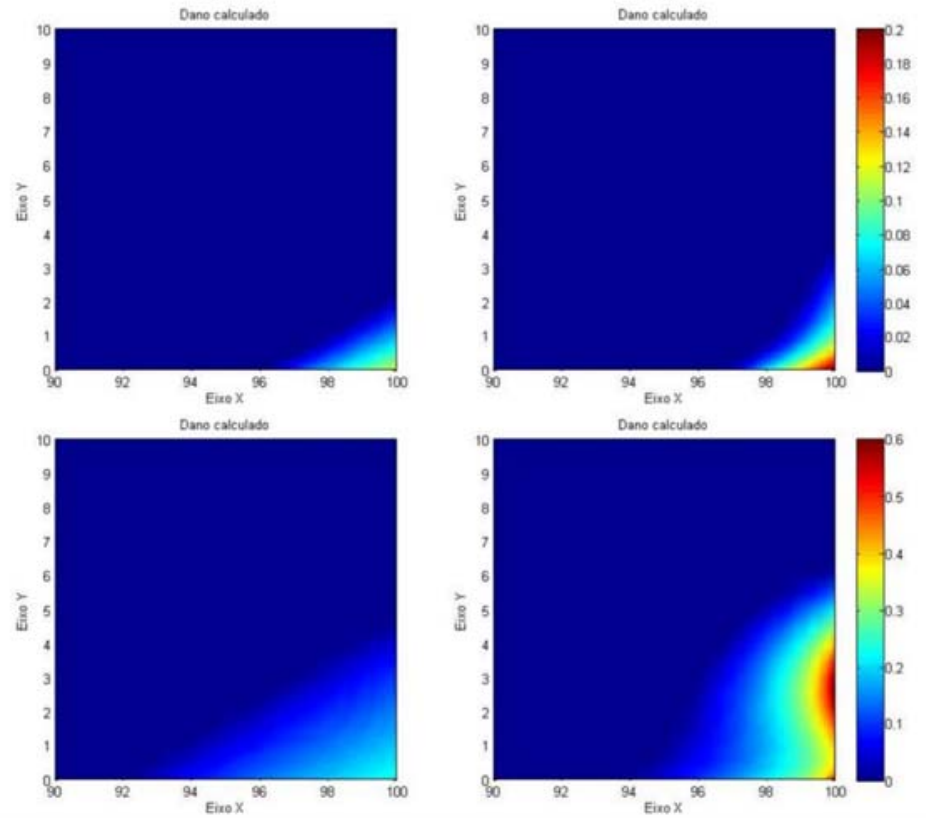

(c)

(d)

Figura 6.37 - Distribuição do dano para (a) $p=5,50 \mathrm{MPa}$, (b) $p=5,60 \mathrm{MPa}$, (c) $p=6,00 \mathrm{MPa}$ e (d) $p=$ $6,95 \mathrm{MPa}$.

Observa-se que até $p=5,60 \mathrm{MPa}(80,58 \%$ da carga máxima) os resultados obtidos pelo modelo de dano simplificado são bastante satisfatórios. Entretanto, praticamente em toda a análise pode-se observar que a caracterização da região danificada obtida com ambos os modelos é semelhante. 


\subsection{Teste de Hassanzadeh}

O teste experimental de Hassanzadeh, Silva (2006), corresponde à análise de um prisma de concreto simples com base quadrada de $70 \mathrm{~mm} \times 70 \mathrm{~mm}$ e altura de $60 \mathrm{~mm}$. Numa faixa de $4 \mathrm{~mm}$ a meia altura, a seção transversal do prisma é reduzida para $35 \mathrm{~mm} \times 35 \mathrm{~mm}$, no primeiro caso estudado, e $10 \mathrm{~mm} \times 10 \mathrm{~mm}$ no segundo caso. Naquela referência este ensaio numérico é realizado com a fixação do bordo inferior e aplicação de um deslocamento prescrito no bordo superior do prisma $(\bar{u})$, conforme exposto na Figura 6.38 .

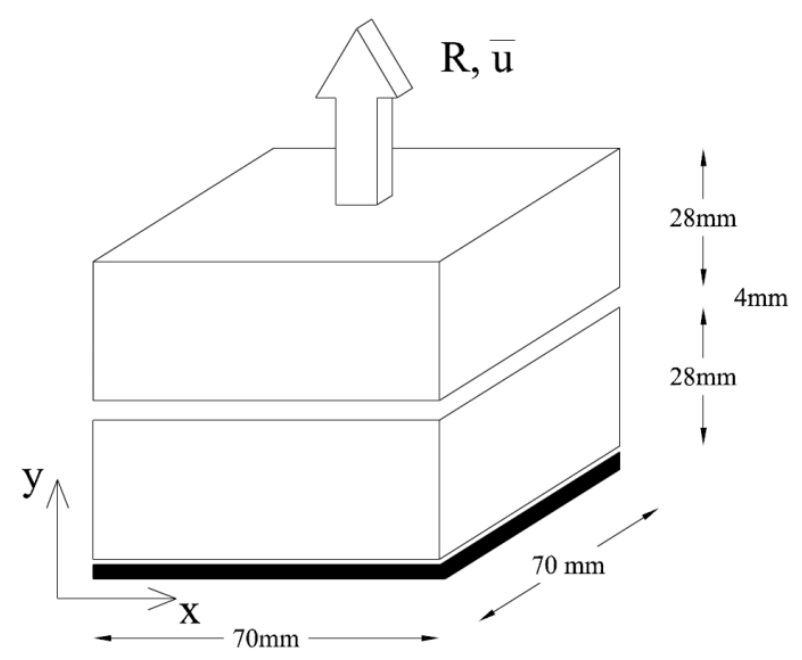

Figura 6.38 - Teste de Hassanzadeh. Fonte: Silva (2006).

Neste trabalho, ao invés de um deslocamento prescrito, foi aplicada uma força $(R)$ de forma monotônica, no bordo superior. Utiliza-se um concreto com os seguintes parâmetros físicos: $E=30 \mathrm{GPa}, v=0,2, n=3, k=0,00015 \mathrm{MPa}$ e $c=2,72$. Opta-se por não tirar partido da simetria do problema para que seja possível verificar se esta característica é reproduzida pelo modelo numérico. Considera-se um regime de estado plano de deformações (EPD).

Foram realizadas análises lineares e não lineares, apenas com a FHMT, a fim de confrontar o modelo de dano simplificado com a resposta obtida com o modelo totalmente acoplado. Ressalta-se que se adotou $D_{c}=0,4$ quando da utilização do modelo simplificado de dano.

Adotou-se $L_{2(\alpha)}^{\Omega}=\left\{1, x, x^{2}\right\}$ e $L_{2(\alpha)}^{\Gamma}=\{1, x\}$ como funções de enriquecimento para as tensões de domínio e deslocamentos de contorno, respectivamente. 
Define-se $\delta e$ como a razão entre os valores da energia de deformação obtidos com os modelos de dano simplificado $\left(U^{S I M P L}\right)$ e não linear $\left(U^{N L I N}\right)$, a fim de verificar o comportamento das respostas.

$\delta e=\frac{U^{S I M P L}}{U^{N L I N}}$

As primeiras análises foram realizadas sobre a geometria inicialmente proposta na referência, isto é, com a redução da seção transversal do prisma para $35 \mathrm{~mm} \times 35 \mathrm{~mm}$ (primeiro caso). Neste caso, a simulação não linear acoplada indicou um valor máximo da força aplicada no bordo superior de $\mathrm{R}=4756 \mathrm{~N}$. Até esse limite, a Figura 6.39 apresenta a resposta de $\delta e$ para os valores de R após o limite elástico.

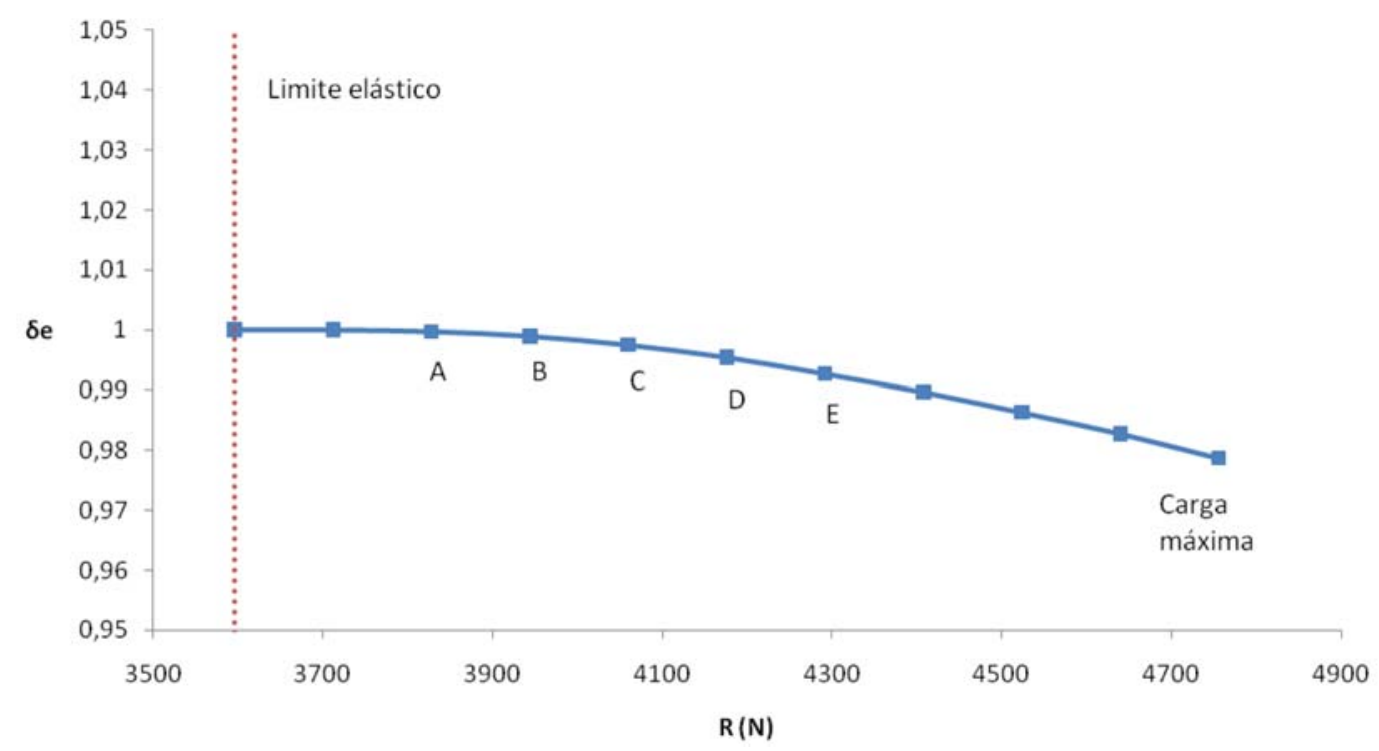

Figura 6.39 - Valores de $\delta e$ para a FHMT (primeiro caso).

Na Figura 6.39 são indicados pontos de controle (A, B, C, D e E) para fins de análise das respostas de dano no prisma (Figura 6.40). Para complementar a análise a Figura 6.41 apresenta os valores máximos de dano, obtidos com ambos os modelos citados. 
Resultados

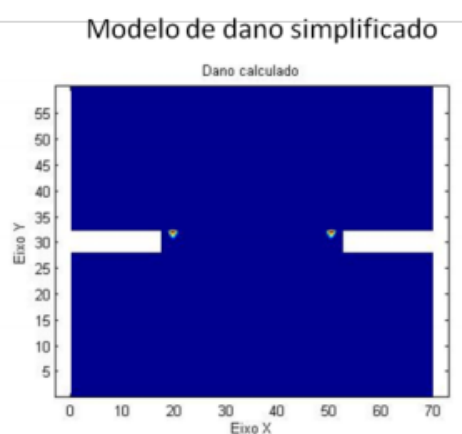

Modelo de dano acoplado (não linear)

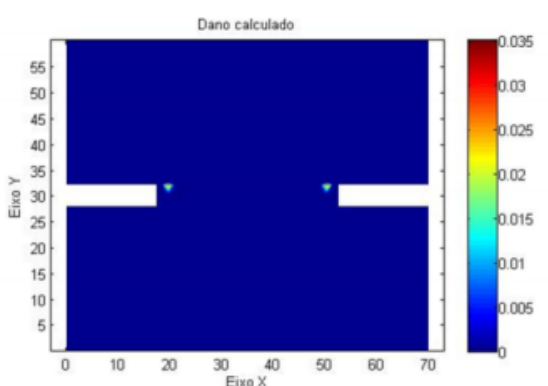

(A)
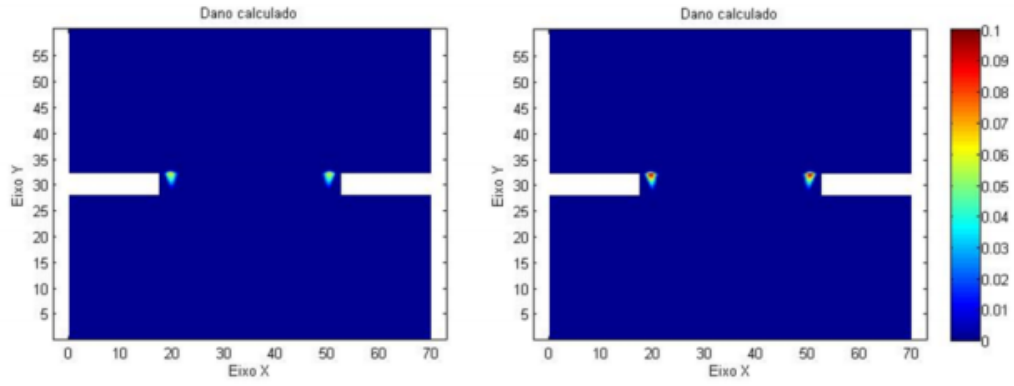

(B)
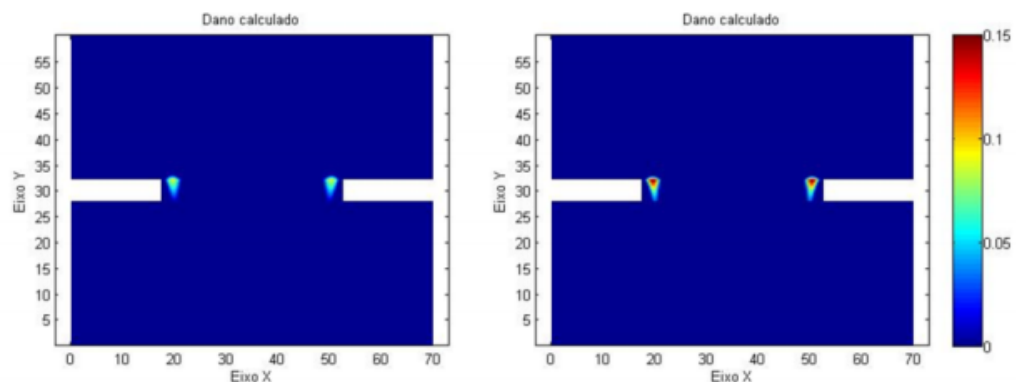

(C)
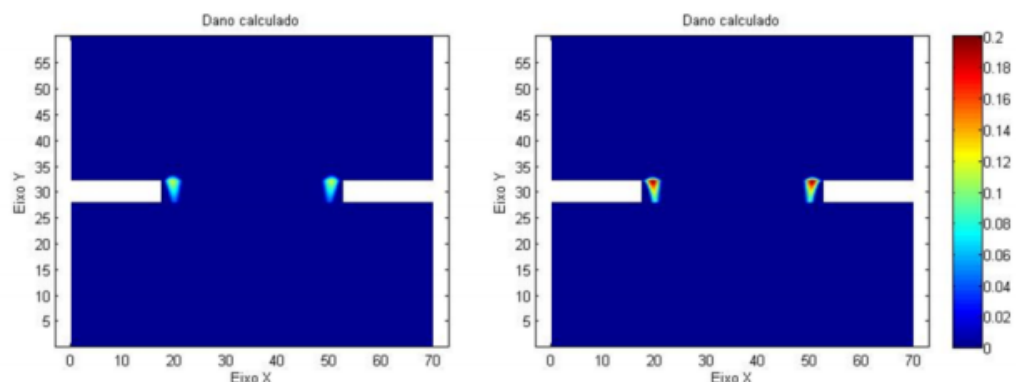

(D)
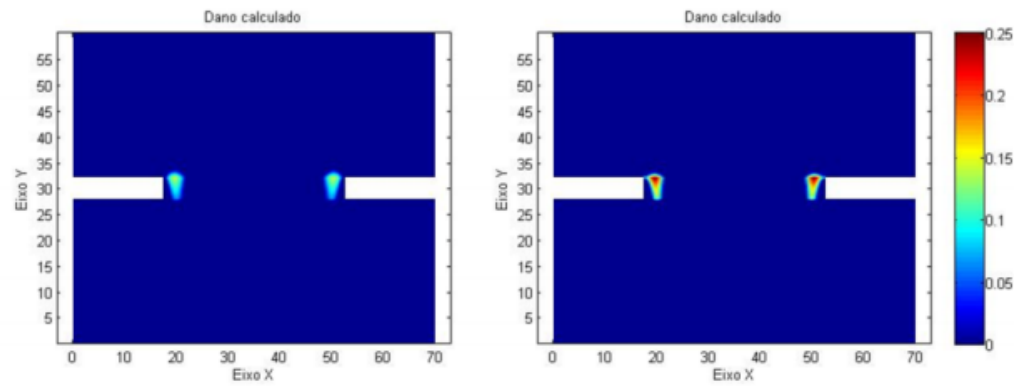

(E)

Figura 6.40 - Comparação da distribuição do dano nos pontos de controle (primeiro caso). 


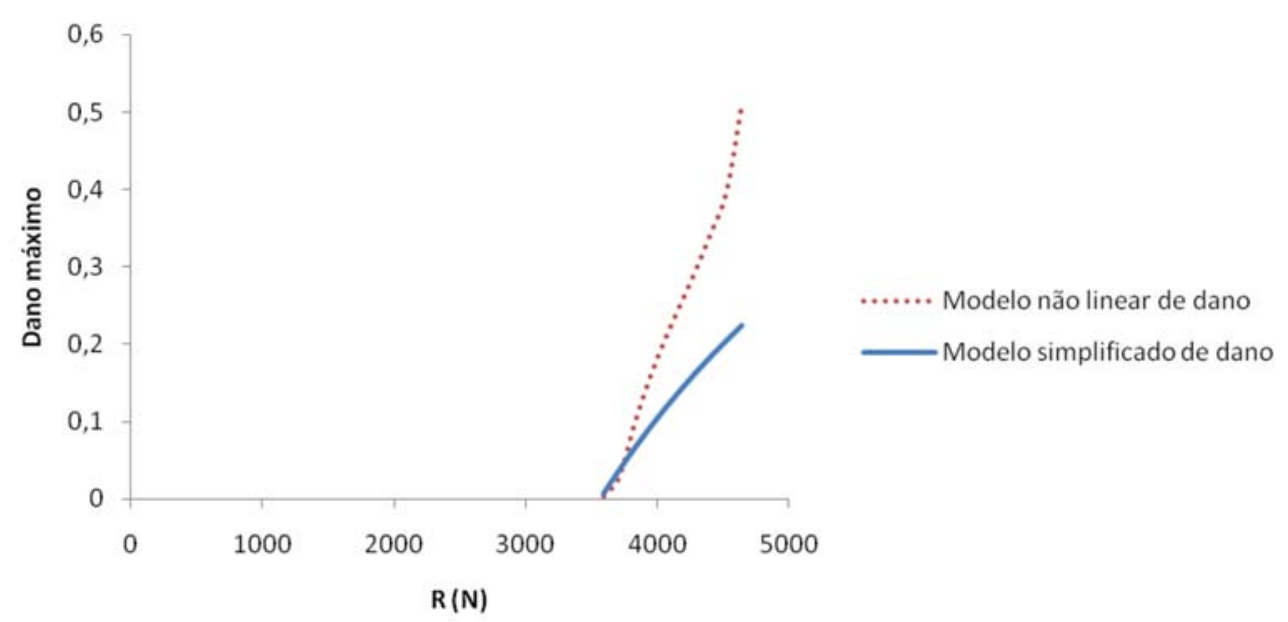

Figura 6.41 - Comparativo dos valores de dano máximo para o Teste de Hassanzadeh (primeiro caso).

Com os resultados anteriores pode-se afirmar que o modelo de dano simplificado apresentou comportamento satisfatório até o ponto de controle B $(82,93 \%$ da carga máxima aplicada), sendo seu comportamento admitido como razoável até o ponto de controle D. No ponto E pode-se observar que o valor máximo do dano calculado é pouco maior que $50 \%$ do valor máximo obtido com o modelo não linear. Contudo, em toda a análise a região danificada é praticamente a mesma para ambos os modelos de dano (Figura 6.42).

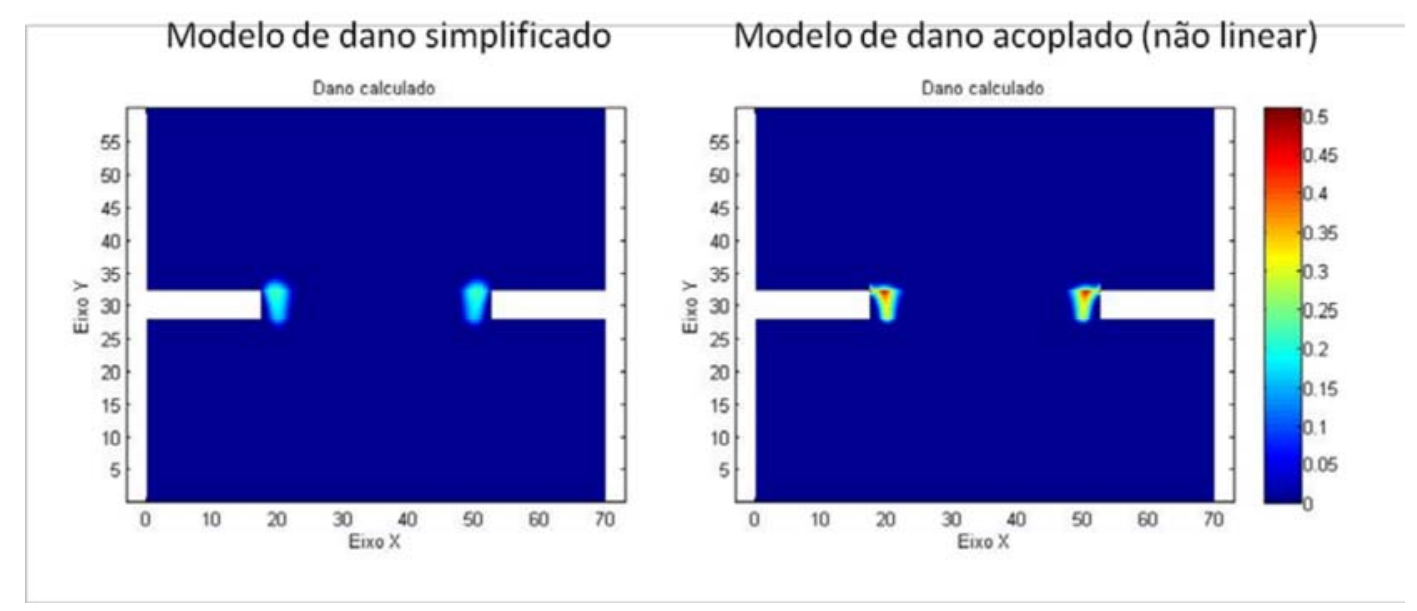

Figura 6.42 - Resposta de ambos os modelos de dano para a carga máxima $(\mathrm{R}=4756 \mathrm{~N})$.

As análises a seguir foram realizadas com a redução da seção transversal do prisma para $10 \mathrm{~mm} \times 10 \mathrm{~mm}$ (segundo caso). Neste caso, com a utilização do modelo não linear, observou-se que o valor máximo da força máxima aplicada no bordo superior foi $\mathrm{R}=$ 5280 N. A Figura 6.43 apresenta a resposta de $\delta e$ para os valores de R após o limite elástico. 
Resultados

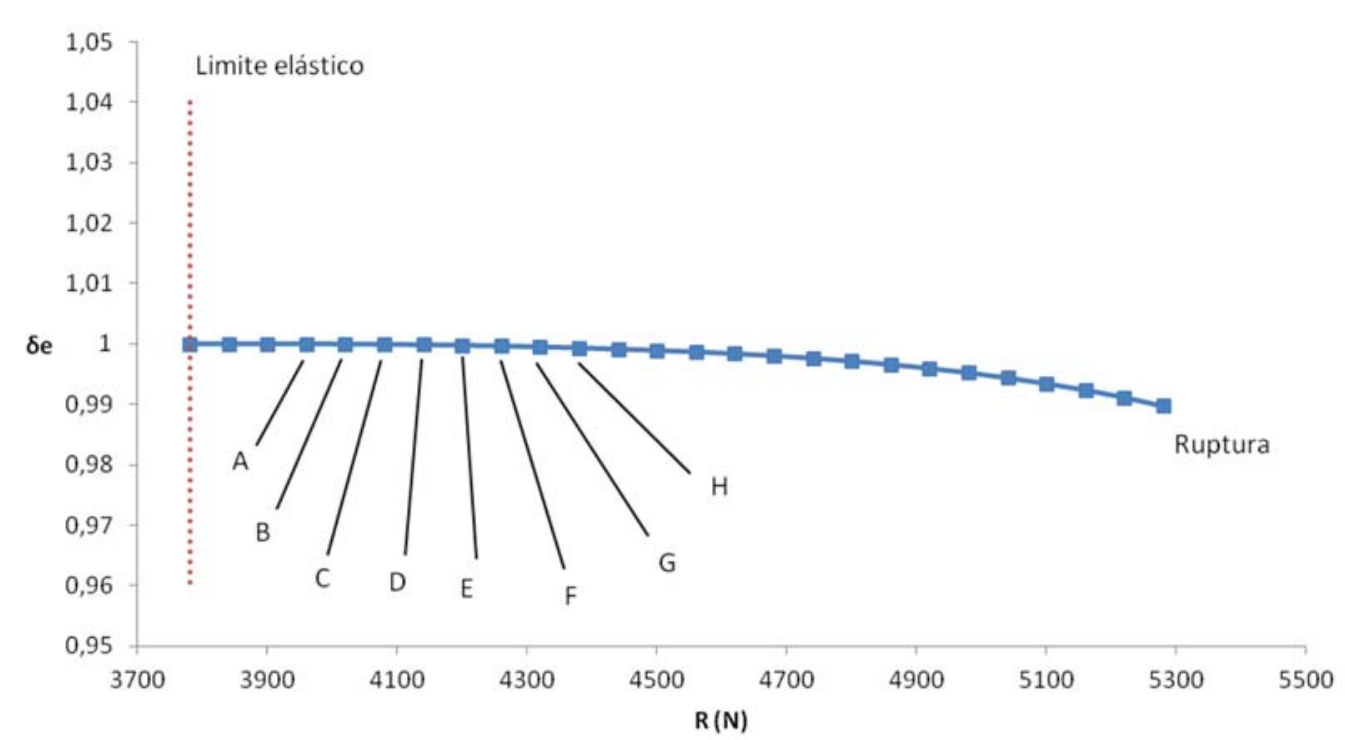

Figura 6.43 - Valores de $\delta e$ para a FHMT (segundo caso).

Conforme indicado na Figura 6.43 foram adotados pontos de controle (A, B, C, D, E, $\mathrm{F}, \mathrm{G}$ e $\mathrm{H}$ ) a fim de analisar as respostas de dano no prisma. Para complementar a análise dos resultados nos pontos de controle, a Figura 6.44 apresenta um comparativo dos valores máximos de dano obtidos com os modelos não linear e simplificado.

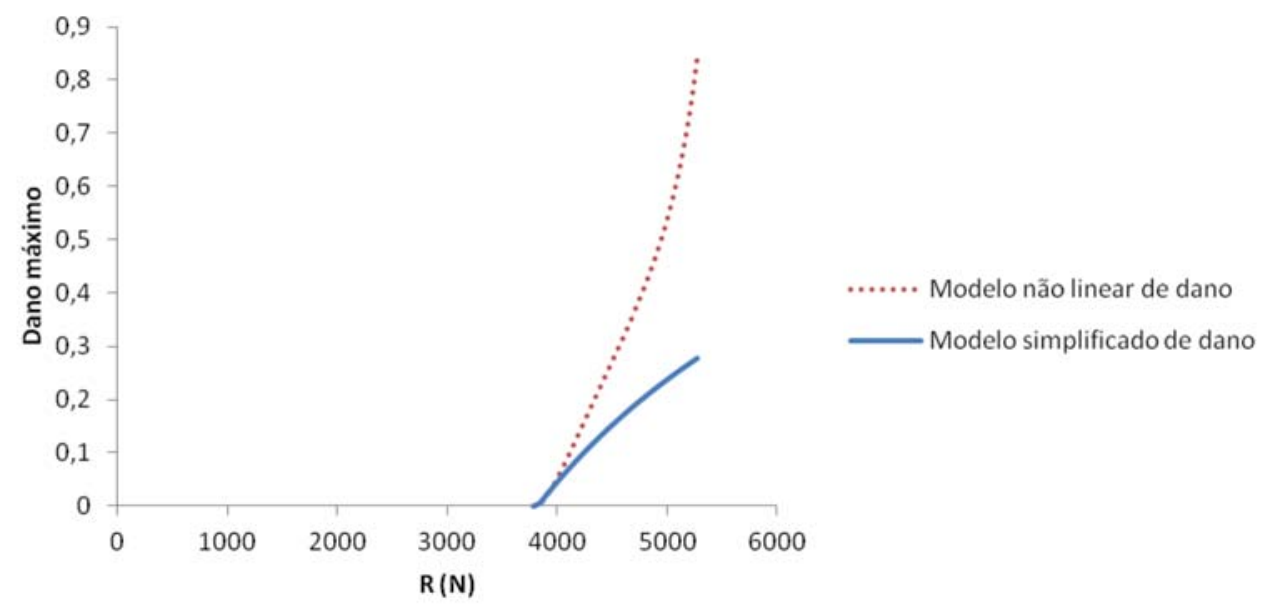

Figura 6.44 - Comparativo dos valores de dano máximo para o Teste de Hassanzadeh (segundo caso).

Para os três primeiros pontos de controle (Figura 6.45) observou-se que o modelo de dano simplificado acompanha bem os resultados do modelo não linear. 
Modelo de dano simplificado

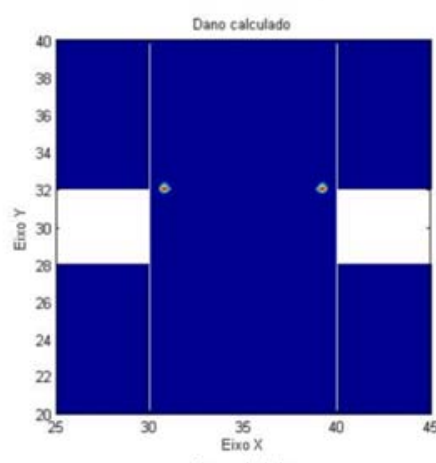

Dano calculado

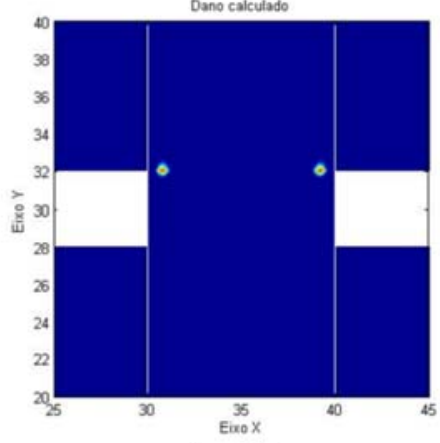

Dano calculado

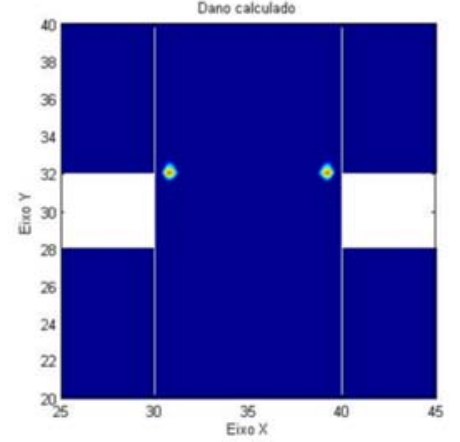

Modelo de dano acoplado (não linear)

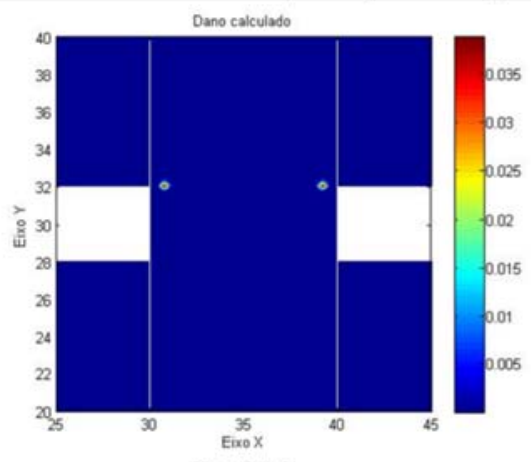

Dano calculado

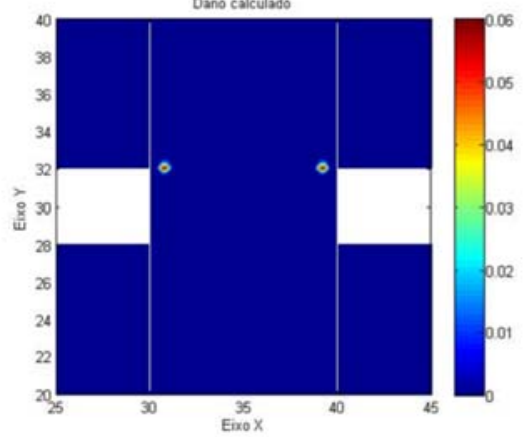

Dano calculado

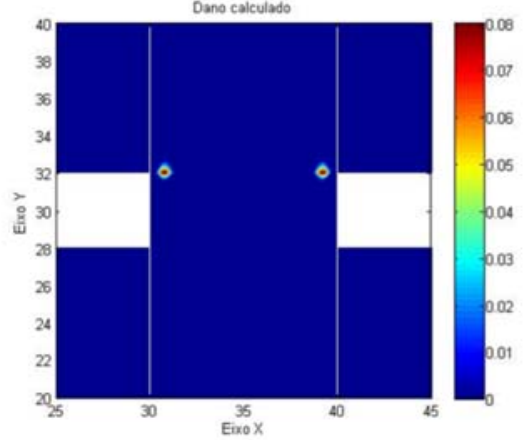

(A)

(B)

(C)

Figura 6.45 - Comparação da distribuição do dano nos pontos de controle A, B e C (segundo caso). 
Resultados

Modelo de dano simplificado

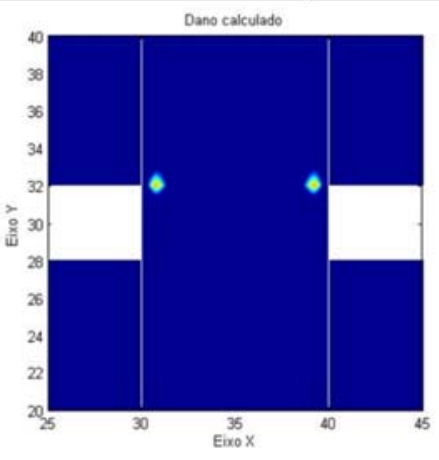

Dano calculado

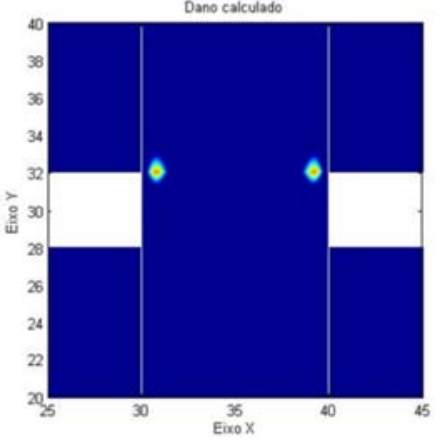

Dano calculado

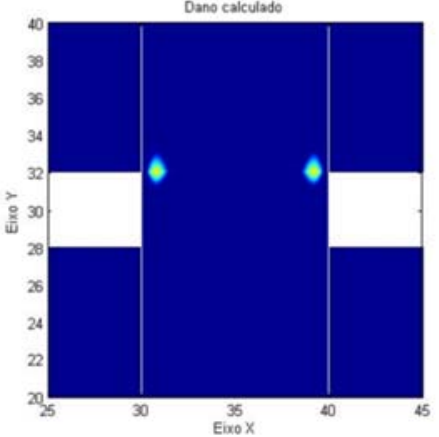

Modelo de dano acoplado (não linear)

Dano calculado

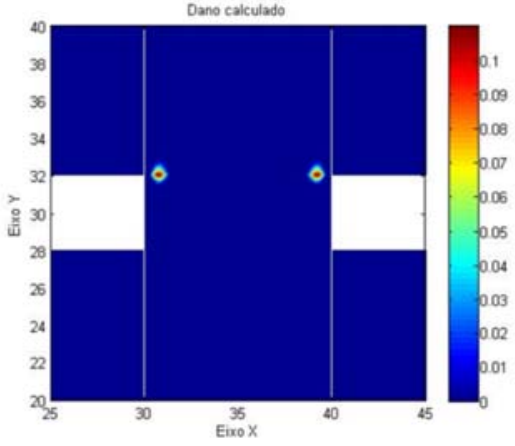

Dano calculado
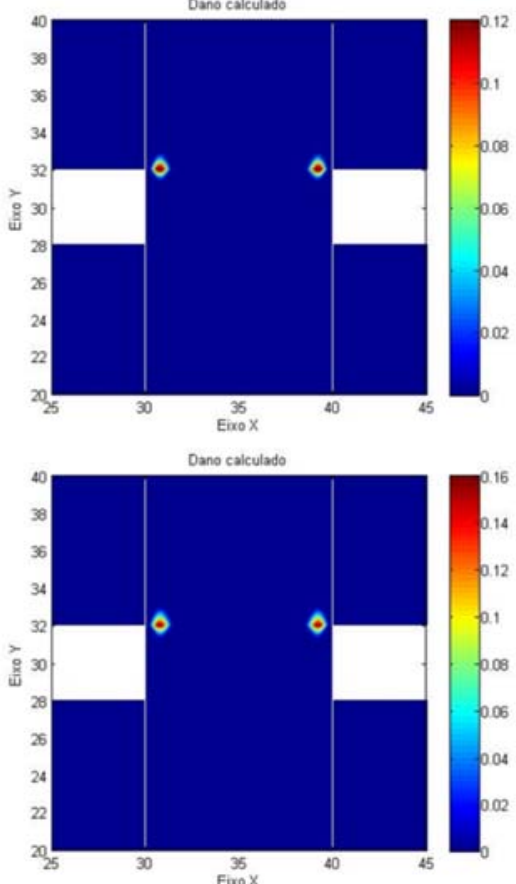

(D)

(E)

(F)

Figura 6.46 - Comparação distribuição do dano nos pontos de controle D, E e F (segundo caso).

Conforme se apresenta na Figura 6.46, nos pontos de controle D, E e F $(80,68 \%$ da carga máxima aplicada) o resultado também é satisfatório. Já nos pontos de controle $\mathrm{G}$ e $\mathrm{H}$ (Figura 6.47) os valores de dano obtidos com o modelo simplificado começam a divergir dos valores provenientes do modelo não linear. Entretanto, observa-se que a região danificada é semelhante em ambos os modelos. 


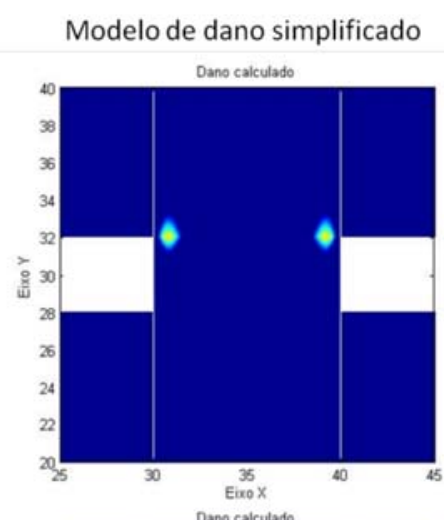

Modelo de dano acoplado (não linear)
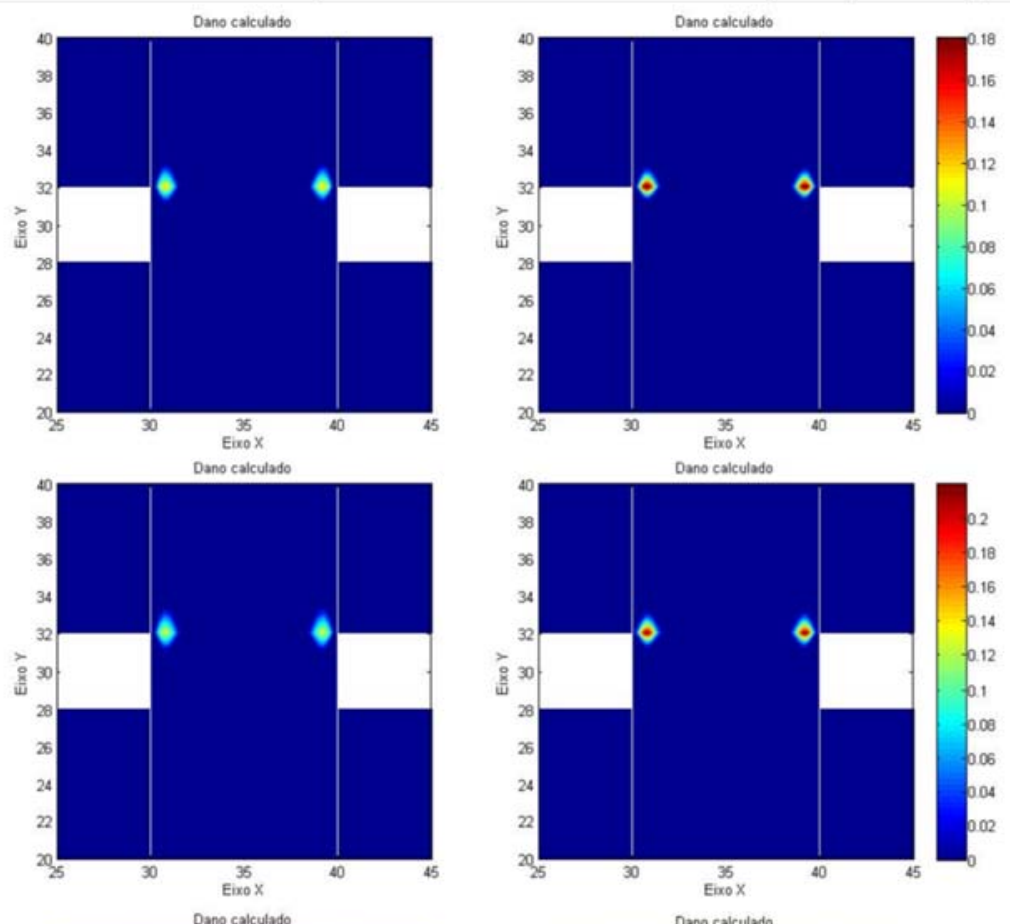

(G)

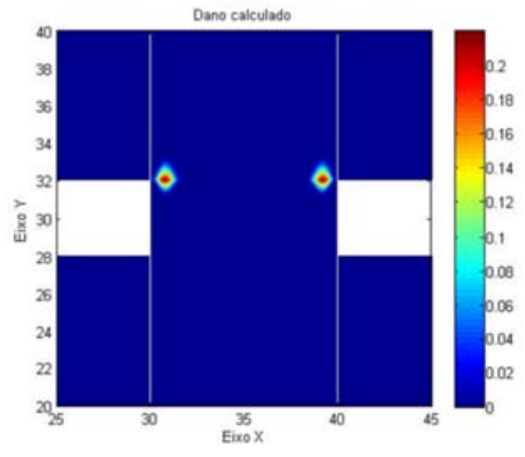

(H)
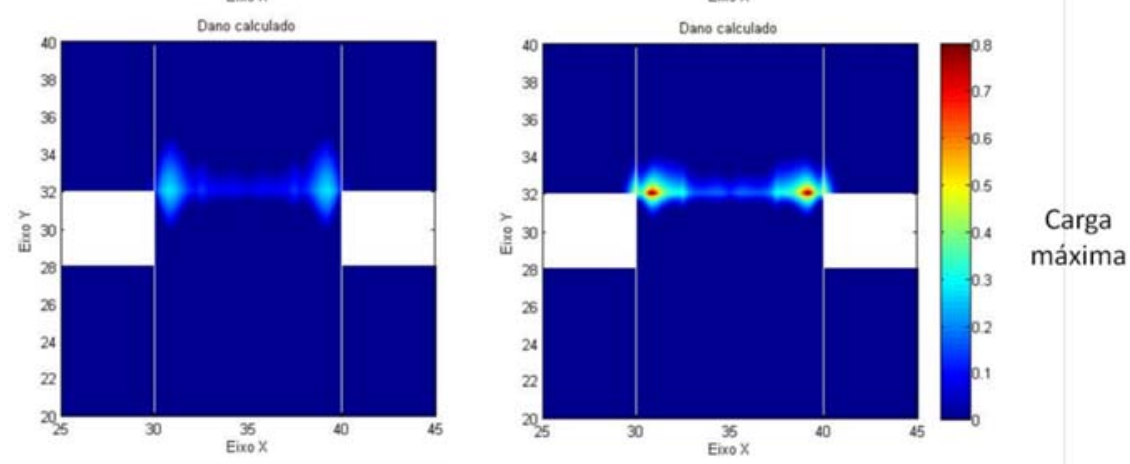

Figura 6.47 - Comparação distribuição do dano nos pontos de controle G e H, e para a carga máxima (segundo caso).

Analisando dois casos do Teste de Hassanzadeh viu-se que a geometria do modelo provocou localização da distribuição de dano, o que favoreceu a aproximação com o modelo simplificado. 


\section{Conclusões}

Um primeiro objetivo deste trabalho foi contribuir para o estudo de formulações não convencionais para o MEFG, tendo como foco a FHMT e a $\operatorname{FM}(u-\sigma)$. Primeiramente, verificou-se a capacidade de aproximação das formulações não convencionais em estudo num exemplo consagrado da literatura, conhecido como teste de distorção. Neste exemplo observou-se que o emprego de funções de enriquecimento permitiu eliminar a sensibilidade à distorção imposta nos elementos. No segundo exemplo buscou-se analisar a convergência das formulações não convencionais, com e sem enriquecimento, considerando-se um problema de elasticidade plana. O problema de uma chapa tracionada evidenciou a boa capacidade de aproximação e convergência para a solução de referência adotada tanto da FHMT quanto da $\mathrm{FM}(\mathrm{u}-\sigma)$.

Como as formulações não convencionais aproximam o campo de tensões de forma direta, verificou-se a capacidade de aproximação destas num exemplo com singularidade. Este tratou da mesma chapa do exemplo anterior, acrescendo-se uma fissura discreta na sua região central. Confrontaram-se as respostas da FHMT e FM(u- $\sigma)$ combinadas com o MEFG, mostrando que neste tipo de exemplo as formulações recuperam de forma satisfatória a solução de referência.

Um segundo objetivo do trabalho foi explorar a boa capacidade de aproximação das tensões para a análise de problemas que envolvam danificação. Nesse sentido foi proposta uma estratégia simplificada, que consiste em estimar a intensidade do dano utilizando-se apenas os resultados de uma análise linear. Os resultados dessa estratégia foram confrontados com os obtidos por uma análise não linear incremental-iterativa.

Num primeiro exemplo, simulou-se um caso de tração simples, com o intuito de validar a análise não linear de dano e iniciar a comparação entre a abordagem completa e a estratégia simplificada. Observou-se que os valores de dano obtidos pelo modelo simplificado podem ser considerados satisfatórios frente aos valores obtidos com o modelo totalmente acoplado (não linear) somente numa estreita faixa de tensão logo após o aparecimento do dano. Entretanto, esta condição era esperada, dado que a danificação ocorreu em todo o elemento estrutural.

Nos exemplos seguintes, procurou-se avaliar o modelo de dano simplificado em problemas com danificação em regiões localizadas da estrutura. O exemplo de uma chapa 
com tração não uniforme induz danificação localizada em uma faixa de sua borda adjacente à aplicação do carregamento. Neste exemplo pôde-se observar que a aproximação dos resultados da estratégia simplificada com os do modelo não linear foi satisfatória para até $80 \%$ da carga máxima aplicada.

No exemplo seguinte, realizou-se a simulação de um prisma tracionado, com seção central reduzida. Neste exemplo constatou-se que o modelo de dano simplificado apresentou resultados satisfatórios para valores da carga aplicada entre $80 \%$ e $83 \%$ da carga máxima. Nestes exemplos também se observou que a região danificada obtida pelo modelo simplificado se assemelha a dada com o modelo não linear de dano, independente da carga aplicada.

Desta forma, pode-se concluir que a estratégia simplificada é representativa nos casos de dano localizado em pequenas regiões de modo que se possa dispensar os passos iterativos associados à redistribuição das tensões. Entretanto, mesmo nos casos de dano difuso ainda é possível realizar uma análise qualitativa com o modelo de dano simplificado, visto que a região danificada é semelhante àquela obtida com o modelo totalmente acoplado (não linear). 


\section{Referências bibliográficas}

ARRUDA, M. R. T. Static and dynamic analysis of concrete structures using damage mechanics. Tese (Doutorado) - Instituto Superior Técnico, Universidade Técnica de Lisboa, Lisboa, 2011. 258 p.

BATHE, K. J. Finite element procedures. 2nd ed. New Jersey: Prentice-Hall. 1996.

COMI , C; PEREGO, U. Numerical aspects of nonlocal damage analyses. Revue Européenne des Éléments Finis, v. 10, 2001, p.227-242.

DUARTE, C. A. The hp-cloud method. Tese (Doutorado) - The University of Texas at Austin, Austin, 1996.

DUARTE, C. A.; KIM, D. J. Analysis and applications of a generalized finite element method with global-local enrichment functions. Computer Methods in Applied Mechanics and Engineering, v. 197, 2008, p. 487-504.

GÓIS, W. Elementos finitos híbridos e híbrido-mistos de tensão com enriquecimento nodal. Tese (Doutorado) - Escola de Engenharia de São Carlos, Universidade de São Paulo, São Carlos, 2009. 223 p.

LEMAITRE J.; DESMORAT, R. Engineering Damage Mechanics. Springer-Verlag. BerlinHeidelberg. 2005.

PROENÇA, S. P. B. Introdução aos métodos numéricos. Notas de aula - Escola de Engenharia de São Carlos, Universidade de São Paulo, São Carlos, 2007. 145p.

PROENÇA, S. P. B. Mecânica do dano. Notas de aula - Escola de Engenharia de São Carlos, Universidade de São Paulo, São Carlos, 2008. 51p.

PROENÇA, S. P. B. Método dos Elementos Finitos Generalizados. Notas de aula - Escola de Engenharia de São Carlos, Universidade de São Paulo, São Carlos, 2010. 54p. 
SAS (Swanson Analysis Systems Inc.). ANSYS Resference Manual (version 12). 2009.

SILVA, M. C. O. M. Modelos de Dano em Elementos Finitos Híbridos e Mistos. Tese (Doutorado) - Instituto Superior Técnico, Universidade Técnica de Lisboa, Lisboa, 2006. 319 p.

STROUBOULIS, T.; BABUŠKA, I.; COOPS, K. The design and analysis of the Generalized Finite Element Method. Computer Methods in Applied Mechanics and Engineering, v. 181,2000 , p. 43-69.

SZABÓ, B.; BABUŠKA, I. Finite element analysis. John Wiley \& Sons. 1991.

TIMOSHENKO, S. P.; GOODIER, J. N. Teoria da elasticidade. Guanabara Dois S. A. 1980.

TORRES, I. F. R. Desenvolvimento e aplicação do método dos elementos finitos generalizados em análise tridimensional não-linear de sólidos. Tese (Doutorado). Escola de Engenharia de São Carlos, Universidade de São Paulo, São Carlos, 2003. 221p.

VALLIAPAN, S. Continuum mechanics: fundamentals. A. A. Balkema. 1981.

XU, Z. Applied elasticity. John Wiley \& Sons. 1992.

ZIENKIEWICZ, O. C.; TAYLOR, R. L. The finite element method - Volume 1: the basis. $5^{\text {th }}$ ed. Oxford: Butterworth-Heinemann. 2000. 


\section{APÊNDICE A - Modelos discretos com enriquecimento nodal}

Aqui se destaca a formalização das formulações Híbrido-Mista de Tensão e Mista u- $\sigma$ com enriquecimento nodal polinomial para as redes de cobertura compostas por elementos finitos quadrangulares convencionais de quatro nós e elementos lineares de contorno, sendo este último apenas para a FHMT.

As aplicações dos enriquecimentos nodais às formulações não convencionais utilizadas caracterizam-nas como formas alternativas do Método dos Elementos Finitos Generalizados (MEFG). Neste trabalho a FHMT e a FM(u- $\sigma)$ têm seus graus de liberdade vinculados aos nós, tornando possível a utilização da metodologia clássica de enriquecimento do Método das Nuvens $h p$.

\section{A.1 Modelo discreto da FHMT com enriquecimento nodal}

Este item está fortemente ligado ao trabalho de Góis (2009), do qual a ferramenta computacional, que realiza análises lineares com a FHMT, foi aproveitada.

Considere-se um domínio $\boldsymbol{\Omega}$ composto por $\boldsymbol{n}_{\boldsymbol{\Omega}}$ elementos finitos de domínio, onde $\boldsymbol{\Omega}_{\boldsymbol{e}}\left(\boldsymbol{e}=\mathbf{1}, \ldots, \boldsymbol{n}_{\boldsymbol{\Omega}}\right)$ são os domínios de cada um dos $\boldsymbol{n}_{\boldsymbol{\Omega}}$ elementos. Também são definidos $\boldsymbol{n}_{\boldsymbol{\Gamma}_{\boldsymbol{t}}}$ elementos no contorno de Neumann $\boldsymbol{\Gamma}_{\boldsymbol{t}_{\boldsymbol{\Gamma}_{\boldsymbol{t}}}}\left(\boldsymbol{e}_{\boldsymbol{\Gamma}_{\boldsymbol{t}}}=\mathbf{1}, \ldots, \boldsymbol{n}_{\boldsymbol{\Gamma}_{\boldsymbol{t}}}\right)$ e $\boldsymbol{n}_{\boldsymbol{\Gamma}_{\boldsymbol{i}}}$ elementos no contorno entre elementos de domínio $\boldsymbol{\Gamma}_{\boldsymbol{i}_{\boldsymbol{\Gamma}_{\boldsymbol{i}}}}\left(\boldsymbol{e}_{\boldsymbol{\Gamma}_{\boldsymbol{i}}}=\mathbf{1}, \ldots, \boldsymbol{n}_{\boldsymbol{\Gamma}_{i}}\right)$. Também se deve considerar que no contorno de Dirichlet $\boldsymbol{\Gamma}_{\boldsymbol{u}_{\boldsymbol{e}_{\boldsymbol{u}}}}$ existem $\boldsymbol{n} \boldsymbol{e}_{\boldsymbol{\Gamma}_{\boldsymbol{u}}}=1, \ldots, \boldsymbol{n}_{\boldsymbol{\Gamma}_{\boldsymbol{u}}}$ elementos com deslocamentos prescritos.

Os vetores $\boldsymbol{s}_{\Omega_{e}}, \quad \boldsymbol{q}_{\Omega_{e}}, \quad \boldsymbol{q}_{\boldsymbol{\Gamma}_{\Gamma_{t}}}$ e $\boldsymbol{q}_{\boldsymbol{\Gamma}_{\boldsymbol{\Gamma}_{i}}}$ armazenam os parâmetros generalizados correspondentes às aproximações locais para as tensões e deslocamentos nos elementos de domínio, e deslocamentos dos elementos dos contornos de Neumann e internos, respectivamente. Os vetores $\boldsymbol{s}_{\Omega}, \boldsymbol{q}_{\Omega}, \boldsymbol{q}_{\boldsymbol{\Gamma}_{t}}$ e $\boldsymbol{q}_{\boldsymbol{\Gamma}_{i}}$, referentes às aproximações globais, foram definidos no capítulo 3 .

Os parâmetros locais e globais se relacionam por matrizes Booleanas $\langle\boldsymbol{B}(\cdot)\rangle$, da seguinte forma:

$\boldsymbol{s}_{\Omega_{e}}=\left\langle B\left(F_{e}\right)\right\rangle s_{\Omega}$ 
$\boldsymbol{q}_{\Omega_{e}}=\left\langle B\left(A_{e}\right)\right\rangle q_{\Omega}$

$q_{\Gamma_{\Gamma_{t}}}=\left\langle B\left(A_{\Gamma_{\Gamma_{t}}}\right)\right\rangle q_{\Gamma_{t}}$

$\boldsymbol{q}_{\Gamma_{\Gamma_{i}}}=\left\langle B\left(A_{\Gamma_{e_{i}}}\right)\right\rangle q_{\Gamma_{i}}$

As matrizes $\left\langle\boldsymbol{B}\left(\boldsymbol{F}_{\boldsymbol{e}}\right)\right\rangle$ e $\left\langle\boldsymbol{B}\left(\boldsymbol{A}_{\boldsymbol{e}}\right)\right\rangle$ coletam os parâmetros generalizados dos elementos de domínio $\boldsymbol{\Omega}_{\boldsymbol{e}}\left(\boldsymbol{e}=\mathbf{1}, \ldots, \boldsymbol{n}_{\boldsymbol{\Omega}}\right)$, e as matrizes $\left\langle\boldsymbol{B}\left(\boldsymbol{A}_{\boldsymbol{\Gamma}_{\boldsymbol{\Gamma}_{\boldsymbol{t}}}}\right)\right\rangle$ e $\left\langle\boldsymbol{B}\left(\boldsymbol{A}_{\boldsymbol{\Gamma}_{\boldsymbol{\Gamma}_{\Gamma_{i}}}}\right)\right\rangle$ coletam os parâmetros generalizados dos elementos dos contornos $\boldsymbol{\Gamma}_{\boldsymbol{t}_{\boldsymbol{\Gamma}_{\boldsymbol{t}}}}\left(\boldsymbol{e}_{\boldsymbol{\Gamma}_{\boldsymbol{t}}}=1, \ldots, \boldsymbol{n}_{\boldsymbol{\Gamma}_{t}}\right)$ e $\boldsymbol{\Gamma}_{\boldsymbol{i}_{\boldsymbol{\Gamma}_{i}}}\left(\boldsymbol{e}_{\boldsymbol{\Gamma}_{i}}=1, \ldots, \boldsymbol{n}_{\boldsymbol{\Gamma}_{i}}\right)$, respectivamente.

As aproximações locais dos campos de tensão e deslocamento de domínio, deslocamento no contorno de Neumann e deslocamento no contorno interno são dadas da eq.(A.5) a eq.(A.8):

$\widehat{\boldsymbol{\sigma}}_{e}=\boldsymbol{S}_{\boldsymbol{\Omega}_{e}} \boldsymbol{S}_{\boldsymbol{\Omega}_{e}}$

$\widehat{\boldsymbol{u}}_{e}=\boldsymbol{U}_{\Omega_{e}} \boldsymbol{q}_{\Omega_{e}}$

$\widehat{\boldsymbol{u}}_{\Gamma_{t}}=U_{\Gamma_{t_{\Gamma_{t}}}} q_{\Gamma_{e_{\Gamma_{t}}}}$

$u_{\Gamma_{i}}=U_{\Gamma_{t_{\Gamma_{i}}}} q_{\Gamma_{e_{\Gamma_{i}}}}$

sendo $\boldsymbol{S}_{\Omega_{e}}, \boldsymbol{U}_{\Omega_{e}}, \boldsymbol{U}_{\boldsymbol{\Gamma}_{\boldsymbol{t}_{\boldsymbol{\Gamma}_{\boldsymbol{t}}}}}$ e $\boldsymbol{U}_{\boldsymbol{\Gamma}_{\boldsymbol{i}_{\boldsymbol{\Gamma}_{\boldsymbol{i}}}}}$ as matrizes das funções de interpolação dos campos de tensão e deslocamento no domínio, deslocamento no contorno de Neumann e deslocamento no contorno interno, respectivamente.

Com a contribuição de cada elemento finito, no domínio e no contorno, montam-se as matrizes que compõem o sistema resolvente da FHMT, dado pela eq.(3.31). Assim, a matriz de flexibilidade generalizada $(\boldsymbol{F})$, por exemplo, é obtida com o endereçamento dado pela 
matriz Booleana $\left\langle\boldsymbol{B}\left(\boldsymbol{F}_{\boldsymbol{e}}\right)\right\rangle$ a matriz local $\boldsymbol{F}_{\boldsymbol{e}}$, conforme apresenta a eq.(A.9). De maneira semelhante, as demais matrizes e vetores que compõem o sistema da FHMT são obtidos, conforme exposto da eq.(A.10) a eq.(A.15):

$F=\sum_{e=1}^{n_{\Omega}}\left\langle B\left(F_{e}\right)\right\rangle^{\mathrm{T}} F_{e}\left\langle B\left(F_{e}\right)\right\rangle$

$\boldsymbol{A}_{\Omega}=\sum_{\boldsymbol{e}=1}^{n_{\Omega}}\left\langle B\left(F_{e}\right)\right\rangle^{\mathrm{T}} \boldsymbol{A}_{\Omega_{e}}\left\langle B\left(\boldsymbol{A}_{e}\right)\right\rangle$

$A_{\Gamma_{t}}=\sum_{e_{\Gamma_{t}}=1}^{n_{\Gamma_{t}}}\left\langle B\left(F_{e}\right)\right\rangle^{\mathbf{T}} A_{\Gamma_{t_{e}}}\left\langle B\left(A_{\Gamma_{e_{\Gamma_{t}}}}\right)\right\rangle$

$A_{\Gamma_{i}}=\sum_{e_{\Gamma_{i}}=1}^{n_{\Gamma_{i}}}\left\langle B\left(F_{e}\right)\right\rangle^{\mathrm{T}} A_{\Gamma_{i_{e}}}\left\langle B\left(A_{\Gamma_{\Gamma_{\Gamma_{i}}}}\right)\right\rangle$

$e_{\Gamma_{u}}=\sum_{n e_{\Gamma_{u}}=1}^{n_{\Gamma_{u}}}\left\langle B\left(F_{e}\right)\right\rangle^{\mathrm{T}} e_{\Gamma_{u_{n e_{\Gamma_{u}}}}}$

$\boldsymbol{Q}_{\Omega}=\sum_{e=1}^{n_{\Omega}}\left\langle B\left(A_{e}\right)\right\rangle^{\mathrm{T}} \boldsymbol{Q}_{\Omega_{e}}$

$\boldsymbol{Q}_{\Gamma_{t}}=\sum_{e_{\Gamma_{t}}=1}^{n_{\Gamma_{t}}}\left\langle B\left(A_{\Gamma_{\Gamma_{\Gamma_{t}}}}\right)\right\rangle^{\mathrm{T}} \boldsymbol{Q}_{\Gamma_{e_{\Gamma_{t}}}}$

onde

$F_{e}=\int_{\Omega} S_{\Omega_{\mathrm{e}}}^{\mathrm{T}} f S_{\Omega_{\mathrm{e}}} d \Omega$ 
$A_{\Omega_{\mathrm{e}}}=\int_{\Omega}\left(\mathrm{L} S_{\Omega_{\mathrm{e}}}\right)^{\mathrm{T}} U_{\Omega_{\mathrm{e}}} d \Omega$

$A_{\Gamma_{t_{e_{t}}}}=\int_{\Gamma_{\mathrm{t}}}\left(\mathrm{N} S_{\Omega_{\mathrm{e}}}\right)^{\mathrm{T}} U_{\Gamma_{t_{e_{\Gamma_{t}}}}} d \Gamma$

$A_{\Gamma_{i_{\Gamma_{i}}}}=-\int_{\Gamma_{i}}\left(\mathrm{~N} S_{\Omega_{\mathrm{e}}}\right)^{\mathrm{T}} U_{\Gamma_{i_{\Gamma_{\Gamma_{i}}}}} d \Gamma$

$e_{\Gamma_{u_{n e_{\Gamma_{u}}}}}=\int_{\Gamma_{\mathrm{u}}}\left(\mathrm{N} S_{\Omega_{\mathrm{e}}}\right)^{\mathrm{T}} \bar{u} d \Gamma$

$Q_{\Omega_{\mathrm{e}}}=\int_{\Omega} U_{\Omega_{\mathrm{e}}}^{\mathrm{T}} \bar{b} d \Omega$

$Q_{\Gamma_{\boldsymbol{e}_{t}}}=\int_{\Gamma_{t}} U_{\Gamma_{t_{e}}}^{\mathrm{T}} \bar{t} d \Gamma$

Neste trabalho é utilizado apenas o elemento finito híbrido-misto de tensão quadrilateral de quatro nós, apresentado na Figura A.1. Ainda nesta figura, indica-se o posicionamento dos elementos lineares de contorno e aponta-se o mapeamento do elemento isoparamétrico, de coordenadas $\xi \eta$, para uma forma quadrilateral qualquer no sistema cartesiano $x y$. 


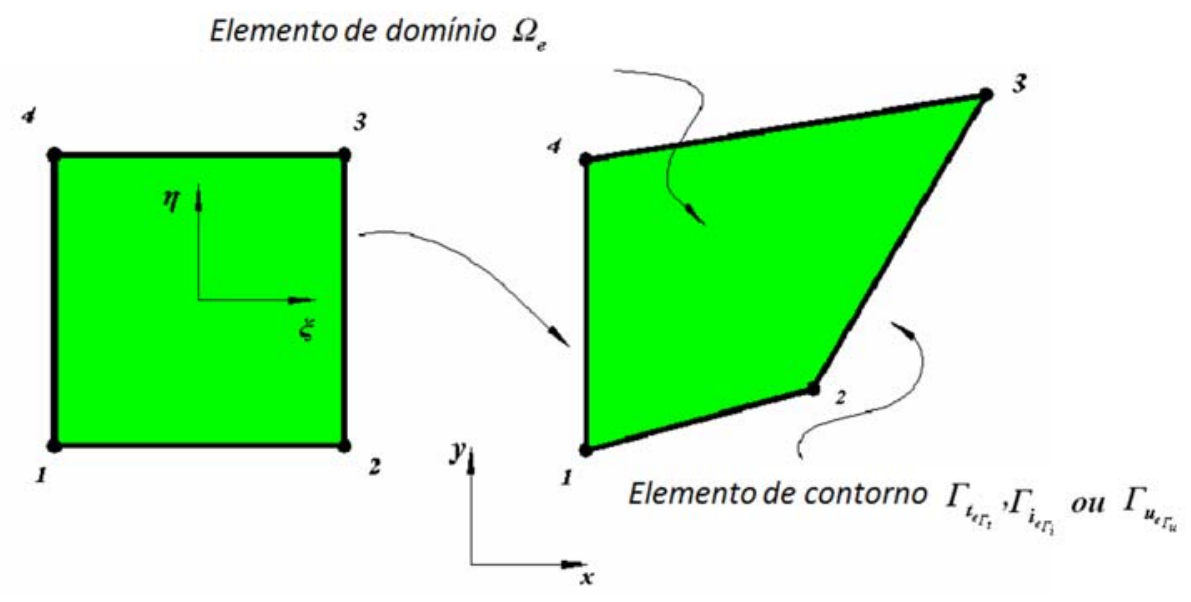

Figura A.1 - Elemento quadrilateral de quatro nós. Fonte: Góis (2009).

São utilizadas as funções Lagrangianas bilineares clássicas para transformação das coordenadas do domínio paramétrico para o domínio físico, sendo representadas em relação a um sistema de referência cartesiano $(\xi \eta)$ localizado no centro do elemento (vide Figura A.1), onde $\xi$ e $\eta$ são coordenadas adimensionais variando entre -01 e 01 .

$\varphi_{1}=\frac{1}{4}(1-\xi)(1-\eta)$

$\varphi_{2}=\frac{1}{4}(1+\xi)(1-\eta)$

$\varphi_{3}=\frac{1}{4}(1+\xi)(1+\eta)$

$\varphi_{4}=\frac{1}{4}(1-\xi)(1+\eta)$

Define-se a matriz $\boldsymbol{M}$ como a composição das funções de interpolação apresentadas da eq.(A.23) a eq.(A.26), e o vetor $\boldsymbol{c}$ que armazena as coordenadas cartesianas nodais do elemento finito.

$$
\boldsymbol{M}=\left[\begin{array}{cccccccc}
\varphi_{1} & 0 & \varphi_{2} & 0 & \varphi_{3} & 0 & \varphi_{4} & 0 \\
0 & \varphi_{1} & 0 & \varphi_{2} & 0 & \varphi_{3} & 0 & \varphi_{4}
\end{array}\right]
$$


$\boldsymbol{c}^{\mathbf{T}}=\left[\begin{array}{llllllll}x_{1} & y_{1} & x_{2} & y_{2} & x_{3} & y_{3} & x_{4} & y_{4}\end{array}\right]$

Assim, as coordenadas $x$ e $y$ globais de um ponto do elemento finito podem ser mapeadas conforme a eq.(A.29).

$\left\{\begin{array}{l}x \\ y\end{array}\right\}=M c$

Com a transformação de coordenadas é possível escrever os operadores diferenciais no referencial paramétrico em função dos definidos no referencial global:

$$
\left\{\begin{array}{c}
\frac{\partial}{\partial \xi} \\
\frac{\partial}{\partial \eta}
\end{array}\right\}=J\left\{\begin{array}{l}
\frac{\partial}{\partial x} \\
\frac{\partial}{\partial y}
\end{array}\right\}
$$

sendo $\boldsymbol{J}$ o jacobiano, expresso pela eq.(A.31).

$\boldsymbol{J}=\left[\begin{array}{ll}\frac{\partial x}{\partial \xi} & \frac{\partial y}{\partial \xi} \\ \frac{\partial x}{\partial \eta} & \frac{\partial y}{\partial \eta}\end{array}\right]$

Considere-se um elemento no contorno $\boldsymbol{\Gamma}\left(\boldsymbol{\Gamma}_{\boldsymbol{u}_{\boldsymbol{e}_{\boldsymbol{u}}}}, \boldsymbol{\Gamma}_{\boldsymbol{t}_{\boldsymbol{e}_{\boldsymbol{t}}}}, \boldsymbol{\Gamma}_{\boldsymbol{i}_{\boldsymbol{e}_{\boldsymbol{i}}}}\right)$ coincidente com um dos lados do elemento quadrilateral $\boldsymbol{\Omega}_{\boldsymbol{e}}$ (Figura A.2). 


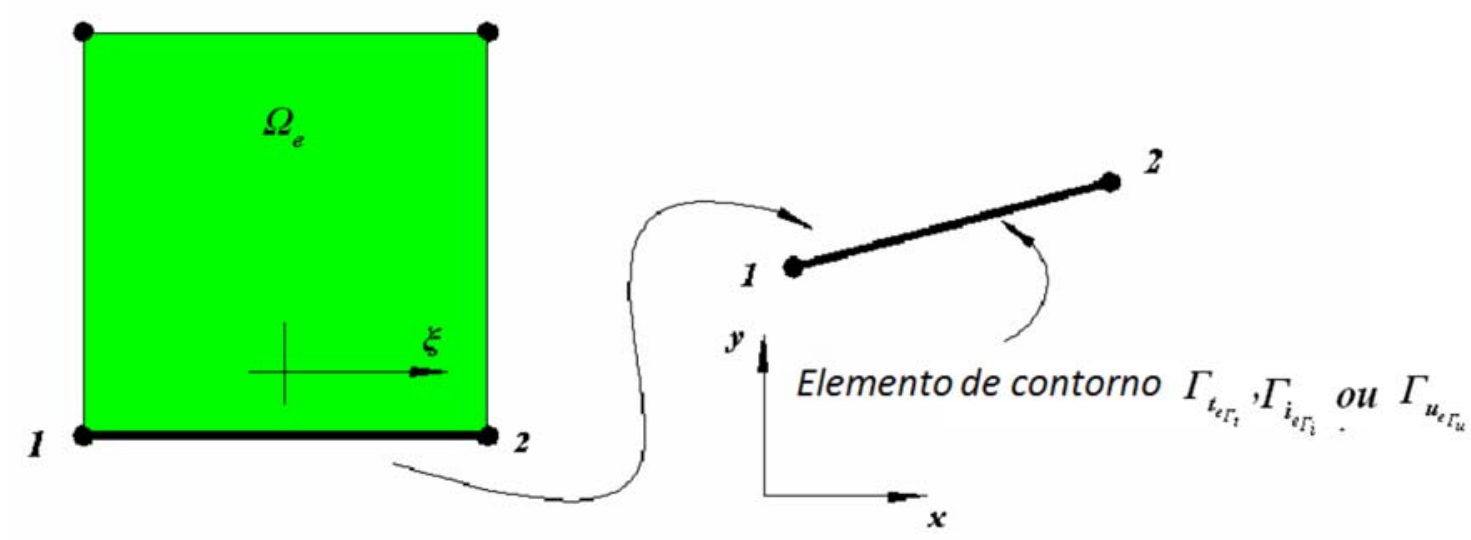

Figura A.2 - Elemento finito linear com sistema local de referência com origem no seu centro. Fonte: Góis (2009).

No mapeamento de coordenadas do elemento linear no contorno paramétrico para o contorno físico utilizam-se as funções Lagrangianas clássicas. Como exemplo, para o elemento em $\eta=-01$ tais funções tem a seguinte apresentação:

$\psi_{1}=-\frac{1}{2}(\xi-1)$

$\psi_{2}=\frac{1}{2}(\xi+1)$

onde $\xi$ é a coordenada adimensional com origem no centro do elemento e variando entre -01 e 01.

De forma análoga é possível obter as equações de $\psi_{1}$ e $\psi_{2}$ para os elementos de contorno que compõem os demais lados do elemento de domínio, desde que se façam as devidas mudanças na definição da coordenada adimensional.

Define-se a matriz $\boldsymbol{M}_{\boldsymbol{\Gamma}}$ composta pelas funções de interpolação $\psi_{1}$ e $\psi_{2}$, dadas pelas eq.(A.32) e eq.(A.33), e o vetor $\boldsymbol{c}_{\boldsymbol{\Gamma}}$ que guarda as coordenadas nodais do elemento finito de contorno.

$\boldsymbol{M}_{\boldsymbol{\Gamma}}=\left[\begin{array}{cccc}\psi_{1} & 0 & \psi_{2} & 0 \\ 0 & \psi_{1} & 0 & \psi_{2}\end{array}\right]$ 
$\boldsymbol{c}_{\Gamma}^{\mathbf{T}}=\left[\begin{array}{llll}x_{1} & y_{1} & x_{2} & y_{2}\end{array}\right]$

Com isso, as coordenadas globais de um ponto da rede de elementos finitos lineares podem ser mapeadas a partir da seguinte transformação:

$\left\{\begin{array}{l}x \\ y\end{array}\right\}=M_{\Gamma} c_{\Gamma}$

De acordo com a convenção isoparamétrica adotada, os elementos de contorno representam uma reta com $\xi$ ou $\eta$ constante. Assim, o cálculo do diferencial de comprimento do elemento de contorno é dado conforme a seguir:

$d s=\sqrt{\left(\frac{\partial x}{\partial \xi}\right)^{2}+\left(\frac{\partial y}{\partial \xi}\right)^{2}} \partial \xi \quad(\eta$ constante $)$

$d s=\sqrt{\left(\frac{\partial x}{\partial \eta}\right)^{2}+\left(\frac{\partial y}{\partial \eta}\right)^{2}} \partial \eta \quad(\xi$ constante $)$

Diante do exposto, para aproximação das variáveis de tensão e deslocamento do domínio serão adotadas as mesmas funções utilizadas para aproximação da geometria do elemento quadrilateral de quatro nós. Assim, tem-se:

$\boldsymbol{S}_{\boldsymbol{\Omega}_{e}}=\left[\begin{array}{cccccccccccc}\varphi_{1} & 0 & 0 & \varphi_{2} & 0 & 0 & \varphi_{3} & 0 & 0 & \varphi_{4} & 0 & 0 \\ 0 & \varphi_{1} & 0 & 0 & \varphi_{2} & 0 & 0 & \varphi_{3} & 0 & 0 & \varphi_{4} & 0 \\ 0 & 0 & \varphi_{1} & 0 & 0 & \varphi_{2} & 0 & 0 & \varphi_{3} & 0 & 0 & \varphi_{4}\end{array}\right]$

$\boldsymbol{U}_{\Omega_{e}}=\left[\begin{array}{cccccccc}\varphi_{1} & 0 & \varphi_{2} & 0 & \varphi_{3} & 0 & \varphi_{4} & 0 \\ 0 & \varphi_{1} & 0 & \varphi_{2} & 0 & \varphi_{3} & 0 & \varphi_{4}\end{array}\right]$

Com isso, os campos incógnitos do domínio são dados por:

$\boldsymbol{s}_{\boldsymbol{\Omega}_{e}}^{\mathbf{T}}=\left[\begin{array}{llllllllllll}\sigma_{x_{1}} & \sigma_{y_{1}} & \tau_{x y_{1}} & \sigma_{x_{2}} & \sigma_{y_{2}} & \tau_{x y_{2}} & \sigma_{x_{3}} & \sigma_{y_{3}} & \tau_{x y_{3}} & \sigma_{x_{4}} & \sigma_{y_{4}} & \tau_{x y_{4}}\end{array}\right]$ 


$$
\boldsymbol{q}_{\boldsymbol{\Omega}_{e}}^{\mathbf{T}}=\left[\begin{array}{llllllll}
u_{x_{1}} & u_{y_{1}} & u_{x_{2}} & u_{y_{2}} & u_{x_{3}} & u_{y_{3}} & u_{x_{4}} & u_{y_{4}}
\end{array}\right]
$$

Analogamente, as funções aproximativas para os deslocamentos no contorno $\boldsymbol{\Gamma}\left(\boldsymbol{\Gamma}_{\boldsymbol{u}_{\boldsymbol{e}_{\boldsymbol{u}}}}, \boldsymbol{\Gamma}_{\boldsymbol{t}_{\boldsymbol{e}_{\boldsymbol{t}}}}, \boldsymbol{\Gamma}_{\boldsymbol{i}_{\boldsymbol{e}_{\boldsymbol{i}}}}\right)$ são as mesmas definidas para a aproximação da geometria do elemento linear de contorno. Logo:

$$
\boldsymbol{U}_{\boldsymbol{\Gamma}_{\boldsymbol{t}_{\boldsymbol{\Gamma}_{\boldsymbol{t}}}}}, \boldsymbol{U}_{\boldsymbol{\Gamma}_{\boldsymbol{i}_{\boldsymbol{e}_{\boldsymbol{i}}}}}=\left[\begin{array}{cccc}
\psi_{1} & 0 & \psi_{2} & 0 \\
0 & \psi_{1} & 0 & \psi_{2}
\end{array}\right]
$$

Com isso, o campo incógnito do contorno (externo ou interno) é dado por:

$$
\boldsymbol{q}_{\boldsymbol{\Gamma}_{\boldsymbol{e}_{\Gamma_{t}}}}^{\mathbf{T}}, \boldsymbol{q}_{\Gamma_{\boldsymbol{\Gamma}_{\Gamma_{i}}}}^{\mathbf{T}}=\left[\begin{array}{llll}
u_{x_{1}} & u_{y_{1}} & u_{x_{2}} & u_{y_{2}}
\end{array}\right]
$$

Vale lembrar que todas as funções aproximativas envolvidas na FHMT são atreladas aos nós. Assim, utilizando a metodologia de enriquecimento nodal do MEFG ampliam-se as bases de aproximação com funções polinomiais. Deste modo, as aproximações para os campos incógnitos da FHMT são dados da seguinte forma:

$\boldsymbol{\sigma}=\sum_{j=1}^{N} S_{\Omega_{j}}\left\{s_{\Omega_{j}}+\sum_{i=1}^{n_{e}} h_{j i} b_{j i}\right\}$

$\boldsymbol{u}=\sum_{j=1}^{N} U_{\Omega_{j}}\left\{u_{\Omega_{j}}+\sum_{i=1}^{n_{e}} h_{j i} c_{j i}\right\}$

$\boldsymbol{u}_{\Gamma}=\sum_{j=1}^{N_{\Gamma}} U_{\Gamma_{j}}\left\{u_{\Gamma_{j}}+\sum_{k=1}^{n_{e_{\Gamma}}} h_{j k} d_{j k}\right\}$

onde $h_{j i}\left(j i=1, \ldots, n_{e}\right)$ e $h_{j k}\left(j k=1, \ldots, n_{e_{\Gamma}}\right)$ são as funções de enriquecimento do domínio e do contorno, respectivamente, sendo $n_{e}$ e $n_{e_{\Gamma}}$ os números total de funções escolhidas para o domínio e o contorno, respectivamente. Sendo, também, $b_{j i}, c_{j i}$ e $d_{j k}$ parâmetros nodais 
adicionais associados às parcelas de enriquecimento em tensão e deslocamento no domínio, e deslocamento no contorno, respectivamente.

Comenta-se, anteriormente, que o enriquecimento das bases aproximativas do MEFG deve ser realizado no domínio global. Para isto, recomenda-se que:

$h_{j i}\left(\xi_{j}, \eta_{j}\right)=0$

$h_{j k}\left(\xi_{j}\right)=0$

onde $\left(\xi_{j}, \eta_{j}\right)$ são as coordenadas adimensionais dos nós $j$ dos elementos de domínio e de contorno. Esta propriedade das eq.(A.48) e eq.(A.49) determina um tipo de funções de enriquecimento denominadas de funções bolhas, sendo este procedimento vantajoso porque preserva o significado físico dos graus de liberdade nodais originais.

Desta forma, as bases aproximativas originais, dadas pelas eq.(A.39), eq.(A.40) e eq.(A.43), tem seus termos não nulos escritos da seguinte forma:

$\boldsymbol{S}_{\Omega_{e}}=\left[\begin{array}{llll}\varphi_{1} \Delta_{1} & \varphi_{2} \Delta_{2} & \varphi_{3} \Delta_{3} & \varphi_{4} \Delta_{4}\end{array}\right]$

$\boldsymbol{U}_{\boldsymbol{\Omega}_{e}}=\left[\begin{array}{llll}\varphi_{1} \Delta_{1} & \varphi_{2} \Delta_{2} & \varphi_{3} \Delta_{3} & \varphi_{4} \Delta_{4}\end{array}\right]$

$\boldsymbol{U}_{\boldsymbol{\Gamma}_{\boldsymbol{t}_{\boldsymbol{\Gamma}_{\boldsymbol{t}}}}}=\boldsymbol{U}_{\boldsymbol{\Gamma}_{\boldsymbol{i}_{\boldsymbol{\Gamma}_{\boldsymbol{i}}}}}=\left[\begin{array}{ll}\psi_{1} \Delta_{\boldsymbol{\Gamma}_{1}} & \psi_{2} \Delta_{\boldsymbol{\Gamma}_{2}}\end{array}\right]$

onde $\Delta_{j}(j=1, \ldots, 4)$ e $\Delta_{\Gamma_{j}}(j=1,2)$ são as matrizes de enriquecimento polinomial do nó de domínio e contorno, respectivamente.

Considera-se que as matrizes de enriquecimento sejam dadas por:

$\Delta_{j}=\left[\begin{array}{llllll}I_{3} & h_{j 1} I_{3} & \ldots & h_{j k} I_{3} & \ldots & h_{j n_{e}} I_{3}\end{array}\right]$

para o campo de tensões no domínio, 
$\Delta_{j}=\left[\begin{array}{llllll}I_{2} & h_{j 1} I_{2} & \ldots & h_{j k} I_{2} & \ldots & h_{j n_{e}} I_{2}\end{array}\right]$

para o campo de deslocamentos no domínio e

$\Delta_{\Gamma_{j}}=\left[\begin{array}{llllll}I_{2} & h_{j 1} I_{2} & \ldots & h_{j k} I_{2} & \ldots & h_{j n_{e_{\Gamma}}} I_{2}\end{array}\right]$

para o campo de deslocamentos no contorno.

Nas eq.(A.53) a eq.(A.55) $\boldsymbol{I}_{2}$ e $\boldsymbol{I}_{3}$ são matrizes identidades de segunda e terceira ordem, respectivamente. Nestas equações tal forma é adotada para que se preserve a estrutura convencional da formulação em estudo, quando as funções de enriquecimento forem nulas.

Assim, os monômios $x$ e $y$ são representados por:

$h_{j n_{e}}(\xi, \eta)=\frac{\left(\varphi_{1} x_{1}+\varphi_{2} x_{2}+\varphi_{3} x_{3}+\varphi_{4} x_{4}\right)-x_{j}}{h_{j}}$

$h_{j n_{e}}(\xi, \eta)=\frac{\left(\varphi_{1} y_{1}+\varphi_{2} y_{2}+\varphi_{3} y_{3}+\varphi_{4} y_{4}\right)-y_{j}}{h_{j}}$

para os campos de domínio e

$h_{j n_{e_{\Gamma}}}(\xi)=\frac{\left(\psi_{1} x_{1}+\psi_{2} x_{2}\right)-x_{j}}{h_{j}}$

$h_{j n_{e_{\Gamma}}}(\xi)=\frac{\left(\psi_{1} y_{1}+\psi_{2} y_{2}\right)-y_{j}}{h_{j}}$

para os campos de contorno, onde $h_{j}$ é o raio da nuvem de um nó $j$ qualquer. Com esta convenção, é possível enriquecer os campos de domínio e contorno com as bases $L_{5(\alpha)}^{\Omega}=$ $\left\{1, x, y, x y, x^{2}, y^{2}\right\}$ e $L_{2(\alpha)}^{\Gamma}=\{1, x, y\}$, respectivamente.

Assim, para qualquer nó $j \therefore(j=1, \ldots, 4)$ do elemento finito retangular $e$, escolhido para ser enriquecido, pode-se representar: 
$\boldsymbol{S}_{\Omega_{\mathrm{e}}}^{\mathrm{T}} \boldsymbol{f} \boldsymbol{S}_{\Omega_{\mathrm{e}}}=\left[\varphi_{\alpha} \varphi_{\beta} \Delta_{\alpha}^{\mathrm{T}} \boldsymbol{f} \Delta_{\boldsymbol{\beta}}\right] ; \alpha, \beta=1, \ldots, 4$

$\boldsymbol{F}_{\boldsymbol{e}}=\left[\boldsymbol{F}_{\alpha \boldsymbol{\beta}}\right] \therefore \boldsymbol{F}_{\alpha \boldsymbol{\beta}}=\left(\int_{\boldsymbol{\Omega}_{\mathrm{e}}} \varphi_{\alpha} \varphi_{\beta} \Delta_{\alpha}^{\mathrm{T}} \mathrm{f} \Delta_{\boldsymbol{\beta}} \boldsymbol{d} \boldsymbol{\Omega}\right)$

$\therefore \Delta_{\alpha}^{\mathrm{T}} f \Delta_{\beta}=\left[\begin{array}{ccccc}f & \cdots & h_{j \beta} f & \cdots & h_{n_{e} \beta} f \\ \vdots & \ddots & \vdots & \ddots & \vdots \\ h_{j \alpha} f & \cdots & h_{j \alpha} h_{j \beta} f & \cdots & h_{j \alpha} h_{n_{e} \beta} f \\ \vdots & \ddots & \vdots & \ddots & \vdots \\ h_{n_{e} \alpha} f & \cdots & h_{n_{e} \alpha} h_{j \beta} f & \cdots & h_{n_{e} \alpha} h_{n_{e} \beta} f\end{array}\right]$

$\boldsymbol{L} \boldsymbol{S}_{\boldsymbol{\Omega}_{e}}=\boldsymbol{L}\left[\begin{array}{llll}\varphi_{1} \Delta_{1} & \varphi_{2} \Delta_{2} & \varphi_{3} \Delta_{3} & \varphi_{4} \Delta_{4}\end{array}\right]=$

$$
=\left[\begin{array}{lll}
\left(\boldsymbol{L} \varphi_{1}\right) \Delta_{1}+\varphi_{1}\left(\boldsymbol{L} \Delta_{1}\right) & \cdots & \left(\boldsymbol{L} \varphi_{4}\right) \Delta_{4}+\varphi_{4}\left(\boldsymbol{L} \Delta_{4}\right)
\end{array}\right]
$$

$\therefore\left(\boldsymbol{L} \boldsymbol{S}_{\boldsymbol{\Omega}_{e}}\right)^{\mathrm{T}}=\left[\begin{array}{c}\Delta_{\mathbf{1}}^{\mathrm{T}}\left(\boldsymbol{L} \varphi_{1}\right)^{\mathrm{T}}+\varphi_{1}\left(\boldsymbol{L} \Delta_{1}\right)^{\mathrm{T}} \\ \vdots \\ \Delta_{4}^{\mathrm{T}}\left(\boldsymbol{L} \varphi_{4}\right)^{\mathrm{T}}+\varphi_{4}\left(\boldsymbol{L} \Delta_{4}\right)^{\mathrm{T}}\end{array}\right]$

$\left(\boldsymbol{L} \boldsymbol{S}_{\boldsymbol{\Omega}_{e}}\right)^{\mathrm{T}} \boldsymbol{U}_{\boldsymbol{\Omega}_{e}}=\left[\Delta_{\boldsymbol{\alpha}}^{\mathrm{T}}\left(\boldsymbol{L} \varphi_{\alpha}\right)^{\mathrm{T}}+\varphi_{\alpha}\left(\boldsymbol{L} \Delta_{\alpha}\right)^{\mathrm{T}}\right]\left[\varphi_{\beta} \Delta_{\boldsymbol{\beta}}\right] ; \alpha, \beta=1, \ldots, 4$

$\boldsymbol{A}_{\boldsymbol{\Omega}_{e}}=\left[\boldsymbol{A}_{\boldsymbol{\Omega}_{\alpha \beta}}\right] \therefore \boldsymbol{A}_{\boldsymbol{\Omega}_{\alpha \beta}}=\int_{\boldsymbol{\Omega}_{e}}\left[\Delta_{\alpha}^{\mathrm{T}}\left(\boldsymbol{L} \varphi_{\alpha}\right)^{\mathrm{T}}+\varphi_{\alpha}\left(\boldsymbol{L} \Delta_{\alpha}\right)^{\mathrm{T}}\right]\left[\varphi_{\beta} \Delta_{\beta}\right] d \boldsymbol{\Omega}$

$\boldsymbol{U}_{\boldsymbol{\Omega}_{e}}^{\mathrm{T}}=\left[\begin{array}{c}\varphi_{1} \Delta_{\mathbf{1}}^{\mathrm{T}} \\ \vdots \\ \varphi_{4} \Delta_{4}^{\mathrm{T}}\end{array}\right] \therefore \boldsymbol{Q}_{\boldsymbol{\Omega}_{e}}=\left[\boldsymbol{Q}_{\boldsymbol{\Omega}_{\alpha}}\right] \therefore \boldsymbol{Q}_{\Omega_{\alpha}}=\int_{\Omega_{e}} \varphi_{\alpha} \Delta_{\alpha}^{\mathrm{T}} \bar{b} \boldsymbol{d} \boldsymbol{\Omega} ; \alpha=1, \ldots, 4$

Analogamente, para qualquer nó $j \therefore(j=1,2)$ do elemento finito linear pertencente contorno $\boldsymbol{\Gamma}\left(\boldsymbol{\Gamma}_{\boldsymbol{u}_{\boldsymbol{e}_{\boldsymbol{F}_{u}}}}, \boldsymbol{\Gamma}_{\boldsymbol{t}_{\boldsymbol{e}_{\boldsymbol{t}}}}, \boldsymbol{\Gamma}_{\boldsymbol{i}_{\boldsymbol{e}_{i}}}\right)$ de um elemento quadrilateral, escolhido para ser enriquecido, pode-se representar: 


$$
\begin{aligned}
& \boldsymbol{N} \boldsymbol{S}_{\boldsymbol{\Omega}_{\boldsymbol{e}}}=\left[\begin{array}{llll}
\left(\boldsymbol{N} \varphi_{1}\right) \Delta_{1} & \left(\boldsymbol{N} \varphi_{2}\right) \Delta_{2} & \left(\boldsymbol{N} \varphi_{3}\right) \Delta_{3} & \left(\boldsymbol{N} \varphi_{4}\right) \Delta_{4}
\end{array}\right] \\
& \therefore\left(N S_{\Omega_{e}}\right)^{\mathrm{T}}=\left[\begin{array}{c}
\Delta_{1}^{\mathrm{T}}\left(N \varphi_{1}\right)^{\mathrm{T}} \\
\vdots \\
\Delta_{4}^{\mathrm{T}}\left(\boldsymbol{N} \varphi_{4}\right)^{\mathrm{T}}
\end{array}\right] \\
& \left(\boldsymbol{N} \boldsymbol{S}_{\boldsymbol{\Omega}_{e}}\right)^{\mathbf{T}} \boldsymbol{U}_{\boldsymbol{\Gamma}_{t_{\boldsymbol{e}_{\boldsymbol{t}}}}}=\left(\boldsymbol{N} \boldsymbol{S}_{\boldsymbol{\Omega}_{e}}\right)^{\mathbf{T}} \boldsymbol{U}_{\boldsymbol{\Gamma}_{\boldsymbol{e}_{\boldsymbol{\Gamma}_{\boldsymbol{i}}}}}=\left[\Delta_{\alpha}^{\mathrm{T}}\left(\boldsymbol{N} \varphi_{\alpha}\right)^{\mathbf{T}}\right]\left[\varphi_{\gamma} \Delta_{\boldsymbol{\Gamma}_{\gamma}}\right] ; \begin{array}{c}
\alpha=1, \ldots, 4 \\
\gamma=1,2
\end{array}
\end{aligned}
$$

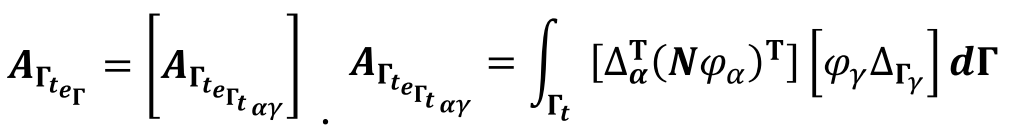

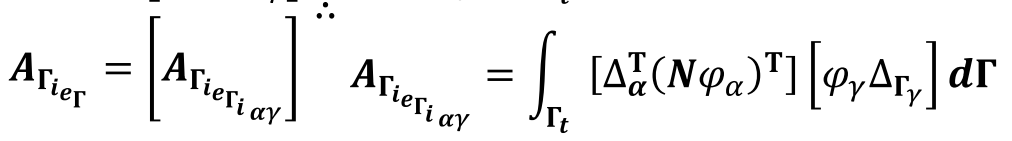

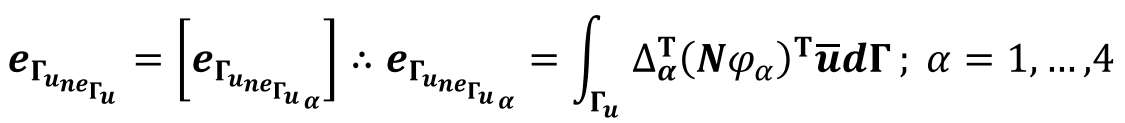

$$
\begin{aligned}
& \boldsymbol{U}_{\boldsymbol{\Gamma}_{t}}^{\mathbf{T}}=\left[\begin{array}{c}
\varphi_{1} \Delta_{\boldsymbol{\Gamma}_{1}}^{\mathbf{T}} \\
\varphi_{2} \Delta_{\boldsymbol{\Gamma}_{2}}^{\mathbf{T}}
\end{array}\right] \therefore \boldsymbol{Q}_{\boldsymbol{\Gamma}_{t_{e_{\Gamma}}}}=\left[\boldsymbol{Q}_{\boldsymbol{\Gamma}_{\boldsymbol{t}_{\boldsymbol{e}_{\gamma}}}}\right] \therefore \boldsymbol{Q}_{\boldsymbol{\Gamma}_{t_{\boldsymbol{}_{\boldsymbol{\Gamma}} \gamma}}}=\int_{\boldsymbol{\Gamma}_{t}} \varphi_{\gamma} \Delta_{\boldsymbol{\Gamma}_{\gamma}}^{\mathbf{T}} \overline{\boldsymbol{t}} \boldsymbol{d} \boldsymbol{\Gamma} ; \gamma=1,2
\end{aligned}
$$

\section{A.2 Modelo discreto da FM(u- $\sigma)$ com enriquecimento nodal}

Este item apresenta o desenvolvimento do modelo discreto para a $\mathrm{FM}(\mathrm{u}-\sigma)$ realizado neste trabalho. Ressalta-se que se tomou como base o item anterior. Vale lembrar que, assim como na FHMT, a FM(u- $\sigma)$ tem suas aproximações atreladas aos nós, podendo ser utilizada a metodologia clássica de enriquecimento do Método das Nuvens $h p$ para os campos de deslocamento e tensão, ambos no domínio.

Considere-se um domínio $\boldsymbol{\Omega}$ composto por $\boldsymbol{n}_{\boldsymbol{\Omega}}$ elementos finitos de domínio, onde $\boldsymbol{\Omega}_{\boldsymbol{e}}\left(\boldsymbol{e}=\mathbf{1}, \ldots, \boldsymbol{n}_{\boldsymbol{\Omega}}\right)$ são os domínios de cada um dos $\boldsymbol{n}_{\boldsymbol{\Omega}}$ elementos. Os vetores $\boldsymbol{S}_{\boldsymbol{\Omega}_{\boldsymbol{e}}}$ e $\boldsymbol{q}_{\boldsymbol{\Omega}_{\boldsymbol{e}}}$ armazenam os parâmetros generalizados correspondentes às aproximações locais para as tensões e deslocamentos.

Os parâmetros locais e globais se relacionam através de matrizes Booleanas, da seguinte forma:

$$
\boldsymbol{s}_{\Omega_{e}}=\left\langle B\left(F_{e}\right)\right\rangle s_{\Omega}
$$


$\boldsymbol{q}_{\Omega_{e}}=\left\langle B\left(\boldsymbol{A}_{e}\right)\right\rangle \boldsymbol{q}_{\Omega}$

As matrizes $\left\langle\boldsymbol{B}\left(\boldsymbol{F}_{\boldsymbol{e}}\right)\right\rangle$ e $\left\langle\boldsymbol{B}\left(\boldsymbol{A}_{\boldsymbol{e}}\right)\right\rangle$ extraem os parâmetros generalizados dos elementos $\boldsymbol{\Omega}_{e}\left(e=1, \ldots, n_{\Omega}\right)$.

Sejam as aproximações locais dos campos de tensão e deslocamento:

$\widehat{\boldsymbol{\sigma}}_{e}=S_{\Omega_{e}} S_{\Omega_{e}}$

$\widehat{\boldsymbol{u}}_{\boldsymbol{e}}=\boldsymbol{U}_{\Omega_{e}} \boldsymbol{q}_{\Omega_{e}}$

Sendo $\boldsymbol{S}_{\boldsymbol{\Omega}_{\boldsymbol{e}}}$ e $\boldsymbol{U}_{\boldsymbol{\Omega}_{\boldsymbol{e}}}$ as matrizes que coletam as funções de interpolação dos campos de tensão e deslocamento. Com a contribuição de cada elemento finito montam-se as matrizes que compõem o sistema resolvente da $\operatorname{FM}(u-\sigma)$, dado pela eq.(3.73).

$F=\sum_{e=1}^{n_{\Omega}}\left\langle B\left(F_{e}\right)\right\rangle^{\mathrm{T}} F_{e}\left\langle B\left(F_{e}\right)\right\rangle$

$A=\sum_{e=1}^{n_{\Omega}}\left\langle B\left(F_{e}\right)\right\rangle^{\mathrm{T}} \boldsymbol{A}_{e}\left\langle B\left(\boldsymbol{A}_{e}\right)\right\rangle$

$Q=\sum_{e=1}^{n_{\Omega}}\left\langle B\left(A_{e}\right)\right\rangle^{\mathrm{T}} Q_{e}$

onde:

$F_{e}=\int_{\Omega} S_{\Omega_{\mathrm{e}}}^{\mathrm{T}} f S_{\Omega_{\mathrm{e}}} d \Omega$

$A_{e}=\int_{\Omega}\left(L S_{\Omega_{e}}\right)^{\mathrm{T}} U_{\Omega_{e}} d \Omega$ 
$Q_{e}=\int_{\Omega} U_{\Omega_{\mathrm{e}}}^{\mathrm{T}} \bar{b} d \Omega+\int_{\Gamma_{t}} U_{\Omega_{\mathrm{e}}}^{\mathrm{T}} \bar{t} d \Gamma$

Com isso, as matrizes que guardam as aproximações dos campos de tensões e deslocamentos são constituídas por submatrizes cujas dimensões estão associadas ao número de nós dos elementos, ao número de graus de liberdade definido em cada nó, ao número de nós enriquecidos e aos graus de enriquecimentos nodais adotados.

A Figura A.3 apresenta o elemento finito misto de dois campos quadrilateral de quatro nós, sendo também exemplificada sua convenção de numeração nodal (sentido anti-horário).

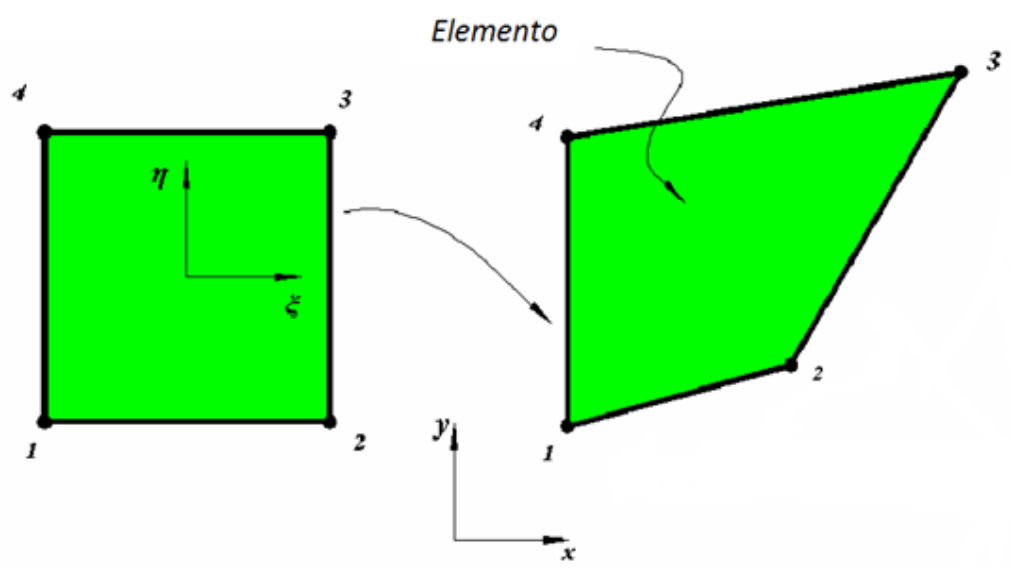

Figura A.3 - Elemento quadrilateral de quatro nós.

São utilizadas as funções Lagrangianas bilineares clássicas para transformação das coordenadas do domínio paramétrico para o domínio físico, sendo representadas em relação a um sistema de referência cartesiano $(\xi \eta)$ localizado no centro do elemento (vide Figura A.3), onde $\xi$ e $\eta$ são coordenadas adimensionais variando entre -01 e 01 .

$\varphi_{1}=\frac{1}{4}(1-\xi)(1-\eta)$

$\varphi_{2}=\frac{1}{4}(1+\xi)(1-\eta)$

$\varphi_{3}=\frac{1}{4}(1+\xi)(1+\eta)$ 
$\varphi_{4}=\frac{1}{4}(1-\xi)(1+\eta)$

Define-se a matriz $\boldsymbol{M}$ como a composição das funções de interpolação apresentadas da eq.(A.76) a eq.(A.79), e o vetor $\boldsymbol{c}$ que armazena as coordenadas cartesianas nodais do elemento finito.

$\boldsymbol{M}=\left[\begin{array}{cccccccc}\varphi_{1} & 0 & \varphi_{2} & 0 & \varphi_{3} & 0 & \varphi_{4} & 0 \\ 0 & \varphi_{1} & 0 & \varphi_{2} & 0 & \varphi_{3} & 0 & \varphi_{4}\end{array}\right]$

$\boldsymbol{c}^{\mathbf{T}}=\left[\begin{array}{llllllll}x_{1} & y_{1} & x_{2} & y_{2} & x_{3} & y_{3} & x_{4} & y_{4}\end{array}\right]$

Assim, as coordenadas $x$ e $y$ globais de um ponto do elemento finito podem ser mapeadas conforme a eq.(A.82).

$\left\{\begin{array}{l}x \\ y\end{array}\right\}=M c$

Com a transformação de coordenadas é possível escrever os operadores diferenciais no referencial paramétrico em função dos definidos no referencial global:

$\left\{\begin{array}{c}\frac{\partial}{\partial \xi} \\ \frac{\partial}{\partial \eta}\end{array}\right\}=J\left\{\begin{array}{l}\frac{\partial}{\partial x} \\ \frac{\partial}{\partial y}\end{array}\right\}$

sendo $\boldsymbol{J}$ o jacobiano, expresso pela eq.(A.31)(A.84).

$J=\left[\begin{array}{ll}\frac{\partial x}{\partial \xi} & \frac{\partial y}{\partial \xi} \\ \frac{\partial x}{\partial \eta} & \frac{\partial y}{\partial \eta}\end{array}\right]$ 
Apêndice A

Diante do exposto, para aproximação das variáveis de tensão e deslocamento são adotadas as mesmas funções utilizadas para aproximação da geometria do elemento quadrilateral de quatro nós. Assim, tem-se:

$$
\begin{aligned}
\boldsymbol{S}_{\Omega_{e}} & =\left[\begin{array}{cccccccccccc}
\varphi_{1} & 0 & 0 & \varphi_{2} & 0 & 0 & \varphi_{3} & 0 & 0 & \varphi_{4} & 0 & 0 \\
0 & \varphi_{1} & 0 & 0 & \varphi_{2} & 0 & 0 & \varphi_{3} & 0 & 0 & \varphi_{4} & 0 \\
0 & 0 & \varphi_{1} & 0 & 0 & \varphi_{2} & 0 & 0 & \varphi_{3} & 0 & 0 & \varphi_{4}
\end{array}\right] \\
\boldsymbol{U}_{\Omega_{e}} & =\left[\begin{array}{cccccccc}
\varphi_{1} & 0 & \varphi_{2} & 0 & \varphi_{3} & 0 & \varphi_{4} & 0 \\
0 & \varphi_{1} & 0 & \varphi_{2} & 0 & \varphi_{3} & 0 & \varphi_{4}
\end{array}\right]
\end{aligned}
$$

Com isso, os campos incógnitos do domínio são dados por:

$$
\begin{aligned}
& \boldsymbol{s}_{\boldsymbol{\Omega}_{\boldsymbol{e}}}^{\mathbf{T}}=\left[\begin{array}{llllllllllll}
\sigma_{x_{1}} & \sigma_{y_{1}} & \tau_{x y_{1}} & \sigma_{x_{2}} & \sigma_{y_{2}} & \tau_{x y_{2}} & \sigma_{x_{3}} & \sigma_{y_{3}} & \tau_{x y_{3}} & \sigma_{x_{4}} & \sigma_{y_{4}} & \tau_{x y_{4}}
\end{array}\right] \\
& \boldsymbol{q}_{\boldsymbol{\Omega}_{\boldsymbol{e}}}^{\mathbf{T}}=\left[\begin{array}{lllllllll}
u_{x_{1}} & u_{y_{1}} & u_{x_{2}} & u_{y_{2}} & u_{x_{3}} & u_{y_{3}} & u_{x_{4}} & u_{y_{4}}
\end{array}\right]
\end{aligned}
$$

Assim, utilizando a metodologia de enriquecimento nodal do MEFG, neste trabalho, ampliam-se as bases de aproximação com funções polinomiais. Deste modo, as aproximações para os campos incógnitos da $\mathrm{FM}(\mathrm{u}-\sigma)$ são dados da seguinte forma:

$$
\begin{aligned}
& \boldsymbol{\sigma}=\sum_{j=1}^{N} S_{\Omega_{j}}\left\{\sigma_{j}+\sum_{i=1}^{n_{e}} h_{j i} b_{j i}\right\} \\
& \boldsymbol{u}=\sum_{j=1}^{N} U_{\Omega_{j}}\left\{u_{j}+\sum_{i=1}^{n_{e}} h_{j i} c_{j i}\right\}
\end{aligned}
$$

onde $h_{j i}\left(j i=1, \ldots, n_{e}\right)$ são as funções enriquecedoras, sendo $n_{e}$ o número de funções de enriquecimento escolhidas. Sendo, também, $b_{j i}$ e $c_{j i}$ parâmetros nodais adicionais associados às parcelas de enriquecimento em tensão e deslocamento, respectivamente.

Comenta-se, anteriormente, que o enriquecimento das bases aproximativas do MEFG deve ser realizado no domínio global. Para isto, recomenda-se que: 
$h_{j i}\left(\xi_{j}, \eta_{j}\right)=0$

onde $\left(\xi_{j}, \eta_{j}\right)$ são as coordenadas adimensionais dos nós $j$ dos elementos. Esta propriedade da eq.(A.91) determina um tipo de função de enriquecimento denominada de função bolha, sendo este procedimento vantajoso porque preserva o significado físico de graus de liberdade nodais originais, conforme citado anteriormente.

Desta forma, as bases aproximativas originais, dadas pelas eq.(A.85) e eq.(A.86) tem seus termos não nulos escritos da seguinte forma:

$\boldsymbol{S}_{\Omega_{e}}=\left[\begin{array}{llll}\varphi_{1} \Delta_{1} & \varphi_{2} \Delta_{2} & \varphi_{3} \Delta_{3} & \varphi_{4} \Delta_{4}\end{array}\right]$

$\boldsymbol{U}_{\boldsymbol{\Omega}_{e}}=\left[\begin{array}{llll}\varphi_{1} \Delta_{1} & \varphi_{2} \Delta_{2} & \varphi_{3} \Delta_{3} & \varphi_{4} \Delta_{4}\end{array}\right]$

onde $\Delta_{j}(j=1, \ldots, 4)$ são as matrizes de enriquecimento polinomial do nó.

Considere agora que as matrizes de enriquecimento sejam dadas por:

$\Delta_{j}=\left[\begin{array}{llllll}I_{3} & h_{j 1} I_{3} & \ldots & h_{j k} I_{3} & \ldots & h_{j n_{e}} I_{3}\end{array}\right]$

para o campo de tensões e

$\Delta_{j}=\left[\begin{array}{llllll}I_{2} & h_{j 1} I_{2} & \ldots & h_{j k} I_{2} & \ldots & h_{j n_{e}} I_{2}\end{array}\right]$

para o campo de deslocamentos.

Nas eq.(A.94) e eq.(A.95) $\boldsymbol{I}_{2}$ e $\boldsymbol{I}_{3}$ são matrizes identidades de segunda e terceira ordem, respectivamente. Nestas equações tal forma é adotada para que se preserve a estrutura convencional da formulação em estudo, quando as funções de enriquecimento forem nulas. Assim, as formas adotadas para as funções bolhas para $x$ e $y$ são, respectivamente: 
Apêndice A

$h_{j n_{e}}(\xi, \eta)=\frac{\left(\varphi_{1} x_{1}+\varphi_{2} x_{2}+\varphi_{3} x_{3}+\varphi_{4} x_{4}\right)-x_{j}}{h_{j}}$

$h_{j n_{e}}(\xi, \eta)=\frac{\left(\varphi_{1} y_{1}+\varphi_{2} y_{2}+\varphi_{3} y_{3}+\varphi_{4} y_{4}\right)-y_{j}}{h_{j}}$

sendo os demais monômios da base $L_{5(\alpha)}=\left\{1, x, y, x y, x^{2}, y^{2}\right\}$ obtidos com estas equações.

Assim, para qualquer nó $j \therefore(j=1, \ldots, 4)$ do elemento finito retangular $e$, escolhido para ser enriquecido, podem-se representar:

$$
\begin{aligned}
& \boldsymbol{S}_{\Omega_{\mathrm{e}}}^{\mathrm{T}} \boldsymbol{f} \boldsymbol{S}_{\boldsymbol{\Omega}_{\mathrm{e}}}=\left[\varphi_{\alpha} \varphi_{\beta} \Delta_{\alpha}^{\mathrm{T}} \boldsymbol{f} \Delta_{\boldsymbol{\beta}}\right] ; \alpha, \beta=1, \ldots, 4 \\
& \boldsymbol{F}_{e}=\left[\boldsymbol{F}_{\alpha \beta}\right] \therefore \boldsymbol{F}_{\alpha \beta}=\left(\int_{\Omega_{\mathrm{e}}} \varphi_{\alpha} \varphi_{\beta} \Delta_{\alpha}^{\mathrm{T}} f \Delta_{\beta} d \boldsymbol{\Omega}\right) \\
& \therefore \Delta_{\alpha}^{\mathrm{T}} \boldsymbol{f} \Delta_{\beta}=\left[\begin{array}{ccccc}
\boldsymbol{f} & \cdots & \boldsymbol{h}_{j \beta} \boldsymbol{f} & \cdots & \boldsymbol{h}_{\boldsymbol{n}_{e} \beta} \boldsymbol{f} \\
\vdots & \ddots & \vdots & \ddots & \vdots \\
\boldsymbol{h}_{j \alpha} \boldsymbol{f} & \cdots & \boldsymbol{h}_{j \alpha} \boldsymbol{h}_{j \beta} \boldsymbol{f} & \cdots & \boldsymbol{h}_{j \alpha} \boldsymbol{h}_{\boldsymbol{n}_{e} \boldsymbol{f}} \boldsymbol{f} \\
\vdots & \ddots & \vdots & \ddots & \vdots \\
\boldsymbol{h}_{n_{e} \alpha} \boldsymbol{f} & \cdots & \boldsymbol{h}_{n_{e} \alpha} \boldsymbol{h}_{j \beta} \boldsymbol{f} & \cdots & \boldsymbol{h}_{n_{e} \alpha} \boldsymbol{h}_{n_{e} \beta} \boldsymbol{f}
\end{array}\right]
\end{aligned}
$$




$$
\begin{aligned}
& \boldsymbol{L} \boldsymbol{S}_{\boldsymbol{\Omega}_{e}}=\boldsymbol{L}\left[\varphi_{1} \Delta_{1} \quad \varphi_{2} \Delta_{2} \quad \varphi_{3} \Delta_{3} \quad \varphi_{4} \Delta_{4}\right]= \\
& =\left[\begin{array}{lll}
\left(\boldsymbol{L} \varphi_{1}\right) \Delta_{\mathbf{1}}+\varphi_{1}\left(\boldsymbol{L} \Delta_{\mathbf{1}}\right) & \cdots & \left(\boldsymbol{L} \varphi_{4}\right) \Delta_{\mathbf{4}}+\varphi_{4}\left(\boldsymbol{L} \Delta_{4}\right)
\end{array}\right] \\
& \therefore\left(\boldsymbol{L} \boldsymbol{S}_{\Omega_{e}}\right)^{\mathrm{T}}=\left[\begin{array}{c}
\Delta_{1}^{\mathrm{T}}\left(\boldsymbol{L} \varphi_{1}\right)^{\mathrm{T}}+\varphi_{1}\left(\boldsymbol{L} \Delta_{1}\right)^{\mathrm{T}} \\
\vdots \\
\Delta_{4}^{\mathrm{T}}\left(\boldsymbol{L} \varphi_{4}\right)^{\mathrm{T}}+\varphi_{4}\left(\boldsymbol{L} \Delta_{4}\right)^{\mathrm{T}}
\end{array}\right] \\
& \left(\boldsymbol{L} \boldsymbol{S}_{\boldsymbol{\Omega}_{e}}\right)^{\mathbf{T}} \boldsymbol{U}_{\boldsymbol{\Omega}_{e}}=\left[\Delta_{\boldsymbol{\alpha}}^{\mathrm{T}}\left(\boldsymbol{L} \varphi_{\alpha}\right)^{\mathrm{T}}+\varphi_{\alpha}\left(\boldsymbol{L} \Delta_{\boldsymbol{\alpha}}\right)^{\mathrm{T}}\right]\left[\varphi_{\beta} \Delta_{\boldsymbol{\beta}}\right] ; \alpha, \beta=1, \ldots, 4 \\
& \boldsymbol{N} \boldsymbol{S}_{\boldsymbol{\Omega}_{e}}=\left[\begin{array}{llll}
\left(\boldsymbol{N} \varphi_{1}\right) \Delta_{1} & \left(\boldsymbol{N} \varphi_{2}\right) \Delta_{2} & \left(\boldsymbol{N} \varphi_{3}\right) \Delta_{3} & \left(\boldsymbol{N} \varphi_{4}\right) \Delta_{4}
\end{array}\right] \\
& \therefore\left(N S_{\Omega_{e}}\right)^{\mathrm{T}}=\left[\begin{array}{c}
\Delta_{1}^{\mathrm{T}}\left(\boldsymbol{N} \varphi_{1}\right)^{\mathrm{T}} \\
\vdots \\
\Delta_{4}^{\mathrm{T}}\left(\boldsymbol{N} \varphi_{4}\right)^{\mathrm{T}}
\end{array}\right] \\
& \left(\boldsymbol{N} \boldsymbol{S}_{\boldsymbol{\Omega}_{e}}\right)^{\mathrm{T}} \boldsymbol{U}_{\boldsymbol{\Omega}_{e}}=\left(\boldsymbol{N} \boldsymbol{S}_{\boldsymbol{\Omega}_{e}}\right)^{\mathrm{T}} \boldsymbol{U}_{\boldsymbol{\Omega}_{e}}=\left[\Delta_{\alpha}^{\mathrm{T}}\left(\boldsymbol{N} \varphi_{\alpha}\right)^{\mathrm{T}}\right]\left[\varphi_{\alpha} \Delta_{\alpha}\right] ; \alpha=1, \ldots, 4 \\
& \boldsymbol{A}_{e}=\left[\boldsymbol{A}_{\boldsymbol{e}_{\alpha \beta}}\right] \therefore \boldsymbol{A}_{\boldsymbol{e}_{\alpha \beta}} \\
& =\int_{\boldsymbol{\Omega}_{e}}\left[\Delta_{\boldsymbol{\alpha}}^{\mathrm{T}}\left(\boldsymbol{L} \varphi_{\alpha}\right)^{\mathrm{T}}+\varphi_{\alpha}\left(\boldsymbol{L} \Delta_{\alpha}\right)^{\mathrm{T}}\right]\left[\varphi_{\beta} \Delta_{\boldsymbol{\beta}}\right] d \boldsymbol{\Omega} \\
& -\int_{\Gamma_{t}}\left[\Delta_{\alpha}^{\mathrm{T}}\left(\boldsymbol{N} \varphi_{\alpha}\right)^{\mathrm{T}}\right]\left[\varphi_{\beta} \Delta_{\beta}\right] d \Gamma
\end{aligned}
$$

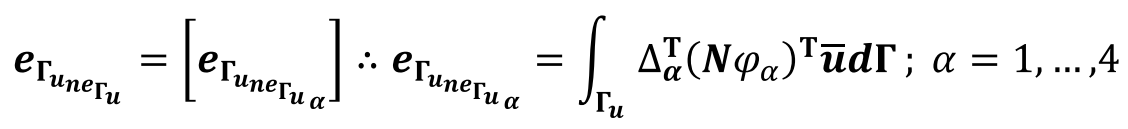

$$
\begin{aligned}
& \boldsymbol{U}_{\boldsymbol{\Omega}_{e}}^{\mathbf{T}}=\left[\begin{array}{c}
\varphi_{1} \Delta_{1}^{\mathbf{T}} \\
\vdots \\
\varphi_{4} \Delta_{4}^{\mathbf{T}}
\end{array}\right] \therefore \boldsymbol{Q}_{e}=\left[\boldsymbol{Q}_{\boldsymbol{e}_{\alpha}}\right] \\
& \therefore \boldsymbol{Q}_{e_{\alpha}}=\int_{\Omega_{e}} \varphi_{\alpha} \Delta_{\alpha}^{\mathrm{T}} \bar{b} d \Omega+\int_{\Gamma_{t}} \varphi_{\alpha} \Delta_{\alpha}^{\mathrm{T}} \overline{\boldsymbol{t}} d \Gamma ; \alpha=1, \ldots, 4
\end{aligned}
$$




\section{APÊNDICE B - Solução de sistemas: método de perturbação}

$\mathrm{Na}$ formulação convencional do $\mathrm{MEFG}$, na aplicação de determinados tipos de enriquecimento, como o polinomial, existe uma grande probabilidade de as funções de forma geradas resultarem linearmente dependentes, conforme exposto por Proença (2010). Tal dependência linear causa singularidade na matriz de rigidez do problema.

Existem vários tipos de abordagens possíveis para resolver este tipo de sistema. Assim, foi proposto Strouboulis et al. (2000) um método de perturbação da diagonal principal. Neste trabalho, o tal método é modificado para resolver os sistemas das formulações não convencionais apresentadas.

Com isso, considerem-se os sistemas das formulações FHMT e FM(u- $\sigma)$, respectivamente, reproduzidos a seguir:

$$
\begin{aligned}
& {\left[\begin{array}{ccc}
\boldsymbol{F} & \boldsymbol{A}_{\boldsymbol{\Omega}} & -\boldsymbol{A}_{\boldsymbol{\Gamma}_{t}} \\
\boldsymbol{A}_{\boldsymbol{\Omega}}^{\mathrm{T}} & \mathbf{0} & \mathbf{0} \\
-\boldsymbol{A}_{\boldsymbol{\Gamma}_{t}}^{\mathrm{T}} & \mathbf{0} & \mathbf{0}
\end{array}\right]\left\{\begin{array}{l}
\boldsymbol{s}_{\Omega} \\
\boldsymbol{q}_{\Omega} \\
\boldsymbol{q}_{\boldsymbol{\Gamma}_{t}}
\end{array}\right\}=\left\{\begin{array}{c}
\boldsymbol{e}_{\boldsymbol{\Gamma}_{u}} \\
-\boldsymbol{Q}_{\Omega} \\
-\boldsymbol{Q}_{\boldsymbol{\Gamma}_{t}}
\end{array}\right\}} \\
& {\left[\begin{array}{cc}
\boldsymbol{F} & \boldsymbol{A} \\
\boldsymbol{A}^{T} & \mathbf{0}
\end{array}\right]\left\{\begin{array}{c}
\widetilde{\boldsymbol{\sigma}} \\
\widetilde{\boldsymbol{u}}
\end{array}\right\}=\left\{\begin{array}{c}
\boldsymbol{e}_{\boldsymbol{\Gamma}_{u}} \\
-\boldsymbol{Q}
\end{array}\right\}}
\end{aligned}
$$

Utilizando as matrizes diagonais dadas por:

$$
B=[\operatorname{diag}(F)]^{-\frac{1}{2}}
$$

os sistemas representados pelas eq.(B.1) e eq.(B.2) podem ser balanceados conforme a seguir:

$$
\begin{aligned}
& \bar{A} x=\bar{b} \\
& \therefore \bar{A}=\left[\begin{array}{ccc}
B F B & B A_{\Omega} & -B A_{\Gamma_{t}} \\
A_{\Omega}^{\mathrm{T}} \mathrm{B} & 0 & 0 \\
-A_{\Gamma_{t}}^{\mathrm{T}} \mathrm{B} & 0 & 0
\end{array}\right], x=\left\{\begin{array}{c}
B s_{\Omega} \\
\boldsymbol{q}_{\Omega} \\
\boldsymbol{q}_{\Gamma_{t}}
\end{array}\right\}, \bar{b}=\left\{\begin{array}{c}
\boldsymbol{e}_{\Gamma_{u}} \\
-Q_{\Omega} \\
-\boldsymbol{Q}_{\Gamma_{t}}
\end{array}\right\}
\end{aligned}
$$


$\overline{\boldsymbol{A}} \boldsymbol{x}=\overline{\boldsymbol{b}}$

$\therefore \bar{A}=\left[\begin{array}{cc}B \boldsymbol{F} B & \boldsymbol{B} \boldsymbol{A} \\ \boldsymbol{A}^{\mathrm{T}} \boldsymbol{B} & 0\end{array}\right], \boldsymbol{x}=\left\{\begin{array}{c}\boldsymbol{B} \widetilde{\boldsymbol{\sigma}} \\ \widetilde{\mathbf{u}}\end{array}\right\}, \bar{b}=\left\{\begin{array}{c}\boldsymbol{e}_{\boldsymbol{\Gamma}_{u}} \\ -\boldsymbol{Q}\end{array}\right\}$

Observando-se que todos os sistemas assumem a forma genérica $\overline{\boldsymbol{A}} \boldsymbol{x}=\overline{\boldsymbol{b}}$ e que $\operatorname{diag}(\boldsymbol{B F B})=\boldsymbol{I}$, efetua-se a seguinte operação:

$\widetilde{\boldsymbol{A}}=\overline{\boldsymbol{A}}+\delta \boldsymbol{I}$

sendo $0<\delta \ll 1$. Esta operação faz com que a matriz perturbada $\widetilde{\boldsymbol{A}}$ seja positiva definida e, portanto, inversível.

Assim, o algoritmo solução proposto por Strouboulis et al. (2000) é sucintamente definido a seguir:

i. $j=0 \quad \bar{x}_{0}=\mathbf{0} \quad \overline{\boldsymbol{r}}_{\mathbf{0}}=\overline{\boldsymbol{b}}$

ii. $\quad$ Resolver $\widetilde{A} a_{j}=\overline{\boldsymbol{r}}_{\boldsymbol{j}}$

iii. $\bar{x}_{j+1}=\bar{x}_{j}+a_{j}$

iv. $\quad \overline{\boldsymbol{r}}_{j+1}=\overline{\boldsymbol{r}}_{j}-\overline{\boldsymbol{A}}_{j} \boldsymbol{a}_{j}$

v. $j \leftarrow j+1$

vi. $\|R\|=\sqrt{\frac{\overline{\boldsymbol{r}}_{\boldsymbol{j}}^{\mathbf{T}} \overline{\boldsymbol{r}}_{\boldsymbol{j}}}{\overline{\overline{\boldsymbol{x}}_{\boldsymbol{j}}^{\mathbf{T}} \overline{\boldsymbol{A}} \boldsymbol{x}_{\boldsymbol{j}}}}}$

vii. Se $\|R\|>\epsilon$, voltar para o passo (ii).

onde $\boldsymbol{a}_{j}$ e $\overline{\boldsymbol{r}}_{\boldsymbol{j}}$ são o erro e o resíduo na iteração $j$, respectivamente. 